University of Tennessee Health Science Center

UTHSC Digital Commons

\title{
Macromolecular Complexes of Cystic Fibrosis Transmembrane Conductance Regulator Alter Fluid Transport in inflammatory Bowel Disorders
}

\author{
Kavisha Arora \\ University of Tennessee Health Science Center
}

Follow this and additional works at: https://dc.uthsc.edu/dissertations

Part of the Digestive System Diseases Commons, Medical Biochemistry Commons, and the Medical Cell Biology Commons

\section{Recommended Citation}

Arora, Kavisha, "Macromolecular Complexes of Cystic Fibrosis Transmembrane Conductance Regulator Alter Fluid Transport in inflammatory Bowel Disorders" (2013). Theses and Dissertations (ETD). Paper 19. http://dx.doi.org/10.21007/etd.cghs.2013.0018. 


\title{
Macromolecular Complexes of Cystic Fibrosis Transmembrane Conductance Regulator Alter Fluid Transport in inflammatory Bowel Disorders
}

\author{
Abstract \\ Macromolecular complexes of cystic fibrosis transmembrane conductance regulator (CFTR) comprise of \\ network of proteins that can regulate CAMP-/cGMP-activated CFTR chloride channel function. We report \\ the physical and functional coupling of CFTR with nitric oxide (NO) producing enzyme-inducible nitric \\ oxide synthase (iNOS) at the apical plasma membrane in inflammatory bowel disease (IBD). The complex \\ formation requires the scaffolding protein $\mathrm{Na}+/ \mathrm{H}+$ exchanger regulatory factor 2 (NHERF2). We \\ demonstrate that iNOS is overexpressed at or near the apical plasma membrane of gut epithelial cells in \\ IBD and, through the stimulation of the NO-cGMP pathway, generates compartmentalized cGMP \\ underneath the plasma membrane. This action triggers the hyperactivation of CFTR chloride channels \\ and causes secretory diarrhea phenotype in IBD. Inhibition of CFTR and iNOS activity attenuates diarrhea \\ in dextran sodium sulfate (DSS) induced colitis. Dissolution of the macromolecular complex in Nherf2 -/- \\ mice renders them resistant to CFTR-mediated secretory diarrhea in murine colitis models compared to \\ Nherf2+/+. Our findings have important therapeutic implications in IBD and other forms of \\ gastrointestinal inflammations.

\section{Document Type} \\ Dissertation \\ Degree Name \\ Doctor of Philosophy (PhD)

\section{Program} \\ Biomedical Sciences \\ Research Advisor \\ Anjaparavanda P. Naren, Ph.D. \\ Keywords \\ CFTR Diarrhea Gastrointestinal inflammations Inflammation-associated diarrhea Macromolecular \\ complexes Protein-protein interactions

\section{Subject Categories} \\ Digestive System Diseases | Medical Biochemistry | Medical Cell Biology | Medicine and Health Sciences
}




\title{
Macromolecular Complexes of Cystic Fibrosis Transmembrane Conductance Regulator Alter Fluid Transport in Inflammatory Bowel Disorders
}

\author{
A Dissertation \\ Presented for \\ The Graduate Studies Council \\ The University of Tennessee \\ Health Science Center \\ In Partial Fulfillment \\ Of the Requirements for the Degree \\ Doctor of Philosophy \\ From The University of Tennessee
}

By

Kavisha Arora

August 2013 
Copyright (C) 2013 by Kavisha Arora.

All rights reserved. 


\section{DEDICATION}

This dissertation is dedicated to my parents, Smt. Sumitra Arora and Shri D.K. Arora for their unconditional love, care and constant encouragement to succeed. 


\section{ACKNOWLEDGEMENTS}

I am truly grateful to my mentor Dr. Anjaparavanda P. Naren for providing me this opportunity to perform doctoral research in his laboratory. The graduate training in Dr. Naren's lab was an immense learning experience. Dr. Naren is a wonderful mentor and person and I am thankful to him for the opportunities, motivation, guidance and support he gave to me during the research period. I am sure this experience would help me succeed in my future endeavors as well.

I would like to express a sincere thanks to my dissertation committee members, Drs. Ivan C. Gerling, David R. Nelson, Randal K. Buddington, and Kaushik Parthasarathi for their support, valuable suggestions and direction to conduct my doctoral research and bring it to a completion.

Support from my lab members has been invaluable and critical to this research. I would like to thank Drs. Weiqiang Zhang, Aixia Ren, Chang Suk Moon, Himabindu Penmatsa, and Sunitha and Chandrima for making my work period both enjoyable and fruitful.

I would like to thank Department of Physiology, UTHSC for their administrative support and providing me J. Paul Quigley award and Gerwin fellowship awards.

In the end, I express my sincere gratitude to my parents for being exceptionally loving, supportive and caring, my husband Vineet Chawla for his love and encouragement and my siblings Sneha, Deepika and Anurag who have always been my strength. 


\begin{abstract}
Macromolecular complexes of cystic fibrosis transmembrane conductance regulator (CFTR) comprise of network of proteins that can regulate cAMP-/cGMPactivated CFTR chloride channel function. We report the physical and functional coupling of CFTR with nitric oxide (NO) producing enzyme-inducible nitric oxide synthase (iNOS) at the apical plasma membrane in inflammatory bowel disease (IBD). The complex formation requires the scaffolding protein $\mathrm{Na}^{+} / \mathrm{H}^{+}$exchanger regulatory factor 2 (NHERF2). We demonstrate that iNOS is overexpressed at or near the apical plasma membrane of gut epithelial cells in IBD and, through the stimulation of the NOcGMP pathway, generates compartmentalized cGMP underneath the plasma membrane. This action triggers the hyperactivation of CFTR chloride channels and causes secretory diarrhea phenotype in IBD. Inhibition of CFTR and iNOS activity attenuates diarrhea in dextran sodium sulfate (DSS) induced colitis. Dissolution of the macromolecular complex in Nherf $2^{-/}$mice renders them resistant to CFTR-mediated secretory diarrhea in murine colitis models compared to $N h e r f 2^{+/+}$. Our findings have important therapeutic implications in IBD and other forms of gastrointestinal inflammations.
\end{abstract}




\section{TABLE OF CONTENTS}

\section{CHAPTER 1. COMPARTMENTALIZED CYCLIC NUCLEOTIDE

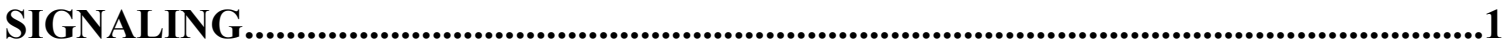

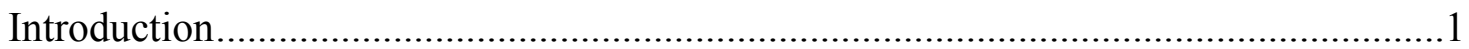

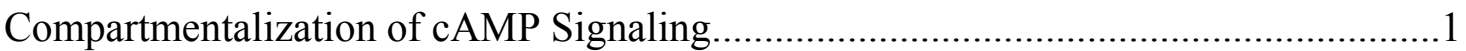
Adenylyl cyclases and G protein coupled receptors initiate cAMP compartments.....1 Phosphodiesterases and A-kinase anchoring proteins ...............................................2 Multiple drug resistance proteins: cyclic nucleotide efflux transporters .......................

Compartmentalization of cGMP Signaling .................................................................

\section{CHAPTER 2. MACROMOLECULAR COMPLEX OF CFTR AND} INDUCIBLE-NOS AT THE PLASMA MEMBRANE IN INFLAMMATORY BOWEL DISEASES .........................................................................................................6

Cystic Fibrosis Transmembrane Conductance Regulator...............................................6

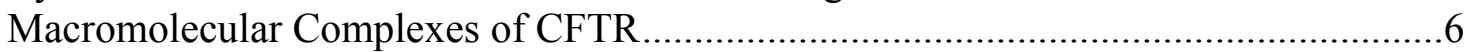

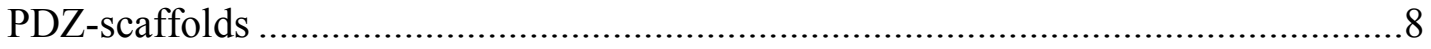

CFTR macromolecular complexes on PDZ-scaffolds ...............................................

CFTR is coupled to $\beta_{2}$ adrenergic receptor signaling via the formation of a NHERF1-mediated macromolecular complex with $\beta_{2}$ adrenergic receptor at the plasma membrane ...................................................................................... 9

A macromolecular complex of CFTR-NHERF1-NHE3 exists on the luminal surface of pancreatic duct ................................................................................. 9

CFTR is engaged in an inter-domain interaction with $\mathrm{Cl}^{-} / \mathrm{HCO}_{3}^{-}$exchangers on

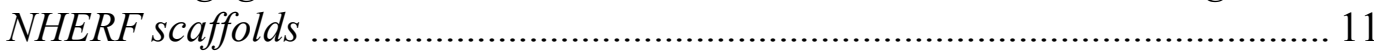
An LPA 2 -NHERF2-CFTR macromolecular signaling complex downregulates CFTR function in response to LPA .................................................................. 11 Spatiotemporal coupling of CFTR and MRP4 at the apical plasma membrane .. 12 CFTR functions as a conductance regulator by coupling physically and functionally with renal K channel..................................................................... 12 A macromolecular signaling complex of AQP9, NHERF1, and CFTR …............ 13

Inducible Nitric Oxide Synthase .............................................................................13

Compartmentalized NO Signaling ............................................................................15

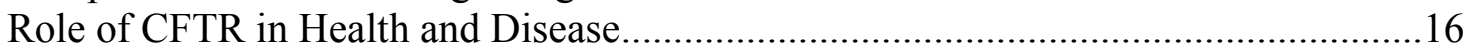

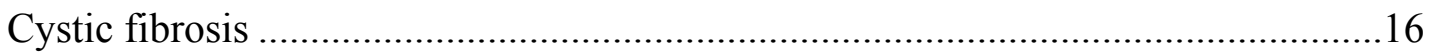

Role of CFTR in fluid secretion and diarrhea......................................................17

Inflammatory Bowel Disease..................................................................................20

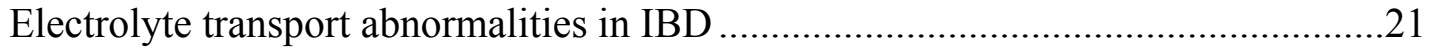

iNOS in inflammatory bowel disease ..............................................................21

Animal models of inflammatory bowel disorders ..................................................22

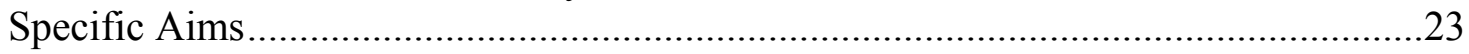

CHAPTER 3. METHODS …......................................................................................25

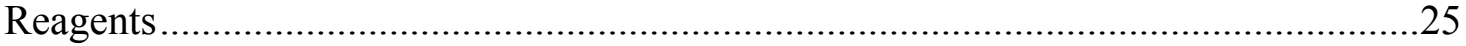

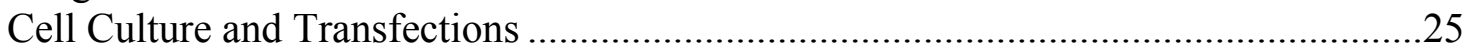


Purification of Flag- and HA-iNOS, and GST-His-S-and His-S-iNOS-C-tail

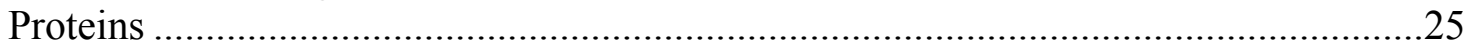

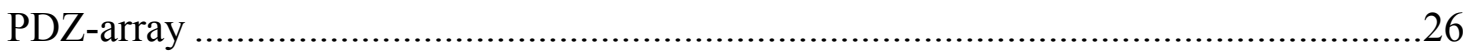

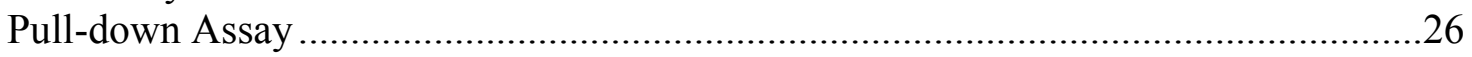

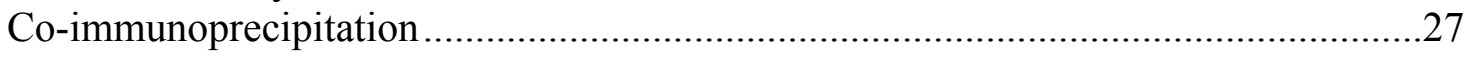

Direct Sensitized Fluorescence Resonance Energy Transfer Microscopy and Data

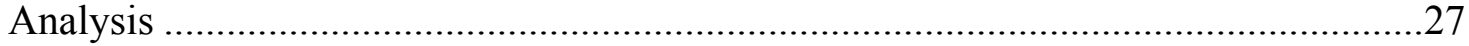

AlphaScreen $^{\mathrm{TM}}$ Assay for iNOS-NHERF2 Interaction.........................................27

Macromolecular Complex Assembly .....................................................................28

DSS-induced Colitis Murine Model ..........................................................................28

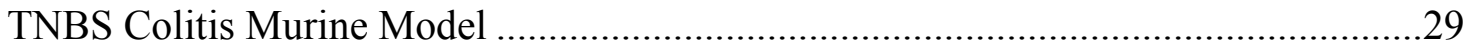

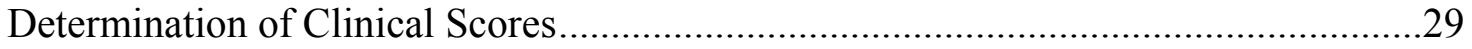

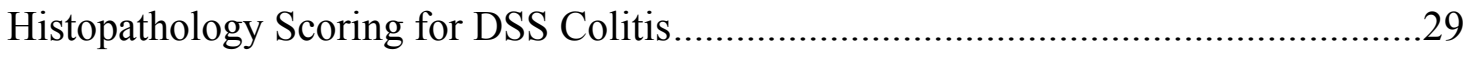

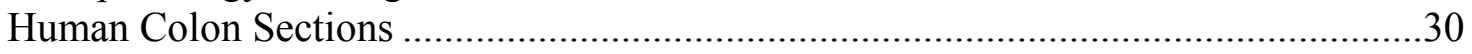

Human mRNA Expression Data Set....................................................................... 30

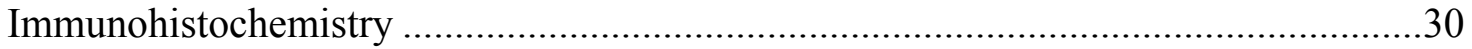

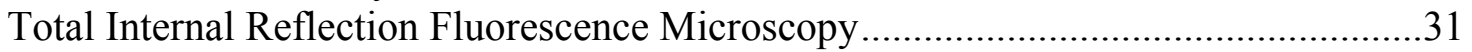

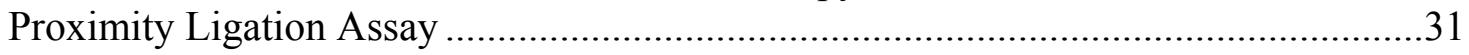

Measurement of CFTR-mediated I- Influx Using a YFP-based Halide Sensor..............31

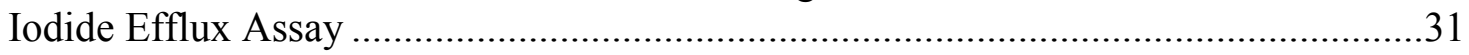

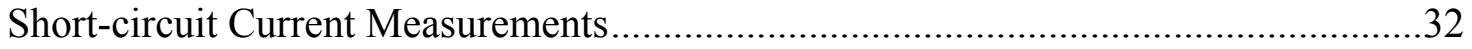

Ratiometric Fluorescence Resonance Energy Transfer Microscopy and Data

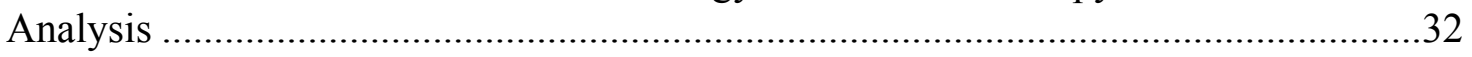

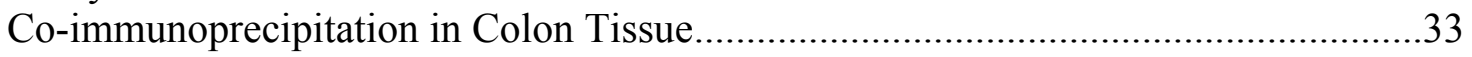

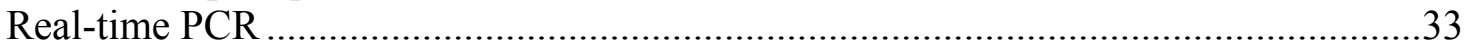

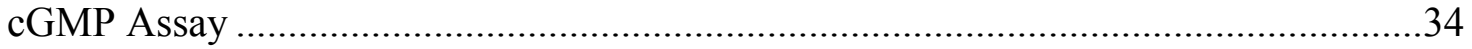

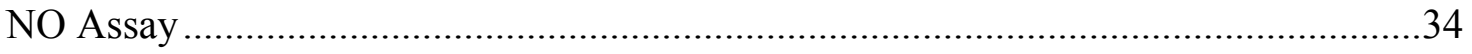

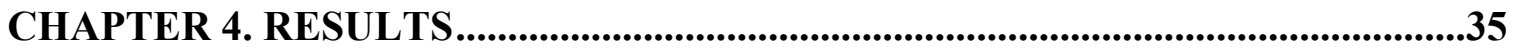

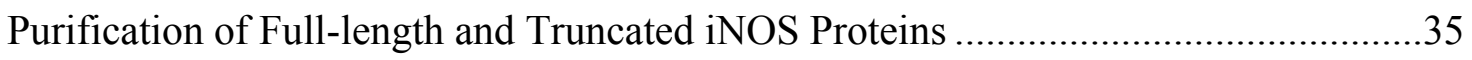

iNOS Exhibits High Affinity Interaction with NHERF2 ............................................

NHERF2 Bridges CFTR and iNOS into a Macromolecular Signaling Complex..........44

iNOS Protein Is Organized into Clusters at or near the Apical Plasma Membrane in

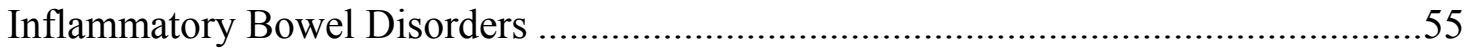

iNOS Potentially Constitutes cGMP Microdomains ..............................................64

iNOS Physical Interaction with CFTR Forms the Basis of CFTR Channel Activity

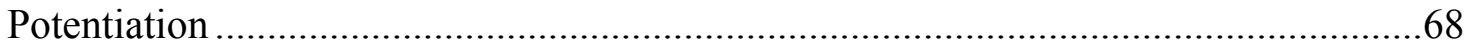

iNOS Potentiates CFTR Chloride Channel Function in a Cell Inflammatory Model ...73

Inhibition of CFTR and iNOS Activity Attenuates Diarrhea in DSS-induced Colitis ..79

Diarrhea Is Attenuated in Nherf2 $2^{-/}$Mice in DSS- and TNBS-induced Colitis..............87

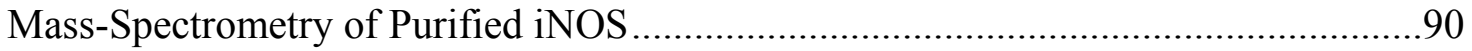

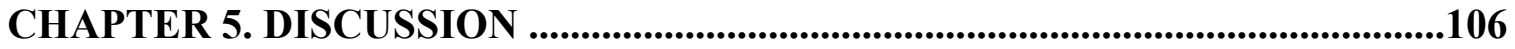

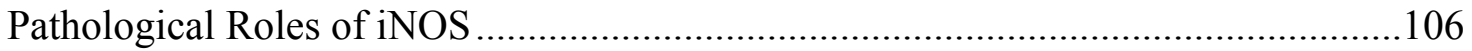

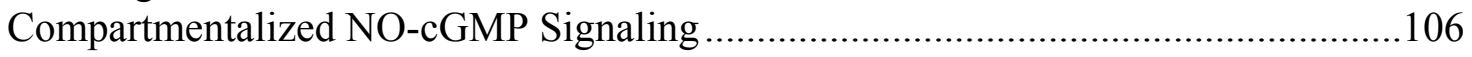

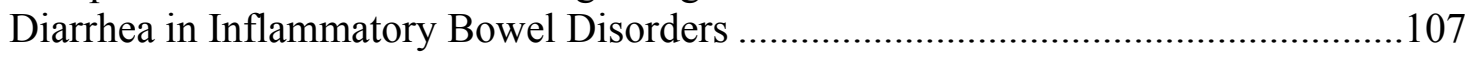


PDZ-scaffolds in the Spatiotemporal Regulation of CFTR .....................................109

CFTR within the NO-governed Compartments ...................................................... 110

Dissolution of Protein-protein Complexes as a Potential Therapy for IBD ................111

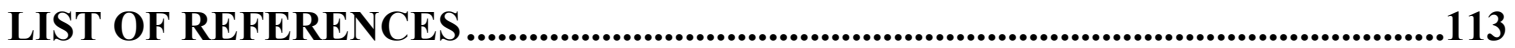

VITA 


\section{LIST OF TABLES}

Table 4-1. Mass-spectrometric analysis of immunopurified iNOS (Band 1)...............99

Table 4-2. Mass-spectrometric analysis of immunopurified iNOS (Band 2)..............100

Table 4-3. Mass-spectrometric analysis of immunopurified iNOS (Band 3)..............102

Table 4-4. $\quad$ Mass-spectrometric analysis of immunopurified iNOS (Band 4)...............103

Table 4-5. Mass-spectrometric analysis of immunopurified iNOS (Band 5)..............104

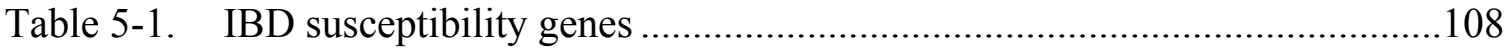




\section{LIST OF FIGURES}

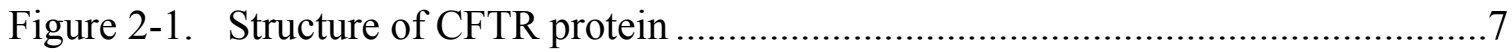

Figure 2-2. CFTR and PDZ protein binding partners.....................................................10

Figure 2-3. Representation of structural components of iNOS protein .............................14

Figure 2-4. Fluid transport model in a polarized intestinal epithelial cell.......................18

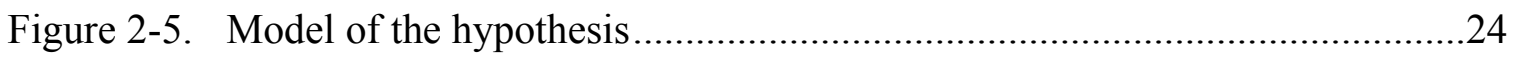

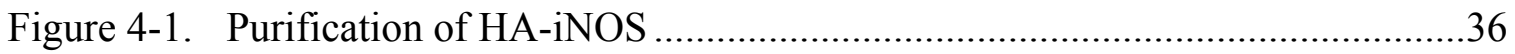

Figure 4-2. Purification of truncated iNOS proteins .........................................................37

Figure 4-3. iNOS preferentially binds NHERF2 among NHERF proteins .......................39

Figure 4-4. NHERF2 immunoprecipitates full-length iNOS from the cells.....................40

Figure 4-5. iNOS and NHERF2 exist within a protein complex in the cells...................42

Figure 4-6. iNOS and NHERF2 protein complex exists at the plasma membrane in

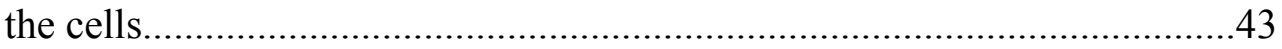

Figure 4-7. GST-NHERF2 and iNOS protein fragments interaction using AlphaScreen ${ }^{\mathrm{TM}}$ assay

Figure 4-8. AlphaScreen ${ }^{\mathrm{TM}}$ competition assay for GST-NHERF2 and iNOS-C-tail

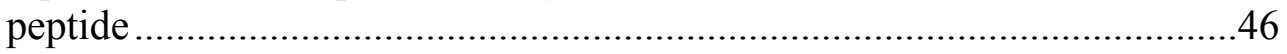

Figure 4-9. NHERF2 knockdown in HEK-293 cells....................................................

Figure 4-10. Role of NHERF2 in determining iNOS localization inside the cell .............48

Figure 4-11. PDZ-interaction based iNOS-NHERF2 coupling ..........................................49

Figure 4-12. PDZ-interaction dependence of iNOS localization in colon epithelial cells .50

Figure 4-13. CFTR-NHERF2-iNOS macromolecular complex

Figure 4-14. Knockdown of NHERF2 expression compromises CFTR-iNOS physical coupling at the plasma membrane

Figure 4-15. Modulating iNOS-CFTR interaction at the plasma membrane .54 
Figure 4-16. iNOS, CFTR and NHERF2 expression profiles in UC-affected human colon tissue...

Figure 4-17. H\&E stained normal and UC-affected human colon tissue sections

Figure 4-18. Immunostaining for iNOS, CFTR and NHERF2 expression in normal and UC-affected human colon sections.

Figure 4-19. Quantitated iNOS, CFTR and NHERF2 expression in normal and UCaffected human colon sections

Figure 4-20. iNOS clusters at the plasma membrane in UC-affected human colon epithelia

Figure 4-21. DSS-colitis in Nherf2 ${ }^{+/+}$and $\mathrm{Nherf}^{-/}$mice.............................................62

Figure 4-22. iNOS expression in DSS-colitis...............................................................63

Figure 4-23. iNOS-NHERF2 interaction in UC-affected human colon tissue ...................65

Figure 4-24. Pull-down of CFTR and iNOS using purified GST-NHERF2 in DSS-

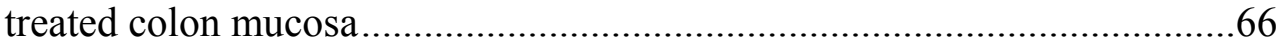

Figure 4-25. Immunoprecipitation of CFTR-NHERF2-iNOS macromolecular complex in DSS-treated colon tissue

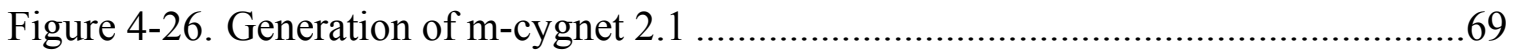

Figure 4-27. iNOS potentially constitutes cGMP microdomains at the plasma membrane

Figure 4-28. PDE5 expression in various cell systems...................................................71

Figure 4-29. CFTR expression in colon mucosal epithelial cells ......................................72

Figure 4-30. Knockdown of NHERF2 expression compromises CFTR-iNOS functional coupling .....................................................................................74

Figure 4-31. Zaprinast induced CFTR-chloride channel function......................................75

Figure 4-32. Localization of CFTR and iNOS in polarized HT29-CL19A cells ..............76

Figure 4-33. iNOS, CFTR and NHERF2 expression in cytomix challenged colon epithelial cells.

Figure 4-34. CFTR chloride channel function in cytomix-treated colon epithelial cells ..78

Figure 4-35. Inhibition of CFTR function causes partial protection against weight loss in DSS-colitis 
Figure 4-36. Inhibition of CFTR function attenuates fluid secretion in DSS-colitis.........81

Figure 4-37. Inhibition of CFTR function causes partial protection in DSS-colitis..........82

Figure 4-38. Histological scores are unaltered in the presence of CFTR inhibitor in DSS-colitis

Figure 4-39. Inhibition of iNOS function causes partial protection against weight loss and attenuates fluid secretion in DSS-colitis

Figure 4-40. Inhibition of iNOS function causes partial protection against DSS-colitis ..86

Figure 4-41. Inhibition of iNOS function improves histological scores in DSS-colitis....88

Figure 4-42. Inhibition of iNOS function affects NO-cGMP signaling in DSS-colitis.....89

Figure 4-43. Nherf $^{-/}$mice are partially protected against weight loss in DSS-colitis .....91

Figure 4-44. Nherf $2^{-/-}$mice exhibit reduced fluid secretion than $N h e r f 2^{+/+}$mice in DSS-colitis

Figure 4-45. Nherf $^{-/-}$mice are partially protected against diarrhea in DSS-colitis

Figure 4-46. Nherf $^{-/-}$mice are partially protected against weight loss in TNBS-colitis ..94

Figure 4-47. $N$ herf $2^{-/-}$mice exhibit reduced fluid secretion than $N h e r f 2^{+/+}$mice in TNBS-colitis as depicted in the exteriorized colon sections .95

Figure 4-48. Nherf $^{-/-}$mice are protected against diarrhea in TNBS-colitis .96

Figure 4-49. Histological scores are not different in $\mathrm{Nherf2}^{-/-}$mice than $N$ herf $2^{+/+}$ mice in TNBS-colitis

Figure 4-50. Immunoprecipitation of full-length HA-iNOS for mass-spectrometric analysis of bands $1-5$ as marked. .98 


\section{LIST OF ABBREVIATIONS}

$\beta_{1} / \beta_{2}$-AR

$\beta-\mathrm{ME}$

$\mu$

$\mathrm{ABC}$

AC

ADO

AKAP

AMP

AR

ATP

BSA

C

$\mathrm{Ca}^{2+}$

CAL

$\mathrm{CaM}$

cAMP

CAP70

CD

CF

CFP

CFTR

CFTR $_{\text {inh }}-172$

cGK

cGMP

$\mathrm{Cl}^{-}$

COPD

CTX

DLG5

DSP $\beta_{1} / \beta_{2}$-adrenergic receptor

$\beta$-mercaptoethanol

micro

ATP-binding cassette

adenylyl cyclase

adenosine

A-kinase-anchoring protein

adenosine monophosphate

adrenergic receptor

adenosine triphosphate

bovine serum albumin

carboxyl end

calcium

CFTR-associated ligand

calcium modulated protein

adenosine-3',5' -cyclicmonophosphate

CFTR-associated protein-70kDa

Crohn's disease

cystic fibrosis

cyan fluorescent protein

cystic fibrosis transmembrane conductance regulator

CFTR inhibitor-172

cGMP-dependent protein kinase

guanosine-3',5'-cyclicmonophosphate

chloride ion

chronic obstructive pulmonary disease

cholera toxin

disks large homolog 5

dithiobis succinimidyl propionate 
DSS

DTT

EDTA

EM-CCD

eNOS

EPAC

ER

FAD

FMN

FRET

Fsk

GAP

GI

GKAP

GPCR

GST

HA

$\mathrm{H}_{4} \mathrm{~B}$

HBSS

$\mathrm{HCl}$

HEK-293

HEPES

His

HRP

IBD

IFN- $\gamma$

$\mathrm{IgG}$

IKEPP

IL-1 $\beta$

Inh

iNOS dextran sodium sulphate

dithiothreitol

ethylene diamine tetraacetic acid

electron multiplying charge coupled device

endothelial nitric oxide synthase

exchange protein directly activated by cAMP

endoplasmic reticulum

flavin adenine dinucleotide

flavin mononucleotide

fluorescence resonance energy transfer

forskolin

GTPase-activating protein

gastrointestinal

G kinase anchoring protein

G-protein coupled receptor

glutathione-S-transferase

hemagglutinin

tetrahydrobiopterin

Hank's buffered salt solution

hydrochloric acid

human embryonic kidney-293

4-(2-hydroxyethyl)-1-piperazine ethanesulfonic acid

histidine

horse-radish peroxidase

inflammatory bowel diseases

interferon- $\gamma$

immunoglobulin $\mathrm{G}$

intestinal and kidney epithelial PDZ domain protein

interleukin-1 $\beta$

inhibitor

inducible nitric oxide synthase 


\begin{tabular}{|c|c|}
\hline IP & immunoprecipitation \\
\hline IPTG & isopropyl- $\beta$-D-1 thiogalactopyranoside \\
\hline $\mathrm{I}_{\mathrm{SC}}$ & short-circuit current \\
\hline $\mathrm{KCl}$ & potassium chloride \\
\hline $\mathrm{K}_{2} \mathrm{HPO}_{4}$ & dipotassium hydrogen phosphate \\
\hline $\mathrm{KH}_{2} \mathrm{PO}_{4}$ & monopotassium hydrogen phosphate \\
\hline LIC & ligation independent cloning \\
\hline L-NIL & N6-(1-iminoethyl)-L-lysine dihydrochloride \\
\hline $\mathrm{LPA}_{2}$ & lysophosphatidic acid receptor-2 \\
\hline LPS & lipopolysaccharide \\
\hline M & molar \\
\hline mAKAP & Muscle AKAP \\
\hline $\mathrm{mg}$ & milligram \\
\hline $\mathrm{MgCl}_{2}$ & magnesium chloride \\
\hline $\min$ & minute \\
\hline $\mathrm{ml}$ & milliliter \\
\hline $\mathrm{mm}$ & millimeter \\
\hline $\mathrm{mM}$ & millimolar \\
\hline MMC & macromolecular complex \\
\hline MRP & multidrug resistance protein \\
\hline MSD & membrane spanning domain \\
\hline $\mathrm{mV}$ & millivolts \\
\hline $\mathrm{N}$ & amino terminal end \\
\hline $\mathrm{Na}^{+}$ & sodium ion \\
\hline $\mathrm{NaCl}$ & sodium chloride \\
\hline $\mathrm{NADP}^{+}$ & nicotinamide adenine dinucleotide phosphate \\
\hline $\mathrm{NaHCO}_{3}$ & sodium bicarbonate \\
\hline $\mathrm{NaI}$ & sodium iodide \\
\hline $\mathrm{NaNO}_{3}$ & sodium nitrate \\
\hline NBD & nucleotide binding domain \\
\hline N-FRETc & normalized corrected FRET \\
\hline
\end{tabular}


NHE3

NHERF

NKCC

$\mathrm{nM}$

NMDA

NO

$\mathrm{P}$

PAGE

PBS

PDE

PDZ

PDZK1

pGC

PKA

PKC

PLA

PMSF

PSD95

PVDF

$\mathrm{R}$

ROMK

SDS

SEM

sGC

SHANK2

SNARE

TBS

TCA

TIRF

TNBS
$\mathrm{Na}^{+} / \mathrm{H}^{+}$exchanger 3

$\mathrm{Na}^{+} / \mathrm{H}^{+}$exchange regulatory factor

$\mathrm{Na}-\mathrm{K}-\mathrm{Cl}$ cotransporter

nanomolar

N-methyl-D-aspartate

nitric oxide

probability

polyacrylamide gel electrophoresis

phosphate buffered saline

phosphodiesterase

PSD95/Dlg5/ZO-1

PDZ domain-containing1

particulate guanylate cyclase

protein kinase $\mathrm{A}$

protein kinase $\mathrm{C}$

proximity ligation assay

phenyl methyl sulfonyl fluoride

post synaptic density protein 95

polyvinylidene difluoride

regulatory

renal outer medullary potassium channel

sodium dodecyl sulfate

standard error of the mean

soluble guanylate cyclase

SH3 and multiple ankyrin repeat domains protein 2

soluble N-ethylmaleimide-sensitive-factor attachment

protein receptor

Tris-base saline

trichloroacetic acid

total internal reflection fluorescence microscopy

2,4,6-Trinitrobenzenesulfonic acid 
TNF- $\alpha$

UC

WT

YFP

ZO-1 tumor necrosis factor- $\alpha$

ulcerative colitis

wild-type

yellow fluorescent protein

zonula occludens-1 protein 


\section{CHAPTER 1. COMPARTMENTALIZED CYCLIC NUCLEOTIDE SIGNALING}

\section{Introduction}

Cyclic nucleotides are the cellular messengers that relay environmental cues to mobilize cellular responses. Important cyclic nucleotides inside the cell are cyclic adenosine-3',5'-monophosphate (cAMP) and cyclic guanosine-3',5'-monophosphate (cGMP) (1). These cellular messengers are now known to be involved in various cellular responses to hormones and other types of stimuli and thereby, control diverse functions (e.g., metabolism, cell proliferation, differentiation, migration and fluid secretion) (2). It was first noticed by Bruxton and Brunton in 1980s that there is differentiation of cAMPdependent signaling processes in the cardiac myocytes (2-4). This concept came to be formulated as compartmentalized signaling. At the molecular level, compartmentalized cellular signaling would mean local sinks of cellular messengers capable of regulating signaling events in a nodal manner inside the cell. A signaling process gets compartmentalized when there is a generation of cyclic-nucleotides in the vicinity of their effectors and the signal termination mechanisms are set in place to prevent the spatial spread of the signaling. The receptors include protein kinases, kinase anchoring proteins, guanine-nucleotide exchange factors and cyclic-nucleotide-gated channels (1). Termination of the signaling process usually involves cyclic-nucleotide catabolizing enzymes phosphodiesterases (PDEs) and in some cases, recently characterized cyclicnucleotide efflux transporters $(2,5)$. The typical advantage of the process of compartmentalization is the spatiotemporal regulation of cyclic-nucleotide responsive proteins in specialized microdomains allowing high velocity and specificity of the responses. Apparently, compartmentalized signaling came into existence as an evolutionary response to selection pressure as eukaryotic cells needed to respond rapidly to the changing conditions and segregate simultaneously running signaling processes.

\section{Compartmentalization of cAMP Signaling}

\section{Adenylyl cyclases and G protein coupled receptors initiate cAMP compartments}

Generation of cAMP from ATP is dependent upon G-protein-coupled-receptor (GPCR)-mediated stimulation of adenylyl cyclases (ACs) $(3,6)$. It has been observed that certain membrane-bound AC isoforms can be selectively delivered to membrane rafts (rafts are dynamic cholesterol and sphingolipids rich structures of plasma membrane that play an important role in segregating signaling cascades at the membrane) where they exhibit exquisite sensitivity for local sub-micromolar $\mathrm{Ca}^{2+}$ gradients (7). The soluble ACs also exhibit compartmentalized distribution in close proximity with cAMP-responsive proteins and have been found to closely communicate with localized bicarbonate gradients (8). There are also reports on AC6 communicating with GPCRs $\beta_{1} / \beta_{2}-$ Adrenergic receptors (ARs) in caveolin-rich domains of plasma membrane with the 
coupling efficiency varying as a function of external stimulus (9). The selective patching of AC-GPCR signaling with an inherent role of heterogeneous physical barriers and with the contribution of cytoskeletal elements, provides the first level of cAMPcompartmentalization.

\section{Phosphodiesterases and A-kinase anchoring proteins}

In view of high diffusion rates of cAMP ( $220 \mu$ meters $\mathrm{s}-1)$, it is of utmost interest to know how cAMP mediated responses remain highly localized and specific ( 2 , 10). There are mechanisms to annul cAMP generated inside the cell and in fact, the cAMP hydrolysis has been estimated to be occurring faster than the cAMP synthesis (11). PDEs constitute the major force of catabolizing cyclic nucleotides. PDEs break the phosphodiester bond in cAMP and cGMP resulting in the formation of adenosine-5monophosphate (AMP) and guanosine-5-monophosphate (GMP), respectively. There are eleven mammalian PDE gene families, three cAMP-specific (PDE4, PDE7, and PDE8), three cGMP-specific (PDE5, PDE6, and PDE9), and five exhibit dual-specificities (PDE1, PDE2, PDE3, PDE10, and PDE11) (2). The diversity and specificities of PDEregulated signaling are derived from the variability of different isoforms in tissue-type expression, subcellular localization, and specific associations with adaptor and effector proteins (12). FRET-based sensors which fundamentally comprise of genetically modified cAMP-effectors such as EPAC and PKA subunits are immensely valuable for studying PDE-mediated compartmentalized signaling (13). Based on studies using these cyclic nucleotide sensors, it has been shown that pharmacological inhibition of PDEs does not alter the global levels of cyclic messengers but rather their sub-cellular distribution. It is now accepted that concerted actions of ACs and PDEs can create localized gradients of cAMP (3). This concept gets more sophisticated with an incurred process of GPCR desensitization because of the major regulatory role of PDE. For example, PDE4D5 can dampen $\beta_{2}$-AR signaling upon $\beta$-arrestin mediated recruitment of the enzyme to the phosphorylated $\beta_{2}$-AR. $\beta$-arrestins uncouple $\beta_{2}$-AR from Gs, essential for $\beta_{2}$-AR desensititzation (3). PDE4D5- $\beta$-arrestins complex will apparently accelerate the process of desensititzation where uncoupling of $\beta_{2}$-AR from Gs will occur concurrently with cAMP degradation by PDE4D5 and prepare $\beta_{2}$-AR for another round of agonist-induced activation (3). PDE3 inhibitor cilostazol can potentiate cystic fibrosis transmembrane conductance regulator (CFTR) $\mathrm{Cl}^{-}$channel function as PDE3A directly interacts with CFTR at the apical surface in epithelial cells (4). Notably, PDE3 inhibition generates compartmentalized cAMP at the plasma membrane causing highly localized activation of $\mathrm{CFTR}^{-}$channel function.

It has been found that PDEs can localize to specific subcellular domains via binding to A-Kinase anchoring protein (AKAP) (14). AKAP recognizes the $\mathrm{N}$-terminal of regulatory subunit II of cAMP-activated protein kinase A (PKA) and tethers PKA at distinct subcellular locations allowing spatiotemporal regulation of PKA substrates (3). Certain PDEs, AKAPs, kinases and phosphatases have analogous targeting mechanisms (specific for protein-protein or protein-lipid interactions) (3). PDE4D3 and PKA run a concerted negative feedback loop for cAMP signaling onto a muscle specific AKAP 
(mAKAP) maintained signaling module that controls the output of ventricular hypertrophy (15). Neuronal anchoring protein AKAP79 was found to bind to the phosphatase calcineurin and diminish the phosphorylation of PKA substrates and later, to protein kinase $\mathrm{C}$ (PKC) which can act on an overlapping but distinct set of substrates (16, 17). Also, AKAPs exhibit discrete subcellular targeting where they can nucleate distinct protein complexes. The plasma membrane-associated Yotiao, a smallest splice variant of the AKAP9 family tethers PKA, protein phosphatase 1, PDE4D3, the NR1 subunit of the NMDA receptor, IP3 receptor, and the $\mathrm{K}^{+}$channel subunit KCNQ1 to control $\mathrm{I}_{\mathrm{Ks}}$ currents in the heart (18). Ezrin is an AKAP enriched in microvilli on the apical side of polarized epithelial cells which regulates the PKA-mediated inhibition of $\mathrm{Na}^{+} / \mathrm{H}^{+}$exchanger 3 (NHE3) and activation of CFTR via Ezrin-binding protein $\mathrm{Na}^{+} / \mathrm{H}^{+}$exchange regulatory factor 1 (NHERF1) (19-22). Ezrin also binds F-actin which determines the stability of CFTR at the plasma membrane (23). Thus, AKAPs assemble large multi-protein complexes which act as nodes of enzymatic and regulatory activities.

\section{Multiple drug resistance proteins: cyclic nucleotide efflux transporters}

Davoren and Sutherland observed that hormonal stimulation of the cells would alter not only the intracellular but also the extracellular cAMP levels (2). To explain this observation, it was rationalized that cells use an unidentified cyclic nucleotide export system. Later, a member of the $\mathrm{ABC}$ transporter family termed as multidrug resistance protein 4 (MRP4; also known as ABCC4), was identified to efflux cAMP as well as $\operatorname{cGMP}(24,25)$. The affinity constant of MRP4 for cGMP $(10 \mu \mathrm{M})$ is lower than for cAMP $(45 \mu \mathrm{M})(25)$.

MRP4 is ubiquitously expressed in many cell types polarized and non-polarized and in the former type lines both apical and basolateral membranes of the epithelial cells (2). Interestingly, MRP4 inhibition alone could not substantially change the total cyclic nucleotide levels, which clearly signifies the role of MRP4 in compartmentalized signaling. Li et al. reported an important role of apical MRP4 in regulating CFTR function in a highly compartmentalized manner in the gut epithelial cells (5). Recent studies reported that MRP4 can be anchored by caveolins in the plasma membrane where it participates in highly segregated signaling modules (26). We recently demonstrated that MRP4 can control distinct PKA activities at the leading vs. lagging edge of a migrating fibroblast to regulate the migration process (27). Also, regulation of migration gets biphasic as MRP4 segregates between cAMP and cGMP-mediated signaling and directs different migration outputs (27). Other additional $\mathrm{ABC}$ transporters have been identified to export cyclic nucleotides which include MRP5, ABCG2 and MRP8 (2). Therefore, cyclic nucleotide extrusion from the cell by certain $\mathrm{ABC}$ transporters represents an additional mechanism to attenuate the signaling process and also, there have been observations fostering the fact that these two mechanisms may act compensatory or in concert (2). 


\section{Compartmentalization of cGMP Signaling}

Studies related to elucidating cGMP signaling are less well-understood compared to the cAMP pathways. Robert F. Furchgott, Louis J. Ignarro and Ferid Murad won 1998 Nobel Prize in Physiology or Medicine for studying the significance of nitric oxide (NO)cGMP pathway in cardiovascular health which underscores the importance of cGMP pathways in the living systems. cGMP plays role in several important biological functions such as vascular tone regulation, epithelial electrolyte transport, migration, neurotransmission and immunomodulation (28). cGMP is synthesized by NO-responsive soluble ( $\mathrm{sGC}$ ) or membrane-associated particulate ( $\mathrm{pGC}$ ) form of guanylyl cyclases. Seven isoforms of $\mathrm{pGCs}$ have been identified which include natriuretic peptide receptors GC-A and GC-B, GC-C which are responsive to bacterial heat-stable enterotoxins, guanylin, and uroguanylin and orphan receptors GC-D to G (28). The process of cGMP compartmentation is vaguely known compared to cAMP however; the same question can be applied asking how different extracellular cues fundamentally working through a common intracellular messenger can produce varied biological responses. It is likely, that similar mechanisms and components to enable compartmentalization of cGMP signaling would exist as are known for cAMP. Recent studies report that GC-C communicates with intracellular p21-activated kinase via Rac GTPase to regulate cell migration and actin cytoskeletal restructuring (29). The basis of this process would be asymmetric and segregated signaling that regulates the directed cell migration $(27,29)$. cGMP-dependent protein kinases (PKGs) are the most important effectors of cGMP response. G-kinase anchoring proteins (GKAPs) are the proteins required for the dissemination of PKGsignaling. However, the field is very new and limited information is available. Scaffolding protein NHERF2 has been shown to function as (GKAP) to mediate cGMPdependent regulation of NHE3 allowing the compartmentalized regulation of the protein (30). Since cGMP can increase CFTR chloride activity and it is known that CFTR can bind to NHERF2, NHERF2 can regulate cGMP-dependent activation of CFTR which would also likely to be occurring in membrane restricted compartments. Few other GKAPs include vimentin, guanylate cyclase A, troponin $\mathrm{T}$, myosin light chain kinase, testicular golgi protein GKAP42 (30). Neutralizing components of cGMP signaling importantly include cGMP catabolizing PDEs: PDE5, PDE6, and PDE9. Five PDEs (PDE1, PDE2, PDE3, PDE10, and PDE11) exhibit dual specificities for cAMP and cGMP. These dual-specificity PDEs (in particular reported to be PDE2 and PDE3) play critical roles in cross-talks between cGMP and cAMP-dependent signaling $(31,32)$. An important role of spatiotemporal regulation of functional NO-cGMP signaling system has been described in cell fate decisions and lineage determination during the early differentiation process of embryonic stem cells (33).

In this study we determine how compartmental cGMP becomes the pathophysiological basis of CFTR-dependent diarrhea in inflammatory bowel disorders (IBD). We elucidate formation of a CFTR-containing macromolecular complex as the molecular basis of diarrheal phenotype of IBD.

In conclusion, the concept of compartmentalized signaling as of today is astonishingly advanced and intricate. This is primarily attributed to appreciating how the 
process of compartmentalized signaling is fundamentally relevant to cellular behavior in health and disease and the availability of scientific tools which allow monitoring the process very efficiently. 


\section{CHAPTER 2. MACROMOLECULAR COMPLEX OF CFTR AND INDUCIBLE-NOS AT THE PLASMA MEMBRANE IN INFLAMMATORY BOWEL DISEASES}

\section{Cystic Fibrosis Transmembrane Conductance Regulator}

Compartmentalized signaling has been shown to extensively regulate the activity of $\mathrm{Cl}^{-}$channel CFTR (or ABCC7) at the plasma membrane $(34,35)$. CFTR is a 1480 amino-acid long membrane-bound protein belonging to the ATP-binding cassette transporter $(\mathrm{ABC})$ superfamily which consumes energy from ATP hydrolysis to transport a wide-variety of substrates $(34,35)$. CFTR consists of two homologous halves, each composed of a hydrophobic membrane-spanning domain (MSD) containing six transmembrane helices and a cytosolic nucleotide binding domain (NBD). The two NBDs are linked by a cytoplasmic regulatory domain ( $\mathrm{R}$ domain) which contains multiple consensus phosphorylation sites for several kinases. The phosphorylation state of CFTR determines the channel open probability (P). The structure of CFTR is shown in Figure 2-1.

Interestingly, it was reported that the amino $(\mathrm{N})$ terminus cytoplasmic region of CFTR is involved in a direct interaction with the $\mathrm{R}$ domain via a conserved set of residues which will determine the steady-state activity of CFTR (36). CFTR is an epithelial cell protein present on the apical membranes in the gut, lung airways, sweat gland, pancreas and urogenital tract that regulates fluid balance across the epithelia lining these organs (37).

\section{Macromolecular Complexes of CFTR}

CFTR is a cAMP-/cGMP-activated $\mathrm{Cl}^{-} / \mathrm{HCO}_{3}{ }^{-}$transporter $(38,39)$. The most interesting aspect of CFTR regulation is the formation of multi-protein complexes via its $\mathrm{N}$ and carboxyl (C) terminals. A direct interaction at the $\mathrm{N}$ terminus of CFTR with the regulatory SNARE ( soluble N-ethylmaleimide-sensitive-factor attachment protein receptor) protein Syntaxin 1A negatively modulates CFTR function and is subject to competitive inhibition by Syntaxin 1A-binding protein Munc 18 (40). Such interactions will be critical to regulate the dynamics of CFTR channel activity according to a physiological cue. Macromolecular complexes of CFTR involving regulators and effectors of cAMP would lead to highly compartmentalized regulation of CFTR within cAMP microdomains at the plasma membrane. The cAMP-elevating ligand adenosine (ADO; $1-20 \mu \mathrm{M}$ ) which acts through adenosine receptor subtypes belonging to GPCR family can also stimulate CFTR-mediated $\mathrm{Cl}^{-}$currents in a compartmentalized manner at the apical cell membrane of polarized epithelial cells (41). In cell-attached single-channel recordings of CFTR, it was observed that the AC stimulating agent forskolin (Fsk), or cell-permeant cpt-cAMP always elicited a global cAMP response and activated CFTR irrespective of whether these reagents were applied in the pipette or in the bath (42). In contrast sub-micromolar concentrations of ADO evoked a localized activation of CFTR, 


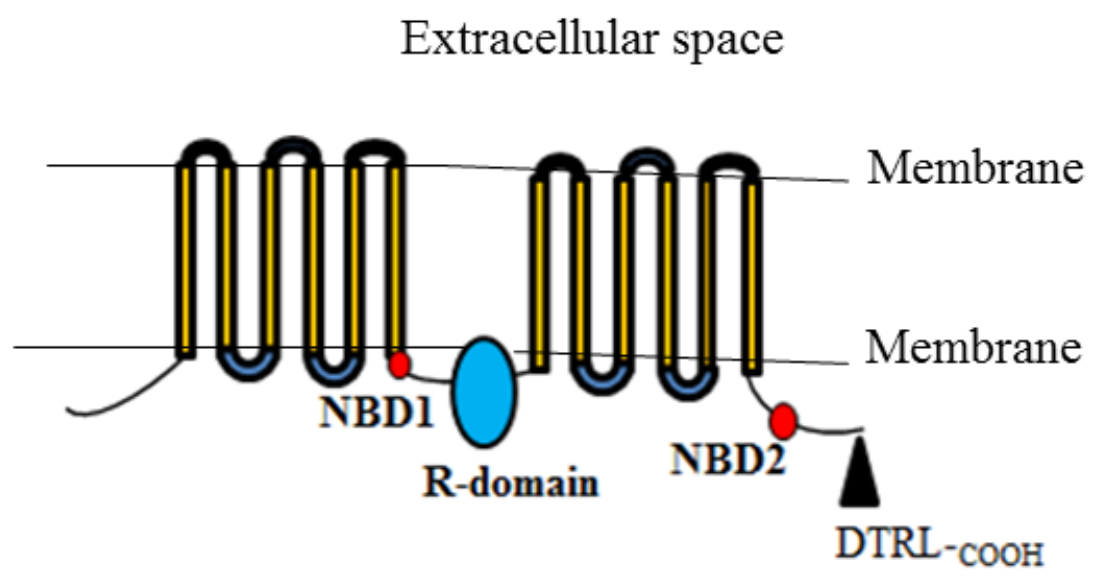

Cytosol

Figure 2-1. Structure of CFTR protein

CFTR protein topology showing various domains of the CFTR protein: two membrane spanning domains (colored in orange), two nucleotide binding domains (colored in red) and a regulatory domain (colored in light blue). 
as ADO when added to the bath failed to activate CFTR function (42).

\section{PDZ-scaffolds}

Most of the macromolecular formations of CFTR at the $\mathrm{C}$ terminus are mediated by a class of scaffolding proteins termed as PSD-95/DLG-5/ZO-1 (PDZ)-domain proteins $(34,37)$. PDZ domains are named after the first three identified PDZ-domain containing proteins which include postsynaptic density protein-95 (PSD-95), the drosophila junctional protein Disc-large (DLG), and the epithelial tight junction protein zonula occludens (ZO)-1. PDZ modules are small approximately 90 amino-acids long and recognize four to six residues of the target proteins. PDZ domains constitute among the most commonly expressed domains in an eukaryotic genome and have been estimated to number greater than 400 in human (43). With such an abundance and diversity, PDZproteins apparently regulate many signal transduction events inside the cell (44). The primary role of PDZ proteins is to function as scaffolds to assemble large protein complexes that facilitate coordination and cross-talk within the same or even distinct pathways.

Elucidation of the structures of a number of PDZ domains alone, or in the ligandbound state have provided valuable insights into how auxillary structural components of a globular protein in an interaction with an adaptor protein modulate the activity of the core domain (45). The canonical structural configuration of PDZ domains comprises $6 \beta$ strands $(\beta \mathrm{A} \sim \beta \mathrm{F})$, a short $\alpha$-helix $(\alpha \mathrm{A})$ and a long $\alpha$-helix $(\alpha \mathrm{B})$. Single hydrophobic cleft between the $\alpha \mathrm{B}$-helix and $\beta \mathrm{B}$-strand structures, with the highly conserved carboxylatebinding loop $(\mathrm{R} / \mathrm{K}-\mathrm{XXX}-\mathrm{G}-\Phi-\mathrm{G}-\Phi$ motif, where $\mathrm{X}$ is any amino acid residue and $\Phi$ is a hydrophobic residue) located before the $\beta B$ strand, forms the peptide-binding site (44). Several modes of regulation and deregulation of PDZ-dependent interactions have been identified which include phosphorylation of the PDZ-binding motif in the target proteins, formation of disulphide linkages with in the PDZ-proteins, occupancy of the peptidebinding cleft by an inbuilt PDZ-binding motif and allosteric inhibition (44).

With the ability of PDZ-proteins to regulate a huge web of signal transduction events, many of them have now been implicated in cancer and neurodegenerative disorders (46). This is based on the studies in animal models and using blocking peptides to competitively inhibit native interactions which identified that certain PDZ-proteins integrate disease-associated proteins. The obvious advantage of targeting scaffolding proteins is the simultaneous control of multiple signaling cascades in complex diseases by hitting a single protein rather than multiple disease-related proteins. Subtle-binding specificities of PDZ-domains have been moderately classified. Also, mapping of the PDZ domains and narrowing down to smaller binding segments would confer a high degree of specificity and flexibility in the use of such therapeutic approaches. The bigger challenge in the future in using PDZ-interaction modules as drug targets is the promising in vivo outcome and the ease of manipulation of this approach without any significant side effects. 


\section{CFTR macromolecular complexes on PDZ-scaffolds}

CFTR possesses a class I PDZ-domain binding motif DTRL_COOH at the C terminus that mediates the binding of CFTR to several PDZ-domain proteins, including $\mathrm{Na}^{+} / \mathrm{H}^{+}$exchange regulatory factor 1 (NHERF1), NHERF2, CFTR-associated protein 70 kDa (CAP70; also known as PDZK1), NHERF4 and CFTR-associated ligand (CAL) (47). Various PDZ-mediated interactions of CFTR and their implications are shown in Figure 2-2.

PDZ-proteins are usually bivalent or multivalent harboring multiple PDZdomains. This structural feature of PDZ-proteins is known to contribute to CFTRdimerization and most interestingly, constitution of large multiple protein complexes which act as hubs of enzymatic and regulatory activities $(48,49)$. Also, the crosslinking between two CFTR molecules or CFTR and other interacting proteins can be modulated by the stoichiometry of CFTR-PDZ-protein interaction (48). Few studies also suggested that CFTR dimerization is only cAMP-regulated and does not require NHERF proteins (50). Other roles of PDZ-mediated interactions of CFTR have been identified which include trafficking, polarized expression and membrane-associated mobility and stabilization of the protein (34).

CFTR is coupled to $\beta_{2}$ adrenergic receptor signaling via the formation of a NHERF1mediated macromolecular complex with $\beta_{2}$ adrenergic receptor at the plasma membrane

CFTR couples $\beta_{2}$-AR via NHERF1 at the apical surface of epithelial cells (22). NHERF1 recognizes Ezrin (an AKAP) via its ezrin/radixin/moesin (ERM) domain at the C-terminus that positions PKA close to CFTR. On agonist activation of the receptor, AC is stimulated generating highly compartmentalized cAMP which leads to phosphorylation-based activation of the channel (22). In this study they also reported that the macromolecular formation is dependent on the phosphorylation status of CFTR as it negatively regulates the binding of the CFTR to NHERF1 and also deletion of the R domain in CFTR cancelled dose-dependent inhibitory effect of PKA on complex formation. In addition, impaired $\beta_{2}$-AR-mediated regulation of CFTR function has also been recapitulated in NHERF1 deficient mice (49). Also, highly similar proteins NHERF2 and PDZK1 failed to compensate for $\beta_{2}$-AR-mediated response, indicating that complex-mediated regulation of CFTR is NHERF1 specific. It suggests that there is a high degree of specificity associated with a compartmentalized response. It is also predicted that such compartmentalization based signaling can get compromised in mutant and defective CFTR protein.

A macromolecular complex of CFTR-NHERF1-NHE3 exists on the luminal surface of pancreatic duct

An interesting component of CFTR function is regulation of luminal $\mathrm{HCO}_{3}{ }^{-}$levels. CFTR function increases $\mathrm{HCO}_{3}{ }^{-}$secretion when stimulated and reduces $\mathrm{HCO}_{3}{ }^{-}$salvaging 

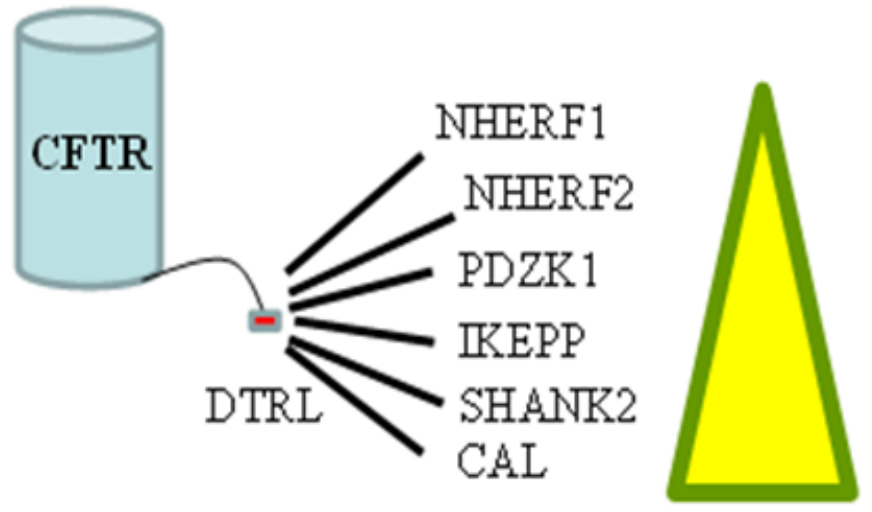

Dynamics of CFTR-

PDZ proteins

interaction is a

function of :

- Tissue-specific expression

- Sub-cellular location

- Physiological stimulus

Figure 2-2. CFTR and PDZ protein binding partners 
by other luminal transporters such as $\mathrm{Na}^{+} / \mathrm{H}^{+}$exchanger regulatory factor 3 (NHE3) in the resting state (51). Later, these two proteins were found to co-exist as a part of a macromolecular complex on the apical surface of pancreatic ductal cells. CFTR in this complex acutely mediates cAMP-monitored inhibition of NHE3 function and in a chronic mechanism elevate expression levels of NHE3 at the plasma membrane $(34,52,53)$. NHERF1 mediates the functional coupling between CFTR and NHE3 with the first PDZ domain of NHERF1 preferentially complexing with CFTR and NHE3 associating with the latter half including the second PDZ domain and the C-terminal ERM binding domain of NHERF1 (34). With NHERF1as an Ezrin binding protein, regulation of NHE3 by PKA would be instantaneous and highly compartmentalized. Interestingly, mutant CFTR failed to elicit inhibition of NHE3 function or increase in its expression level, implying that an intact CFTR is required for mediating this compartmentalized regulation. Also, the macromolecular association possibly has a significant role in stabilizing NHE3 at the plasma membrane. This concept runs parallel to the stabilization of CFTR at the plasma membrane by NHERF1-mediated coupling of the protein to actin cytoskeleton (54).

CFTR is engaged in an inter-domain interaction with $\mathrm{Cl}^{-} / \mathrm{HCO}_{3}{ }^{-}$exchangers on $\mathrm{NHERF}$ scaffolds

The mechanism where CFTR can stimulate $\mathrm{HCO}_{3}{ }^{-}$secretion in the activated state involves coupling of CFTR with $\mathrm{Cl}^{-} / \mathrm{HCO}_{3}{ }^{-}$exchangers which include members of the SLC26 exchanger family SLC26A3/DRA and SLC26A6/PAT1. SLC26 transporters and CFTR assemble together onto NHERF scaffolds $(55,56)$. Also, an interdomain interaction between conserved sulphate transporter and anti-sigma antagonist domain of SLC26 transporters at the C-terminal and the R domain of CFTR reciprocally modulates activities of these transporters (57). Consequently, stimulation of DRA could increase the open probability of CFTR by 6-fold and phosphorylation of the R domain of CFTR resulted in an increase in DRA activity. Importantly, NHERF1 and NHERF2 scaffolding dynamics within the complex would determine the functional output of this association (58). This inter-regulated assembly of SLC26 transporters and CFTR channel functions as a $\mathrm{HCO}^{-}$flux regulating system in the apical membranes of epithelial cells.

An LPA 2 -NHERF2-CFTR macromolecular signaling complex downregulates CFTR function in response to $L P A$

CFTR was also reported to associate with another GPCR lysophosphatidic acid receptor-2 $\left(\mathrm{LPA}_{2}\right)$ bridged by scaffolding protein NHERF2 (42). Supposedly, LPA 2 in this complex is coupled to Gai G-protein which inhibits AC function. LPA $A_{2}$ is one of the many receptors for lysophosphatidic acid, a physiological phospholipid present in the blood (59). Therefore upon stimulation of $\mathrm{LPA}_{2}$ with LPA binding, CFTR activity within the complex is downregulated because of the reduced flux of cAMP near CFTR. LPA 2 mediated downregulation of CFTR function conferred protection against cholera toxin (CTX)-induced secretory diarrhea in which CFTR has a predominant role (42). An interesting component of this study was LPA did not alter the global cAMP levels in the 
presence of low concentration ADO, implying that the LPA-mediated effect via $\mathrm{LPA}_{2}$ on CFTR channel function was highly compartmentalized. Functional consequences of LPA on CFTR activity also suggested that diet supplementation with LPA or consumption of an LPA-enriched diet can be considered as an alternative therapy for controlling watery diarrhea.

\section{Spatiotemporal coupling of CFTR and MRP4 at the apical plasma membrane}

Another evidence of compartmentalized regulation of CFTR is the association of CFTR with the cAMP/cGMP-efflux transporter multi-drug resistance protein 4 (MRP4) mediated by PDZK1 (5). Inhibition of MRP4 generates a localized pool of cAMP in the vicinity of CFTR allowing spatiotemporal regulation of CFTR channel function in certain plasma membrane microdomains. Low dose adenosine in the presence of MRP4 inhibitor MK571substantially potentiated CFTR-mediated $\mathrm{Cl}^{-}$currents. However, higher doses of ADO $(>20 \mu \mathrm{M})$ failed to significantly alter CFTR function in the presence of MK571. $\mathrm{Mrp}^{-/-}$and $\mathrm{Mrp}^{+/ /}$mice with inhibition of MRP4, showed ameliorated CTX-induced diarrheal phenotype. MRP4 lines apical and basolateral surfaces of various cell-types and its proximity to cyclic-nucleotide effectors would allow the instantaneous modulation of their activities as it regulates the flux of cyclic messengers without the need of degrading them.

Cyclic nucleotide extrusion by MRP4 represents a mean of maintaining cyclic nucleotide balance inside the cell other than degradation by PDEs.

CFTR functions as a conductance regulator by coupling physically and functionally with renal K channel

The role of CFTR as a conductance regulator other than a $\mathrm{Cl}^{-}$pump was validated in its ability to regulate the activity of several transporters in the epithelial membranes. A prominent example of such an ability of CFTR is the formation of a macromolecular complex of CFTR and renal secretory renal outer medullar potassium (ROMK) channel (also known as Kir 1.1 or KCNJ1) at the apical membrane (59). Interestingly, CFTR is expressed throughout the nephron. CFTR-ROMK complex would regulate salt absorption by controlling $\mathrm{K}^{+}$recycling/secretion across the thick ascending limb of Henle's loop, distal tubule, and collecting duct in the kidney.

ROMK binds to NHERF scaffolding proteins which is a strong determinant of plasmalemmal expression of active ROMK and also coordination of physical and functional coupling between CFTR and ROMK. Primarily, this association regulates channel sensitivity to sulfonylurea, glibenclamide and intracellular ATP. PKA activity acts as a functional switch that determines the CFTR-mediated regulatory effects on ROMK. 
CF patients have major fertility issues among both men and women. The major cause of CF-males being infertile is the anatomical absence of vas deferens and atrophied epididymis at certain regions and, possibly it is the presence of thick and tenacious cervical mucus which causes females to be reproductively compromised (60). It has been shown that CFTR is not only required to developmentally regulate reproductive systems but also for CFTR-mediated regulation of $\mathrm{HCO}_{3}{ }^{-}$secretion in the uterus which is critical for the fertilizing capacity of the sperm (60). Fluid content and composition of the male reproductive tract is critical to control sperm density and motility. CFTR has a prominent role in regulating luminal contents in distal epididymis and vas deferens. Recently, a NHERF1-mediated complex of CFTR with another apical protein aquaporin 9 (AQ9) was identified in the principal cells of the epididymis and the vas deferens (61). AQ9 belongs to a class of transporters which permeates bulk water and neutral solutes such as glycerol across the epithelia in response to an osmotic gradient $(62,63)$. AQ9 lines the apical membranes of both ciliated principle cells along the epididymis and vas deferens and non-ciliated cells of the efferent ducts fairly co-localizing with CFTR (34). Another study demonstrated that CFTR and AQ9 could synergistically modulate luminal fluid composition at these regions (64). Since AQ9 could co-immunoprecipate both phosphorylated and non-phosphorylated forms of NHERF1, the phosphorylation status of NHERF1 would act as a determinant of the regulatory influences of CFTR on AQ9 (61). This stems from the fact that phosphorylated NHERF1 causes CFTR to switch from its chloride channel activity to a conductance regulator. Similar aspects of regulation hold for the CFTR-ROMK protein complex (65).

These studies clearly suggest the potential of CFTR in engaging in multipleprotein containing signaling complexes which would ensure high fidelity and efficiency of CFTR regulation and may also have pathophysiological relevance.

\section{Inducible Nitric Oxide Synthase}

Inducible nitric oxide synthase (iNOS or NOS2) is a 1153 amino acids long protein which is non-constitutively expressed in many cell types and is usually upregulated in response to an inflammatory trigger (66). In contrast to other NOS forms which include endothelial NOS and neuronal NOS, iNOS does not get regulated by intracellular changes in $\mathrm{Ca}^{2+}$ (67). Several cell-types such as endothelial cells, hepatocytes, enterocytes, immune cells and smooth muscle cells can synthesize iNOS (68).

Transcriptional activation of iNOS is under the control of the NFאB pathway which gets stimulated in response to cytokines and lipopolysaccharides (LPS). iNOS is a modular protein with oxygenase and reductase domains at the $\mathrm{N}$-and $\mathrm{C}$-terminal halves, respectively. The oxygenase domain contains the binding site for heme, L-arginine and tetrahydrobiopterin $\left(\mathrm{BH}_{4}\right)$. The reductase domain harbors CaM, NADPH, FAD and FMN binding regions. The structure of full-length iNOS is represented in Figure 2-3. 


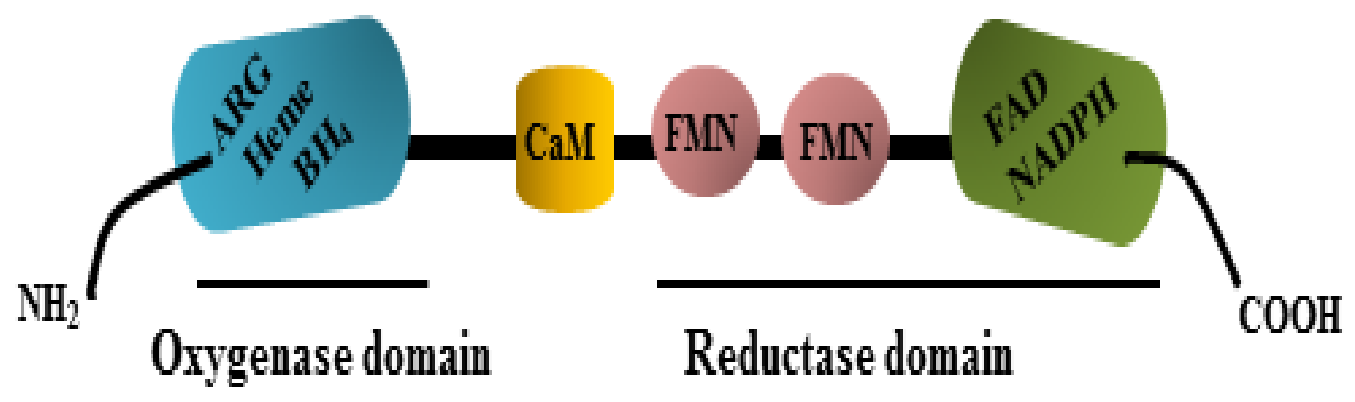

Figure 2-3. Representation of structural components of iNOS protein 
NOS synthesizes NO in a two- step oxidative conversion of L-arginine to NO and L-citrulline in the presence of cofactors including heme, FMN, FAD and $\mathrm{BH}_{4}$ and, NADPH as an electron donor. Also, iNOS needs to form dimers to be functionally active and homodimerization requires binding of $\mathrm{L}$-arginine, heme and $\mathrm{BH}_{4}(68)$.

\section{Compartmentalized NO Signaling}

Recent data suggests that iNOS activity, although sustained, is subject to regulation within mammalian cells and at least 11 different proteins have been reported to bind to iNOS (calmodulin, caveolin-1, -2 , and -3, NAP110, kalirin, Rac1, Rac2, $\alpha$-actinin 4, EBP50 and PDZK1) $(69,70)$. Besides, some of these interactions can govern the subcellular location of the enzyme $(69,70)$. iNOS harbors the PDZ-domain recognizing motif SAL at its carboxyl-terminal and therefore, can tether onto PDZ-scaffolds. iNOS binding to EBP50 and CAP-70 are PDZ-mediated interactions and have been shown to be critical for polarized expression (at the apical membrane) of iNOS and vectorial NO synthesis $(69,70)$. NO being gaseous in nature is quite a diffusible molecule and, using cell culture models, it has been estimated that the diffusion of NO would be in the range of several $100 \mu$ meters (71). However, these calculations cannot be applied to complex biological systems as NO encounters numerous diffusion barriers inside the cell which include interaction with NO-responsive targets, quenching of NO by superoxides to form peroxynitrites, the partitioning of NO between polar and apolar media and exposure to cholesterol rich biomolecules (71). NO-mediated responses concord with the varied clinical stimuli so that the ensuing effects are diverse. This concludes that NO-generated responses can be compartmentalized which would involve selective localization of NOSs and the proximity of NO-responsive targets (71). Endothelial NOS was found to be localized mainly on the golgi apparatus and membrane caveole leading to highly confined S-nitrosylation of the target proteins (72). In certain physiological situations, certain receptors can stimulate NO synthases. For example, the proinflammatory peptides kinins stimulate the synthesis of NO that involves physical interaction of endothelial NOS with kinin $B_{1}$ and $B_{2}$ receptors (73). Estrogen upon binding to the plasma membraneassociated estrogen receptors can excessively stimulate NO generation from eNOS (74). Exclusive expression of iNOS on the apical membranes of the polarized epithelial cells suggests that the luminal membrane would be more enriched with NO and NO-dependent signaling pathways.

The most notable NO-mediated effect inside the cell is the stimulation of the cGMP synthesizing enzyme soluble guanylate cyclase (sGC) (75). NO binds to the attached prosthetic heme group present in $\mathrm{sGC}$ and induces conformational changes that stimulate the enzyme activities. In this fashion, $\mathrm{NO}$ can also regulate the amounts of cGMP inside the cell. It is also known that NO can stimulate different subtype of sGCs generating compartmental cGMP which regulates distinct receptors in the neuronal cells to regulate long-term potentiation and neurotransmitter release critical for synaptic plasticity (76). Also, if NO can be compartmentalized, it would also lead to localized cGMP generation which can be potentially important in conditions like IBD which has remarkably increased levels of iNOS. cGMP is an important cellular messenger and can 
regulate fluid homeostasis across the epithelial cells lining small intestine and colon (77). cGMP can stimulate CFTR $\mathrm{Cl}^{-}$channel function through kinase (PKA or PKG)-mediated phosphorylation of the channel (77). The membrane-bound cGMP-dependent protein kinase (cGK) type II which is predominantly expressed in intestinal epithelial cells colocalizes with CFTR and phosphorylates the channel to regulate its activity (78). Whether microdomain cGMP generated as a consequence of NO-cGMP pathway would play a role in CFTR regulation has not been elucidated before.

\section{Role of CFTR in Health and Disease}

\section{Cystic fibrosis}

CFTR was identified as a gene mutated in the pernicious autosomal recessive genetic disorder cystic fibrosis (CF). CF is most prevalent among Caucasians and one in every 3500 babies in USA would have CF. It is estimated that around 75,000 people in the world suffer from CF. CF patients suffer from severe exocrine insufficiency causing an obstructed mucosa especially in the lung airways. Sticky and thick mucus accumulates in the lung which favors colonization of opportunistic bacteria including Pseudomonas aeruginosa, Burkholderia cepacia, Staphylococcus aureus and Haemophilus influenza (35). P. aeruginosa forms biofilms composed of macrocolonies which are resistant to anti-microbial agents. Also, inflammatory responses get triggered accompanied by reduced levels of anti-inflammatory cytokines and proteases. The chronic infectious condition of the lung with the persistent deterioration of lung function becomes the major cause of morbidity and mortality of CF patients. Other complications associated with CF include fibrotic pancreas, pancreatic insufficiency, potentially fatal condition of the gut (meconium ileus) in about $10 \%$ of the patients and male infertility. The best biomarker of $\mathrm{CF}$ with a correlated involvement of CFTR in the disease is $\mathrm{Cl}^{-}$. Therefore, in order to confirm if a patient has CF, salt concentration in the sweat less than $60 \mathrm{mmol} / \mathrm{L}$ is considered as intermediate- or non-CF. This is based on the hypothesis that CFTR in sweat glands absorb $\mathrm{Cl}^{-}$. In case of a defective CFTR as in $\mathrm{CF}, \mathrm{Cl}^{-}$fails to be absorbed properly and therefore, forms salt. In the cases where the sweat $\mathrm{Cl}^{-}$is normal or intermediary but the individual has CF-manifestations or bears a family history of CF, it is necessary to confirm the possibility of having $\mathrm{CF}$ by measuring nasal potential difference (NPD) (79). In CF, luminal surfaces have more negative charge either because of the overly active $\mathrm{Na}^{+}$absorption in the absence of CFTR or the inability to absorb $\mathrm{Cl}^{-}$. Usually, the basal PD of CF patients is approximately two-to three-folds higher than normal individuals (80)

There are around 1900 CFTR mutations known today but not every mutation leads to CF (81). Any CFTR mutation which results in defective protein synthesis, halted processing, defective trafficking, block in regulation, altered conductance or reduced synthesis of CFTR can cause CF (82). F508Del mutation in CFTR is the most common CF-causing mutation accounting for about $70 \%$ of the CF-underlying defects. F508Del mutation causes CFTR to be misfolded and recognized by ER-associated degradative 
machinery. Now other defects associated with F508Del have been recognized which include altered conductance, mislocalization, impaired post-ER trafficking, poor subapical membrane retrieval, markedly low-stability at the membrane and decreased mRNA levels (83). Newly recognized defects with F508Del-CFTR include thermal destabilization caused by this mutation and the impaired inter-domain interactions between NBD1 and membrane spanning domains (84-86).

Because F508Del-CFTR is known to possess partial $\mathrm{Cl}^{-}$conductance once it reaches the cell surface, several CF therapies have been based on the potentiation of a small population of F508Del-CFTR present at the cell surface. The CF-potentiator VX770 is clinically approved for CF-patients carrying G551D-CFTR (exhibits a locked $\mathrm{Cl}^{-}$ conductance) and its efficacy is being evaluated in F508Del homozygous mutant CFpatients (87). Other therapies have focused on lifting the processing defects to increase F508Del-CFTR number at the plasma membrane. However, the clinical trial of CFcorrector VX-809 which increases the F508Del-CFTR population reaching at the surface failed to show promising lung improvement in CF-patients although sweat $\mathrm{Cl}^{-}$ concentration was reduced with VX-809 treatment (88).

Interestingly, it is not only the deficiency of CFTR which leads to a pathological condition as CFTR might contribute to the enlargement of renal cysts in individuals with polycystic kidney disease and hyperactivity of CFTR in the gut causes infectious diarrhea $(89,90)$.

\section{Role of CFTR in fluid secretion and diarrhea}

Approximately eight liters of fluid is secreted into the intestine everyday (77). Regulation of fluid secretion is critical as enzymes and solutes need aqueous media to diffuse and function and, also the mucosa needs to be lubricated for preventing frictional damage during motility (77). The key to fluid secretion is how several electrolyte transporters present on the gut epithelia are regulated. Secretion of selective electrolytes and solutes into the lumen sets the osmotic gradient for the movement of water. It was mostly believed that water takes the paracellular route, however recent evidences indicate the possibility of transcellular water transport. There is a simple mechanics, very wellunderstood today, on how epithelial ion transporters function on the intestinal surface to regulate water movement.

It is now accepted that together with $\mathrm{Na}^{+}$absorption, $\mathrm{Cl}^{-}$secretion constitutes the predominant driving component of fluid secretion (77). The electroneutral transporter $\mathrm{Na} / \mathrm{K} / 2 \mathrm{Cl}$ (NKCC) on the basolateral membrane takes up $\mathrm{Cl}^{-}$into the cell which sets the electrochemical potential for $\mathrm{Cl}^{-}$. When the $\mathrm{Cl}^{-}$channels on the apical surface, (predominantly CFTR) open, $\mathrm{Cl}^{-}$gets transported into the lumen. $\mathrm{Na}^{+} \mathrm{K}^{+}$-ATPase located on the basolateral surface functions as an electrogenic transporter to maintain the thrust for $\mathrm{Cl}^{-}$secretion out of the cell. In order to maintain electroneutrality, $\mathrm{Na}^{+}$is secreted into the lumen via a paracellular pathway resulting in an osmotic flux of water. The schematics of the entire process are shown in Figure 2-4. 


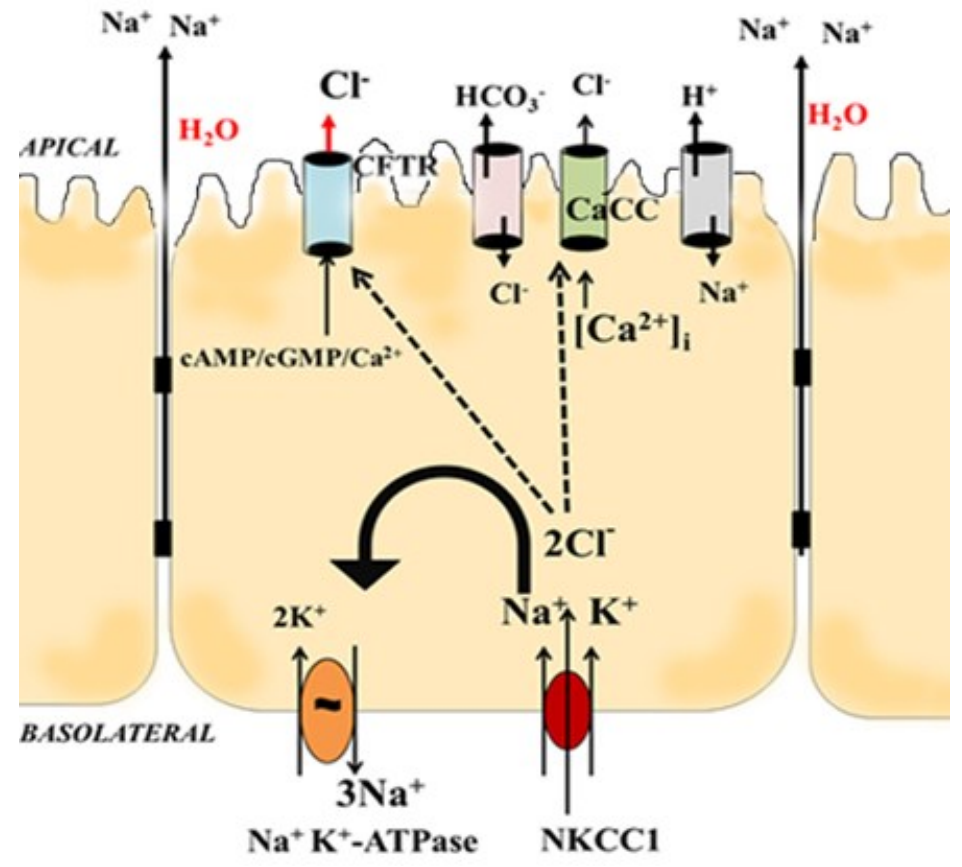

Figure 2-4. Fluid transport model in a polarized intestinal epithelial cell

Concerted actions of various transporters located on the apical and basolateral membranes of polarized gut epithelial cells regulate fluid transport. 
It is a well-known fact now that secretory and absorptive processes along the length of the intestine are highly asymmetric to the extent that the surface operating mechanisms will be different than those in the intestinal crypts (77). Still, relevance of CFTR-mediated $\mathrm{Cl}^{-}$secretion in regulation of fluid homeostasis is irrespective of the region of the gut and cannot be discounted. In $\mathrm{CF}$, the failure to secrete sufficient $\mathrm{Cl}^{-}$ leads to dehydrated and obstructed mucosa (meconium ileus) in $10 \%$ of the neonates, distal intestinal obstruction syndrome (DIOS), and chronic constipation in older CF patients (91).

Secretory diarrhea is a consequence of major electrolyte transport abnormalities in the gut mucosa. Colonization by certain pathogenic enterobacteria majorly E. coli, Vibrio cholerae, Yersinia enterocolitica, Shigella flexneri, and Salmonella typhimurium and their toxic secretagouges lead to secretory diarrhea (34). Given the fact that increase in intracellular levels of cAMP, cGMP or $\mathrm{Ca}^{2+}$ activates CFTR $\mathrm{Cl}^{-}$channel function, any molecular agent which causes unregulated increase in the levels of these messengers would surpass the regulatory threshold on CFTR and cause fluid dysregulation such as diarrhea. CFTR heterozygosity conferred resistance to diarrhea induced by CTX, an enterotoxin from Vibrio cholera, which underscored the direct involvement of CFTR in causing secretory diarrhea (90). In this study, CF-mice failed to have any secretory phenotype in response to CTX challenge. The underlying mechanism of CTX-induced diarrhea is irreversible ADP-ribosylation of G-protein $\mathrm{G} \alpha_{\mathrm{s}}$ by CTX which locks it in the active GTP-bound state. Constitutively active $\mathrm{G} \alpha_{\mathrm{s}}$ stimulates $\mathrm{AC}$ in an unregulated manner which pools the cell with cAMP. Excessive cAMP causes overstimulation of CFTR activity and downregulation of $\mathrm{Na}^{+}$absorption culminating into excessive flow of water into the gut and diarrhea. A question of interest would be whether these enterotoxins can be used as a therapy for CF patients to partially relieve meconium ileus? Enterotoxins will be effective in CF patients with the condition of meconium ileus provided they are able to stimulate CFTR-independent secretions. It is also known that cholera toxin cannot trigger any secretion in CF mice. It is to be noted that predisposition to meconium ileus is genetically determined and the predisposition of the CF patients to this phenotype is variable. Also, not all CF patients have meconium ileus implying that several other genomic loci than CFTR are also involved. A recent study reports that guanlyl cyclase $2 \mathrm{C}$ encoded by $G U C Y 2 C$ can be a likely modifier of meconium ileus associated with CF (92). Therefore, if the genes other than CFTR can be targeted that improves secretion, there is a possibility that meconium ileus can be effectively controlled.

Heat-stable enterotoxins from E. coli or $Y$. enterocolitica directly stimulate membrane-bound guanylyl cyclase C (GC-C) and enhance intracellular cGMP (93). CFTR activity gets stimulated by the efficient phosphorylation of the channel by membrane localized cGMP-dependent protein kinase II (cGKII) which in combination with hypoabsorption of $\mathrm{Na}^{+}$result in diarrhea $(94,95)$. Also, upregulated levels of iNOS and prostaglandin generating enzyme cyclooxygenase- 2 could enhance $\mathrm{Cl}^{-}$secretion and leakiness of epithelial junctions in a colon epithelial cell line challenged with enteroinvasive bacteria (96). This can be the possible mechanism of infectious diarrhea caused by these bacteria. 
Approximately 2.5 million children in the developing world die annually from infectious diarrhea and it accounts for $21 \%$ of the causes of death of children aged under 5 years (97). Interestingly, infectious diarrhea comes next to cardiovascular disorders as a major cause of mortality especially in the subsets of pediatric and elderly population. With the validation of CFTR involvement in many forms of secretory diarrhea, therapies to control diarrhea are now focused on developing drugs to block CFTR $\mathrm{Cl}^{-}$secretion in the patients some of which are already known to be effective. The plant latex derivative SP-303 was demonstrated to attenuate CTX-induced CFTR-dependent secretion in vitro and its naturally occurring counterpart, derived from the latex of the plant Croton lechleri has always been in use by natives of South America to control various kinds of watery diarrheas. Based on the effects of LPA in downregulating CFTR function and ameliorating diarrheal phenotype of CTX-injected mice, an LPA-rich diet can effectively control certain forms of diarrhea. Cesinex ${ }^{\circledR}$, a tannic acid based medical food is prescribed for diarrheal episodes in USA and could successfully attenuate CTX-induced diarrhea in mice (98). It is known that egg albumen has high amounts of LPA. Based on the aforementioned studies, a formulation combining tannic acid and egg albumen can exhibit improved anti-diarrheal properties. Also, MRP4 which couples closely with CFTR and regulates its function in physiological and pathophysiological situations can represent another target for therapeutic interventions of diarrheal disorders.

With a major contribution of CFTR in regulation of fluid secretion across the gut mucosa, there is an imperative interest in the contribution of CFTR in other diarrheal disorders including, inflammatory bowel disorders, irritable bowel disorders and diverticulitis. However, the exact role of CFTR in contributing to diarrhea in these disorders has not been clearly elucidated. This aspect would define the role of CFTR under a pathological situation.

\section{Inflammatory Bowel Disease}

Inflammatory bowel disorders (IBD) represent chronic relapsing inflammatory condition of the gastrointestinal tract (GI) and are broadly categorized into ulcerative colitis (UC) and Crohn's disease (CD). UC extends proximally from the rectum and is restricted to the colon mucosa while $\mathrm{CD}$ is transmural and can affect any part of the GI tract (99). IBD patients suffer from diarrhea, abdominal pain, and weight loss and with long persistence are predisposed to an increased risk of developing colorectal cancer (100). More than one million people world-wide suffer from IBD and it is also the major cause of morbidity in the western world. The etiology of IBD is understood to be multifactorial and the precise onset of IBD is still not established (101). In particular, a compromised epithelial barrier in the intestine of genetically predisposed IBD patients supposedly cause commensal intestinal flora to trigger innate inflammatory response, thus leading to the disease. Therefore, IBD is often described as an autoimmune disorder.

Diarrhea is the most common and distressing symptom in IBD patients often 'bloody' with a 50\% consistency in acute flare-ups of CD and almost $100 \%$ of UC patients (102). IBD patients have varied diarrheal manifestations leading to an unresolved 
conclusion about the mechanism of diarrhea in IBD. It is critical to define the precise pathophysiologic mechanisms of diarrhea in IBD patients to effectively control it.

\section{Electrolyte transport abnormalities in IBD}

It is an accepted fact that IBD patients have disturbed electrolyte transport. Inflammatory secretagogues present in the supernatant of inflamed human colonic mucosa could stimulate electrogenic $\mathrm{Cl}^{-}$secretion in rat colonic mucosa in Ussing chambers which suggested that $\mathrm{Cl}^{-}$secretion is probably altered in IBD (103). However, it did not reconcile with the observed diminished intrinsic responsiveness of the mucosa to the secretory stimuli and loss of the lumen-negative potential difference (PD) $(103,104)$. There were reports on the reduced cAMP-mediated secretory response of the inflamed mucosal tissue which may not necessarily have to do with the defects in the AMP pathway or cAMP-responsive proteins but with the type of cAMP-elevating ligands or the receptor profile of the ligands used (103). Alterations in the expression and function of CFTR in IBD have not been determined with reproducibility (103). Genetically, it has been determined that the carriers of the loss-of-function mutation of CFTR, F508Del are 8 -times less likely to develop IBD (105). However, conclusive data on the phenotypic role of $\mathrm{Cl}^{-}$secretion and CFTR in IBD-associated electrolyte abnormalities is lacking. CFTR-function is the rate-limiting determinant of luminal fluid levels also considering the fact that it regulates the function of several other transporters and channels including $\mathrm{Na}^{+} / \mathrm{H}^{+}$exchangers, anion exchangers, sodium-bicarbonate transporters, $\mathrm{K}^{+}$channels, epithelial sodium channel (ENaC), ATP-release channels, gap-junctional channels and aquaporins $(47,106)$. The regulation mostly happens in the form of large macromolecular signaling complexes of CFTR. Therefore, it is critical to precisely define the role of CFTR in IBD-associated diarrhea and if macromolecular complexes of CFTR would play a role in it.

\section{iNOS in inflammatory bowel disease}

Pertaining to the blatant inflammatory profile of IBD, the therapeutic approaches for IBD have been mostly based on targeting inflammatory mediators. iNOS which has been found to be elevated in IBD mucosa has also been studied as a therapeutic target in animal models of experimental colitis. Colonic mucosa of IBD patients have two to fivefold higher amounts of NO compared to those in the controls or in the mucosa of remitting UC patients. This correlates with the increased expression and activity of iNOS $(107,108)$. The correlation between iNOS activity and disease activity has been less consistent for CD (109). iNOS has also been linked to UC disease susceptibility in the Spanish population subset (110). iNOS in contrast to other NOS isoforms causes sustained NO production in the $\mu$ molar range (75). Excessive NO inside the cell causes oxidative stress (by forming peroxynitrites-ONOO${ }^{-}$) and may also lead to the formation of potential carcinogens $(75,111,112)$. Oxidative stress has been shown to substantially lead to mucosal injury that compromises the barrier integrity rendering it leaky.

Excessive $\mathrm{NO} / \mathrm{ONOO}^{-}$activity has been demonstrated to cause cytoskeletal nitration and 
oxidation in the intestinal mucosa from IBD patients (113). The toxic megacolon, a potentially lethal manifestation of $\mathrm{UC}$, has also been associated with the presence of large amounts of iNOS in the colonic muscularis propria and it was also reported that administration of iNOS inhibitor L-NAME helped patients recover from megacolon (75, 114). The drug named mesalamine given to the IBD patients can scavenge peroxynitrites generated as a consequence of sustained iNOS activity and can attenuate apoptosis (75).

\section{Animal models of inflammatory bowel disorders}

Inflammatory bowel disease has a complicated etiology and more than 60 animal models are available to study IBD. These IBD models have provided significant contributions in dissecting out various mechanisms contributing to the development of the disease and further channel it into therapeutic interventions of IBD. IBD mouse models have been primarily classified into gene knockout (KO) models, transgenic models, chemically-induced colitis models and adoptive cell transfer models (115). Induction of intestinal inflammation in mice by chemical irritation is one of the most commonly used approach because it is simple, inexpensive, and less time-consuming and the onset, duration, and severity of the inflammation can be easily controlled (116). The dextran sulphate sodium (DSS) and trinitrobenzene sulfonic acid (TNBS) induced colitis are the most popular animal models of chemically-induced mucosal inflammation (116). Several studies have validated the evaluations of DSS-induced colitis model to be extended to translational studies of human. This model gives the flexibility of inducing acute, chronic, or relapsing conditions of colitis by changing the concentration and duration of administration of DSS (116). DSS is a sulfated polysaccharide permeable to mucosa and causes epithelial injury (116). Animals are fed continuously with $2-5 \%$ DSS in the drinking water for short period (4-9 days) in order to induce acute colitis and the symptoms usually appear from the $4^{\text {th }}$ day onwards (117). It is considerably important to take into account the molecular weight of DSS to be used as it governs the severity, extent, localization of colitis and also colitis-induced dysplastic lesions (116). Clinical manifestations of acute DSS colitis closely mimic IBD-like symptoms and include weight loss, diarrhea, rectal bleeding; however, in many cases animals eventually die $(75,116$, 117). Typical histopathology of acute DSS-colitis is characterized by mucosal damage, cryptitis, crypt abscesses and neutrophil infiltration of lamina propria $(116,117)$. As the DSS colitis gets chronic, the inflammatory profile becomes less representative of IBD and partially loses histopathological features of large bowel in IBD (116). Based on the numerous advantages that DSS colitis model offers and the close resemblance it bears to IBD, the model can prove invaluable in order to study the epithelial ion transport abnormalities in colitis and the contribution of selective channels in causing diarrhea.

TNBS is dissolved in ethanol and the ethanol enema causes disruption of the mucosal barrier and the TNBS chemical penetrates into virtually all layers of the intestine. Consequently, granuloma infiltrates can be observed transmural. The colitis seen in this model is a Th type-1 response which mimics a CD background (115). 


\section{Specific Aims}

The unifying hypothesis of my study is that excessive amounts of inducible nitric oxide synthase (iNOS) are expressed at the apical plasma membrane of the gut epithelia in inflammatory bowel disease where iNOS physically and functionally couples with cystic fibrosis transmembrane conductance regulator (CFTR; a cGMP-activated $\mathrm{Cl}^{-}$ channel) and potentiates its $\mathrm{Cl}^{-}$channel function through stimulation of NO-cGMP pathway. Schematics of the hypothesis are shown in Figure 2-5.

The specific aims of the study were to answer the following questions:

- Is iNOS overexpressed and does it exhibit highly polarized expression in the gut epithelial cells of IBD-affected tissue?

- Is iNOS involved in a high affinity interaction with PDZ-protein NHERF2 at the apical plasma membrane of pathophysiological relevance?

- $\quad$ Does NHERF2 bridge CFTR and iNOS in a form of macromolecular signaling complex?

- Does iNOS contribute to microdomain cGMP at the plasma membrane via the potentiation of the NO-cGMP pathway?

- $\quad$ Does iNOS-induced compartmentalized cGMP potentiate CFTR Cl- channel function at the plasma membrane in IBD?

- $\quad$ Does inhibition of CFTR and iNOS activities confer resistance to colitisassociated diarrhea?

- $\quad$ Does compromise of the macromolecular complex among CFTR, NHERF2 and iNOS ameliorate diarrhea in colitis models? Is diarrhea attenuated in DSS and TNBS colitis models in a Nherf $2^{-}$genetic background? 


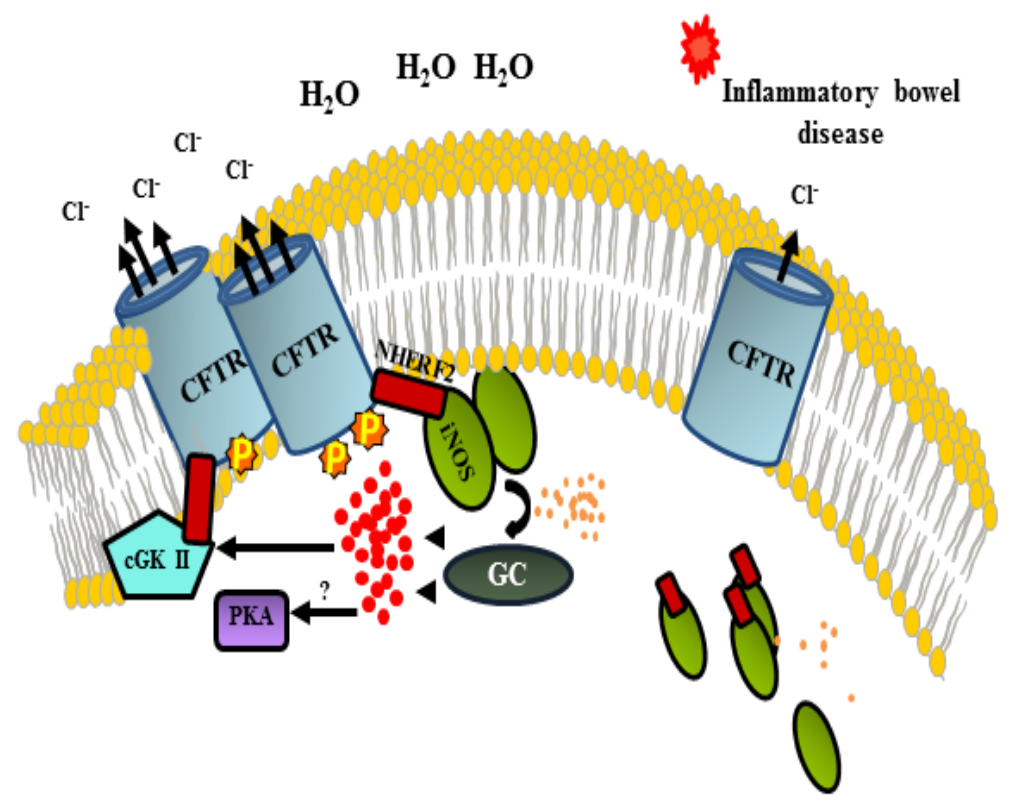

Figure 2-5. Model of the hypothesis

The molecular machinery of how a macromolecular complex of CFTR and iNOS contributes to diarrhea in inflammatory bowel disease. 


\section{CHAPTER 3. METHODS}

\section{Reagents}

Zaprinast was purchased from Enzo Life Sciences (Farmingdale, NY), and DSP was obtained from Pierce (Thermo Fisher, Rockford, IL). Bacterial LPS was purchased from Sigma-Aldrich (St. Louis, MO), and recombinant human TNF- $\alpha$, IL-1 $\beta$, and IFN- $\gamma$ were obtained from BD Biosciences (Franklin Lakes, NJ). Dextran sodium sulfate (DSS; MW 36,000-50,000) was purchased from MP Biomedicals (Irvine, CA).

\section{Cell Culture and Transfections}

HEK-293 and HT29-CL19A cells were cultured in DMEM-F12 and DMEM high glucose media, respectively (Invitrogen, Carlsbad, CA) containing 10\% serum and $1 \%$ penicillin/streptomycin and maintained in a $5 \% \mathrm{CO}_{2}$ incubator at $37^{\circ} \mathrm{C}$. Lipofectamine 2000 (Invitrogen, Carlsbad, CA) was used to express various plasmids in HEK-293 cell lines according to the manufacturer's instructions. Stable cell lines were generated by lentiviral transfection and selected with puromycin $(2 \mu \mathrm{g} / \mathrm{ml})$.

\section{Purification of Flag- and HA-iNOS, and GST-His-S-and His-S-iNOS-C-tail Proteins}

HEK-293 cells stably expressing hemagglutinin (HA)-iNOS were plated on 100 $\mathrm{mm}$ dishes, and grown till 100\% confluency. The cells were then washed with PBS twice and lysed with PBS containing $0.2 \%$ Triton-X-100 and protease inhibitor cocktail (phenylmethylsulfonyl fluoride $1 \mathrm{mM}$, pepstatin-A $1 \mu \mathrm{g} / \mathrm{ml}$, leupeptin $1 \mu \mathrm{g} / \mathrm{ml}$, aprotinin $1 \mu \mathrm{g} / \mathrm{ml})$. The cell lysate was mixed on a shaker for $10 \mathrm{~min}$ at $4^{\circ} \mathrm{C}$ and centrifuged for 10 min at 13,000 rpm. The supernatant containing total protein was immunoprecipitated using $\alpha$-Flag or $\alpha$-HA beads overnight. The beads with bound protein were washed and the protein was eluted with $100 \mathrm{mM}$ glycine $(\mathrm{pH}$ 2.2) and quickly neutralized with 150 $\mathrm{mM}$ tris (pH 8.8). The eluted protein was subjected to SDS-PAGE on $4-15 \%$ gel (BioRad, Hercules, CA) and transferred to PVDF membrane and either visualized with Gel Code Blue or immunoblotted with $\alpha$-Flag-HRP or $\alpha$-HA-HRP antibody.

For the purification of GST-His-S- or His-S-tagged iNOS-C-tail, pET-41- and pTriEx-4-iNOS-C-tail constructs were used to transform BL21 and Origami competent cells, respectively. Bacterial cultures $(1 \mathrm{~L})$ were grown and the protein synthesis was induced with IPTG $(0.2 \mathrm{mM})$ for $4 \mathrm{~h}$. For GST-tagged and His-tagged proteins lysis buffer comprising of $50 \mathrm{mM}$ Tris, $1 \mathrm{mM}$ EDTA and $10 \%$ Sucrose $\mathrm{pH} 7.2$ and resuspension buffer containing $25 \mathrm{mM}$ HEPES-KOH, $400 \mathrm{mM} \mathrm{KCl}$ and $5 \mathrm{mM} \beta$ mercaptoethanol (added fresh) respectively, was used followed by addition of lysozyme $(1 \mathrm{mg} / \mathrm{ml})$ prepared in lysis or resuspension buffer for $30 \mathrm{~min}$ at $4^{\circ} \mathrm{C}$. Next, $2.1 \%$ Triton$\mathrm{X}-100$ was added to the lysate and mixed for another $30 \mathrm{~min}$ at $4^{\circ} \mathrm{C}$. Lysate was transferred to clean centrifuge tubes and spun at $15,000 \mathrm{rpm}$ for $30 \mathrm{~min}$ at $4^{\circ} \mathrm{C}$. To the 
clear supernatant $100 \mu 1$ of Glutathione beads (Thermo Scientific, Waltham, MA) or Talon-beads (Clontech, Mountain View, CA) were added as appropriate and mixed for 2$3 \mathrm{~h}$ at $4^{\circ} \mathrm{C}$. Glutathione beads and Talon beads containing proteins were washed threefour times with PBS $0.2 \%$ Triton-X-100 and resuspension buffer; respectively in the presence of protease inhibitors. GST-His-S-iNOS-C-tail and His-S-iNOS-C-tail proteins were eluted with $20 \mathrm{mM}$ glutathione and $200 \mathrm{mM}$ imidazole, respectively for 1-4 times. Protein concentrations of the samples were quantified using Bradford assay or by direct absorbance at $280 \mathrm{~nm}$.

\section{PDZ-array}

Purified GST-NHERF1/NHERF2/PDZK1 PDZ proteins were immobilized on nitrocellulose membrane in various concentrations using a slot blot applicator. Purified GST-His-S-iNOS-C-tail (100 ng) protein was added to the membrane in $0.1 \%$ Tween 20 , $2 \%$ BSA with protease inhibitors for overnight binding at $4{ }^{\circ} \mathrm{C}$. Next day, the membrane was washed extensively with TBS $0.1 \%$ Tween- 20 and probed with S-HRP antibody.

\section{Pull-down Assay}

HEK-293 cells overexpressing HA-iNOS were lysed in lysis buffer (PBS $0.2 \%$ Triton X-100 + protease inhibitors). Cell lysates were mixed for $15 \mathrm{~min}$ followed by centrifugation at $15,000 \mathrm{~g}$ for $10 \mathrm{~min}$ at $4^{\circ} \mathrm{C}$. GST-NHERF 2 was added and mixed at $4^{\circ} \mathrm{C}$ with the clear supernatant for 30-60 min. Glutathione beads $(20 \mu \mathrm{l})$ were then added and mixed overnight at $4^{\circ} \mathrm{C}$. Thereafter, the mixtures were spun at $3000 \mathrm{rpm}$ for $2 \mathrm{~min}$, and the beads were washed three times with the same lysis buffer before the proteins were eluted from the beads with the sample buffer (containing $2.5 \% \beta$-mercaptoethanol). Eluates were separated on 4-15\% gel and immunoblotted with mouse monoclonal $\alpha$ iNOS antibody (BD Biosciences, Franklin Lakes, NJ).

In the next set of assays, HEK-293 cells overexpressing iNOS and iNOS- $\triangle \mathrm{PDZ}$ or mucosal scrapings from DSS-treated and untreated colons were lysed in lysis buffer (PBS containing $0.2 \%$ Triton X-100 and protease inhibitors), mixed for $15 \mathrm{~min}$ followed by centrifugation at $15,000 \mathrm{rpm}$ for $10 \mathrm{~min}$ at $4^{\circ} \mathrm{C}$. GST-NHERF2 was added and mixed at $4^{\circ} \mathrm{C}$ with the clear supernatant $(\sim 500 \mu \mathrm{g})$ for 30-60 min. Glutathione beads $(20 \mu \mathrm{l})$ were then added and mixed overnight. Thereafter, the mixtures were spun at $3000 \mathrm{rpm}$ for 2 $\mathrm{min}$, and the beads were washed three times with the same lysis buffer before the proteins were eluted from the beads with the sample buffer (containing 2.5\% $\beta$-mercaptoethanol). For the tissue pull-down, proteins were eluted with $100 \mathrm{mM}$ glycine $(\mathrm{pH} 2.2)$ and quickly neutralized with $150 \mathrm{mM}$ Tris ( $\mathrm{pH} 8.8$ ). Eluates were separated on $4-15 \%$ gel and immunoblotted with mouse monoclonal $\alpha$-iNOS antibody and rabbit polyclonal 1314 CFTR antibody. 


\section{Co-immunoprecipitation}

HA-iNOS-HEK-293 and control-HEK-293 cells were incubated with the crosslinker dithiobis succinimidyl propionate (DSP, $1 \mathrm{mM}$ ) for $10 \mathrm{~min}$ at $37^{\circ} \mathrm{C}$. Cells were then lysed using lysis buffer-1X PBS, containing $0.2 \%$ Triton-X-100 and protease inhibitors and the clear supernatant was subjected to immunoprecipitation by using HAbeads (Sigma-Aldrich, St.Louis, MO) for overnight at $4^{\circ} \mathrm{C}$. The cross-linked complex was eluted with $100 \mathrm{mM}$ glycine (pH 2.2) and quickly neutralized with $150 \mathrm{mM}$ Tris $(\mathrm{pH}$ $8.8)$. The eluted proteins were mixed with the sample buffer $(5 X$; containing $1 \% \beta$ mercaptoethanol), denatured, subjected to SDS-PAGE, transferred to PVDF membrane, and immunoblotted using rabbit polyclonal $\alpha$-NHERF2 antibody.

\section{Direct Sensitized Fluorescence Resonance Energy Transfer Microscopy and Data Analysis}

For direct sensitized emission FRET, HEK-293 cells were transiently transfected with pECFP-iNOS, YFP-CFTR, Yellow-NHERF2, pCMV-HA-NHERF2 either singly or in combination using Lipofectamine 2000 (Invitrogen, Carlsbad, CA). Single transfected cells were used to acquire CFP- or YFP- only images for bleedthrough calculations. The cells were maintained at low density (20-40\% confluence) on a cover slip bottom dish (MatTek; Ashland, MA), washed twice with warm HBSS and $2 \mathrm{ml}$ of HBSS was added. Cells were imaged in a dark room at $37^{\circ} \mathrm{C}$. Corrected FRET was calculated on a pixel-bypixel basis for the entire image by using equation: FRETc $=$ FRET $-(0.5 \times \mathrm{CFP})-(0.06 \times$ YFP), where FRET, CFP, and YFP correspond to background-subtracted images of cells co-expressing CFP-iNOS and YFP-CFTR/Yellow-NHERF2 acquired through the FRET, CFP, and YFP channels, respectively. The factors 0.5 and 0.06 are the fractions of bleedthrough of CFP and YFP fluorescence, respectively, through the FRET filter channel. The corrected FRET (FRETc) was normalized with donor CFP intensity (FRETc/CFP), yielding the normalized corrected FRET (N-FRETc), and the intensity of N-FRETc images was presented in monochrome mode, stretched between the low and high renormalization values, according to an intensity to color mapped lookup table with black indicating low values and white indicating high values. All calculations were performed using the Channel Math and FRET modules of the SlideBook 4.2 software (Intelligent Imaging Innovations; Denver, CO).

\section{AlphaScreen ${ }^{\mathrm{TM}}$ Assay for iNOS-NHERF2 Interaction}

We used AlphaScreen ${ }^{\mathrm{TM}}$ (Amplified Luminescent Proximity Homogeneous Assay) to study the interaction between purified full-length GST-NHERF2 and biotin-iNOS-Ctail peptide (last 10 amino acids), using AlphaScreen ${ }^{\mathrm{TM}}$ GST Detection Kit (PerkinElmer, Waltham, MA). In brief, starting from $300 \mu \mathrm{M}$ concentration, biotin-iNOS-C-tail peptide was serially diluted (in 1/2 log dilution series) in assay buffer [1x HEPES, $0.1 \%$ BSA, $0.05 \%$ Tween 20 (v/v), pH 7.2] containing GST-NHERF2 (100 nM final concentration). For the interaction between His-S-iNOS-C-tail and GST-NHERF2, similar procedure was 
followed with the starting concentration of $100 \mathrm{nM}$ for His-S-iNOS-C-tail with progressive dilution and $100 \mathrm{nM}$ final concentration for GST-NHERF2. The resulting solutions were incubated at room temperature for $30 \mathrm{~min}$. Each sample solution $(15 \mu \mathrm{l})$ was transferred to a white opaque 384-well microplate (OptiPlateTM-384; PerkinElmer, Waltham, MA) in triplicates and into which anti-GST acceptor beads $(5 \mu 1,20 \mu \mathrm{g} / \mathrm{ml}$ final concentration) were added and incubated for $30 \mathrm{~min}$ at room temperature.

Streptavidin donor beads $(5 \mu \mathrm{L} ; 20 \mu \mathrm{g} / \mathrm{ml}$ final concentration) were then added and incubated for $2 \mathrm{~h}$ at room temperature. Anti-GST acceptor beads $(5 \mu \mathrm{l}, 20 \mu \mathrm{g} / \mathrm{ml}$ final concentration) and Nickel chelate donor beads ( $5 \mu \mathrm{l}, 20 \mu \mathrm{g} / \mathrm{ml}$ final concentration) were used for GST-His-S-iNOS-C-tail and GST-NHERF2 interaction. The plate was read on FLUOstar-Omega plate reader (Ortenberg, Germany).

The specificity of the observed AlphaScreen ${ }^{\mathrm{TM}}$ binding signals between biotiniNOS-C-tail peptide and GST-NHERF2 protein was verified by AlphaScreen ${ }^{\mathrm{TM}}$ competition assay. The assay procedure is similar to the association assay described above except that a non-biotinylated iNOS peptide was serially diluted in the assay buffer starting with $1 \mathrm{mM}$ final concentration and mixed with GST-NHERF2 (100 nM final concentration).

\section{Macromolecular Complex Assembly}

GST-His-S-iNOS-C terminal 112 a.a. fusion protein $(20 \mu \mathrm{g})$ was mixed with various amounts of GST-NHERF2 $(0-20 \mu \mathrm{g})$ at $4^{\circ} \mathrm{C}$ for $1 \mathrm{~h}$, followed by mixing with Sepharose beads $(20 \mu \mathrm{l})$ for another $1 \mathrm{hr}$. This step, which is called pairwise binding, was done in $200 \mu \mathrm{l}$ of lysis buffer (1X PBS- $0.2 \%$ Triton X-100 + protease inhibitors). The complex was washed twice with the same buffer and allowed to incubate overnight with $0.5 \mu \mathrm{g}$ purified Flag-CFTR at $4^{\circ} \mathrm{C}$ with constant mixing. The complex was washed extensively with lysis buffer, eluted with sample buffer, and immunoblotted with FlagHRP antibody.

\section{DSS-induced Colitis Murine Model}

C57BL/6 male mice, 7-8 weeks old, were fed with 5\% DSS in drinking water for 7 days and euthanized later. The control mice were fed with autoclaved water.

In order to evaluate the contribution of CFTR in DSS-induced diarrhea, CFTR inhibitor CFTR $_{\text {inh- }}-172(150 \mu \mathrm{g} / \mathrm{kg})$ prepared in saline was administered intraperitoneally on the $5^{\text {th }}$ day of DSS-treatment following appearance of symptoms of colitis and was given twice per day for 3 days. On the $8^{\text {th }}$ day mice were sacrificed and colon tissue was removed and assessed for total length, rectal bleeding score using ColonScreen-ES (Helena laboratories, Beaumont, TX), wet/dry weight ratio and histological examination. Dry to wet weight ratio was calculated by drying fecal pellets from the colon at $50^{\circ} \mathrm{C}$ overnight. 
iNOS inhibitor L-NIL (N6-(1-Iminoethyl)-L-lysine dihydrochloride) (Cayman Chemical, Ann Arbor, MI) known to be highly selective for iNOS was administered intraperitoneally at a concentration of $10 \mathrm{mg} / \mathrm{kg} / 12 \mathrm{~h}$ for 6,4 and 2 days in three different mouse groups treated with 5\% DSS containing four animals per group. Saline was administered in the control animals.

\section{TNBS Colitis Murine Model}

TNBS colitis was induced by a single rectal injection of $3.75 \mathrm{mg}$ of TNBS dissolved in 50\% ethanol-PBS solution into the descending colon of C57BL/6 male Nherf $2^{-/}$and Nherf $2^{+/+}$mice using a catheter inserted up to $3-4 \mathrm{~cm}$ into the anus. The volume of TNBS enema was kept at $100 \mu$ l. The mice were anesthetized with intraperitoneal injection of xylazine-ketamine solution $(50 \mathrm{mg} / \mathrm{kg})$. The animals were observed for 3 days with regular weight measurements, and afterwards euthanized for further assessment.

\section{Determination of Clinical Scores}

Stool consistency and the presence of occult blood were determined on day 7 of DSS treatment. Stool consistency for TNBS-colitis was determined on day 3. Briefly, stool scores were determined on the scale of 0 to 5 depending upon the looseness of the fecal matter with, $0=$ well-formed pellets that did not stick to the anus, $1=$ semi-formed stools that did not stick to the anus, $2-4=$ depending upon the fluidity of the fecal matter that did not stick to the anus and $5=$ non-standing liquid stool. Bleeding scores were determined as follows: $0=$ no blood, $1=$ positive hemoccult, $2-4=$ based on significance of visible blood traces in the stool, $5=$ gross rectal bleeding.

\section{Histopathology Scoring for DSS Colitis}

After day 7, the entire colon was excised to measure the length of the colon. Colons were washed, fixed in formalin and embedded in paraffin. Tissue sections were stained with hematoxylin \& eosin (H\&E). Histology was scored by a pathologist in a blinded fashion based on combination of inflammatory cell infiltration (score $0-3$ ) and tissue damage (score $0-3$ ) as described previously (118). The presence of occasional inflammatory cells in the lamina propria was scored as 0 , increased numbers of inflammatory cells in the lamina propria was assigned score 1, confluence of inflammatory cells extending into the submucosa was scored as 2, and transmural extension of the infiltrate was scored as 3 . For tissue damage, no mucosal damage was scored as 0 , lymphoepithelial lesions were scored as 1 , surface mucosal erosion or focal ulceration was scored as 2 , and extensive mucosal damage and extension into deeper structures of the bowel wall was scored as 3 . The combined histological score ranged from 0 (no changes) to 6 (extensive infiltration and tissue damage). 


\section{Human Colon Sections}

We used 5-micron thick sections of normal and IBD-affected, paraffin-embedded human gut tissues. These slides were made available by the Tissue Service Core, Pathology Department (University of Tennessee Health Science Center) under the IRBapproved protocol 05-03854-XM. These samples and preexisting tissue from normal and IBD individuals were de-identified.

\section{Human mRNA Expression Data Set}

The mRNA expression data set was downloaded from the NCBI Gene Expression Omnibus database (accession number GSE1710) (119). This data set contains 31 samples generated from 10 patients with CD, 10 patients with UC, and 11 normal controls. Data sets were log-transformed and then normalized by Zipf's law. The normalized mRNA expression values of iNOS, CFTR, and NHERF2 were extracted from the data set.

\section{Immunohistochemistry}

Paraffin-embedded human and mouse colon sections were stained for iNOS as follows. Certain colon sections from mouse were embedded in a swiss-roll configuration for better visualization. Slides were deparaffinized in xylene three times, 5 min each, followed by dehydration with ethanol. Antigen retrieval was done with Borg DeCloaker RTU (Biocare Medical, Concord, CA) in a pressure cooker for 5-10 min. Slides were cooled for $5 \mathrm{~min}$, and tissues were permeabilized with $0.2 \%$ Triton-X-100 in PBS for 10 min. Tissues were then blocked in $2.5 \%$ horse serum for $2 \mathrm{~h}$ or overnight. Slides were rinsed with $1 \mathrm{X}$ PBS and incubated with rabbit polyclonal iNOS (abcam, Cambridge, UK ; 1:100 to 1:150), rabbit polyclonal R3194 CFTR(1:100) and rabbit monoclonal NHERF2 (Cell signaling, Danvers, MA; 1:50) antibodies at $4{ }^{\circ} \mathrm{C}$ overnight. Slides were incubated with peroxidazed 1 (Biocare Medical, Concord, CA) to block endogenous peroxidase activity for $5 \mathrm{~min}$. Biotin-conjugated rabbit horseradish peroxidase antibody (Santa Cruz Biotechnology, Inc., Santa Cruz, CA) was used in 1:100 dilution for $30 \mathrm{~min}$, and Vectastain $\mathrm{ABC}$ reagent (Vector labs, Burlingame, CA) was used for signal amplification for another $30 \mathrm{~min}$. Slides were developed with 3-3'-diaminobenzidine solution (Biocare Medical, Concord, CA) according to the manufacturer's instructions. For histological examination, slides were lightly stained with hematoxylin and eosin. For immunofluorescence, polarized HT29-CL19A cells were fixed in 4\% formaldehyde and permeabilized with $0.2 \%$ Triton-X-100 in PBS for 10 min. Cells were blocked in 5\% BSA in PBS for $2 \mathrm{~h}$ and incubated with rabbit polyclonal R3194 CFTR (1:100) and mouse monoclonal iNOS (BD Biosciences, Franklin Lakes, NJ; 1:100 primary antibodies) at $4{ }^{\circ} \mathrm{C}$ overnight. Rabbit Alexa-fluor-488 and mouse Alexa-fluor-568 (Invitrogen, Carlsbad, CA) were used (1:100), cells were mounted, and images were taken with a Carl Zeiss confocal microscope (Thornwood, NY). The images were analyzed with ImageJ software (NIH; http://rsb.info.nih.gov/ij/). 


\section{Total Internal Reflection Fluorescence Microscopy}

Human colon tissue slides were immunostained for iNOS using rabbit polyclonal iNOS primary antibody from abcam and rabbit Alexa-fluor-488 secondary antibody. TIRF images were taken using inverted Olympus microscope with a $100 \mathrm{X}$ oil objective. Antibody coupled dye was excited at $488 \mathrm{~nm}$ and images of emitted fluorescence were captured at a resolution of $128 \times 128$ pixels $(1$ pixel $=0.33 \mu \mathrm{m})$ with EM-CCD camera.

\section{Proximity Ligation Assay}

Paraffin-embedded human colon sections were used for performing proximity ligation assay (Olink Bioscience, Uppsala, Sweden). Briefly, human colon sections were deparaffinized and incubated with mouse monoclonal iNOS (BD Biosciences; 1:100) and rabbit monoclonal NHERF2 (Cell Signaling; 1:50) primary antibodies at $4{ }^{\circ} \mathrm{C}$ overnight. Next day, PLA was performed with anti-rabbit (plus) and anti-mouse (minus) Duo link In Situ PLA probes on the tissue slides following manufacturer's instructions. Next day, images were taken using Zeiss LSM-5 Pascal confocal microscope.

\section{Measurement of CFTR-mediated I' Influx Using a YFP-based Halide Sensor}

HEK-293 cells with different protein expression profiles were co-transfected with pCDNA3-TM-Cl sensor. After $24 \mathrm{~h}$, the transfected cells were seeded in 12-24-well plates and cultured for another $24 \mathrm{~h}$. The cells were washed twice with $200 \mu 1136 \mathrm{mM}$ $\mathrm{NaNO}_{3}$ solution per well prepared in the wash buffer containing $137 \mathrm{mM} \mathrm{NaCl}, 4.5 \mathrm{mM}$ $\mathrm{KH}_{2} \mathrm{PO}_{4}, 1 \mathrm{mM} \mathrm{CaCl}_{2}, 1 \mathrm{mM} \mathrm{MgCl}, 10 \mathrm{mM}$ glucose, $5 \mathrm{mM}$ HEPES, pH 7.2 and $100 \mu \mathrm{l}$ of the same buffer was added to each well. Fluorescence readings were recorded at $37^{\circ} \mathrm{C}$ on a FLUOstar-Omega (BMG Labtech; Ortenberg, Germany) microplate reader equipped with HQ500/20X (500 $\pm 10 \mathrm{~nm})$ excitation and HQ535/30M (535 $\pm 15 \mathrm{~nm})$ emission filters and two syringe pumps. Intracellular YFP fluorescence was monitored continuously for 90-150 intervals 1 second each for each well. At $5 \mathrm{~s}$ after the start of fluorescence recording, $100 \mu \mathrm{l}$ of an influx buffer ( $272 \mathrm{mM} \mathrm{NaI}$ in the wash buffer) containing $250 \mu \mathrm{M}$ zaprinast was added to activate CFTR-mediated $\mathrm{I}^{-}$influx.

\section{Iodide Efflux Assay}

CFTR-mediated halide efflux was measured using an iodide efflux assay. For the induction of iNOS levels, the following cytokine composition (cytomix) was used: TNF$\alpha 10 \mathrm{U} / \mathrm{ml}, \mathrm{IFN}-\gamma 200 \mathrm{U} / \mathrm{ml}$, LPS $1 \mu \mathrm{g} / \mathrm{ml}$, IL-1 $\beta 1 \mu \mathrm{g} / \mathrm{ml}$. HT29-CL19A cells grown on 60

$\mathrm{mm}$ dishes were incubated with the cytomix for $48 \mathrm{~h}$. HT29-CL19A cells, which were not treated or treated with the cytomix, were subjected to iodide efflux assay as previously described (4). Briefly, cells were loaded for $60 \mathrm{~min}$ at room temperature with loading buffer (136 mM NaI prepared in the buffer with the following composition: $137 \mathrm{mM}$ $\mathrm{NaCl}, 4.5 \mathrm{mM} \mathrm{KH}_{2} \mathrm{PO}_{4}, 1 \mathrm{mM} \mathrm{CaCl} 2,1 \mathrm{mM} \mathrm{MgCl}$, $10 \mathrm{mM}$ glucose, $5 \mathrm{mM}$ HEPES, $\mathrm{pH}$ 
7.2). Extracellular NaI was washed away thoroughly (5-7 times) with efflux buffer (136 $\mathrm{mM} \mathrm{NaNO}$ prepared in the buffer as mentioned before replacing $136 \mathrm{mM} \mathrm{NaI}$ in the loading buffer) and then, cells were equilibrated for 1 min each for four times to establish a stable base line in efflux buffer alone. After establishing basal equilibrium with the nitrate buffer, cells were treated with $1 \mathrm{mM} \mathrm{L}$-Arginine for $15 \mathrm{~min}$ followed by zaprinast $(500 \mu \mathrm{M})$ to activate CFTR function. The iodide concentration of each aliquot collected at 1 min interval was determined using an iodide-sensitive electrode (Thermo Scientific, Waltham, MA) and converted to iodide content (nanomoles/min).

\section{Short-circuit Current Measurements}

Polarized colon epithelial cell (HT-29CL19A) monolayers were grown on Costar Transwell permeable supports (Cambridge, MA; filter diameter $6.5 \mathrm{~mm}$ ) until the monolayers reached confluency and a resistance of $\sim 1500 \mathrm{ohms}$. HT-29CL19A cells were pretreated with or without the cytomix with the composition as mentioned before for $48 \mathrm{~h}$ and the transwells were mounted in an Ussing chamber. Short-circuit currents were measured as described previously (5). Epithelial cells were bathed in Ringer's solution (mM) (Basolateral side: $140 \mathrm{NaCl}, 5 \mathrm{KCl}, 0.36 \mathrm{~K}_{2} \mathrm{HPO}_{4}, 0.44 \mathrm{KH}_{2} \mathrm{PO}_{4}, 1.3$ $\mathrm{CaCl}_{2}, 0.5 \mathrm{MgCl}_{2}, 4.2 \mathrm{NaHCO}_{3}, 10$ HEPES, 10 glucose, $\left.\mathrm{pH} 7.2,\left[\mathrm{Cl}^{-}\right]=149\right)$, and low $\mathrm{Cl}^{-}$ Ringer's solution (mM) (Apical side: $133.3 \mathrm{Na}$-gluconate, $5 \mathrm{~K}$-gluconate, $2.5 \mathrm{NaCl}, 0.36$ $\mathrm{K}_{2} \mathrm{HPO}_{4}, 0.44 \mathrm{KH}_{2} \mathrm{PO}_{4}, 5.7 \mathrm{CaCl}_{2}, 0.5 \mathrm{MgCl}_{2}, 4.2 \mathrm{NaHCO}_{3}, 10$ HEPES, 10 mannitol, $\mathrm{pH}$ $\left.7.2,\left[\mathrm{Cl}^{-}\right]=14.8\right)$ at $37^{\circ} \mathrm{C}$, and saturated with $95 \% \mathrm{O}_{2}$ and $5 \% \mathrm{CO}_{2}$. A $2 \mathrm{mV}$ pulse was applied every $1 \mathrm{~min}$ throughout the experiment to check for the integrity of the epithelia. After stabilization of basal $I_{s c}$ Cells were treated with $1 \mathrm{mM} \mathrm{L}$-Arginine for $15 \mathrm{~min}$ followed by adding zaprinast $(100-500 \mu \mathrm{M})$ to the basolateral side. $\mathrm{CFTR}_{\text {inh }}-172(20-50$ $\mu \mathrm{M}$ ) was added to the apical side at the end of the experiment to verify that the shortcircuit currents were CFTR-dependent.

\section{Ratiometric Fluorescence Resonance Energy Transfer Microscopy and Data Analysis}

For ratiometric FRET, HEK-293 cells were grown on glass-bottom dishes (MatTek, Ashland, MA) and transfected with pcDNA3-empty vector, pcDNA3-iNOS, and pcDNA3-m-cygnet 2.1 using Lipofectamine 2000. Cells were washed twice with Hank's balanced salt solution (HBSS) and mounted on an inverted Olympus wide-field microscope (IX51, U-Plan Fluorite 60x 1.25 NA oil-immersion objective) for FRET imaging. The stage was maintained at $37^{\circ} \mathrm{C}$ constantly, and the cells were maintained in the dark in HBSS. After establishing the baseline, zaprinast was added as indicated. Ratiometric FRET imaging was performed as described previously (5). Time-lapse images were obtained with $1 \mathrm{x} 1$ binning, 100-300 ms exposure time and 1 min intervals. Images were acquired using an EM CCD camera (Hamamatsu) mounted on an Olympus IX51. The light source used was 300W xenon lamp with a neutral density filter. A JP4 CFP/YFP filter set was used for image capture (Chroma, Brattleboro, VT) which includes a 430/25 $\mathrm{nm}$ excitation filter, a double dichroic beam splitter and two emission filters 
(470/30 nm for CFP and 535/30 nm for FRET emission) alternated by filter-change controller Lambda 10-3 (Sutter instruments, Novato, CA). Slidebook 4.2 software was used for data analysis. Following background subtraction, multiple regions of interest $(10-20)$ were selected (3-5 cells) for data analysis using ratio module. The emission ratio (CFP/FRET) was obtained from CFP and FRET emission of background subtracted cells.

\section{Co-immunoprecipitation in Colon Tissue}

To immunoprecipitate CFTR and NHERF2 from DSS-treated mouse colon, rabbit polyclonal iNOS antibody $(1 \mu \mathrm{g})$ was mixed with $20 \mu \mathrm{l}$ protein $\mathrm{A} / \mathrm{G}$ agarose for $1 \mathrm{~h}$ at $4^{\circ} \mathrm{C}$. Rabbit polyclonal non-immune IgG $(1 \mu \mathrm{g})$ was used as control. Bound IgG was washed once with $1 \mathrm{ml} 0.1 \mathrm{M}$ N-ethyl morpholin (NEM; pH 7.5) buffer. The IgG is cross linked to protein $\mathrm{A} / \mathrm{G}$ agarose using $10 \mathrm{mM}$ Dimethyl pimelimidate (DMP; Pierce Chem. Co. Dallas, TX) in NEM buffer for $30 \mathrm{~min}$ at $22^{\circ} \mathrm{C}$. The cross-linking reaction is stopped by washing the beads once with $100 \mathrm{mM}$ Tris-base ( $\mathrm{pH} 8.0)$. The cross-lined beads were incubated with the lysate $(1 \mathrm{mg})$ in $1 \mathrm{x}$ PBS, containing $0.2 \%$ Triton X-100 overnight at $4^{\circ} \mathrm{C}$. Next day, proteins were eluted using glycine buffer $\mathrm{pH}$ 2.2. Proteins were subjected to SDS-PAGE and probed with the respective antibodies.

\section{Real-time PCR}

Total mRNA was isolated from HT-29CL19A cells (untreated or pretreated with cytomix) using RNeasy kit from Qiagen (Hilden, Germany). The RNA was treated with DNase I at $37^{\circ} \mathrm{C}$ for $1 \mathrm{~h}$, and the concentration of each sample was measured using NanoDrop (Thermo Scientific, Waltham, MA). DNA-free RNA (1 $\mu \mathrm{g})$ was converted to cDNA by using Transcriptor first-strand cDNA synthesis kit (Roche, Basel, SUI) according to the manufacturer's instructions. Real-time PCR reaction was performed using TaqMan ${ }^{\circledR}$ probe based method in the LightCycler 480 instrument (Roche, Basel, SUI). Probes and primers were designed using universal probe library (Roche). The target genes were normalized with the TBP gene (probe 3). The following probes and left and right primers were used for real-time PCR amplification of each of the genes:

iNOS (probe 37)

Left: ACCAGTACGTTTGGCAATGG

Right: TCAGCATGAAGAGCGATTTCT

CFTR (probe 8)

Left: CACAGCAACTCAAACAACTGG

Right: TGTAACAAGATGAGTGAAAATT

NHERF2 (probe 24) 


\section{Left: TCCGAAGCTGGCAAGAAG \\ Right: ATAGCCCTGAGGTCCCTTTC}

\section{cGMP Assay}

Mice colons were quickly frozen in liquid nitrogen and ground to a fine powder. Tissue was dissolved in $600 \mu \mathrm{l}$ of $6 \%$ trichloroacetic acid (TCA) and spun at 10,000 rpm at $4^{\circ} \mathrm{C}$. Pellet was lysed in lysis buffer (Tris-Cl EDTA containing $0.5 \%$ Triton-X-100 and $0.1 \%$ SDS) and the supernatant was used for protein quantitation and further processing. Supernatant was then transferred to a glass vial and washed three times with water saturated ether (1:1) with water forming the top phase. Sample of interest exists in the water phase and is taken out from the top after each wash. Residual ether was removed by boiling the samples briefly at $68^{\circ} \mathrm{C}$. Samples were used for cGMP measurement by direct cGMP ELISA from Enzo Life Sciences (Farmingdale, NY) as per manufacturer's

directions. A cGMP standard provided in the kit was run in parallel for the quantitation of cGMP in the samples.

\section{NO Assay}

Colon tissue was homogenized in $1 \mathrm{ml}$ of $1 \mathrm{X}$ PBS $0.2 \%$ Triton-X-100 buffer and protein concentration of the supernatant was determined. $100 \mu \mathrm{l}$ of the tissue supernatant was mixed with equal volume of Griess reagent and incubated for $20 \mathrm{~min}$. Plate was read at $540 \mathrm{~nm}$ and the nitrite levels were determined using a sodium nitrite standard curve. Nitrite levels were normalized with the total protein concentration. 


\section{CHAPTER 4. RESULTS}

\section{Purification of Full-length and Truncated iNOS Proteins}

In this part of the study, affinity epitope-tagged full-length iNOS proteins involving Flag and HA tags at the N-terminal were first cloned into pcDNA3 vector. These constructs were primarily used for protein purification, performing biochemical protein-protein interaction assays like pull-down assays and co-immunoprecipitation. Also, Flag- and HA-iNOS were cloned into pLenti destination vector. pLenti-Flag- and HA-iNOS were later used for making stable cell lines after initial selection on $6 \mu \mathrm{g} / \mathrm{ml}$ puromycin for 1 week till only positive cells survived and later, trypsinized and maintained on $2 \mu \mathrm{g} / \mathrm{ml}$ puromycin containing DMEM F-12 media. Five to six $100 \mathrm{~mm}$ dishes of cells expressing Flag- or HA-iNOS were maintained till complete confluence. The cells were then washed with 1 XPBS twice and lysed with PBS containing $0.2 \%$ Triton-X-100 and protease inhibitor cocktail. The cell lysate was mixed on a shaker for $10 \mathrm{~min}$ at $4{ }^{\circ} \mathrm{C}$ and centrifuged for $10 \mathrm{~min}$ at $13,000 \mathrm{rpm}$. The supernatant containing total protein was immunoprecipitated using $\alpha$-Flag, $\alpha$-HA beads overnight. The beads with bound protein were washed and the protein was eluted with $100 \mathrm{mM}$ glycine ( $\mathrm{pH}$ 2.2) and quickly neutralized with $150 \mathrm{mM}$ tris ( $\mathrm{pH} \mathrm{8.8).} \mathrm{The} \mathrm{eluted} \mathrm{protein} \mathrm{was} \mathrm{subjected} \mathrm{to} \mathrm{SDS-}$ PAGE on $4-15 \%$ gel (Bio-Rad, Hercules, CA) and visualized with Gel Code Blue. A coomassie gel with HA-iNOS is shown in Figure 4-1.

iNOS-C-tail (1041-1153 amino acids) retaining the PDZ-motif was cloned into pET-41- and pTriEx-4 vectors to generate GST-His-S-iNOS-C-tail and His-S-iNOS-Ctail, respectively. The idea of generating this construct was increased options of affinity tags, ease of purification with a smaller construct and performing biochemical assays. GST stands for Glutathione-S-Transferase protein $(26 \mathrm{KDa})$ which is a commonly used epitope tag for protein purification and exhibits high affinity for glutathione agarose Proteins were induced in an E.coli bacterial system using IPTG and retrieved after bacterial lysis using suitable lysis buffers as described in Chapter 3 Methods. The GSTfusion and His-tagged proteins were purified using affinity chromatography involving glutathione beads and talon beads, respectively. Talon beads are precharged with $\mathrm{Co}^{2+}$ with a unique tetradentate metal chelator and exhibit high specificity for His-tagged proteins. For the GST-tagged protein elution was done using $10 \mathrm{mM}$ glutathione in PBS ( $\mathrm{pH}$ 7.4). His-S-iNOS-C-tail protein was eluted in $200 \mathrm{mM}$ Imidazole prepared in resuspension buffer (described in Chapter 3 Methods). Glutathione and imidazole act competitive to GST and Histidine residues binding to the respective resins facilitating the elution of the proteins. The eluted proteins were further purified using size exclusion chromatography. Purified GST-His-S-iNOS-C-tail and His-S-iNOS-C-tail are shown in Figures 4-2A and 4-2B respectively, on a coomassie stained gel. The protein smear appearing below the major band in Figure 4-2B represents partial degradation of the protein.

Alternatively, fluorescent CFP-tagged iNOS and Yellow (a variant of YFP)tagged NHERF2 constructs were obtained or cloned to perform protein-protein 


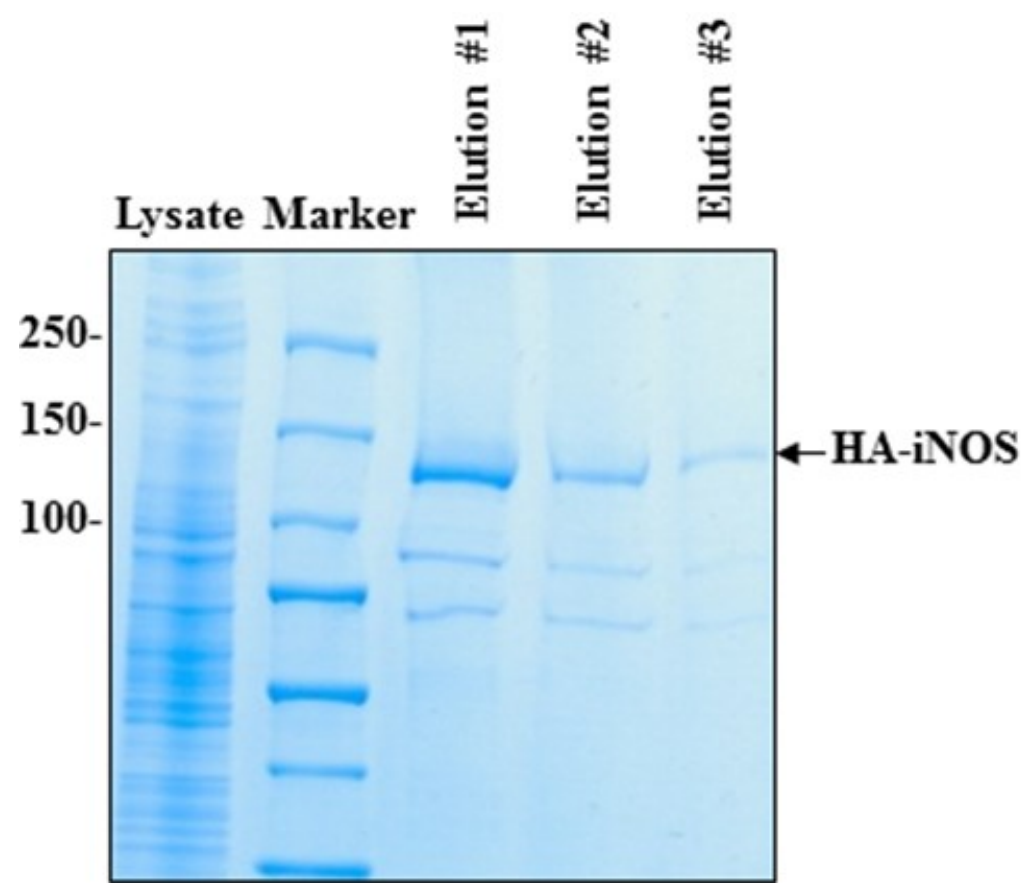

Figure 4-1. Purification of HA-iNOS

Coomassie stained gel showing purified full-length HA-iNOS $(\sim 130 \mathrm{KDa})$ from HAiNOS overexpressing HEK-293 cells. 
A

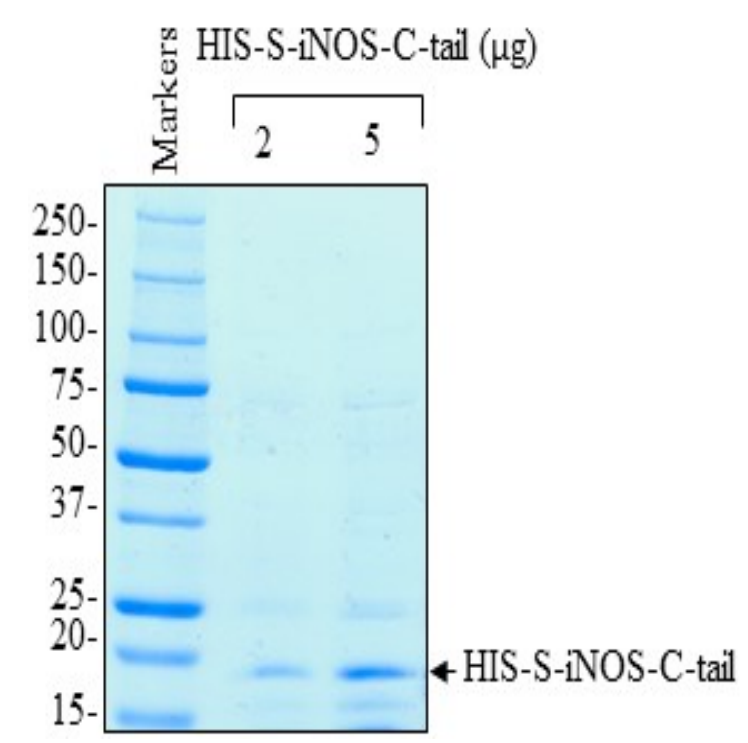

B

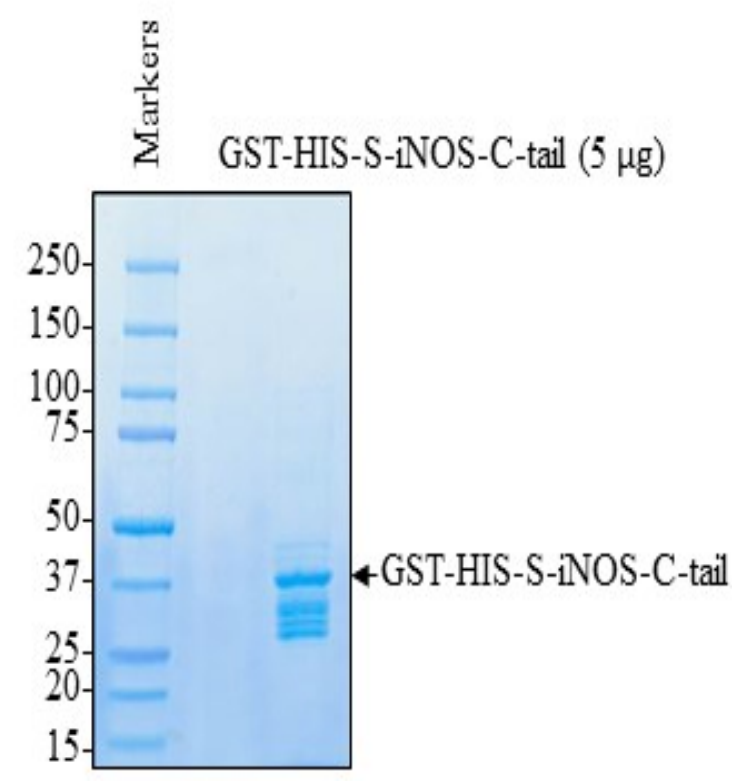

\section{Figure 4-2. Purification of truncated iNOS proteins}

Coomassie stained gel showing purified (A) His-S-iNOS-C-tail and (B) GST-His-SiNOS-C-tail (1041-1153 amino acids) expressed in E.coli. 
interaction studies in live cells using FRET. Yellow tag was preferred over YFP because of its improved spectral properties and better signal. HEK-293 cells were the ideal choice to perform protein-protein interaction studies and make stable cell lines because they originate from human, are easy to transfect, and respond to various agonists.

\section{iNOS Exhibits High Affinity Interaction with NHERF2}

Given that iNOS harbors a putative PDZ motif to bind to PDZ-domain containing scaffolding proteins, we first screened the interaction of iNOS with different PDZ proteins to identify the high-affinity interactions of iNOS. An array of GST-tagged PDZ proteins (NHERF1, NHERF2, and PDZK1) referred to as a PDZ array was generated. A PDZ array comprises of proteins immobilized on nitrocellulose membrane using a slot blot applicator. It allows screening of preferential protein-protein interactions and the relative binding affinities of various PDZ-proteins towards a specific protein at the same time. In the mentioned experiment, the immobilized PDZ-proteins were screened for binding to GST-His-S-iNOS-C-tail (100 ng) in buffer comprised of $0.1 \%$ Tween-20 and $2 \%$ BSA in PBS in an overnight binding at $4^{\circ} \mathrm{C}$. Our results demonstrated that NHERF2 showed the highest binding affinity towards iNOS, whereas NHERF1 showed a weak binding and PDZK1 did not bind to iNOS (Figure 4-3A). A similar assay is referred to as a dot-blot assay where protein samples are dropped on to a nitrocellulose membrane in a volume of 1-2 $\mu 1$ using a pipette on a self-drawn grid and allowed to dry. His-tagged protein His-S-MAST205 was used as a positive control for the assay. The second interacting protein GST-His-S-iNOS-C-tail (100 ng) is added to the membrane in 3-5 ml of $0.1 \%$ Tween- 20 and $2 \%$ BSA in PBS and binding is performed overnight at $4{ }^{\circ} \mathrm{C}$. Next day the membrane is washed six times with $0.1 \%$ Tween- 20 in TBS and probed with SHRP antibody (1:5000) at room temperature for $1 \mathrm{~h}$. The membrane is again washed extensively with $0.1 \%$ Tween-20 in TBS six times for five min each. The membrane is developed with ECL ${ }^{\mathrm{TM}}$ chemiluminescence solution. The data is shown in Figure 4-3B.

Given the fact that iNOS is not normally expressed under native conditions, HEK293 cells were transiently transfected with full-length pcDNA3-iNOS $(1 \mu \mathrm{g})$ using Lipofectamine 2000. Cells are subsequently lysed in $0.2 \%$ Triton-X-100 buffer in PBS. The rationale behind using Triton-X-100 based lysis buffer is that Triton-X-100 is a nonionic detergent which does not interfere with protein-protein interactions, After the lysis, lysate enriched in proteins is subjected to a pull-down assay with GST-NHERF2. The rationale of this experiment is to elucidate interaction of NHERF2 with full-length iNOS. In this assay, GST-NHERF2 $(0-15 \mu \mathrm{g})$ is allowed to mix with the HEK-293 iNOS overexpressing cell lysate for $1 \mathrm{~h}$. GST-NHERF2 interacting protein complexes are then immobilized on glutathione beads overnight. Next day, beads are washed to remove unbound and non-specific proteins and the protein complexes are eluted in 5X laemmli buffer containing 5\% $\beta$-mercaptoethanol, subjected to SDS-PAGE and western blot. The blot was probed with a mouse monoclonal against iNOS. A concentration-dependent interaction was observed between iNOS and GST-NHERF2 (Figure 4-4). Coimmunoprecipitation and immunoblotting were also used to detect the interaction between full-length iNOS and NHERF2 in an overexpression condition. These 
A

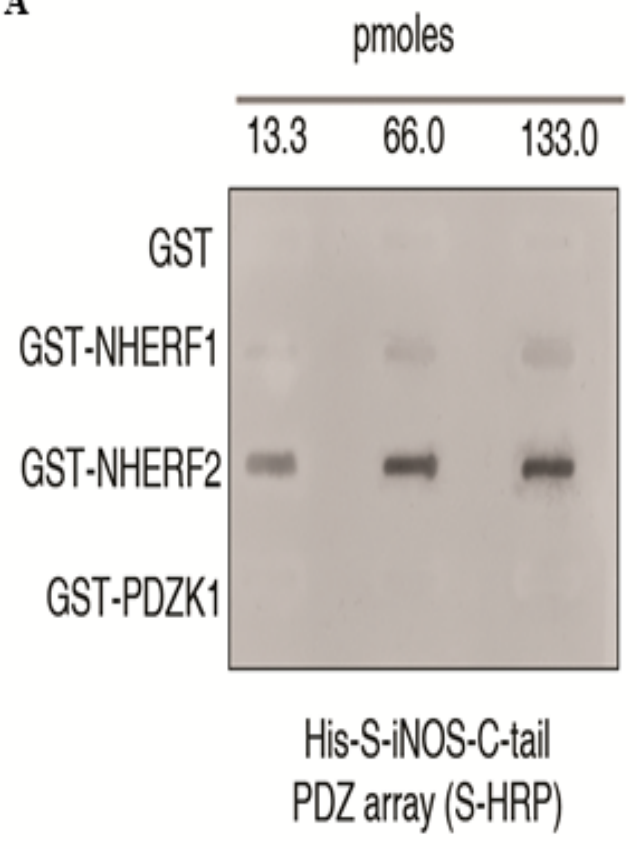

B

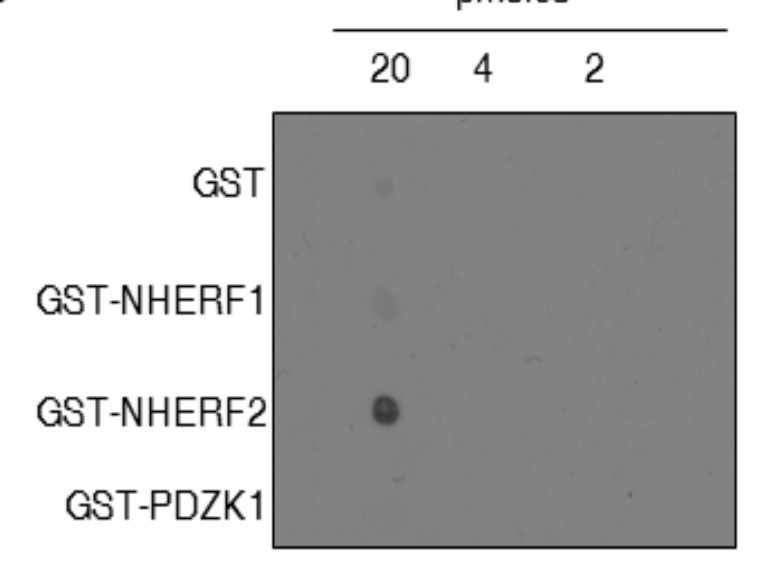

His-S-MAST205

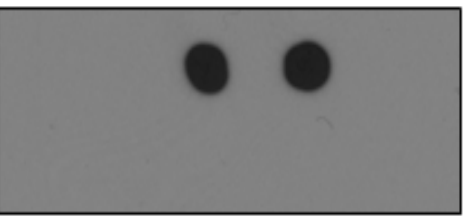

His-S-iNOS-C-tail

Dot-blot assay (S-HRP)

\section{Figure 4-3. iNOS preferentially binds NHERF2 among NHERF proteins}

An immunoblot showing GST-tagged PDZ proteins immobilized on nitrocellulose membrane in (A) a slot-blot (B) dot-blot format probed with S-HRP for detecting interaction with His-S-iNOS-C-tail. 


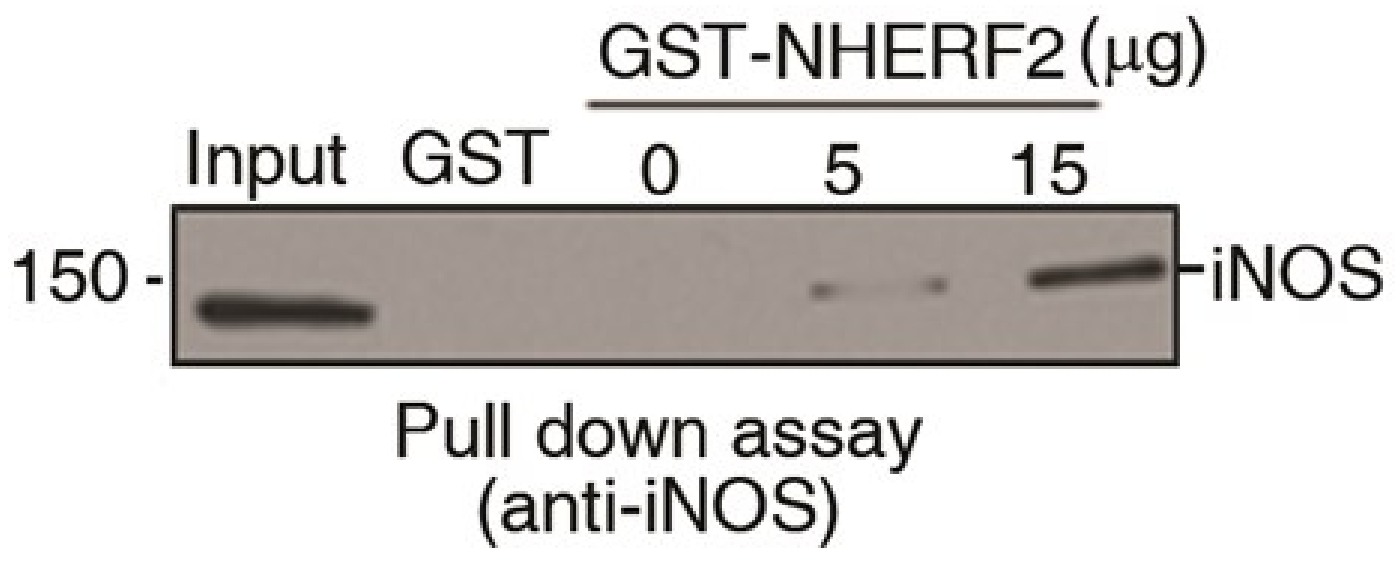

Figure 4-4. NHERF2 immunoprecipitates full-length iNOS from the cells

An immunoblot for full-length iNOS, overexpressed in HEK-293 cells immunoprecipitated with GST-NHERF2. 
procedures are based on the principle of immunoprecipitation which in coimmunoprecipitation principally involves capturing native interactions in the cells using antibodies conjugated to resins. We generated a stable HA-iNOS (and Flag-iNOS) overexpressing HEK-293 cell line using a lentiviral expression based system and the full length purified protein is shown in Figure 4-1. Certain protein-protein interactions can be quite transient and unstable and can disappear in stressful lysis and wash steps. For transient interactions, proteins can be covalently cross-linked using a cross-linker, which would help prevent loss of binding partners. NHERF2 interaction with iNOS could not be detected as such and required cross-linker. We used DSP for cross-linking the proteins. A temperature condition of $37^{\circ} \mathrm{C}$ and preciseness of time is very critical for successful cross-linking. DSP $(10 \mathrm{mM})$ was added to the cells in pre-warmed PBS with $\mathrm{Ca}^{2+}(100$ $\mu \mathrm{M})$ and $\mathrm{Mg}^{2+}(1 \mathrm{mM})$ and incubated for $10 \mathrm{~min}$ at $37^{\circ} \mathrm{C}$ followed by lysis in cold PBS $0.2 \%$ Triton-X-100 buffer. HA beads $(100 \mu 1)$ were added to immunoprecipitate the complex. The next day, samples were eluted using glycine buffer and western blot was performed and probed with rabbit polyclonal NHERF2 antibody. We detected that NHERF2 co-immunoprecipitated with iNOS upon cross-linking the complex (Figure 4-5).

Next, using direct sensitized FRET, we investigated whether the interaction could be detected in live cells. FRET is a procedure to detect protein-protein interactions in live cells involving a radiationless mode of energy transfer from one chromophore referred to as donor to second chromophore referred to as acceptor that exists within a diameter of $100 \mathrm{~nm}$ of the donor. HEK-293 cells were co-transfected with CFP-iNOS (donor) and Yellow-NHERF2 (acceptor) to measure normalized corrected FRET value (N-FRETc). The FRET is being mentioned as corrected as the bleed-through values deriving from acceptor excitation in the absence of donor with the donor excitation wavelength have been accounted for in the final measurement. Using the FRET module of SlideBook 4.2 software, an approximate FRETc value of $35 \pm 4 \% \mathrm{~N}$ - was estimated at the plasma membrane in cells that co-expressed CFP-iNOS and Yellow-NHERF2 (Figure 4-6A). The control group comprised of HEK-293 cells co-expressing CFP-iNOS donor with Yellow-fluorophore acceptor. Little or no FRET signal was observed in the controls (Figure 4-6B).

The specificity of PDZ-dependent iNOS-NHERF2 interaction was further investigated using a highly sensitive amplified luminescent proximity homogenous assay $\left(\right.$ AlphaScreen $\left.{ }^{\mathrm{TM}}\right)$. The assay is referred as homogenous as no washing steps are involved in the process. Also, AlphaScreen ${ }^{\mathrm{TM}}$ can detect very low affinity interactions in the picomolar range. The fundamental requirement of an AlphaScreen ${ }^{\mathrm{TM}}$ readout is the proximity of excited donor and acceptor beads within $200 \mathrm{~nm}$. Donor and acceptor beads are conjugated with affinity molecules that can be matched with the epitopes on target proteins. Donor beads contain a photosensitizer, phthalocyanine, which when excited at $680 \mathrm{~nm}$ generates an excited and a reactive form of $\mathrm{O}_{2}$, singlet oxygen. Singlet oxygen would transfer energy to thioxene derivatives within the acceptor bead if present in the right proximity of the donor bead leading to an emission of 520-620 nm wavelength which is quantified as counts per second (cps) in a microplate reader. The usual readout of a pair-wise protein-protein interaction with AlphaScreen ${ }^{\mathrm{TM}}$ is in the form of a bell- 


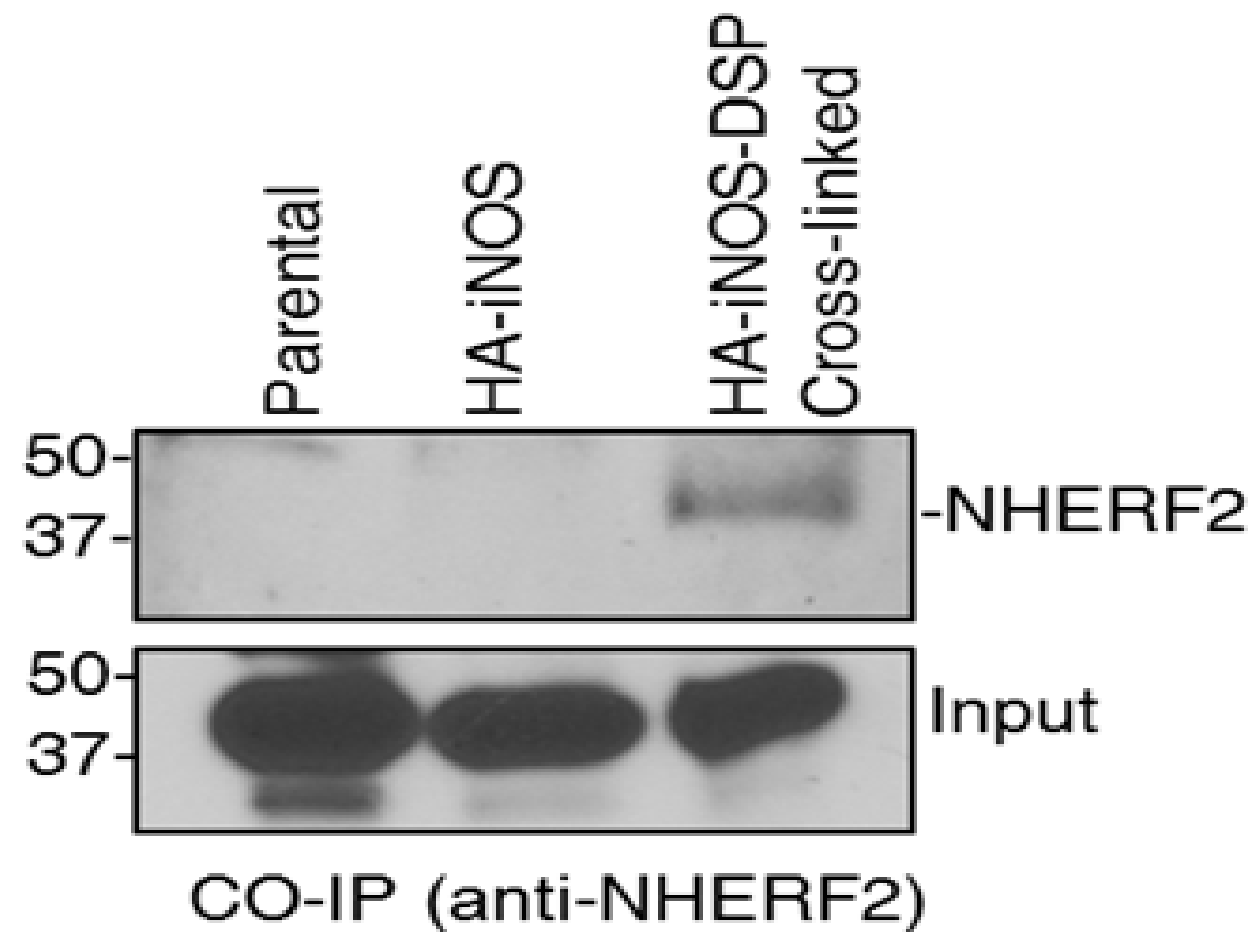

Figure 4-5. iNOS and NHERF2 exist within a protein complex in the cells

Western-blot data to depict co-immunoprecipitated NHERF2 and HA-iNOS in HEK-293 cells using a cross-linker DSP and probed with rabbit NHERF2 polyclonal antibody. 


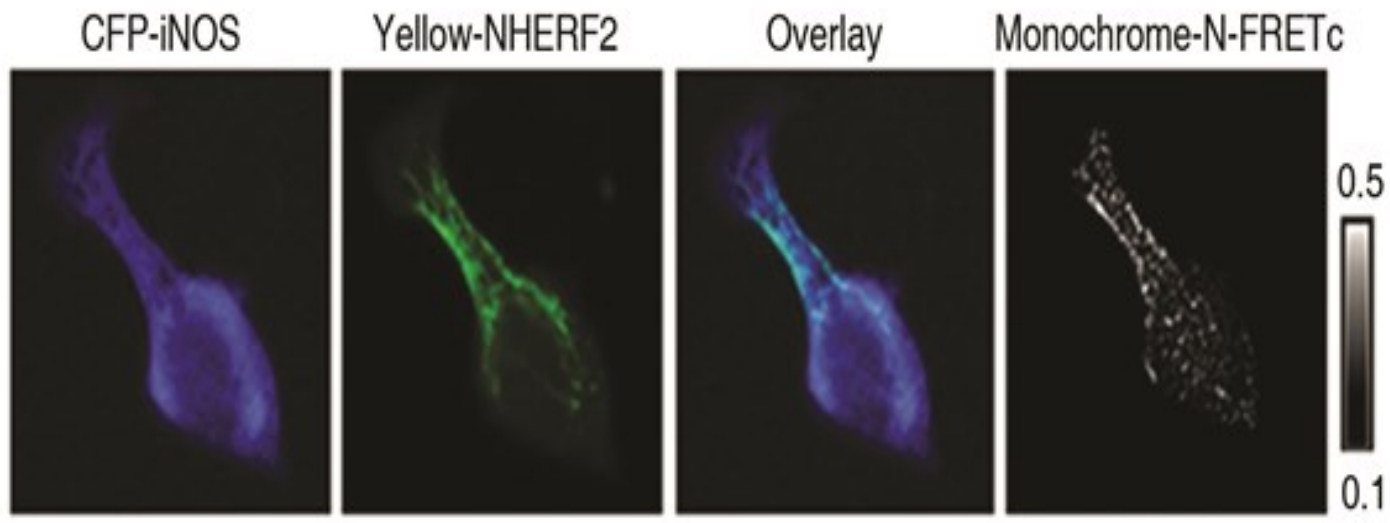

B

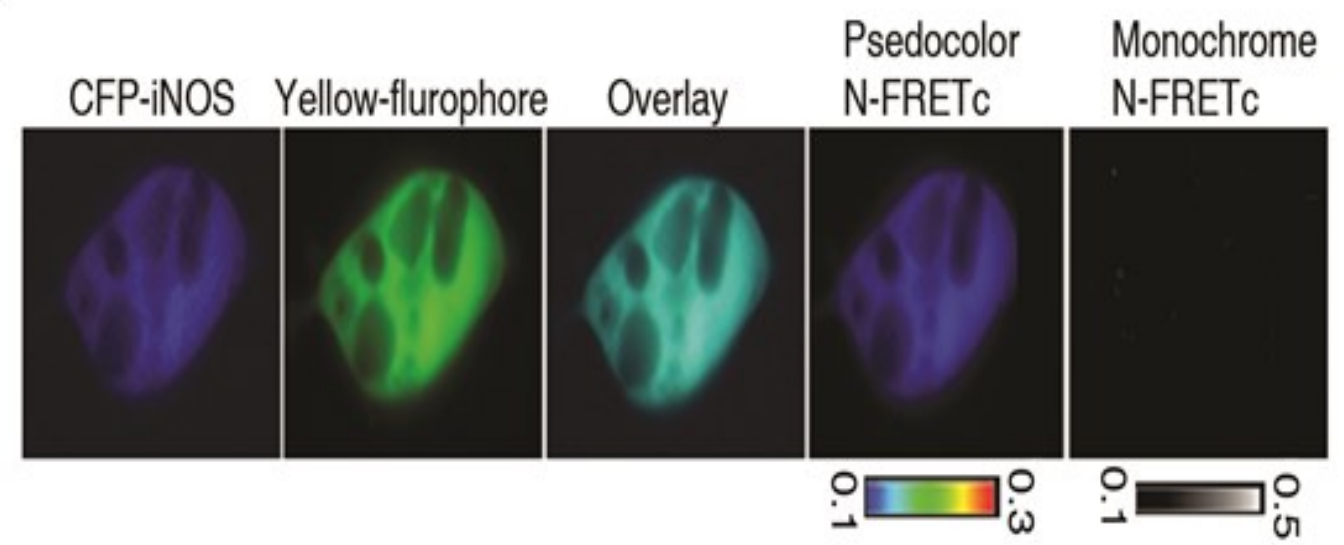

Figure 4-6. iNOS and NHERF2 protein complex exists at the plasma membrane in the cells

(A) Representative pseudocolor images showing the expression of CFP-iNOS and Yellow-NHERF2 in HEK-293 cells. The intensity of direct sensitized FRET (N-FRETc) between CFP-iNOS and Yellow-NHERF2 at the plasma membrane was shown in a monochrome mode, according to a temperature-based look-up table ( $\mathrm{n}=3$ independent experiments). (B) Representative pseudocolor images showing the expression of CFPiNOS and Yellow-fluorophore in HEK-293 cells. N-FRETc in the co-transfected cells was shown in pseudocolor and monochrome modes. 
shaped curve termed as Hook-effect. Hook-effect is the common read-out profile of saturable bimolecular detection systems including FRET and AlphaScreen ${ }^{\text {TM }}$. Standard curves obtained with AlphaScreen ${ }^{\mathrm{TM}}$ are characterized by a titratable increase in signal until it attains a maximal value termed as hook point. After the hook point signal begins to drop as excessive concentrations of target molecules oversaturates the donor and the acceptor beads and restricts their association. The interaction between GST-NHERF2 $(100 \mathrm{nM})$ and GST-His-S-iNOS-C-tail $(30 \mathrm{nM}$ to $300 \mu \mathrm{M})$ or Biotin-iNOS-C-tail peptide (last 10 amino acids; QPSSLEMSAL; $30 \mathrm{nM}$ to $300 \mu \mathrm{M}$ ) was studied using AlphaScreen ${ }^{\mathrm{TM}}$. GST $(100 \mathrm{nM})$ was used as a control in these experiments. A micromolar range interaction was observed for GST-His-S-iNOS-C-tail and biotin-iNOS peptide with GST-NHERF2, while no interaction was observed in the GST control (Figures 4-7A and 4-7B). The specificity of the interaction was demonstrated by the competition-based assay between biotin-iNOS and iNOS peptides for interaction with GST-NHERF2. The interaction between GST-NHERF2 and biotin-iNOS peptide was disrupted in a concentration-dependent manner by the untagged iNOS peptide, while DMSO did not disrupt the interaction (Figure 4-8).

Taken together, our data demonstrate that iNOS exhibits a high-affinity interaction with NHERF2. PDZ proteins have been shown to mediate the localization of various proteins at or near the plasma membrane, thereby enabling cell polarization to mediate vectorial functions (120). Given that, NHERF2 binds to PDZ-domain proteins predominantly associating with the plasma membrane and there is high amount of FRET signals between iNOS and NHERF2 at the plasma membrane, we reasoned that iNOSNHERF2 interaction may play an important role in mediating apical localization of iNOS and in coupling it with other apical membrane-bound proteins. Towards this end HEK293 cells with a knockdown of NHERF2 were generated and about 50\% knockdown was achieved with these cells compared to HEK-293 cells with scrambled siRNA (Figure 4-9). These cells were immunostained for overexpressed iNOS to demonstrate the role of NHERF2 in regulating sub-cellular localization of iNOS. HEK-293 cells with scrambled siRNA exhibited higher sub-apical membrane localization compared to NHERF2 knockdown cells (Figure 4-10). In order to demonstrate the relevance to colon epithelial cells, human colon epithelial cell line HT29-CL19A were transfected with iNOS and iNOS- $\triangle$ PDZ, with the latter lacking the ability to bind NHERF2 (Figure 4-11). First, HEK-293 cells were transfected with iNOS and iNOS- $\triangle \mathrm{PDZ}$ and lysed, and a pull-down was performed with GST-NHERF2 immobilized on glutathione beads in a similar manner as described above. Interaction of iNOS with GST-NHERF2 served as positive control for the experiment. Based on this data, HT-29CL19A cells were immunostained for iNOS and it could be demonstrated that removal of PDZ-motif reduced sub-apical localization of iNOS (Figure 4-12). This data implies that iNOS-NHERF2 interaction has pathological relevance.

\section{NHERF2 Bridges CFTR and iNOS into a Macromolecular Signaling Complex}

Given that (i) iNOS binds with high affinity to NHERF2 and localizes primarily at the membrane (108); and CFTR interacts with NHERF2 with high affinity (42), we 
$\mathbf{A}$

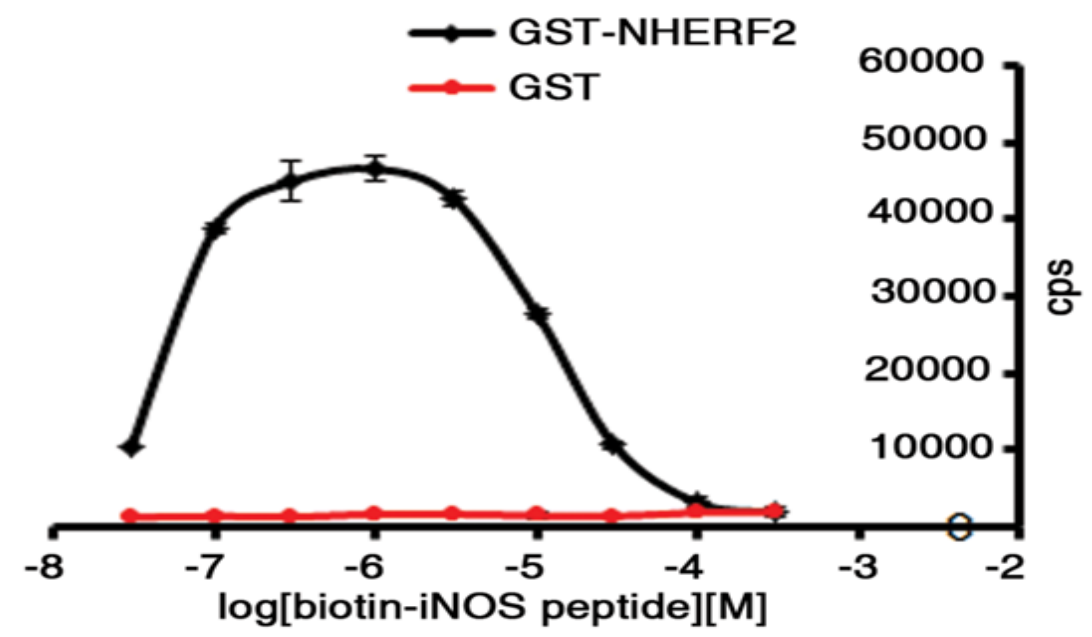

B

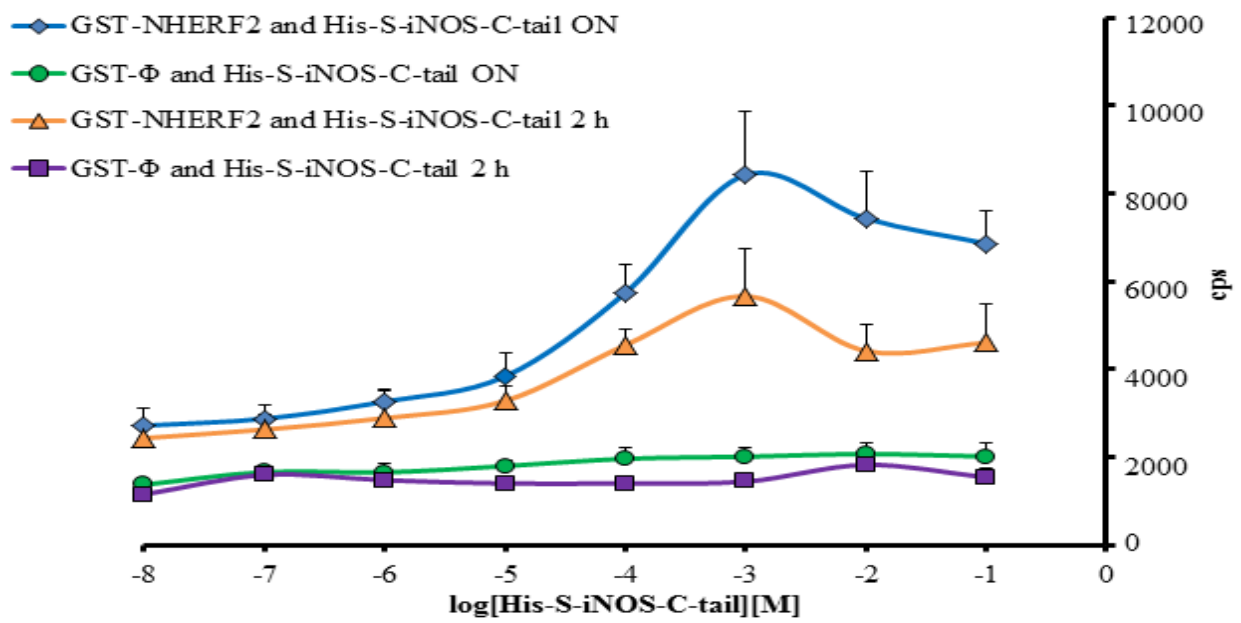

Figure 4-7. GST-NHERF2 and iNOS protein fragments interaction using AlphaScreen $^{\text {TM }}$ assay

AlphaScreen ${ }^{\mathrm{TM}}$ assay to determine the binding affinity between (A) biotin-iNOS-C-tail peptide and GST-NHERF2 and (B) His-S-iNOS-C-tail and GST-NHERF2 ( $n=3$ samples). 


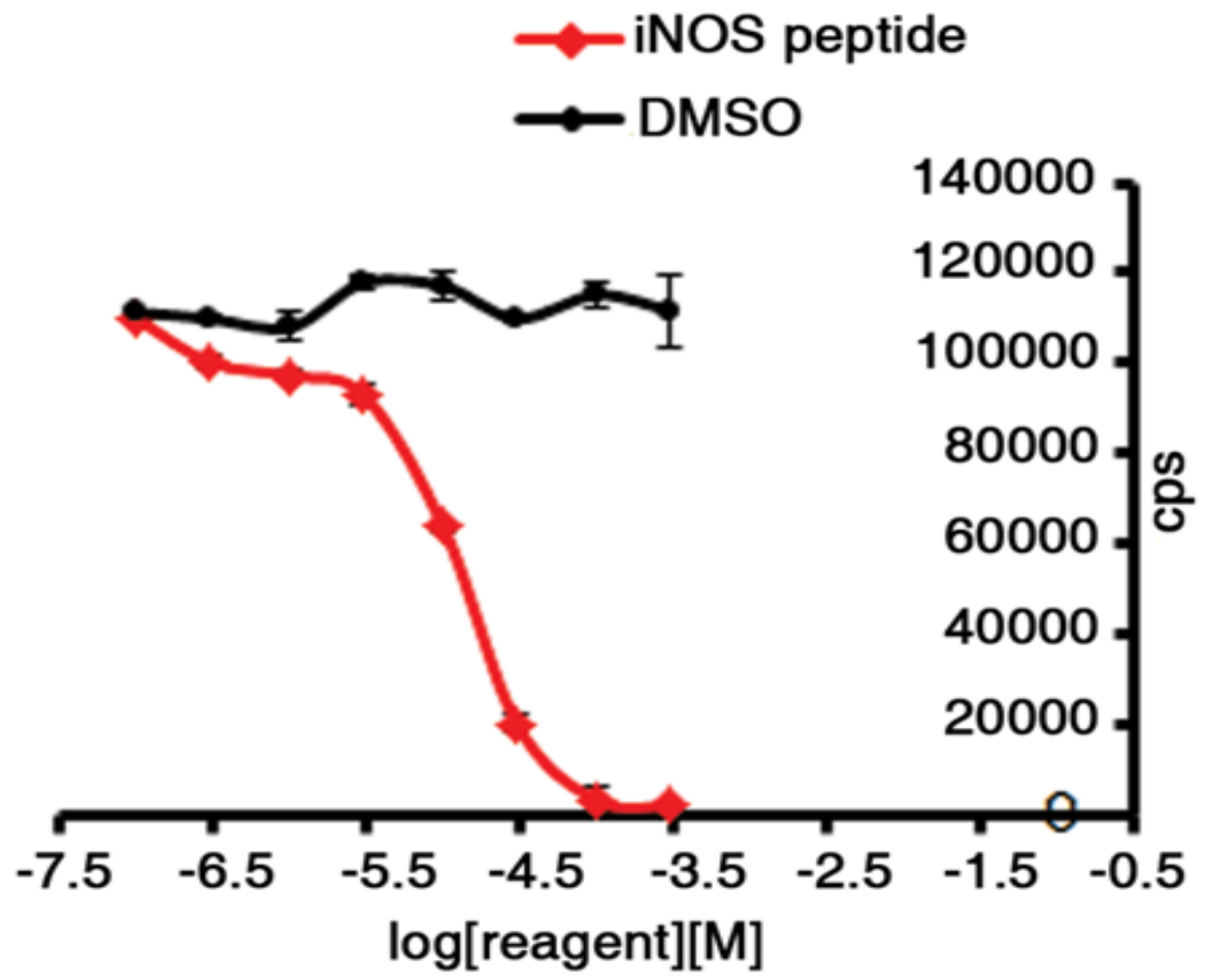

Figure 4-8. AlphaScreen ${ }^{\mathrm{TM}}$ competition assay for GST-NHERF2 and iNOS-C-tail peptide

AlphaScreen ${ }^{\mathrm{TM}}$ competition assay shows that a non-biotinylated iNOS peptide competes against biotin iNOS peptide for binding to GST-NHERF2 protein, verifying the specificity of the interaction ( $\mathrm{n}=3$ samples). 

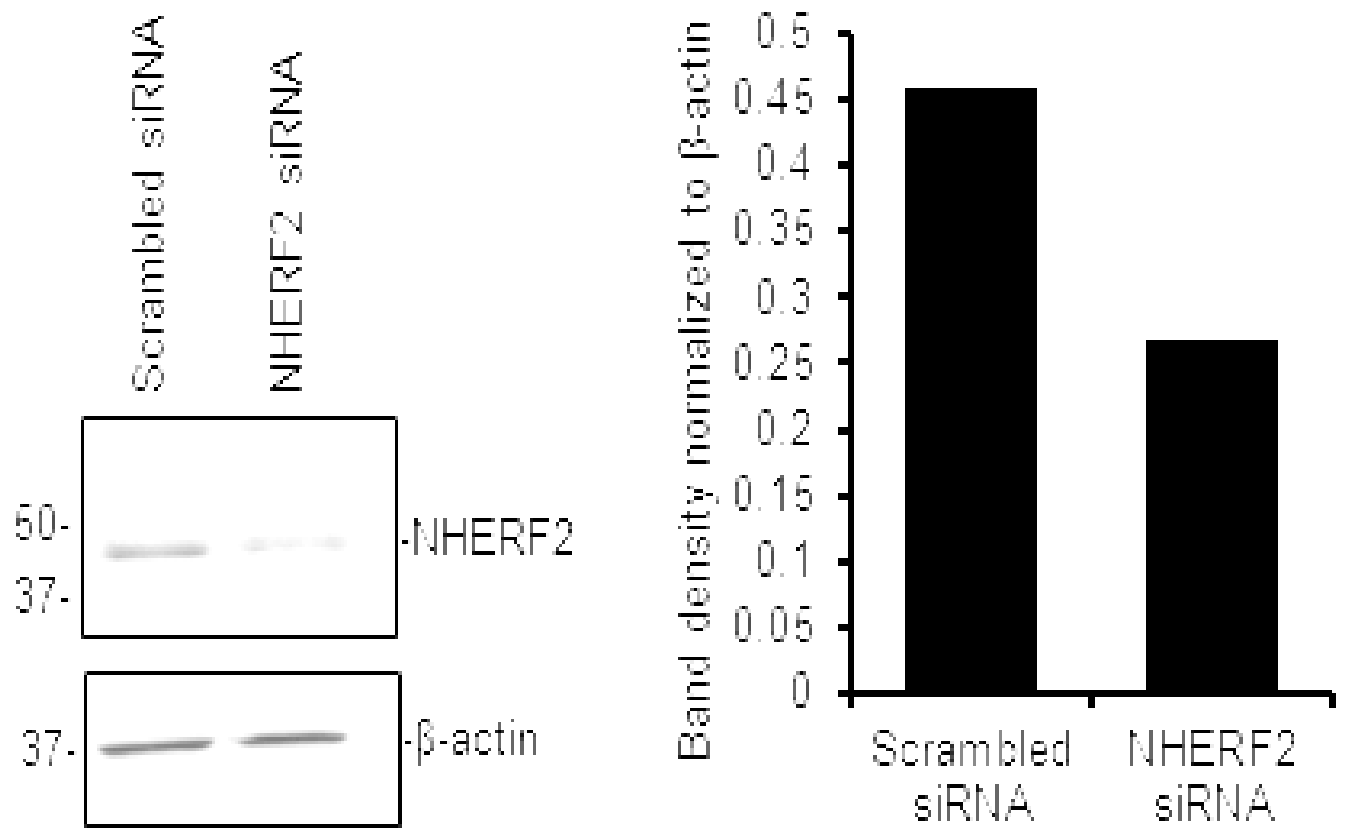

Figure 4-9. NHERF2 knockdown in HEK-293 cells

Western blot to demonstrate NHERF2 knockdown in HEK-293 cells using lentiviral siRNA particles. Bar-graph to quantitate around 50\% knockdown of NHERF2 compared to scrambled siRNA. $\beta$-actin was used for protein normalization. 


\section{HEK-293 Scrambled siRNA (Parental)}

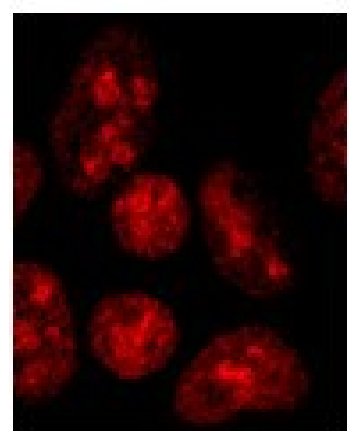

HEK-293 Scrambled siRNA (iNOS)
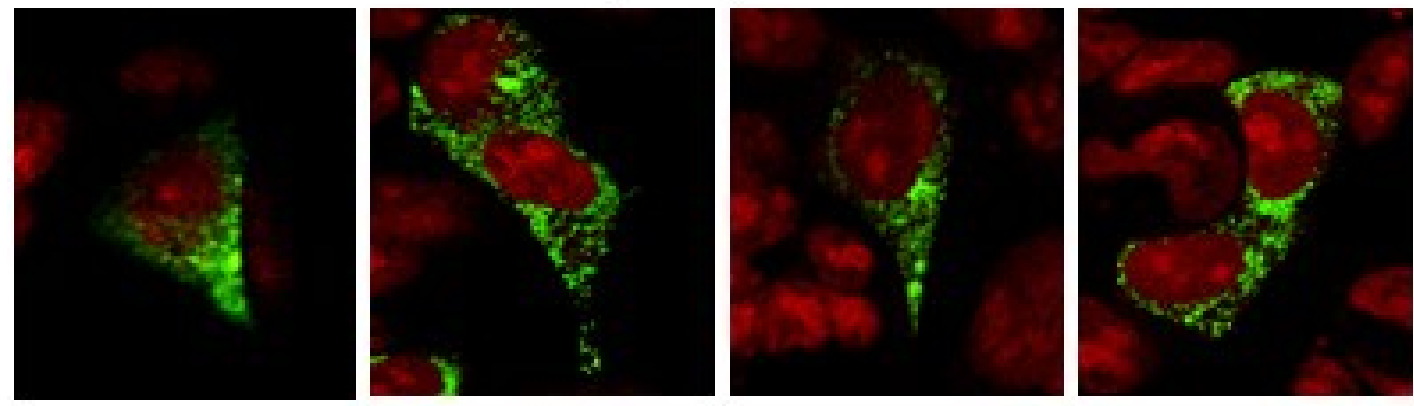

\section{HEK-293 NHERF2 siRNA (iNOS)}
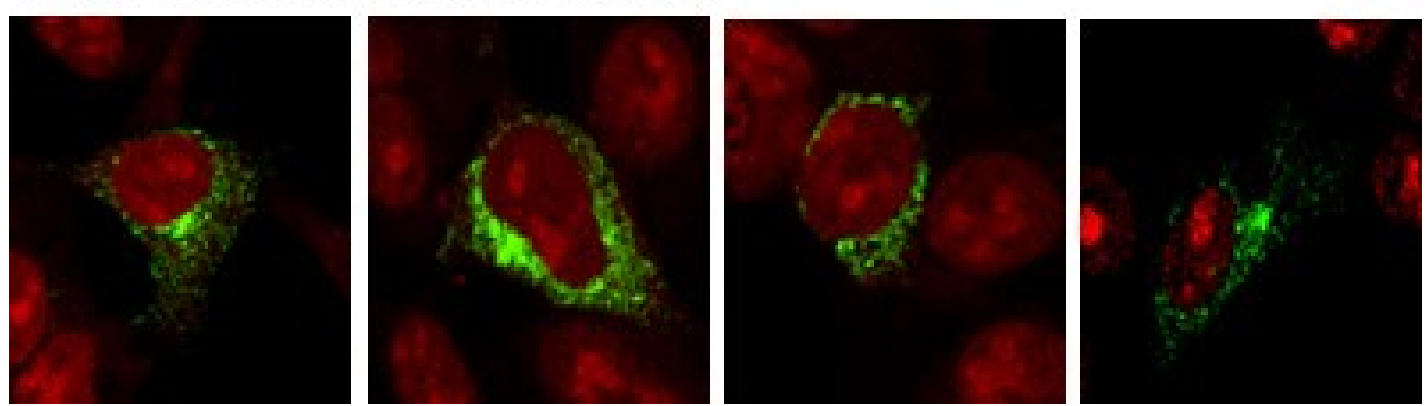

Figure 4-10. Role of NHERF2 in determining iNOS localization inside the cell

iNOS immunostaining in HEK-293 cells with no iNOS expression and without NHERF2 knockdown (upper panel), with iNOS expression and without NHERF2 knockdown (middle panel) and with iNOS expression and NHERF2 knockdown (bottom panel). HEK-293 cells with no NHERF2 knockdown represent cells transduced with scrambled SiRNA. 

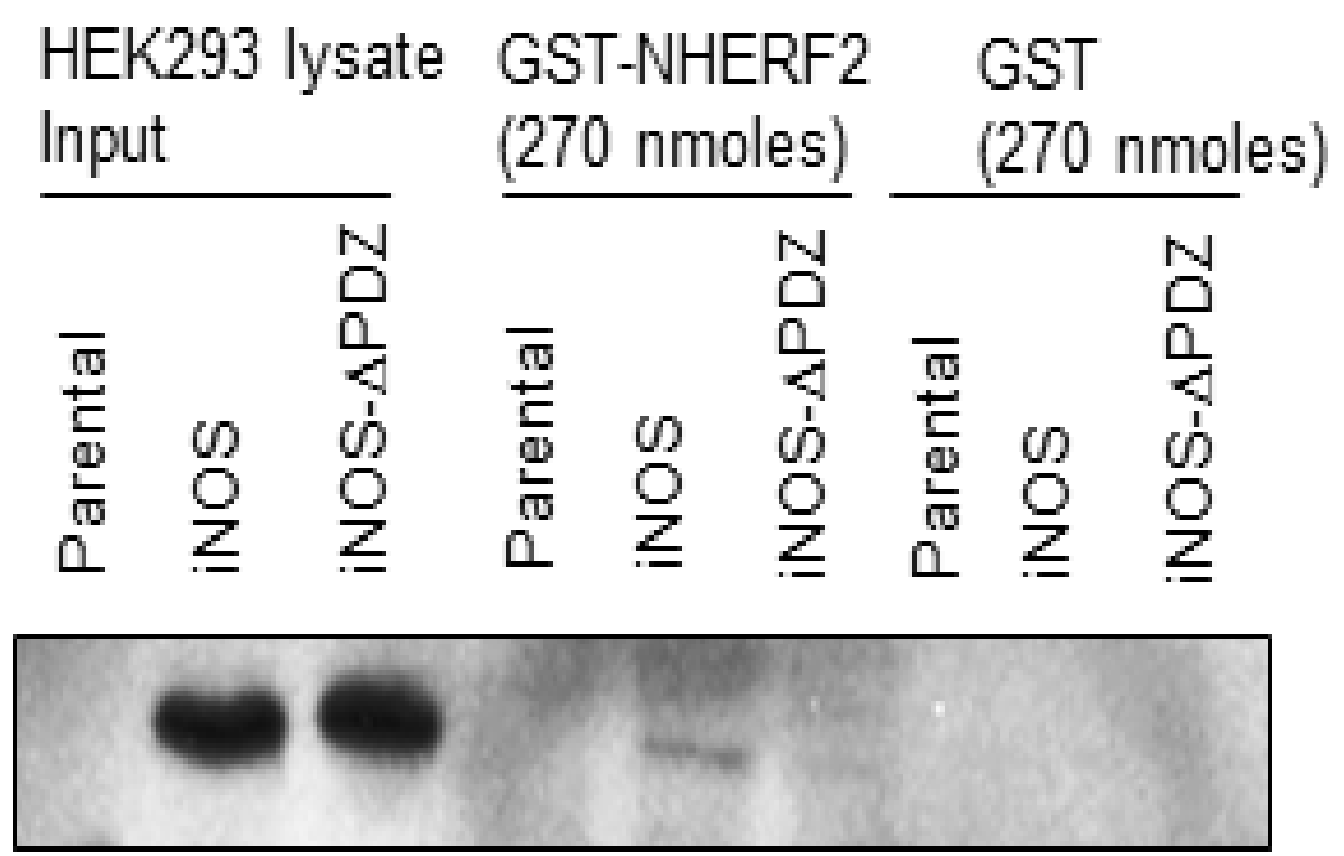

Figure 4-11. PDZ-interaction based iNOS-NHERF2 coupling

Western blot depicting pull-down of full-length iNOS and iNOS- $\triangle \mathrm{PDZ}$ with GSTNHERF2. Removal of PDZ motif disabled interaction of iNOS with NHERF2. 
HT29CL19A (Parental)

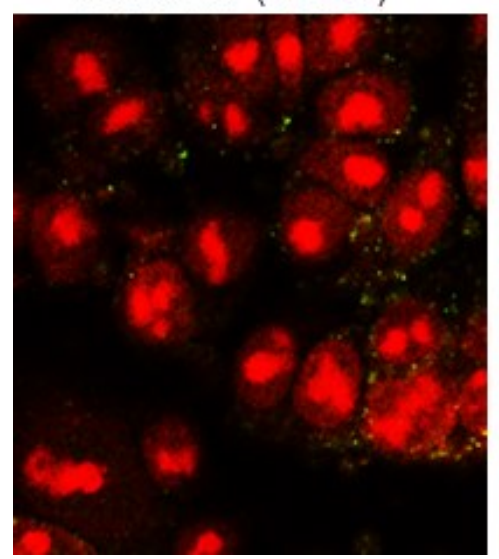

HT29CL19A (iNOS)

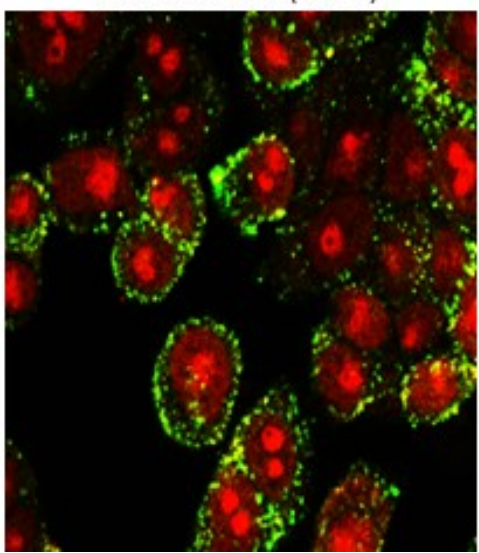

HT29CL19A (iNOS- $A$ PDZ)

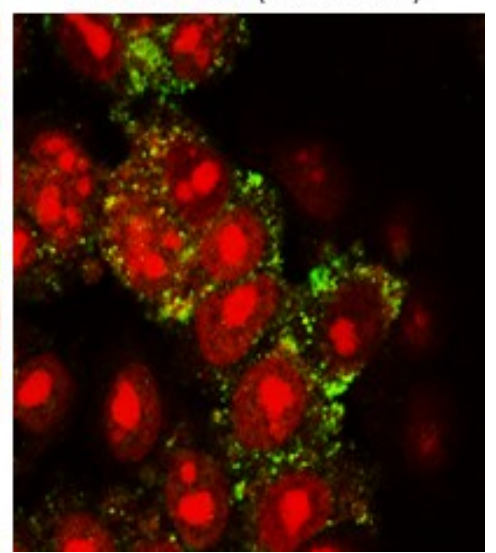

Figure 4-12. PDZ-interaction dependence of iNOS localization in colon epithelial cells

iNOS immunostaining in HT-29CL19A cells with no iNOS expression (left panel), with iNOS expression (middle panel) and with iNOS- $\triangle \mathrm{PDZ}$ expression (right panel). 
rationalized that a macromolecular complex might exist among CFTR, NHERF2, and iNOS with NHERF2 serving as the scaffolding protein. Abilities of NHERF proteins to assemble macromolecular complexes of CFTR of functional relevance have been shown previously and described in detail in Chapter 2. A plausible explanation of NHERFs as preferred scaffolds for CFTR is their predominant sub-apical localizations in polarized epithelial cells (120). Also, NHERFs can anchor enzymatic and regulatory proteins and therefore act as conduits of regulatory influences on CFTR channel activity $(22,30,34$, 121). It is to be noted that NHERF scaffolds are not limited to CFTR and have been shown to assemble many signal-transduction complexes at the plasma membrane which immensely profile vectorial physiology (122). Nonetheless, NHERF mediated complexes of CFTR have been shown to be central to fluid regulation and its ability to function as conductance regulator. To test whether a complex of CFTR-NHERF2-iNOS exists, we performed an in vitro macromolecular formation assay with purified GST-His-S-iNOS Ctail, GST-NHERF2, and Flag-CFTR proteins. Briefly, His-S-iNOS C-tail $(20 \mu \mathrm{g})$ and GST-NHERF2 at two different concentrations (5 and $15 \mu \mathrm{g})$ are mixed first for $1 \mathrm{~h}$ and immobilized on S-beads $(20 \mu \mathrm{l})$. Purified Flag-CFTR is then added to the protein-bead mix and allowed to interact overnight at $4^{\circ} \mathrm{C}$. An assembled protein complex immobilized on S-beads is shown in Figure 4-13 (upper panel). A macromolecular complex of CFTR and iNOS could be inferred from the data shown in Figure 4-13 (lower panel).We found that the complex could only be assembled when NHERF2 was present, and that the formation of this complex increased in a NHERF2-dependent manner Figure 4-13 (lower panel). It correlates with the fact that stoichiometry of CFTR and NHERF scaffold determines the efficiency of CFTR macromolecular assemblies.We proceeded to determine NHERF2 mediated CFTR-iNOS interaction using PLA in HEK293 cells. Briefly, iNOS and Flag-CFTR were overexpressed in HEK-293 cells with and without NHERF2 knockdown. The knockdown process did not impair the ability of the cells to overexpress proteins and equal levels of iNOS and Flag-CFTR proteins were detected in scrambled and NHERF2 knockdown cells. NHERF2 knockdown reduced the amounts of PLA signals in iNOS and Flag-CFTR co-transfected cells by 50\% (Figure 4-14). As mentioned and demonstrated before that tempering with the CFTR and NHERF2 stoichiometry affects CFTR macromolecular assemblies and plausibly control the dynamics of interaction. We used direct sensitized FRET to test if iNOS and CFTR interaction can be altered by controlling the concentration of NHERF2 in live cells. Toward this end, HEK-293 cells were co-transfected with CFP-iNOS and YFP-CFTR and subjected to FRET imaging. N-FRETc signal between CFP-iNOS and YFP-CFTR at the plasma membrane was very weak but could be visualized on a small temperaturebased FRET look up scale (Figure 4-15A). Increasing the expression of NHERF2 by transfecting the cells with different amounts of pCMV-HA-NHERF2 cDNA $(0,0.2,0.5$, 0.75 and $1 \mu \mathrm{g}$ ), enhanced the N-FRETc signals by 2 - to 2.5 -fold compared to the HANHERF2 untransfected cells (Figure 4-15B). Only selective data is presented here to emphasize the concept. Concomitantly, with the presence of greater amounts of NHERF2, there will be more iNOS getting trafficked to the plasma membrane and the possibility of achieving macromolecular associations with CFTR would be higher. Transfection with more than $1 \mu \mathrm{g}$ of HA-NHERF2 could not further increase the FRET signal, implying that the complex was already saturated. 

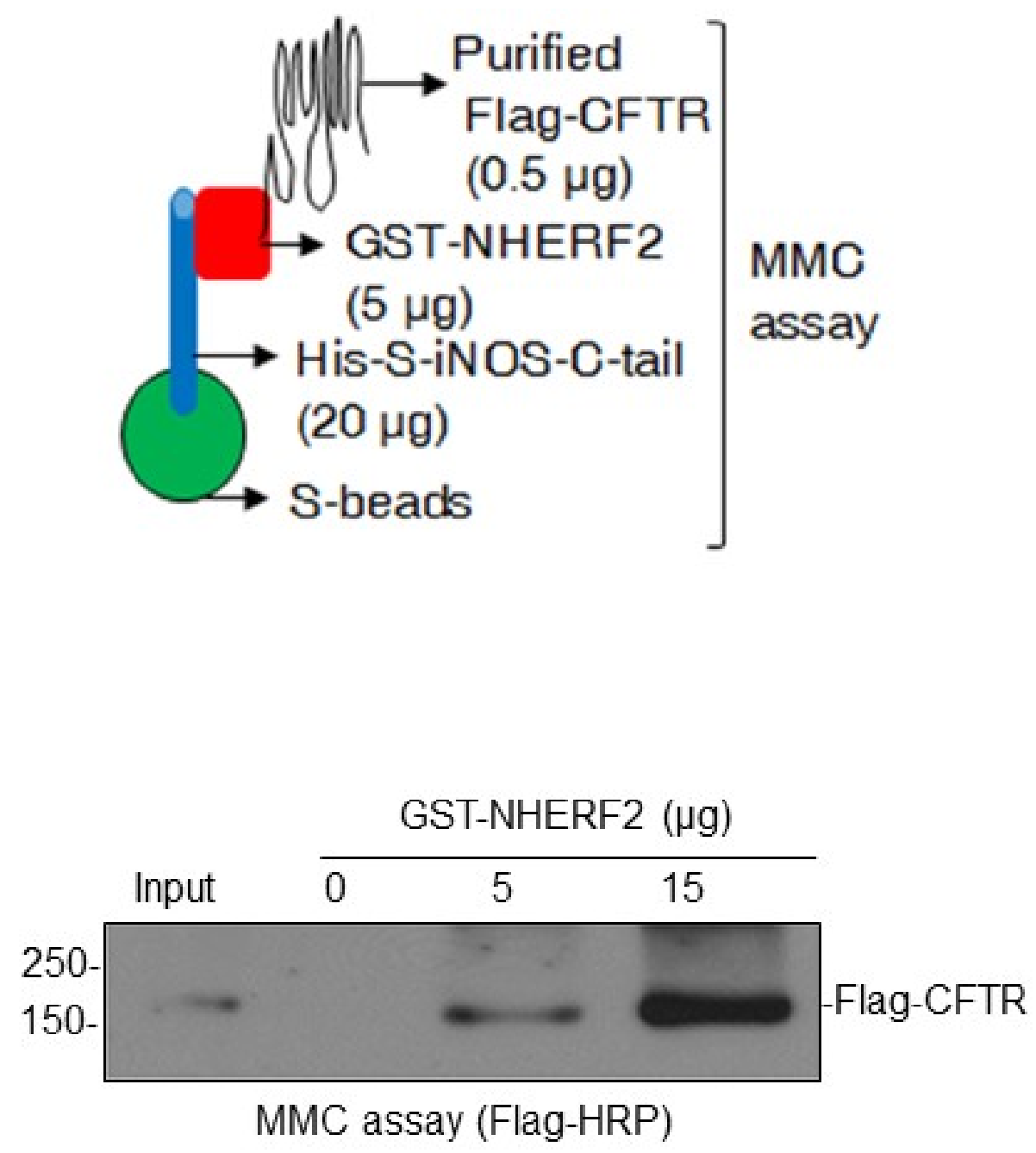

Figure 4-13. CFTR-NHERF2-iNOS macromolecular complex

A pictorial representation of the macromolecular complex assay (upper panel). A macromolecular complex (MMC) was detected in vitro with three purified proteins (HisS-iNOS-C-tail, GST-NHERF2, and Flag-CFTR) (lower panel). 

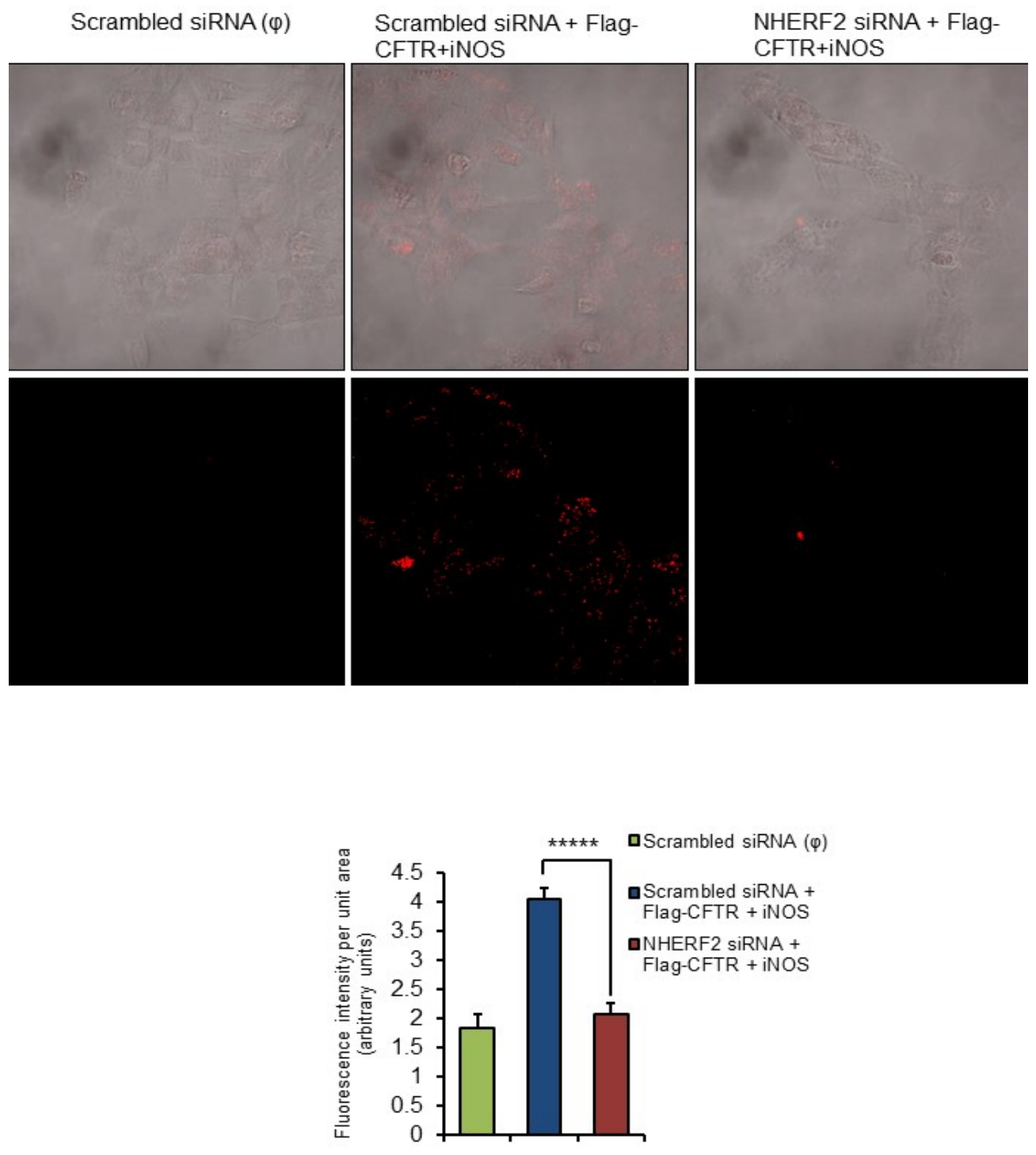

Figure 4-14. Knockdown of NHERF2 expression compromises CFTR-iNOS physical coupling at the plasma membrane

Representative images of the PLA assay in HEK-293 cells with scrambled and NHERF2 knockdown transfected with pcDNA3-empty vector (left, indicated by symbol $\varphi$ ), FlagCFTR and iNOS both (middle and right). PLA signal was quantified as fluorescence intensity and represented in the form of a bar-graph (lower panel). Data represent the mean \pm SEM $(\mathrm{n}=8-49$ regions of interest $) ; * * * * * P<0.00001$ as determined using student's t-test. 


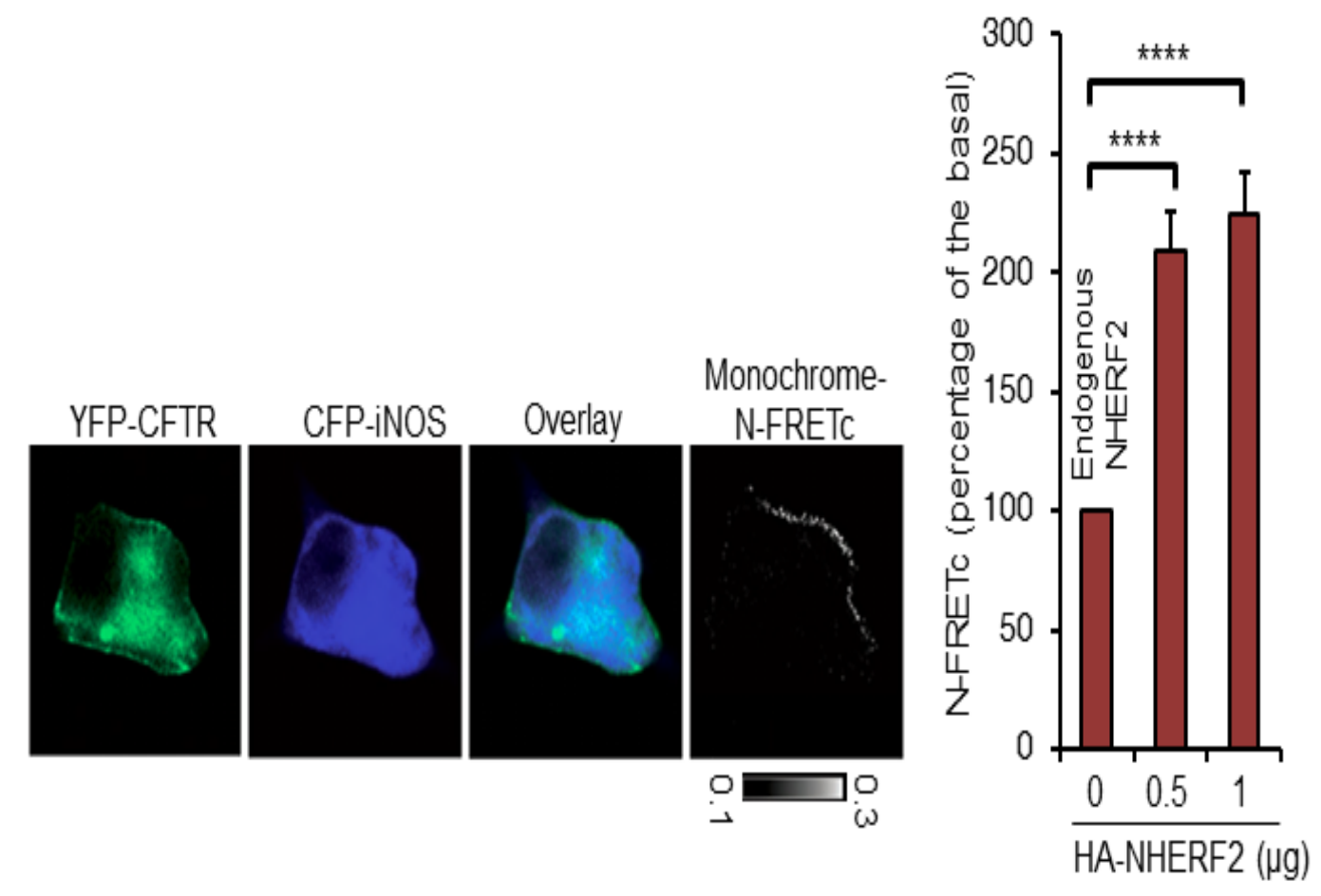

Figure 4-15. Modulating iNOS-CFTR interaction at the plasma membrane

(A) Representative direct sensitized FRET depicting interaction between CFTR and iNOS at the plasma membrane in HEK-293 cells. The cells were single or cotransfected with CFP-iNOS and YFP-CFTR, and the pseudocolor images show the expression. HANHERF2 was also included in the transfection media to increase N-FRETc. N-FRETc between CFP-iNOS and YFP-CFTR at the plasma membrane was shown in a monochrome mode, according to a temperature-based look-up table.

(B) Bar graph represents the increase in N-FRETc between CFTR and iNOS with increasing concentrations of HA-NHERF2. Data represent the mean $\pm \mathrm{SEM}(\mathrm{n}=3$ independent experiments, $8-10$ regions of interest per experiment); $* * * P<0.00001$ as determined using student's t-test. 


\section{iNOS Protein Is Organized into Clusters at or near the Apical Plasma Membrane in Inflammatory Bowel Disorders}

iNOS levels have been shown to be upregulated in colonic biopsies from IBD patients in UC and CD, and enhanced NOS levels and associated disease manifestations are better correlated in UC patients than in Crohn's colitis patients (75). Inflammation patterns in UC vs. CD appear superficially similar but are distinct. Inflammation in CD can be found in any part of the gastrointestinal tract and usually occurs in the forms of patches and extends beyond the mucosa into deeper layers of the gut. In UC, inflammation extends proximally from the rectum and is restricted to the colon, usually occurs in a continuum and affects the mucosal surface only (99). Due to better correlation between iNOS function and pattern of inflammation in UC, the current study focuses on UC. Costello et al. (2005) used human endoscopic biopsies from sigmoid colons of normal individuals and patients suffering from active $\mathrm{CD}$ and $\mathrm{UC}$ to examine the pattern of genome-wide gene expression in IBD (123). The sample size comprised a total of 31 individuals with the data from normal $(n=11), C D(n=10)$ and $U C$ patients $(n=10)$ to be included for the analysis. The transcripts were datamined corresponding to iNOS, NHERF2, and CFTR in active UC-affected biopsies and were compared with those in the normal control samples. The rationale behind including CFTR in the analysis is based on three facts. First, IBD patients have severe diarrhea and CFTR function is directly correlated with the development of diarrhea. Whether CFTR contributes to diarrhea in IBD is not known. Second, we have demonstrated that iNOS binds NHERF2 and previously, several studies have described interactions between CFTR and NHERF proteins. Therefore, it becomes reasonable to explore the possibility of iNOS and CFTR being connected to NHERF2. Third, iNOS can be functionally coupled to CFTR chloride channel function as iNOS can stimulate cGMP production via the canonical NO-cGMP pathway and it is already known that cGMP can potentiate CFTR activity. We determined that iNOS expression level was 2-fold higher in UC patients compared to the normal controls (Figure 4-16). The change in CFTR expression levels was not significantly altered, and NHERF2 expression slightly increased in UC patients compared to the normal individuals (Figure 4-16). This correlates with the prevailing data on iNOS activity in IBD-affected patients which is increased by 2-3 fold and also, with the determined expression changes in CFTR in IBD which have been not quite consistent. Change in expression of NHERF2 in IBD-affected tissue has not been determined previously and our current analysis demonstrates that NHERF2 levels are not significantly altered in IBD.

Histological staining of normal and UC-affected human colon tissues showed disrupted mucosal architecture and massive infiltration by the immune cells (Figure 4-17). Using immunohistochemical analysis, we confirmed the increase in iNOS levels and its predominant localization at or near the apical plasma membrane in UC-affected colon compared to normal colon (marked arrows in upper panel; Figure 4-18). NHERF2 was found to localize predominantly at or near the apical plasma membrane in both normal and UC colons (marked arrows in middle panel; Figure 4-18) and the expression of CFTR protein was unchanged in UC-affected colon compared to the normal ( marked arrows in lower panel; Figure 4-18). In order to quantitate the relative changes in protein 


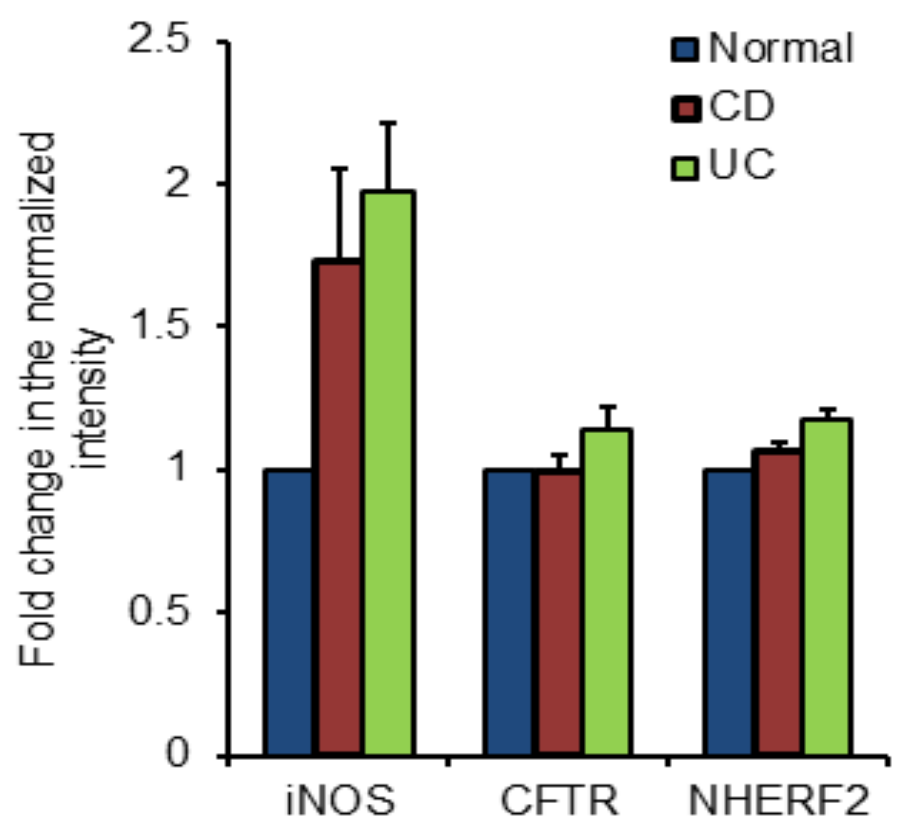

Figure 4-16. iNOS, CFTR and NHERF2 expression profiles in UC-affected human colon tissue

Normalized intensities of mRNA expression of iNOS, CFTR, and NHERF2 from human mRNA expression data sets in colonic biopsies from normal and active IBD-suffering individuals. Data represent the mean $\pm \operatorname{SEM}$ ( $\mathrm{n}=10-11$ per group). 
Normal human colon

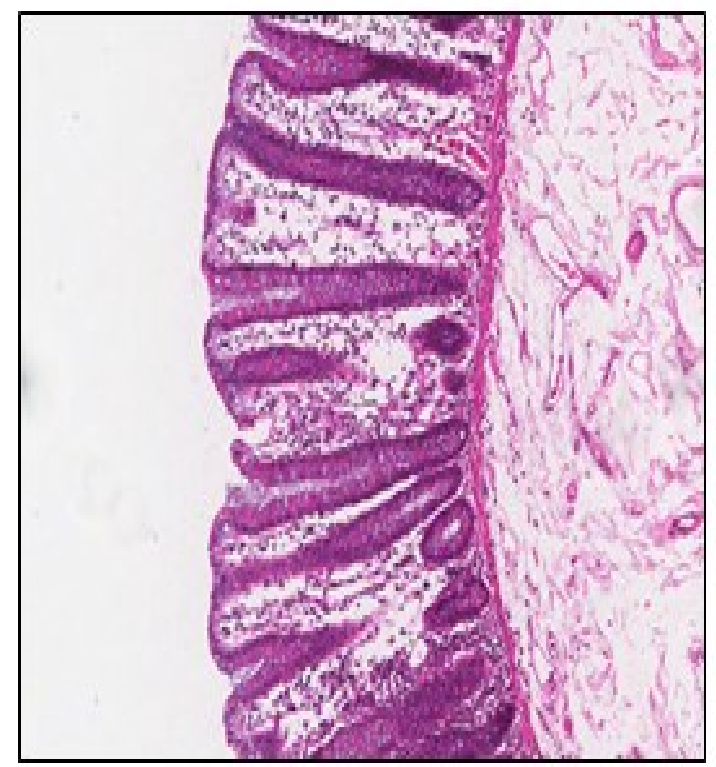

UC-affected colon

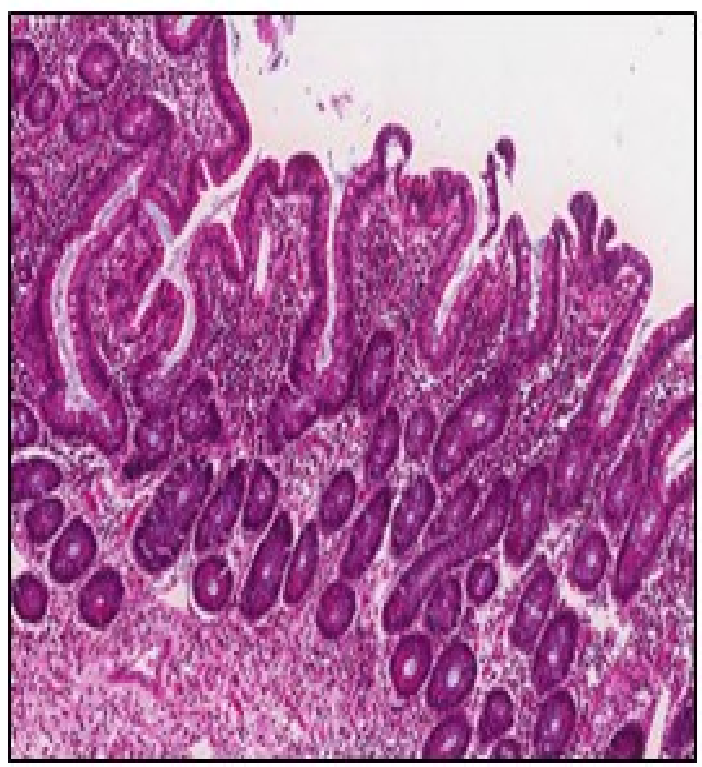

Figure 4-17. H\&E stained normal and UC-affected human colon tissue sections 
A

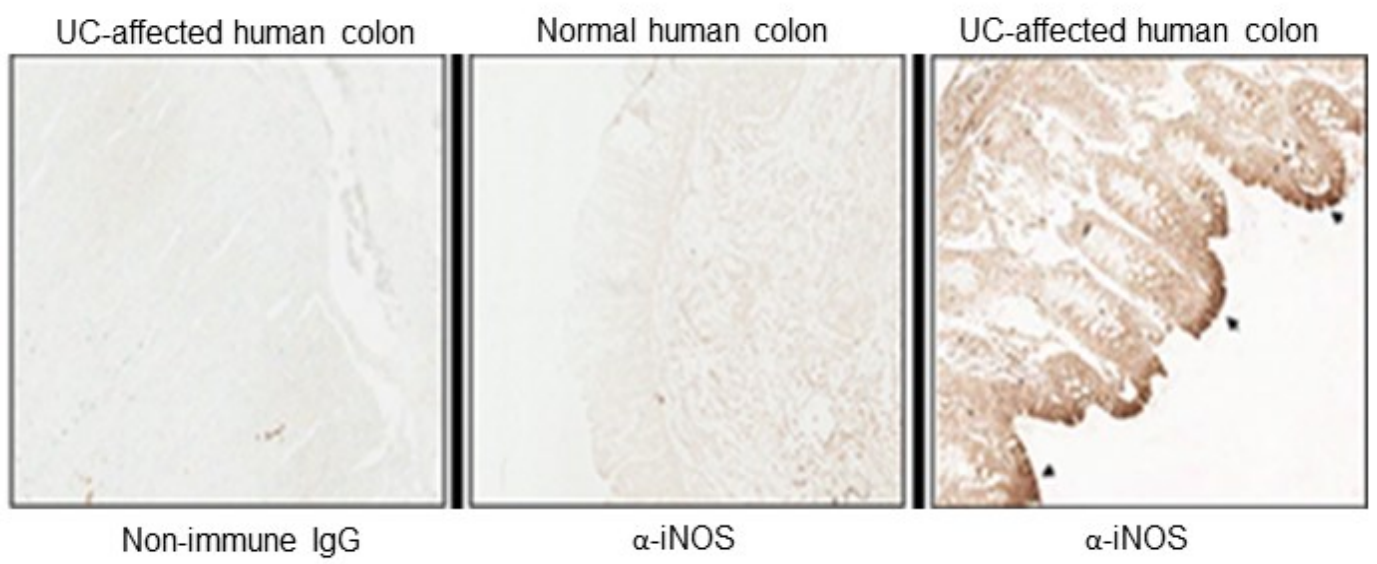

B

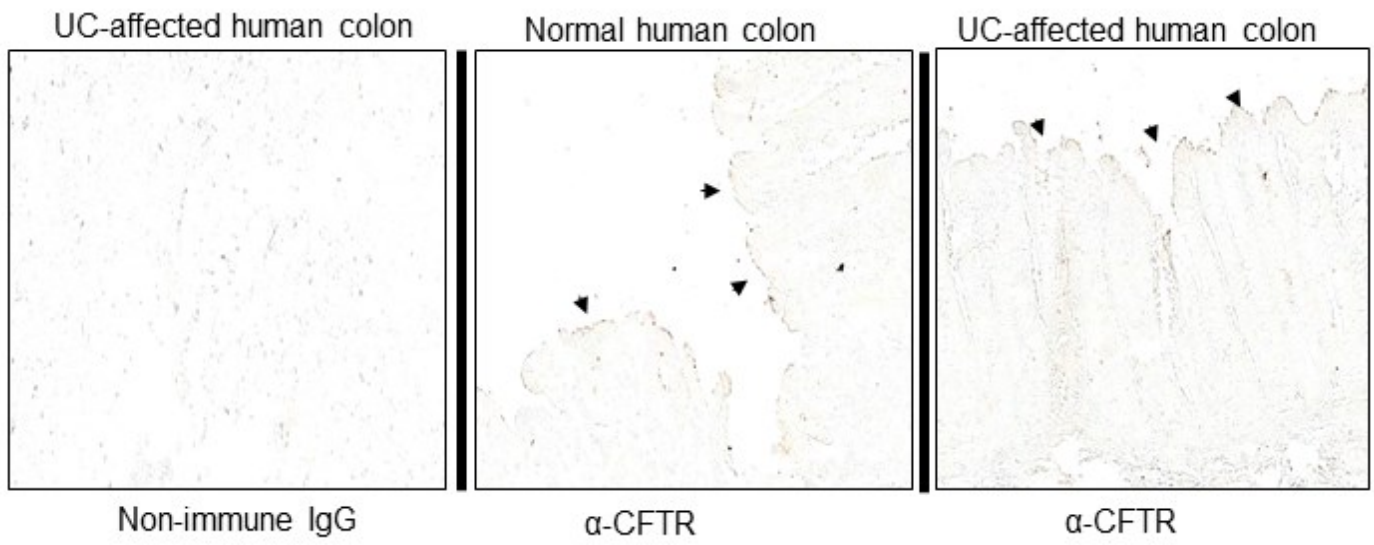

C

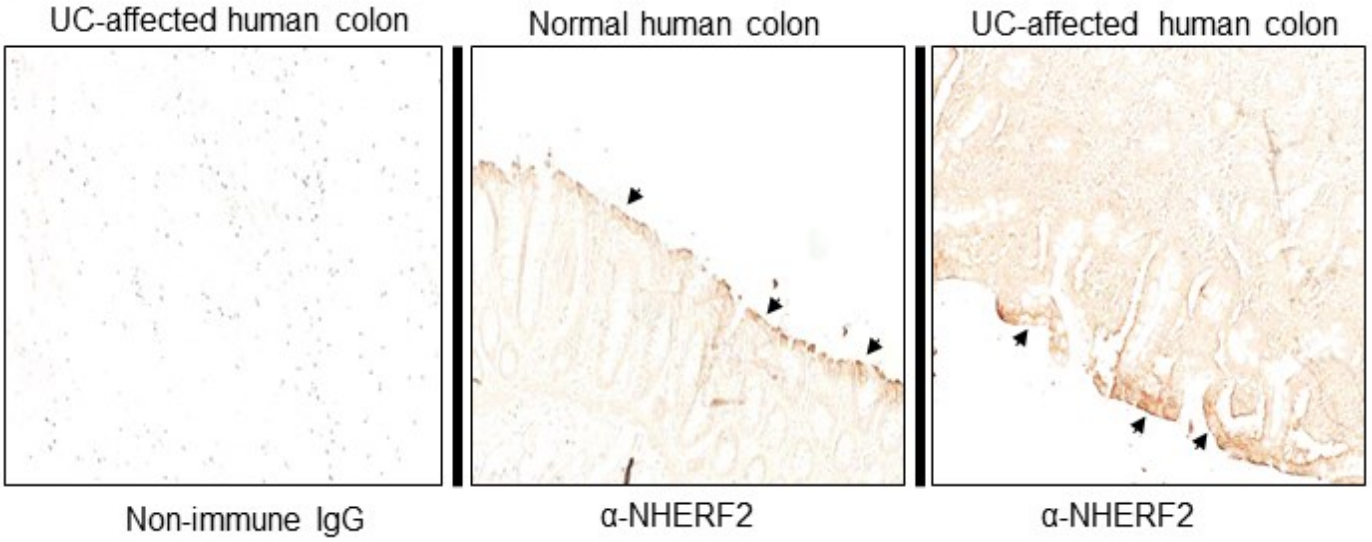

Figure 4-18. Immunostaining for iNOS, CFTR and NHERF2 expression in normal and UC-affected human colon sections 
expression of iNOS, CFTR and NHERF2 ImageJ was used and mean gray values were obtained as depicted in the form of bar-graphs in Figures 4-19A through 4-19C.

In order to demonstrate that there are high amounts of iNOS associated with the plasma membrane in IBD, Total internal reflection microscopy (TIRFM) was performed on the normal and UC-affected tissue present close to the coverslip. TIRFM can visualize single molecule fluorescence with high resolution to capture events taking place at or near the plasma membrane within a span of $200 \mathrm{~nm}$ (124). The thickness of plasma membrane is approximately $8 \AA$. TIRFM is based on the generation of an evanescent wave at the water-glass interface as a result of the phenomenon of total internal reflection. The evanescent electromagnetic field decays exponentially from the interface and can penetrate up to $200 \mathrm{~nm}$ into the sample medium and therefore, falls within the range of visualizing basal plasma membrane. UC-affected and -unaffected tissues were stained with primary mouse monoclonal iNOS antibody followed by anti-mouse Alexa-fluor-488 secondary antibody. Antibody stained slides were excited at $488 \mathrm{~nm}$ and imaged using the TIRF module of the microscope. Using TIRFM, we could identify iNOS clusters at the plasma membrane in UC biopsies suggestive of its involvement in spatially restricted signaling complexes at the plasma membrane (marked arrows; Figure 4-20). The data also points to the direction that in IBD epithelial cell membranes are enriched with iNOS; therefore, at these membranes the iNOS-NO-sGC-cGMP pathway will be predominantly operative.

In order to validate our in vitro data on the pathophysiological existence of iNOSNHERF2 interaction, an IBD simulating animal model of chemically-induced colitis involving oral administration of DSS was developed. Specifically the model was tested in C57BL/6 Nherf $2^{+/+}$vs. Nherf $2^{-/-}$mice and the immunoblot shows the absence of NHERF2 in Nherf $2^{-/}$mice (Figure 4-21A). Kidney tissue was included for the western blot analysis as NHERF proteins are highly abundant in the kidney. Please note that the DSS-colitis model closely relates to UC conditions and the associated iNOS-upregulation and is also the most appropriate model to study UC (75). DSS administered in the water for 7 days induces severe acute colitis in mice. Colon tissues from different groups were excised and processed for histological staining. The microscopic sections of the DSSchallenged colons from the Nherf $2^{+/+}$and Nherf $^{-/-}$mice groups showed a range of histopathological features characteristic of DSS-induced colitis, including mucosal edema, epithelial cell and crypt damage, and immune cell infiltrates characterizing an inflammatory colon (Figure 4-21B). Histological scores were also quantified and no significant differences were observed between Nherf $^{+/+}$and $N$ herf $2^{-/-}$mice (Figure 4-21B). iNOS-specific immunostaining confirmed similar upregulation of iNOS in response to DSS treatment in both $N$ herf $2^{+/+}$and $N$ herf $2^{-/}$mice compared to untreated mice (Figure 4-22). Also, DSS-challenged mouse colons had upregulated iNOS levels at the apical plasma membrane of the gut epithelia (marked arrows; Figure 4-22). Therefore, the absence of NHERF2 does not affect the expression of iNOS under colitis. Therefore, consequences of depleted NHERF2 will be on iNOS trafficking to the plasma membrane as demonstrated in Figures 4-10 and 4-12. Moreover, it will also lead to reduced iNOS involvement in highly localized signaling at specialized plasma membrane microdomains. 
A

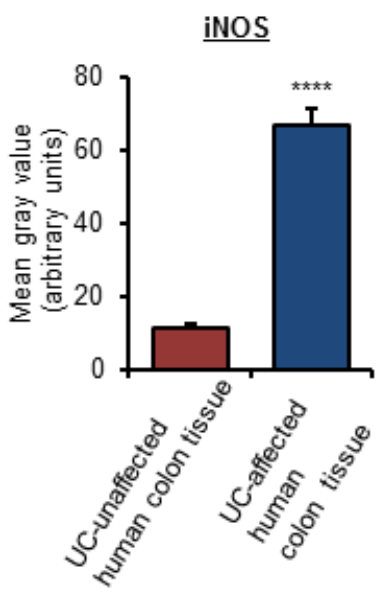

B

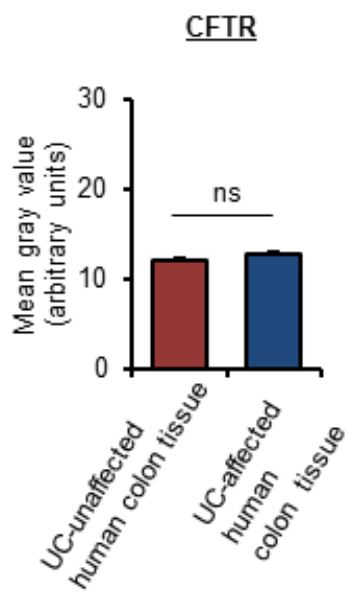

C

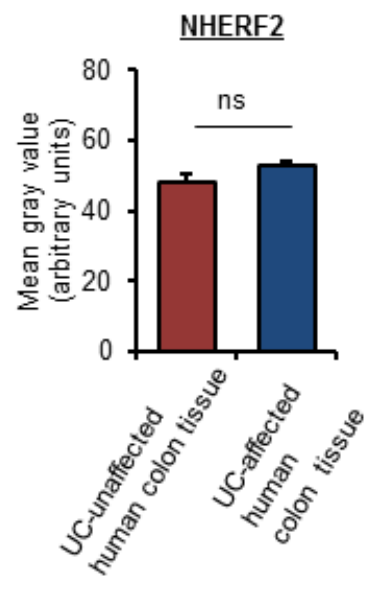

Figure 4-19. Quantitated iNOS, CFTR and NHERF2 expression in normal and UC-affected human colon sections

Bar-graphs represent quantification of (A) iNOS, (B) CFTR and (C) NHERF2

expressions as mean gray values in normal and UC-affected human colon sections. Data represent the mean $\pm \mathrm{SEM} ; * * * * P<0.001, \mathrm{~ns}=$ non-significant determined using student's t-test. 


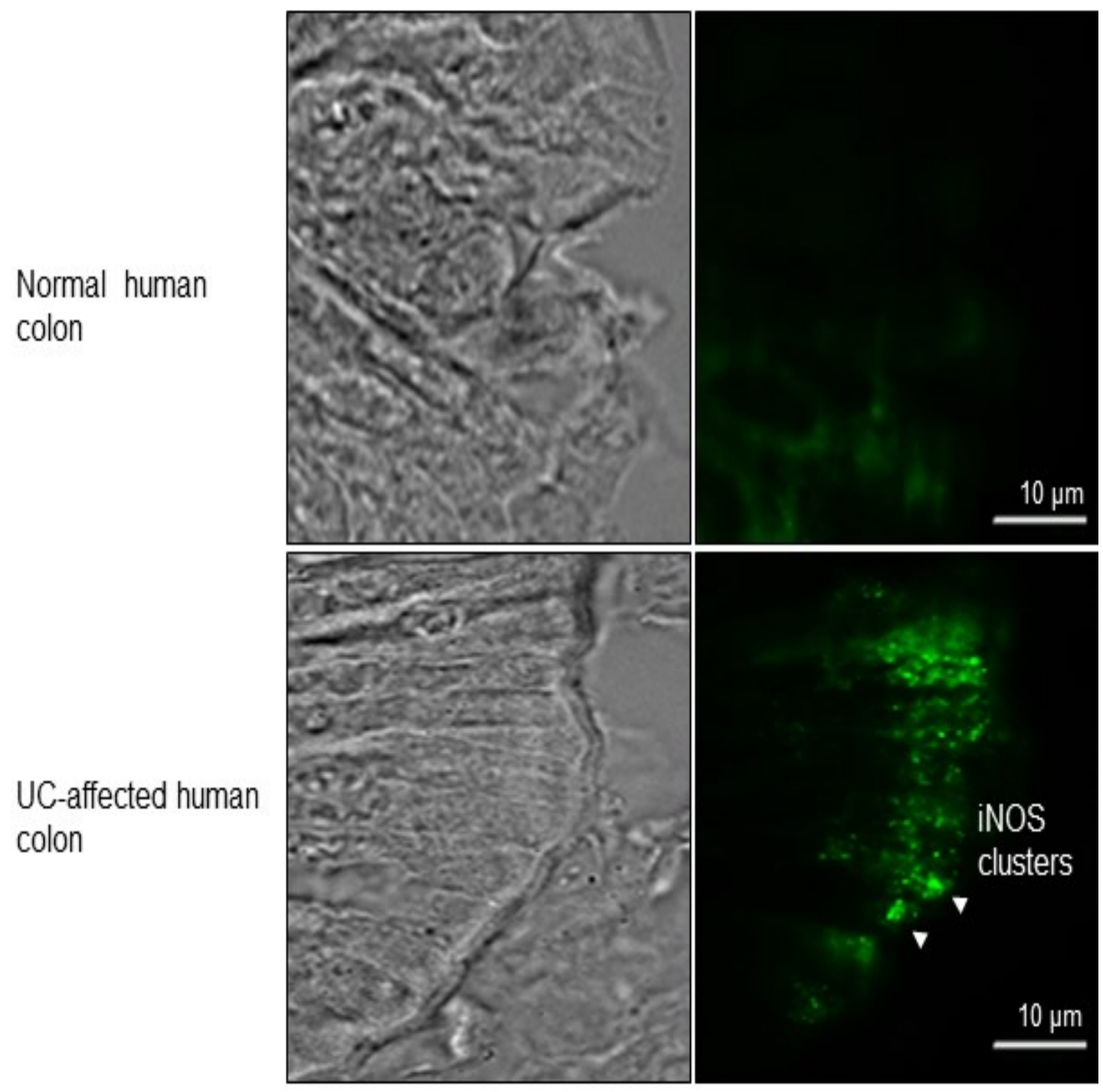

Figure 4-20. iNOS clusters at the plasma membrane in UC-affected human colon epithelia

TIRF images of normal and UC-affected human colon tissues immunostained for iNOS depicting organization of iNOS into clusters in UC-affected human colon tissue (marked arrows). 
A

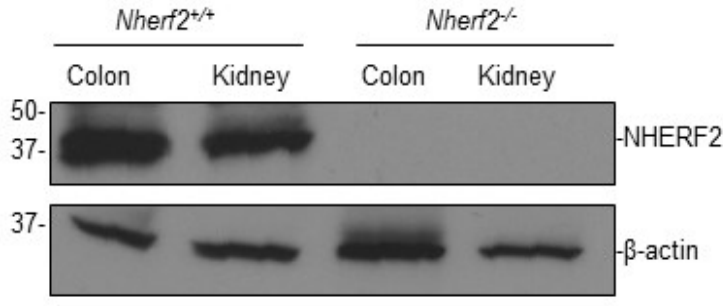

B
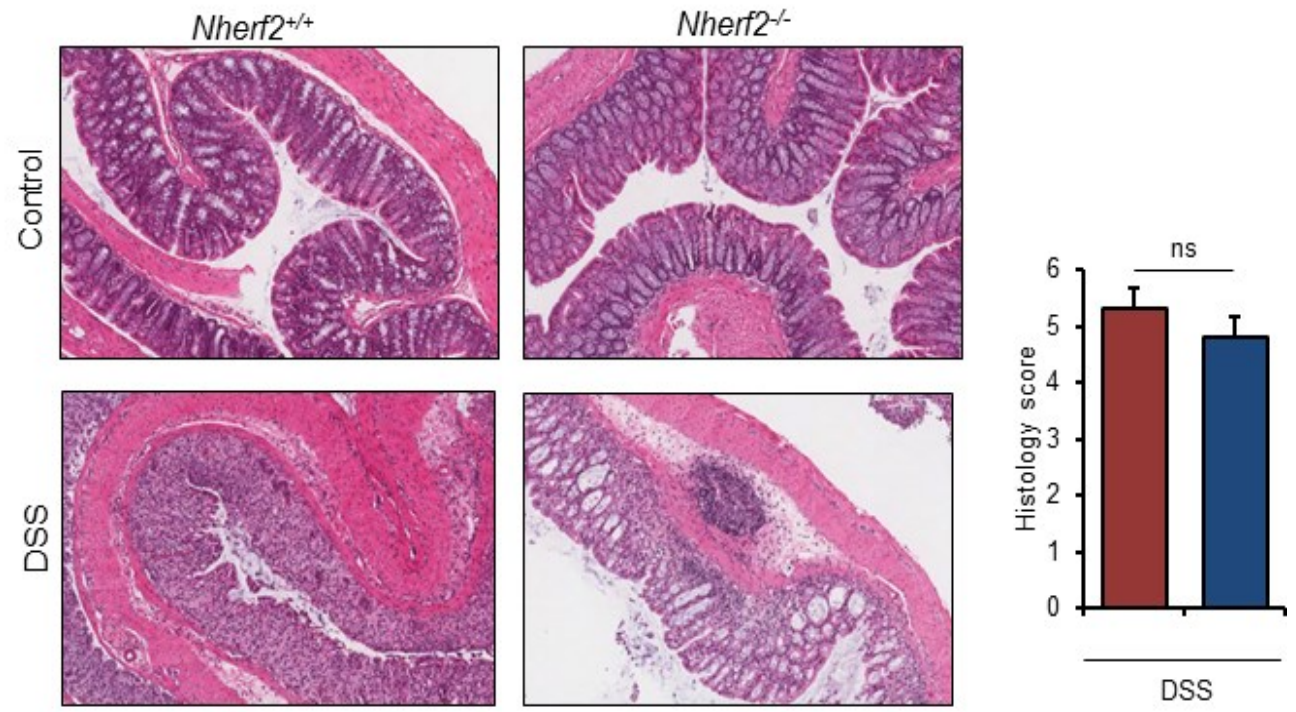

H\&E staining

Figure 4-21. DSS-colitis in $N h e r f 2^{+/+}$and $N h e r f 2^{-/-}$mice

(A) Immunoblot for NHERF2 expression in Nherf $2^{+/+}$and $N h e r f 2^{-/}$mouse kidney and colon lysates. (B) H\&E stained untreated (upper panel) and DSS-treated (lower panel) Nherf $2^{+/+}$(left panel) and Nherf $2^{-/-}$(right panel) mouse colon sections. Bar graph represents the histology score of the colon sections from DSS-treated Nherf $2^{+/+}$and Nherf $^{-/-}$mice. Data represent the mean \pm SEM ( $\left.\mathrm{n}=3-5\right)$; ns= non-significant. 

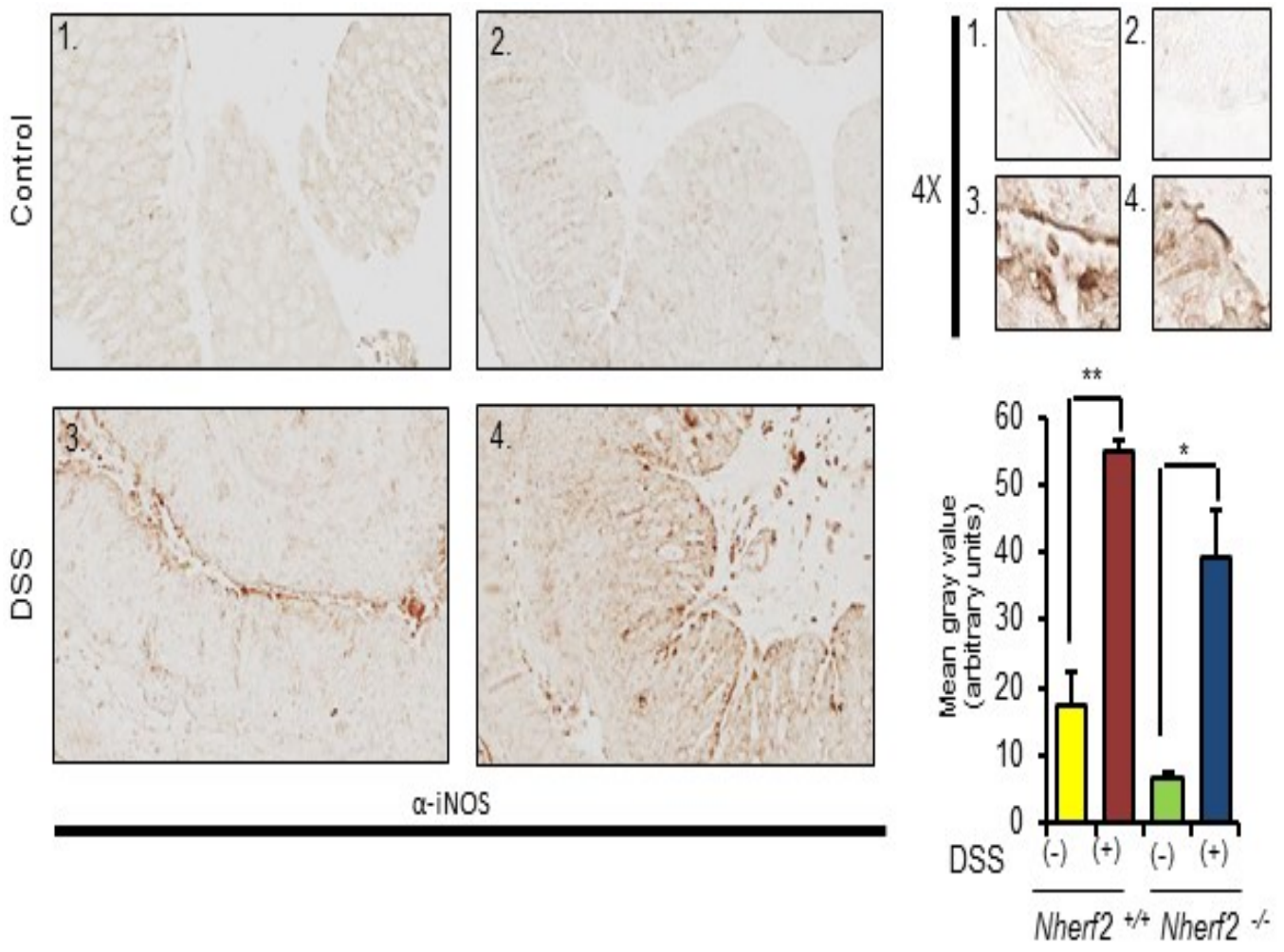

Figure 4-22. iNOS expression in DSS-colitis

iNOS specific immunostained DSS-treated and untreated $N$ herf $2^{+/+}$and $\mathrm{Nherf}^{\mathrm{C}^{-/}}$mouse colon sections. Numbers 1-4 indicate the representative images of the four groups of mouse. Bar graph represents the quantitation of the iNOS expression in the sections from DSS-treated and untreated Nherf $2^{+/+}$and $N$ herf $2^{-/-}$mice. Data represent the mean \pm SEM ( $\mathrm{n}=4$ regions of interest); ${ }^{*} P<0.05, * * P<0.01$ determined using student's t-test. 
Interaction of iNOS and NHERF2 in UC-affected human colon tissue was qualitatively determined using proximity ligation assay (PLA). The interaction between two proteins appears as red PLA signal if the specific antibodies binding to the proteins are proximal to each other within a span of $40 \AA$ and many previous studies have validated the application of the assay in determining protein complexes and colocalizations in vivo $(125,126)$. The advantage of this assay is the ability to determine protein-protein interactions in tissues rather than limiting to a cell system and also, the assay can be used with the fixed tissues and cells unlike FRET-based assays.

Fundamental approach of the PLA assay is labeling the cells or tissue with the antibodies specific to proteins of interest, followed by addition of species specific probes conjugated with oligonucleotides. If the proteins are present and interacting in the right proximity required for successful PLA, there will be a polymerization reaction of the conjugated nucleotides, incorporating fluorescent nucleotides and generating a signal termed as the PLA signal. We observed significant amount of PLA signals at the plasma membrane of the colon epithelial cells in UC-affected tissues signifying the interaction between iNOS and NHERF2 (lower panel, marked arrows; Figure 4-23) while almost no PLA signal was observed in normal human colon tissues (upper panel; Figure 4-23).

In order to demonstrate the physiological existence of a CFTR-NHERF2-iNOS macromolecular complex in the DSS-induced colitis model, colon mucosal epithelial cells scraped from the control and DSS-treated animals were used and CFTR and iNOS were probed for in the immunoprecipitated complex with purified GST-NHERF2 immobilized on glutathione beads. CFTR could be detected in a complex with GSTNHERF2 in both control and DSS-treated colon mucosal epithelial cells while iNOS was detected in the complex in DSS-treated tissue only (Figure 4-24). To ensure the physiological existence of a CFTR-NHERF2-iNOS macromolecular association, coimmunoprecipitation was performed in DSS-treated Nherf $2^{+/+}$mouse colon with iNOS specific antibody and CFTR and NHERF2 were detected in the complex with iNOS (Figure 4-25). Thus, with the evidence of induced expression of iNOS at the apical plasma membrane possibly due to its interaction with NHERF2 in IBD, there is a high probability of involvement of iNOS in contributing to altered vectorial physiology in the IBD gut.

\section{iNOS Potentially Constitutes cGMP Microdomains}

Because we previously observed that iNOS is overexpressed at the apical plasma membrane in gut epithelial cells, we tested whether iNOS contributes to cGMP production at the plasma membrane. To this end, ratiometric FRET was performed to monitor cGMP dynamics in HEK-293 cells expressing a membrane-bound cGMP sensor, $\mathrm{m}$-Cygnet 2.1. Ratiometric FRET is a FRET-based procedure customized to monitor the changes in the levels of small molecules such as $\mathrm{Ca}^{2+}$, cAMP and cGMP based on the specific sensitivities towards these molecules of unimolecular FRET-based sensors which comprise of a donor and acceptor fluorophore in the same molecule (127-129). cGMPspecific sensors termed as Cygnets are very sensitive to cGMP levels in the nanomolar range and allow detection of spatial associations of cGMP production inside the cell 
Normal human colon
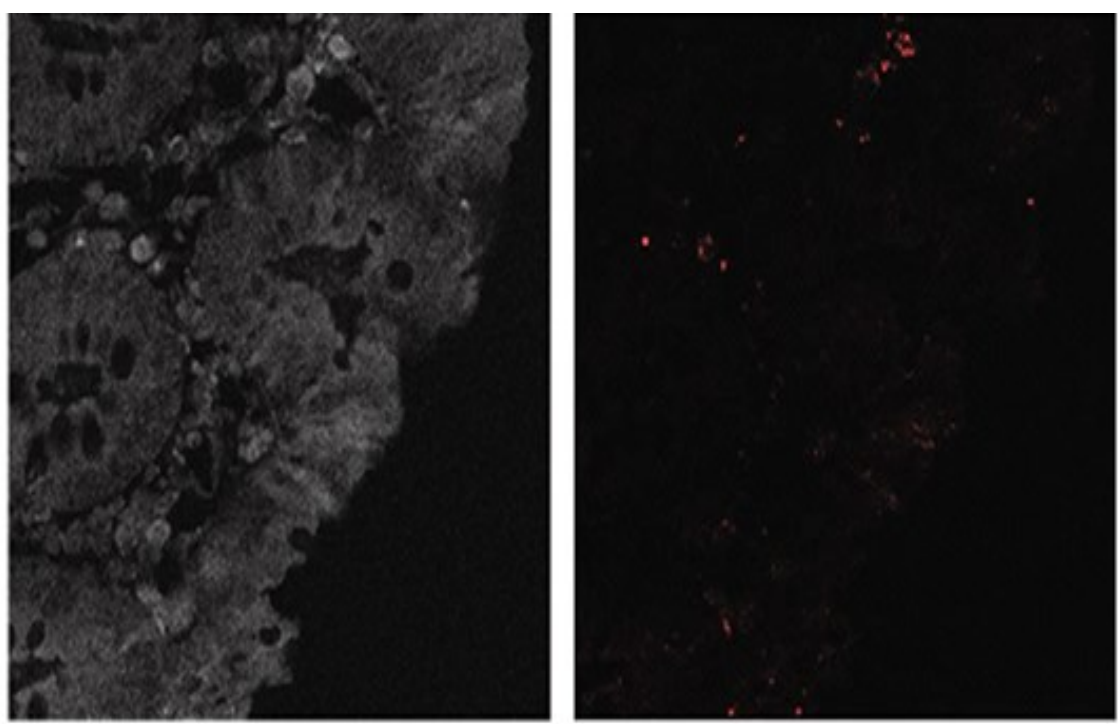

UC-affected human
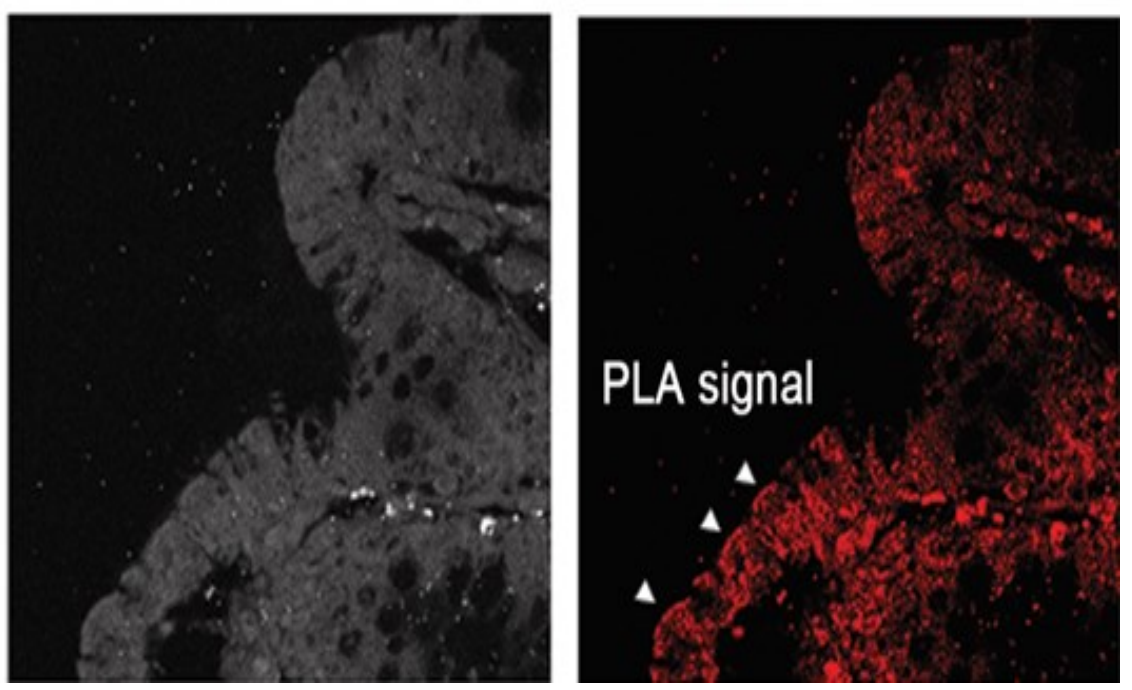

Figure 4-23. iNOS-NHERF2 interaction in UC-affected human colon tissue

PLA in human colon tissue sections depicting interaction between iNOS and NHERF2 in UC-affected human colon tissue sections appearing as a red signal termed the PLA signal (marked arrows). 


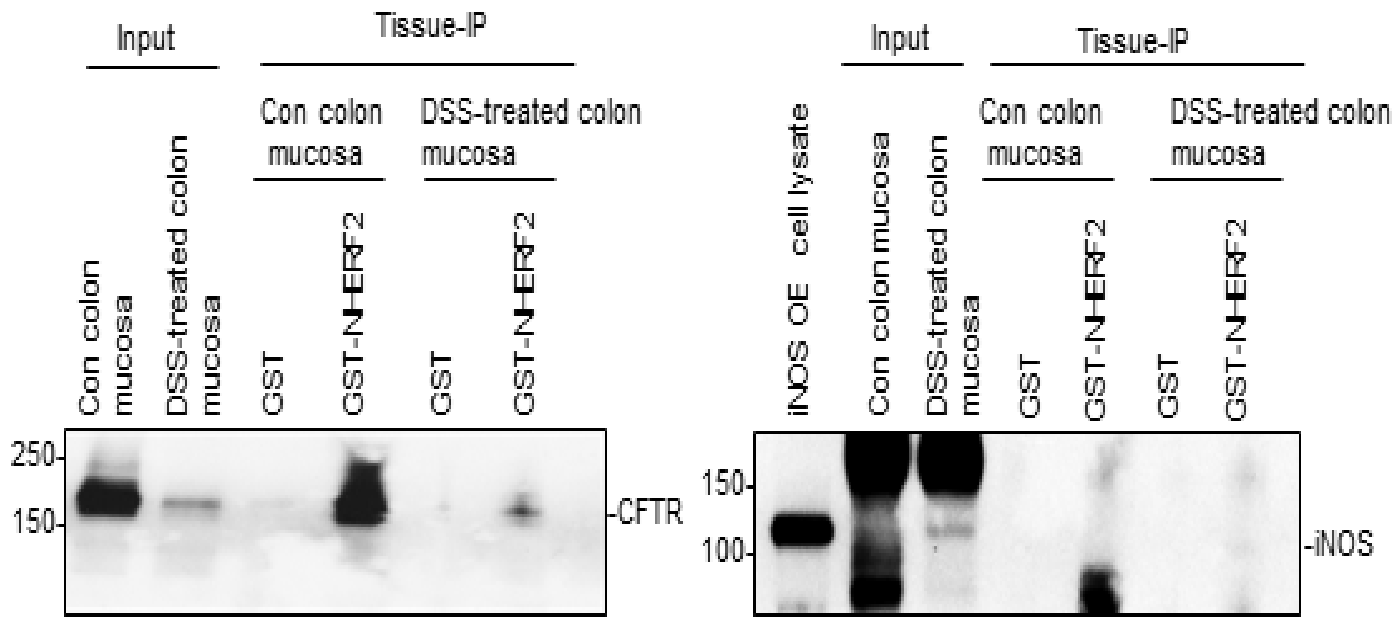

Figure 4-24. Pull-down of CFTR and iNOS using purified GST-NHERF2 in DSStreated colon mucosa

Western blot data depicting pull-down of proteins from colon mucosa tissue lysate from DSS-treated and untreated mice with purified GST-NHERF2 $(20 \mu \mathrm{g})$ and probed for CFTR (left) and iNOS (right). 


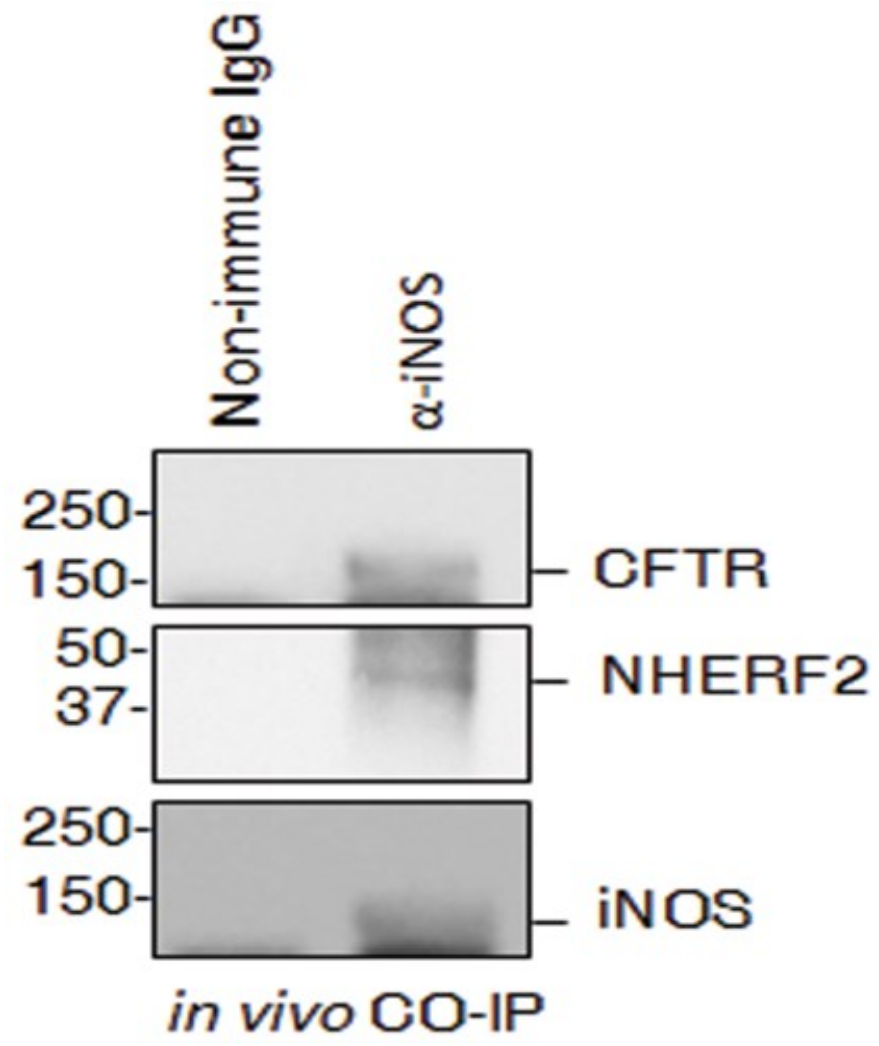

Figure 4-25. Immunoprecipitation of CFTR-NHERF2-iNOS macromolecular complex in DSS-treated colon tissue

Western blot data depicting colon tissue lysate from DSS-treated mice coimmunoprecipitated with anti-iNOS antibody and probed for CFTR and NHERF2. 
(130). Increase in the cGMP is monitored by calculating the ratio of CFP/FRET which has been designed to increase upon binding of cGMP to the sensor. The membrane-bound sensor was generated by inserting the myristoylation-palmitoylation site into the Nterminus of cygnet 2.1 backbone sequence which anchors the protein in the plasma membrane (Figure 4-26). Using this sensor we can specifically monitor cGMP dynamics at the plasma membrane. There was an increase in cGMP levels at the plasma membrane (equivalent to increase in CFP/FRET ratio) upon adding the cGMP-elevating agent, zaprinast, and cells that overexpressed iNOS showed a higher baseline and zaprinastinduced CFP/FRET ratio (i.e., level of cGMP is high) at the plasma membrane (Figure 4-27), all of which suggested that iNOS contributes to cGMP generation at the plasma membrane. Although, it is understood that NO is quite a diffusible molecule however, the predominant association of iNOS at the plasma membrane as clusters in UC colon epithelia corroborates the idea of enrichment of the membrane with localized NO signaling. The evidence of increased cGMP in the presence of iNOS at the plasma membrane suggests close proximity of iNOS and soluble guanylate cyclase and the feasibility of localized generation of cGMP at the membrane. Therefore, it can be concluded that compartmental NO-cGMPsignaling may exist at the plasma membrane.

It is understood that iNOS-governed microdomains would involve an active NOcGMP pathway with the sGC present in the proximity. Since cGMP is a known activator of CFTR chloride channel function, we tested the impact of iNOS overexpression on CFTR function. Towards this end, cGMP modulators, zaprinast and sildenafil, which are phosphodiesterase-5 inhibitors (PDE5-inh; PDE5 is a cGMP-specific diesterase) were used in various cell culture studies. An added explanation to the use of PDE5-inh is the close communication and colocalization of NOS-sGC machinery with PDE5 as demonstrated previously in cardiac myocytes $(131,132)$. We detected significant amounts of PDE5 in HEK-293 and HT29-CL19A cells and epithelial cells from pig colon (Figure 4-28). It was mostly detected as a cytosolic protein with small amounts of the protein associated with the membrane fraction (Figure 4-28; left).

\section{iNOS Physical Interaction with CFTR Forms the Basis of CFTR Channel Activity Potentiation}

CFTR is localized primarily at the apical plasma membrane of gut epithelial cells and can be found enriched in the isolated membranes of pig colon epithelial cells as shown in Figure 4-29 (34). In view of apical localization of iNOS and its involvement in cGMP production at the apical plasma membrane, it is likely that iNOS functionally couples with CFTR and augments CFTR function at the plasma membrane via the NOcGMP pathway. CFTR-mediated halide influx was determined in HEK-293 cells with (here referred to as HEK-293-N) or without (here referred to as HEK-293-S) NHERF2 knockdown and overexpressing Flag-CFTR, iNOS and a YFP-based halide sensor, pCDNA3-TM-Cl'. CFTR mediated $\mathrm{I}^{-}$influx was monitored in response to extracellular addition of zaprinast $(250 \mu \mathrm{M})$ in $136 \mathrm{mM} \mathrm{NaI}$ which activated CFTR function and lead to decreased intracellular YFP fluorescence (133). CFTR-dependent halide flux in response to zaprinast reduced by $28 \%$ in the cells having the partially compromised 
cygnet 2.1

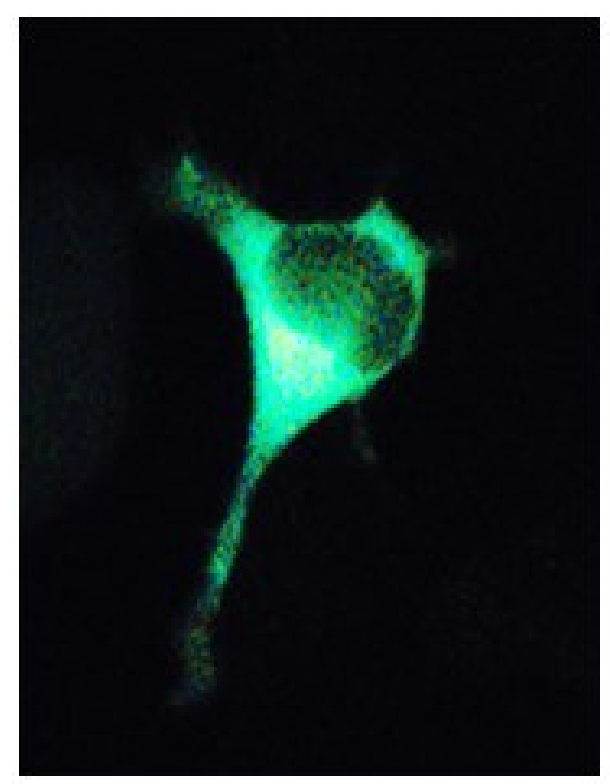

m-cygnet 2.1
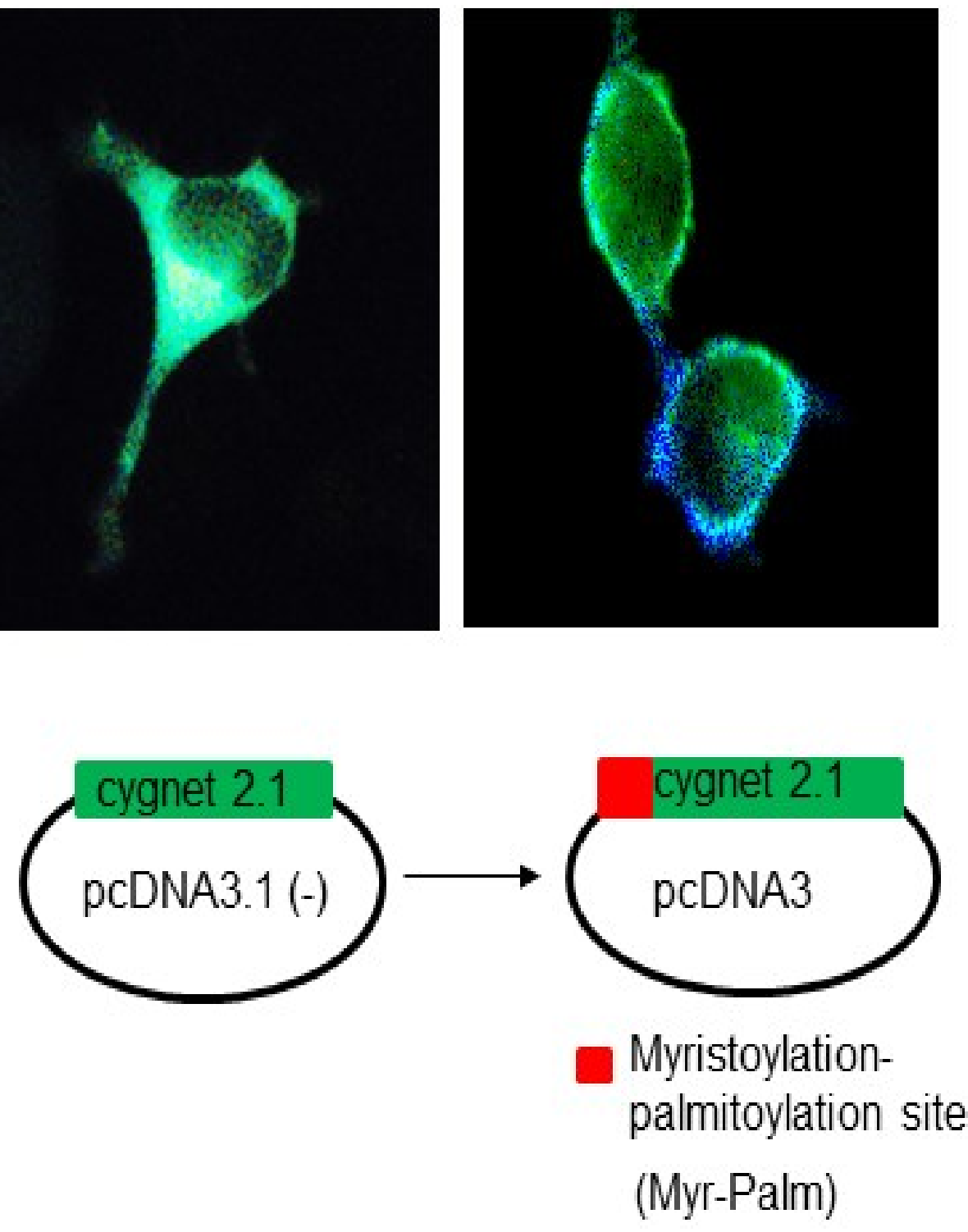

Figure 4-26. Generation of m-cygnet 2.1

Schematics of how membrane-bound cGMP sensor m-cygnet 2.1 was generated. 

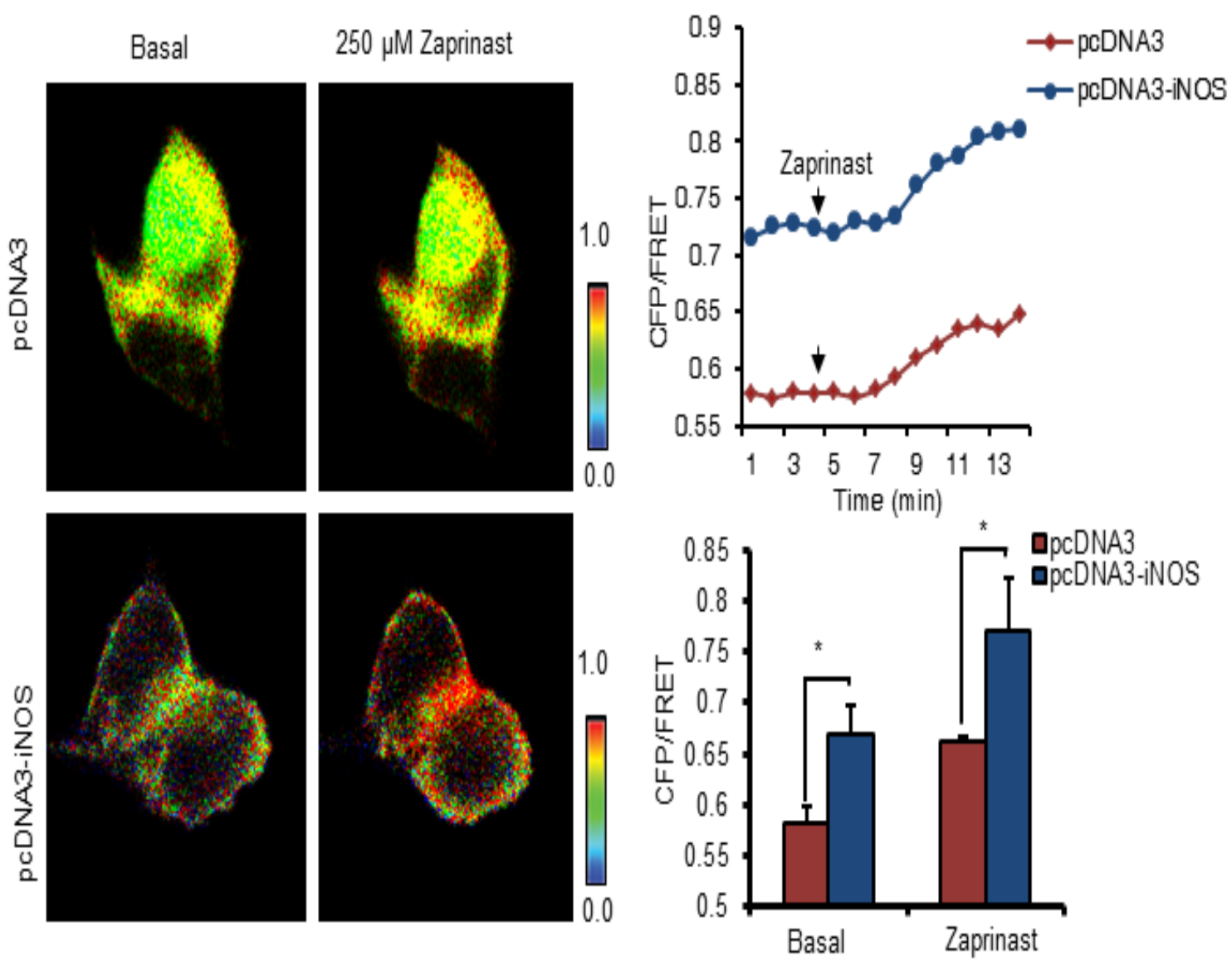

Figure 4-27. iNOS potentially constitutes cGMP microdomains at the plasma membrane

Representative pseudocolor images of CFP/FRET emission ratio before (time $=0$ ) and after adding $250 \mu \mathrm{M}$ zaprinast (time $=10 \mathrm{~min}$ ) in HEK-293 cells, transiently expressing membrane-bound cGMP sensor m-Cygnet 2.1, and transfected with pCDNA3 empty vector (upper panel) and pcDNA3-iNOS (lower panel). Representative line graph to show $\mathrm{CFP} / \mathrm{FRET}$ emission ratio change over time upon adding the agonist. Bar graph represents baseline and zaprinast-induced CFP/FRET emission ratio in empty vector vs. iNOS-transfected HEK293 cells. All data are mean $\pm \operatorname{SEM~}(\mathrm{n}=3) ;{ }^{*} P<0.05$ as determined using student's t-test. 

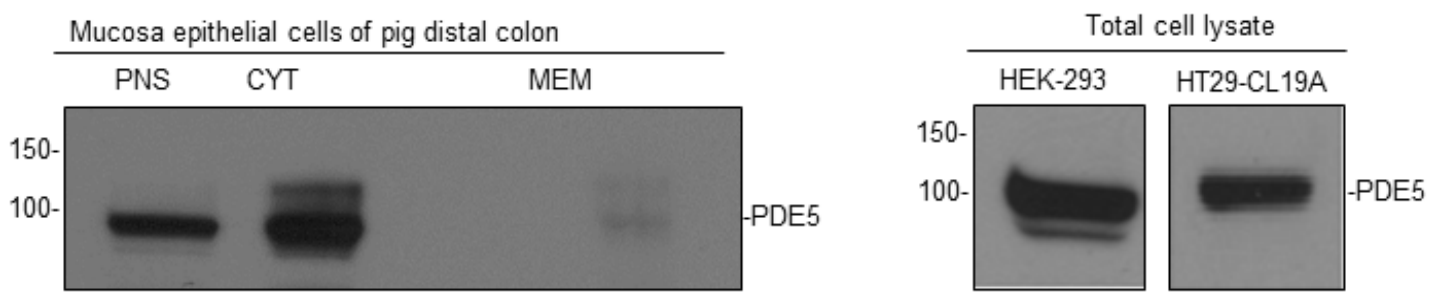

Figure 4-28. PDE5 expression in various cell systems

Western blot data to show PDE5 expression in post-nuclear supernatant (PNS), cytosol (CYT) and membrane (MEM) fractions of the pig distal colon tissue lysate (left) and, in HEK-293 and HT29-CL19A total cell lysate (right). 


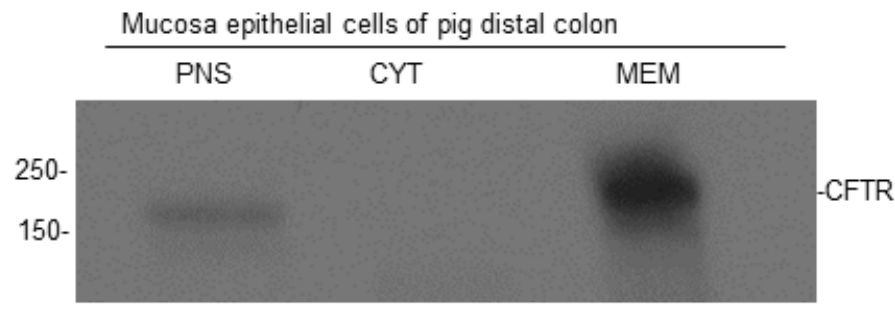

Figure 4-29. CFTR expression in colon mucosal epithelial cells

Western blot data to show CFTR expression in post-nuclear supernatant (PNS), cytosol (CYT) and membrane (MEM) fractions of the pig distal colon tissue lysate. 
macromolecular complex of CFTR and iNOS due to NHERF2 knockdown compared to that in the cells with intact NHERF2 (Figure 4-30A). CFTR function was not significantly different in HEK-293-S-Flag-CFTR and HEK-293-N-Flag-CFTR (Figure 4-30B).

\section{iNOS Potentiates CFTR Chloride Channel Function in a Cell Inflammatory Model}

PDE5-inh-dependent stimulation of CFTR currents has been demonstrated in lung airway cells (6). Viagra, a derivative of PDE5-inh, is used for treating male impotence, and, interestingly, patients who take the drug have diarrhea as a side-effect (134). We demonstrated that zaprinast increases CFTR-dependent short-circuit currents (8) in HT29-CL19A cells in a dose dependent manner (Figure 4-31A). We also measured iodide efflux rate in HT29-CL19A cells, and, consistent with the $I_{s c}$ measurement, zaprinast augmented CFTR function in the treated cells (Figure 4-31B). Forskolin a global activator of $\mathrm{AC}$ and CFTR chloride channel function was used as a positive control. It was observed that the effect seen with zaprinast on CFTR-function was small which may be accounted by the nature of how most of the PDEs function i.e. in a highly compartmentalized fashion. Therefore, high concentration of zaprinast was used for the studies. The nature of how PDEs usually function in cells has been reviewed in Chapter 1 . In order to elucidate the possibility of CFTR and iNOS physical and functional coupling at the plasma membrane, polarized HT-29CL19A cells were immunostained for CFTR and iNOS. HT29-CL19A cells endogenously express CFTR and iNOS was exogenously overexpressed in these cells. Immunofluorescence stained polarized cells demonstrated a high abundance of CFTR and iNOS at the apical plasma membrane and these two proteins are partially co-localized (Figure 4-32). Colon mucosal epithelial cells are known to be the major contributors of intestinal inflammation in IBD, so we investigated CFTR function in vitro in HT29-CL19A cells in response to an inflammatory challenge. These colon epithelial cells reportedly showed induced iNOS mRNA and increased nitrite levels in response to various cytokines (135). We simulated inflammatory conditions in HT29-CL19A cells by subjecting the cells to a cytokine mix or cytomix for $48 \mathrm{~h}$. The rationale of inducing inflammation in order to study outcomes of iNOS upregulation on CFTR function is to present a more relevant physiological model representative of in vivo inflammatory conditions. Subsequently, the relative change in iNOS-specific mRNA levels was determined using real-time PCR. iNOS expression increased almost 6-fold in response to the cytokines compared to the untreated controls (Figure 4-33). A small increase in the expression levels of CFTR and NHERF2 was also observed (Figure 4-33). We monitored $I_{s c}$ and measured iodide efflux rate in HT29CL19A cells pretreated with or without cytomix. After establishing basal currents or basal iodide secretion, cells were incubated with an inducer of iNOS, L- arginine $(1 \mathrm{mM})$, for $15 \mathrm{~min}$. CFTR was then activated with $250-400 \mu \mathrm{M}$ zaprinast. $\mathrm{CFTR}^{-}$channel function increased by 1.5- to 2-fold in cytomix-treated cells compared to the untreated cells (Figure 4-34). The difference of the effect on CFTR function observed between cytomix-treated and untreated sample was not dramatic which might be possible due to highly compartmentalized activation of CFTR within iNOS microdomains and it can be speculated that additional factors might be required in vivo in order to observe massive 

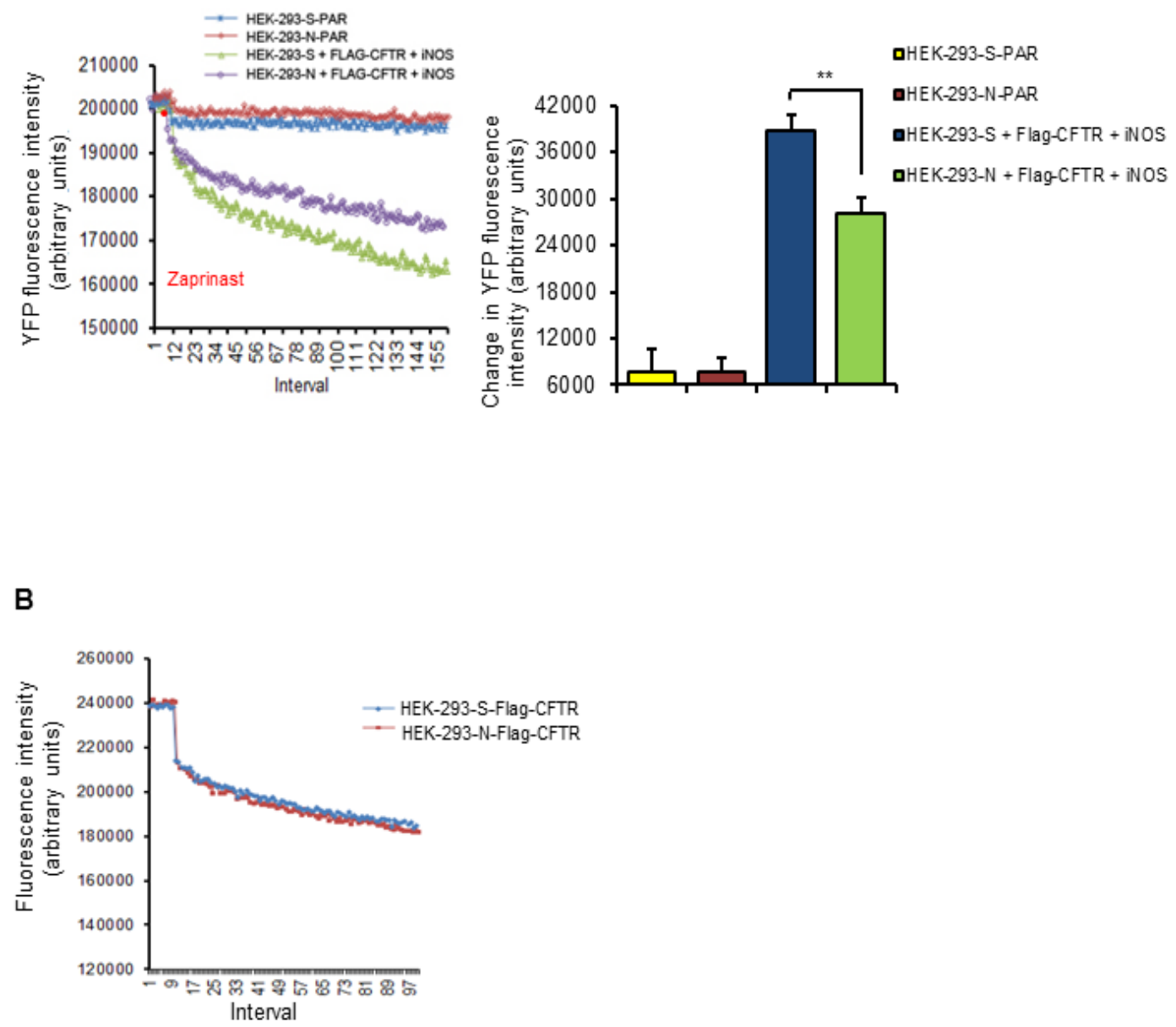

Figure 4-30. Knockdown of NHERF2 expression compromises CFTR-iNOS functional coupling

(A) Averaged representative traces of CFTR-mediated $\mathrm{I}^{-}$influx in HEK-293-S (scrambled siRNA) and HEK-293-N (NHERF2 siRNA) cells overexpressing Flag-CFTR and iNOS in response to $250 \mu \mathrm{M}$ zaprinast. PAR refers to the cells with no Flag-CFTR and iNOS. Quantification of the data represented as change in YFP fluorescence $(n=6)$. (B) Averaged representative traces of CFTR-mediated $\mathrm{I}^{-}$influx in HEK-293-S (scrambled siRNA) and HEK-293-N (NHERF2 siRNA) overexpressing Flag-CFTR in response to $250 \mu \mathrm{M}$ zaprinast $(\mathrm{n}=4-6)$. Data are mean $\pm \mathrm{SEM} ; * * P<0.01$ as determined using student's t-test. 
A

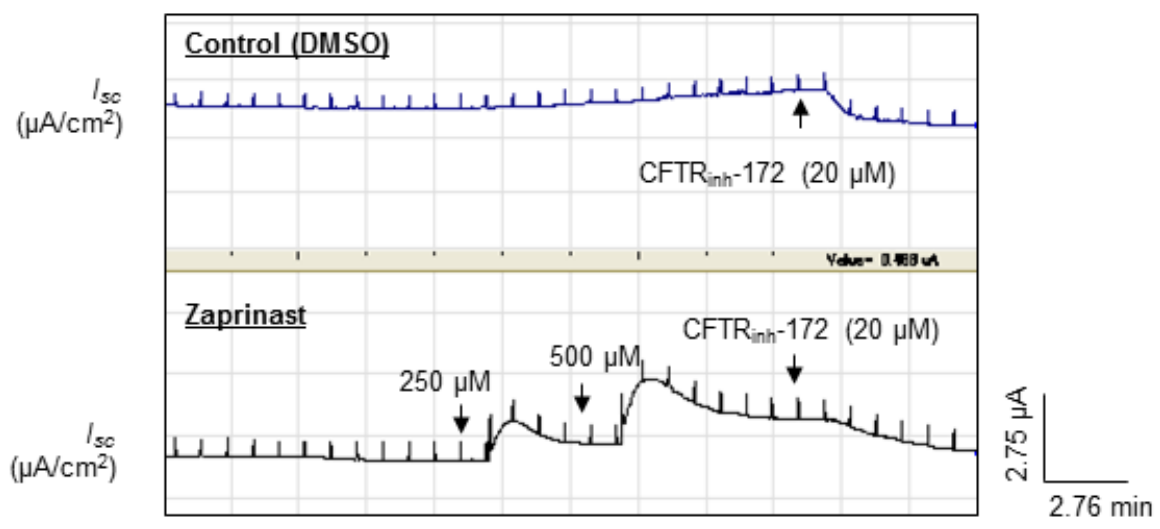

B

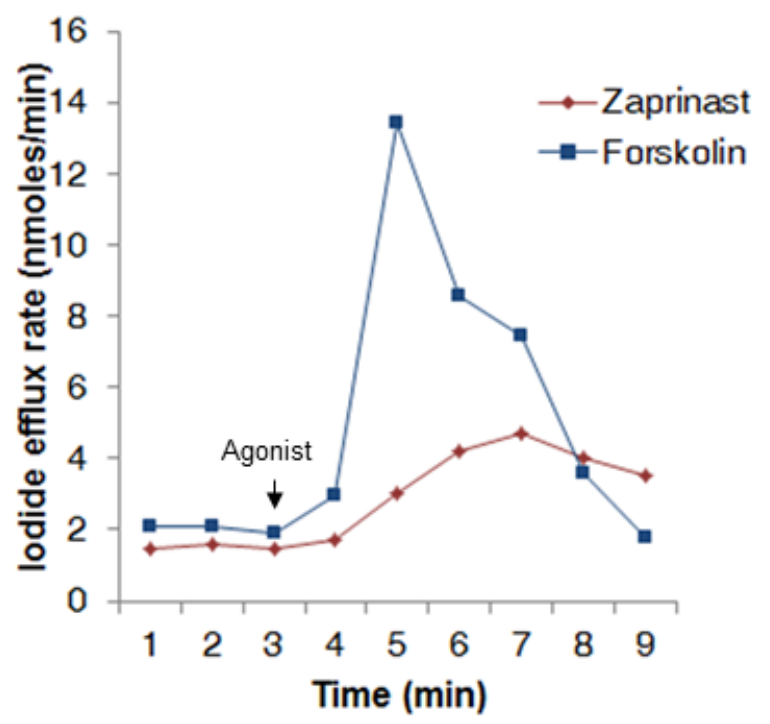

Figure 4-31. Zaprinast induced CFTR-chloride channel function

(A) Representative traces of CFTR-mediated short circuit currents $\left(I_{s c}\right)$ currents in polarized HT29-CL19A cells treated with various concentrations of zaprinast. CFTR $_{\text {inh }}{ }^{-}$ 172 was added to the apical side at the end of the experiment. (B) Line graph represents CFTR-mediated iodide efflux rate in HT29-CL19A cells activated with forskolin $(50 \mu \mathrm{M})$ or zaprinast $(250 \mu \mathrm{M})$. 

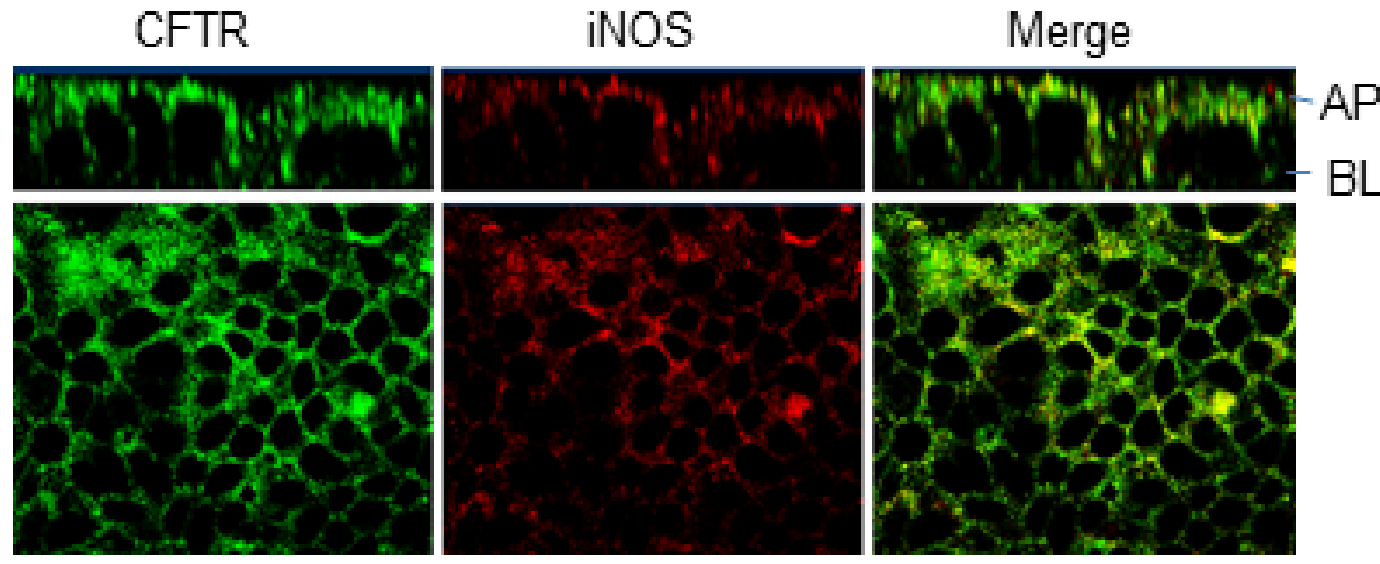

Figure 4-32. Localization of CFTR and iNOS in polarized HT29-CL19A cells 


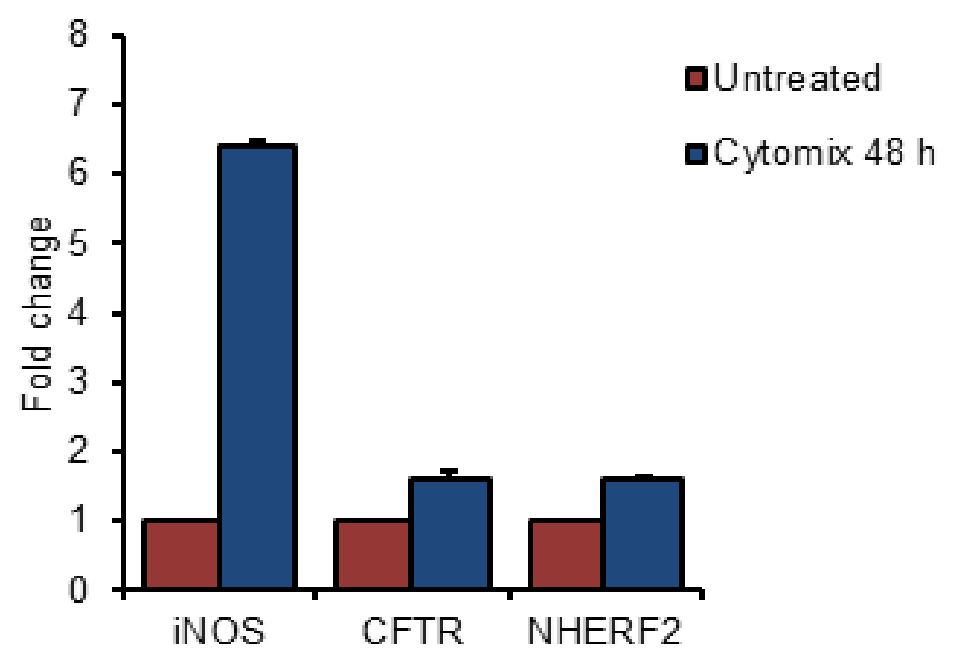

Figure 4-33. iNOS, CFTR and NHERF2 expression in cytomix challenged colon epithelial cells

The bar graph represents the fold change in iNOS, CFTR, NHERF2 transcripts by using real-time PCR (TaqMan probe-based method) in HT29-CL19A cells treated with cytomix for $48 \mathrm{~h}$. Data represent the mean $\pm \operatorname{SEM}(\mathrm{n}=3)$. 
A

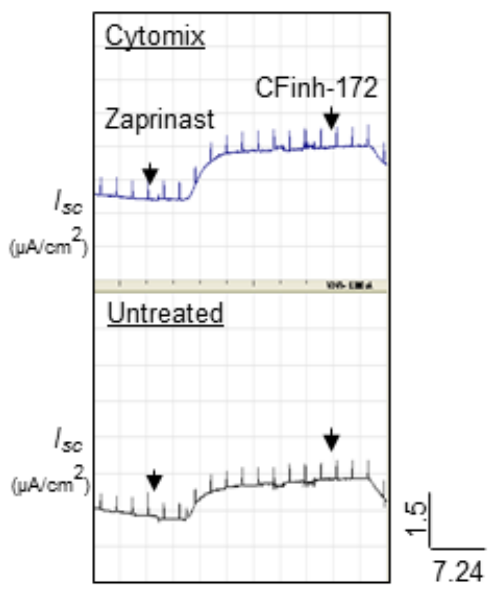

B

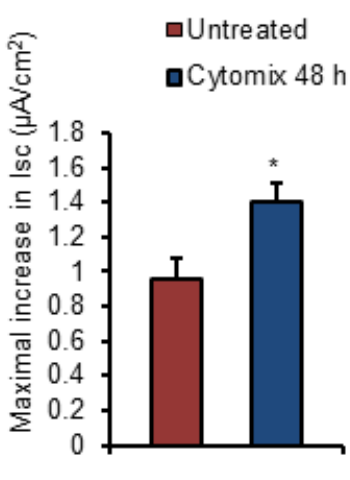

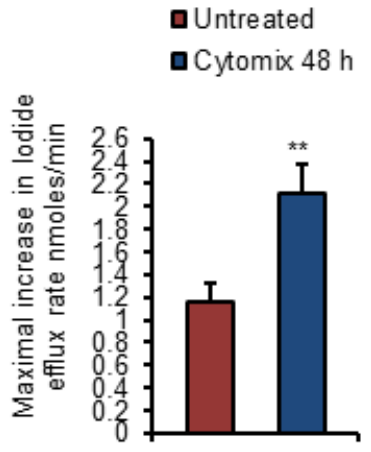

Figure 4-34. CFTR chloride channel function in cytomix-treated colon epithelial cells

(A) Representative trace of CFTR-mediated short circuit currents $\left(I_{s c}\right)$ in polarized HT29CL19A cells treated with cytomix for $48 \mathrm{~h}$ vs. untreated. Bar graph represents the maximal increase in $I_{s c}$ (cytomix treated vs. untreated cells) (n=3). (B) Bar-graph to show the maximal increase in iodide efflux rate to monitor CFTR activity in cytomix-treated vs. untreated HT29-CL19A cells $(\mathrm{n}=6)$. Data are mean $\pm \mathrm{SEM} ;{ }^{*} P<0.05,{ }^{*} P<0.01$ as determined using student's t-test. 
activation of CFTR chloride channel function. Also, an inflamed colon would present massive and highly active iNOS to regulate CFTR function which is challenging to achieve in a cell culture model.

Based on the abovementioned findings, it can be concluded that tight association of iNOS with the apical plasma membrane in IBD and polarized colon epithelial cells would form the basis of iNOS-dependent stimulation of CFTR in a highly compartmentalized fashion at the plasma membrane.

\section{Inhibition of CFTR and iNOS Activity Attenuates Diarrhea in DSS-induced Colitis}

No convincing data exists on the role of CFTR in IBD-associated diarrhea and many in vivo animal and human studies have emphasized that anion secretion may not be altered in colitis (103). With the major role of CFTR in regulation of fluid regulation across intestinal epithelial and pathogenesis of secretory diarrhea, it is considerably important to conclusively determine the role of CFTR in the pathogenesis of diarrhea in IBD (136). Ma et al. developed a highly specific thiozolidinone CFTR-inhibitor $\left(\right.$ CFTR $_{\text {inh }}$-172) which could significantly attenuate CTX-induced diarrhea in mice when administered intraperitoneally $(150 \mu \mathrm{g} / \mathrm{kg}$ for $6 \mathrm{~h})$ or orogavaged $(250 \mu \mathrm{g} / \mathrm{kg}$ for $6 \mathrm{~h})$ in these mice (137). More than 90\% reduction in the CTX-induced fluid secretion could be observed in mice orogavaged with the inhibitor. Based on these observations and highly specific action of CFTR inh -172 on CFTR, in order to conclusively determine if CFTR has a contribution in UC-associated diarrhea, colitis was developed in C57BL/6 mice using $5 \%$ DSS in the presence and absence of intraperitoneal administration of CFTR inh -172 $(150 \mu \mathrm{g} / \mathrm{kg}) 5$-day post DSS treatment. Preference was given to IP administration than orogavaging as the latter may have an effect on drinking water uptake by the mice. Control animals for the experiment included mice intraperitoneally administered with or without CFTR $_{\text {inh }}-172(150 \mu \mathrm{g} / \mathrm{kg})$ and normal autoclaved drinking water. Mice treated with DSS develop diarrhea and suffer from weight loss (117). The experimental layout of the experiment is represented and changes in the body weights of all the mice in each group were monitored every day in the span of the experiment (Figure 4-35). Administration of CFTR inhibitor was based on the appearance of symptoms in DSStreated animals. Animals started showing symptoms on the $4^{\text {th }}$ day of DSS-treatment. Mice were sacrificed on the $8^{\text {th }}$ day, and colons were removed for calculation of wet/dry weight, relative colon length, bleeding score and histological examination. Mice administered with CFTR $_{\text {inh- }}-172$ exhibited reduced weight loss $(25 \%)$ and fluid secretion compared to the untreated animals in the DSS group (Figures 4-35 and 4-36).

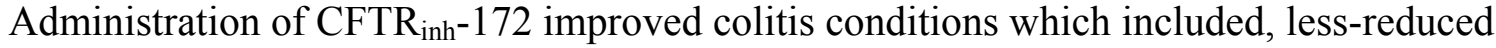
colon length, rectal bleeding score, wet/dry weight ratio compared to sole DSS treatment (Figure 4-37). In the absence of CFTR inh $^{-172}$, the wet/dry weight of colon was higher by $35 \%$ vs. when present (Figure 4-37). Interestingly, the histology score was not significantly altered with the inhibitor compared to the DSS-alone treatment (Figure 4-38). To the best our knowledge, this is the first study which sought to evaluate the role of CFTR in DSS-induced colitis and our data demonstrated that CFTR contributes significantly to DSS-induced diarrhea which can be extended to IBD-associated diarrhea. 

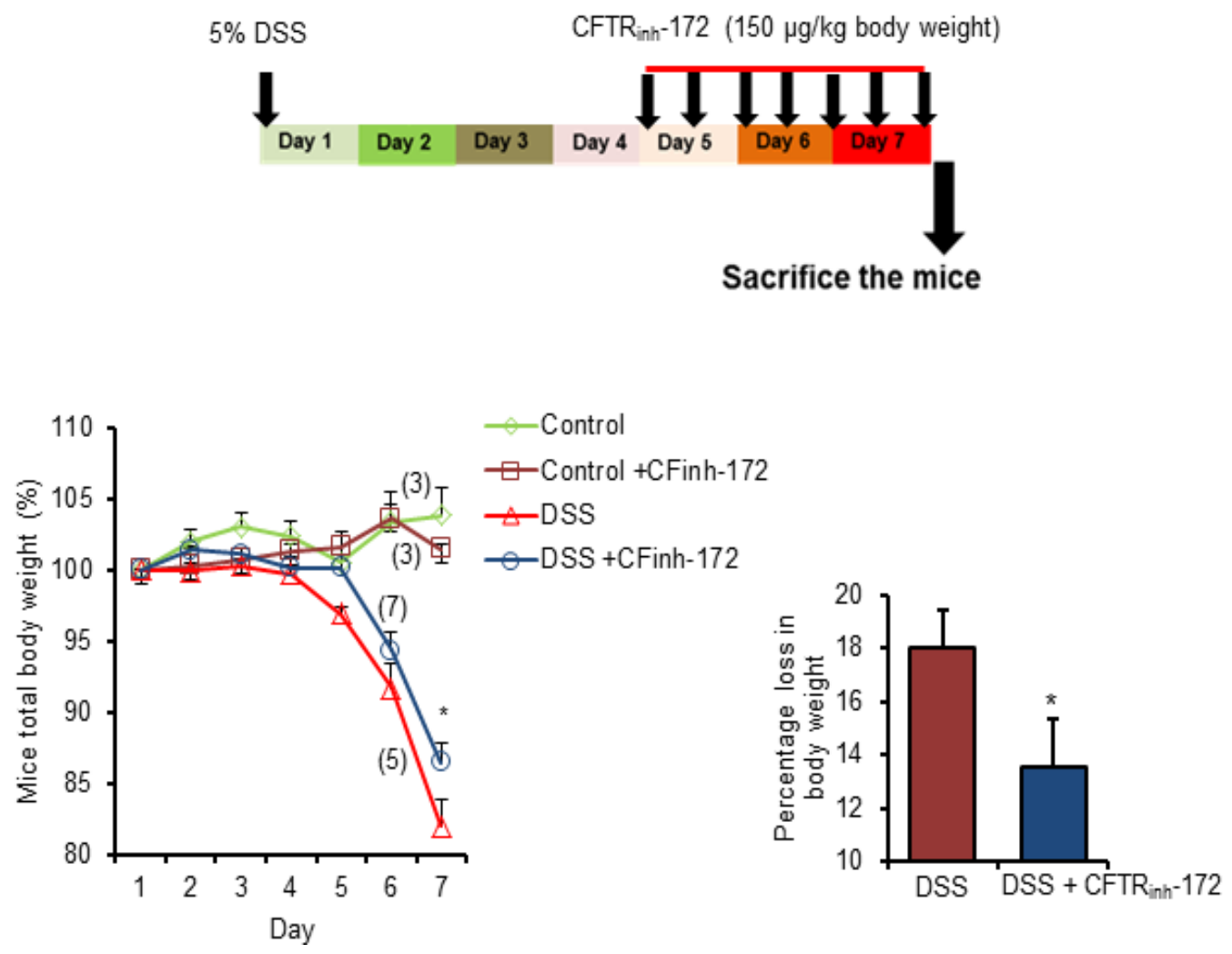

Figure 4-35. Inhibition of CFTR function causes partial protection against weight loss in DSS-colitis

A layout of the experiment involving CFTR $_{\text {inh }}-172$ treatment in DSS colitis model. Line graph shows the mouse total body weight measurements over a period of 7 days of the four-mice group; saline \pm DSS treatment and CFTR $_{\text {inh }}-172 \pm$ DSS. No DSS treatment groups had 3 mice each. DSS treatment group comprised of 5-8 mice. Bar-graph to show the total change in body weight of the mice on day 7 in $\mathrm{DSS} \pm \mathrm{CFTR}_{\text {inh }}-172$ experimental group. Data are mean $\pm \mathrm{SEM} ; * P<0.05$ as determined using student's t-test. 

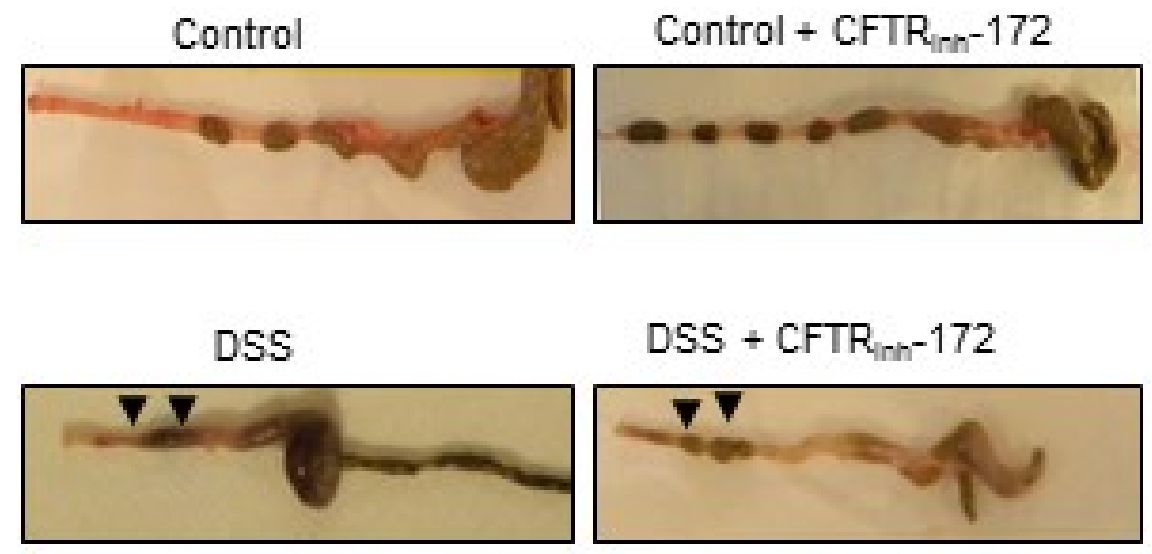

$$
\text { DSS + } \text { CFTR }_{\mathrm{inn}}-172
$$

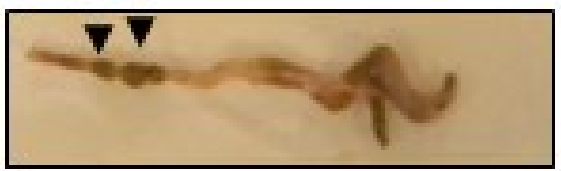

Figure 4-36. Inhibition of CFTR function attenuates fluid secretion in DSS-colitis

Representative separated mouse colons of the various treatment groups. Upper panel shows colon of the mice with no DSS $\pm \mathrm{CFTR}_{\text {inh }}-172$. Lower panel represents the colon isolated from mice in the DSS $\pm \mathrm{CFTR}_{\mathrm{inh}}-172$ treatment experimental group. 

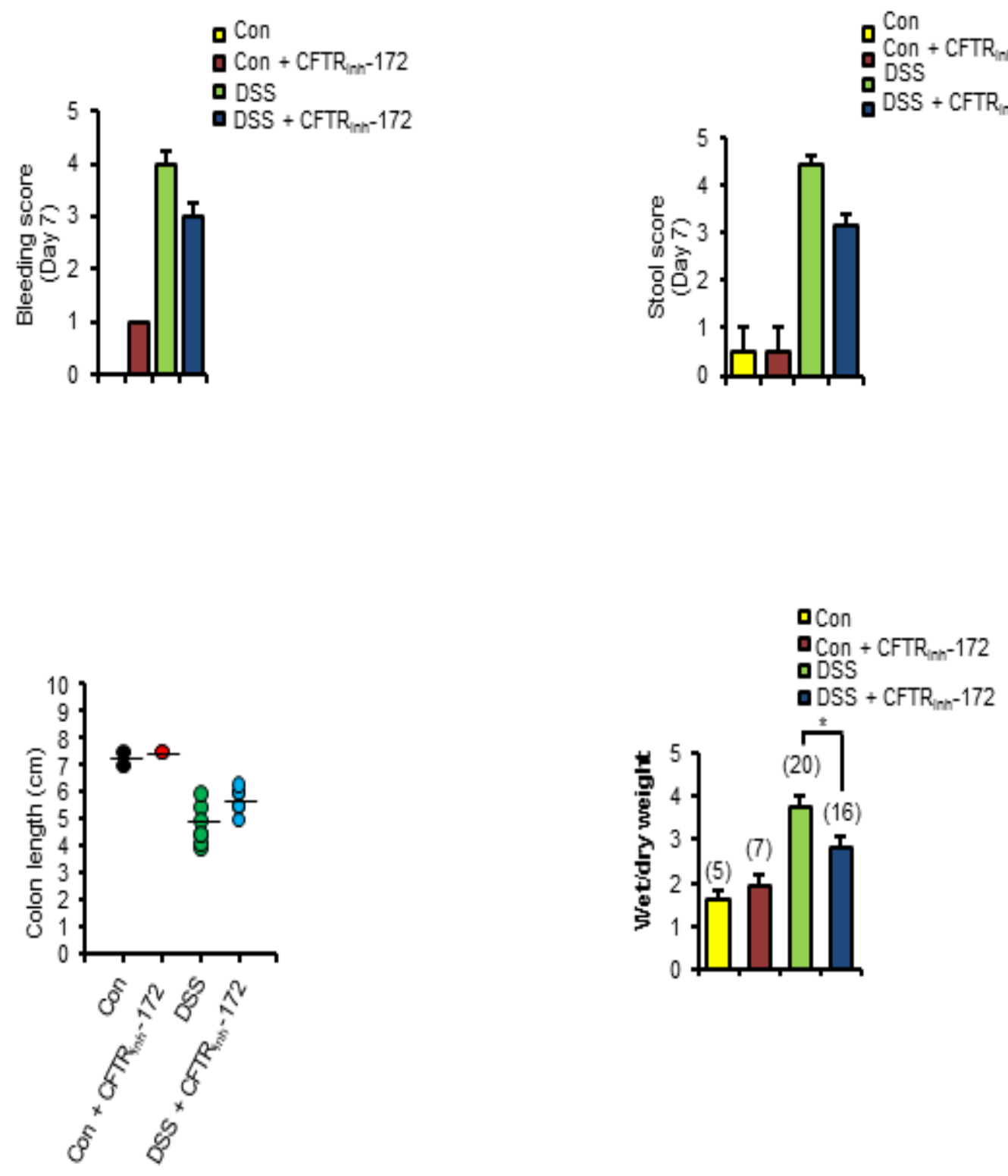

Figure 4-37. Inhibition of CFTR function causes partial protection in DSS-colitis

Bar-graphs show the assessments of various disease parameters of the DSS-induced colitis including bleeding and stool score and wet to dry weight ratio of the fecal pellets in the mice not receiving or receiving intraperitoneal injection of $\mathrm{CFTR}_{\text {inh }}-172$. X-Y scatter plot depicts the colon lengths of the mice in various groups. Control groups had 3 mice each. DSS treatment group comprised of 7-8 mice per group for CFTR inh-172 treatment. Data are mean $\pm \mathrm{SEM} ; * P<0.05$ as determined using student's t-test. 


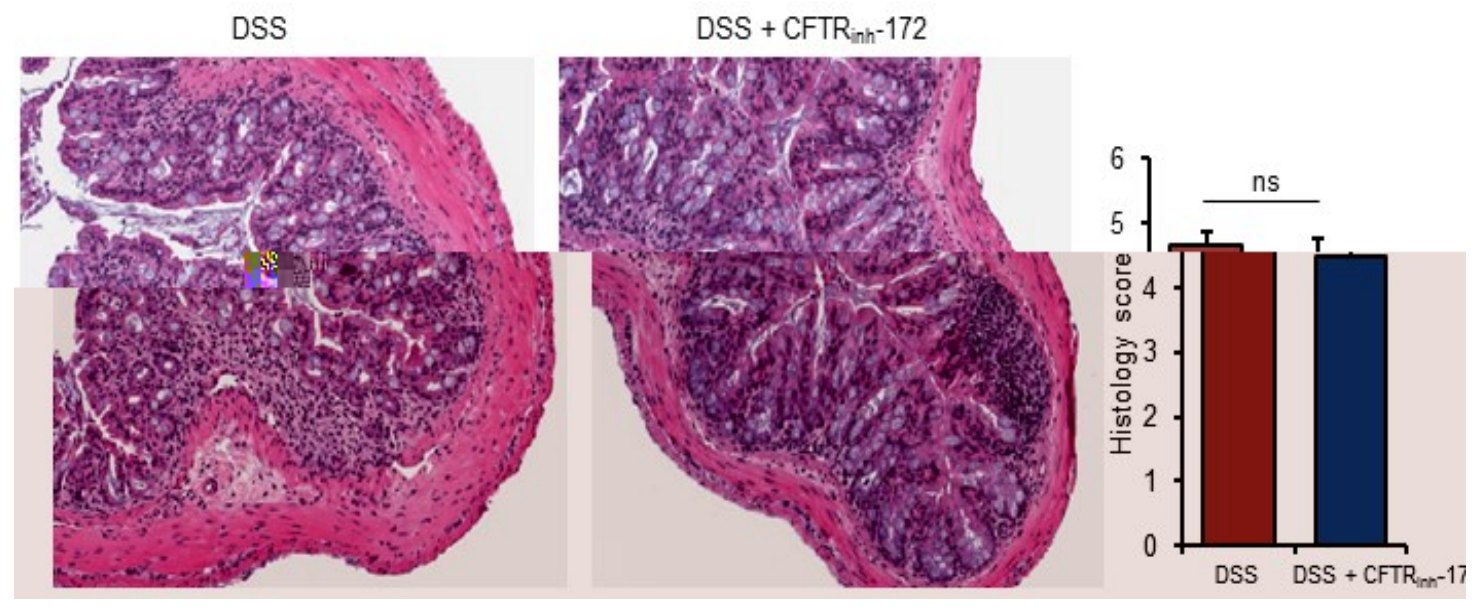

Figure 4-38. Histological scores are unaltered in the presence of CFTR inhibitor in DSS-colitis

H\&E stained colon sections of the mice from DSS \pm CFTR $_{\text {inh- }}-172$ treatment experimental group Bar graph represents the histology score of the colon sections. Data represent the mean \pm SEM $(n=6-12)$; ns= non-significant as determined using student's t-test. 
These observations have important clinical implications that CFTR inhibitors can have beneficial effects in the therapeutic intervention of IBD.

In order to elucidate the role of iNOS in DSS-induced diarrhea and colitis, we investigated the consequences of inhibiting iNOS activity in vivo in DSS-treated mice on fluid secretion in the colon. Previous studies have consistently determined that loss of iNOS causes reduced severity of DSS-induced colitis (138). Ablation of iNOS in TNBScolitis has generated equivocal results (75). However, it was concluded that the acute phase of colitis is critically affected by iNOS presence and activity in TNBS colitis and the absence of iNOS increases susceptibility to mucosal injury $(111,139)$. Based on these studies it can be understood that iNOS can have varied contribution in even closely related forms of inflammation. Therefore, generalization of an iNOS-dependent phenomenon must be precariously applied to other related gastrointestinal inflammatory disorders. Whether there exists an organ-specific phenotype of CFTR-iNOS macromolecular association in other organ-related inflammatory conditions needs to be further studied and evaluated.Studies involving use of NOS inhibitors have also generated mixed information and the inconsistencies largely stemmed from the narrow window of selectivity and specificity towards iNOS of these inhibitors and it has been inferred that certain NOS activity from nNOS might be essential for keeping the gut healthy $(75,112)$. Exogenous NO-donors could stimulate electrolyte secretion in guinea pig mucosa with the role of endogenous NO largely undetermined (140).

A recently published study documented the curative effects of iNOS inhibition using L-NIL on smoke induced pulmonary emphysema where it could prevent the development when co-administered with the smoke threat and could also reverse deterioration associated with the established emphysema (141). Other studies have reported specific action of L-NIL in the resolution of several other inflammatory profiles and in modulating inflammatory phenotypes in different models of intestinal inflammation. Considering the therapeutic effects of L-NIL on resolution of an inflammatory profile, we tested the effects of iNOS in DSS-colitis murine model and also, determined how endogenous NO generated from iNOS can alter fluid secretion in colon. L-NIL was administered intraperitoneally in a temporal variation in DSS-challenged wild-type mice. The temporal component of iNOS treatment is critical in view of suggested correlation between stage of iNOS inhibition and its outcome on disease activity index in experimental colitis models as mentioned previously (75). Fluid secretion was attenuated in L-NIL treated mice colons compared to the untreated (Figure 4-39). Animals treated with L-NIL one day after DSS-treatment appeared healthier and had significantly smaller weight loss $(16.3 \%)$ compared to the mice receiving intraperitoneal saline administration (Figure 4-39). Interestingly, mice which were treated with L-NIL three and five days after DSS-treatment trended to show improvement in the body weights compared to the control, however, it was less significant compared to the animals treated with L-NIL one day after colitis induction. No difference was observed in the animals which were administered with L-NIL or saline without DSS in the drinking water. Also, the disease activity parameters were significantly improved upon L-NIL treatment compared to the controls (Figure 4-40) which indicates the curative effects of L-NIL on DSS-induced colitis. The histological scores were improved 

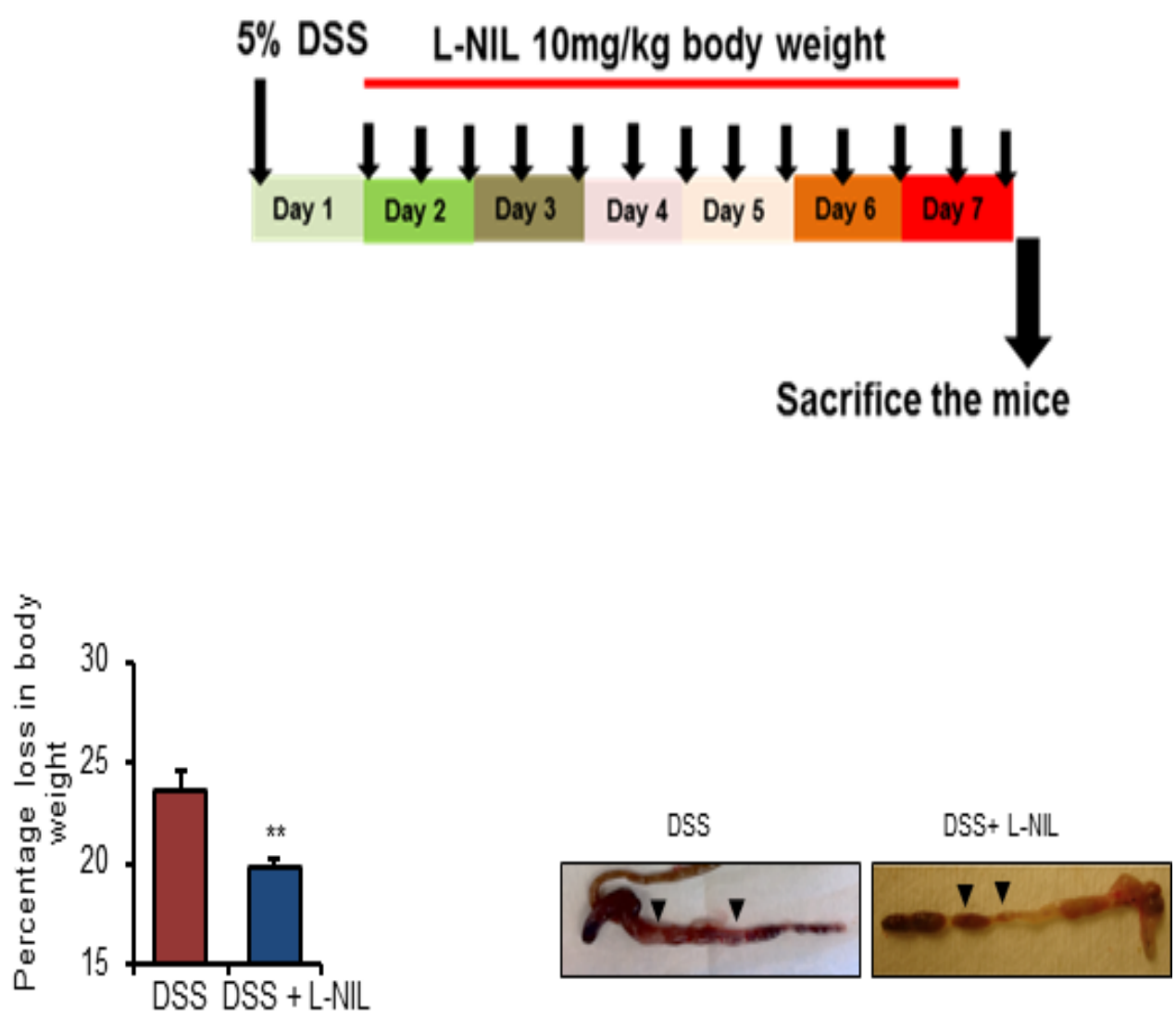

Figure 4-39. Inhibition of iNOS function causes partial protection against weight loss and attenuates fluid secretion in DSS-colitis

A layout of the experiment involving L-NIL treatment in DSS colitis model. Bar-graph to show the maximal change in body weight of the mice on day 7 in DSS \pm L-NIL experimental group. DSS treatment group comprised of 4-5 mice per group. The right panel represents the colon isolated from mice in the DSS \pm L-NIL treatment group. Arrows are marked to show the extent of fluid secretion in the colon. Data are mean \pm SEM; $* * P<0.01$ as determined using student's t-test. 

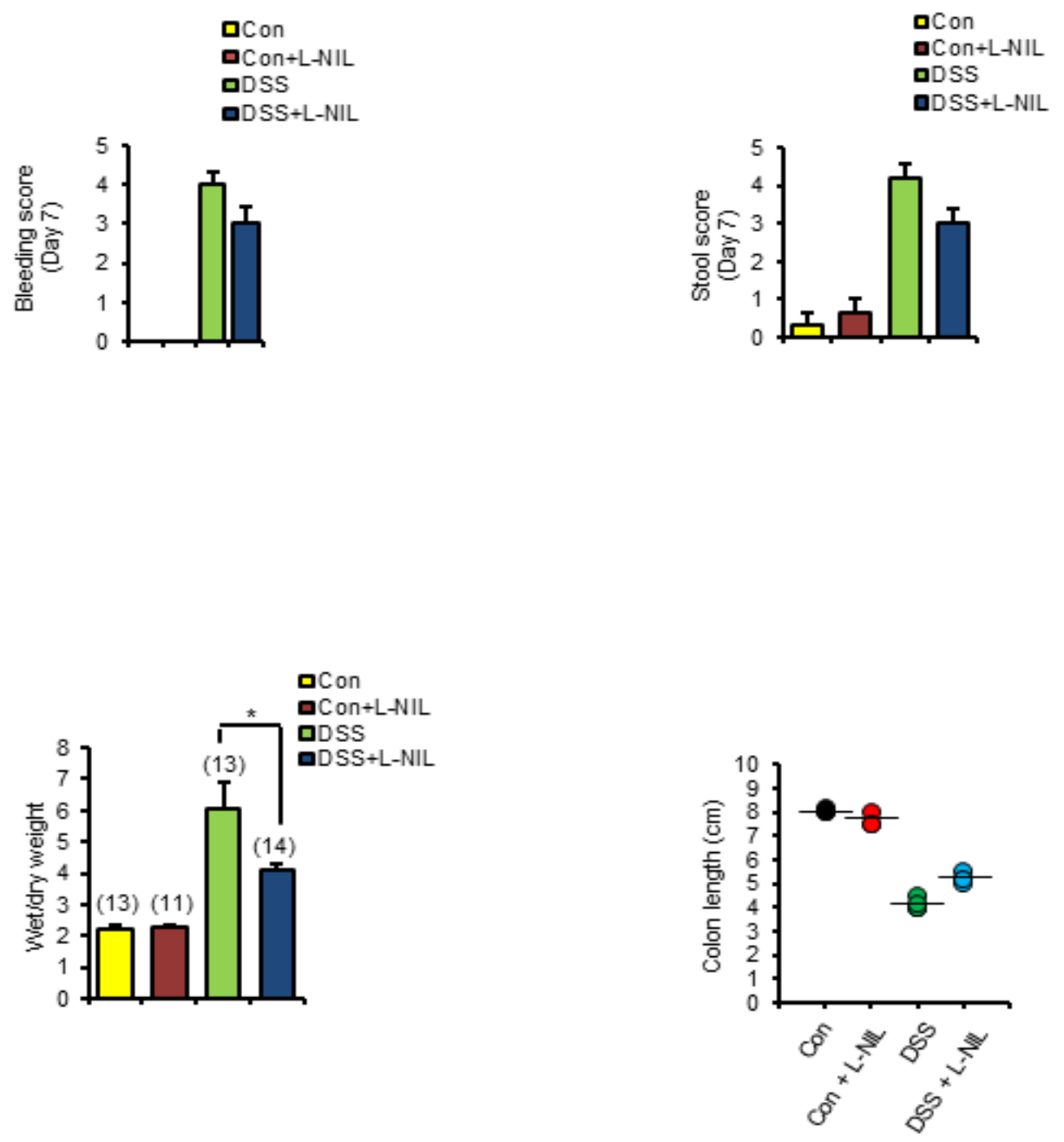

Figure 4-40. Inhibition of iNOS function causes partial protection against DSScolitis

The bar-graphs show the assessments of various disease parameters of the DSS-induced colitis including bleeding and stool score and wet to dry weight ratio of the fecal matter in the mice not receiving or receiving intraperitoneal injection of L-NIL. X-Y scatter plot depicts the colon lengths of the mice in various groups. Control groups had 3 mice each. The DSS treatment group comprised of 4-5 mice per group for L-NIL treatment. Data are mean $\pm \mathrm{SEM} ;{ }^{*} P<0.05$ as determined using student's t-test. 
with L-NIL treatment (Figure 4-41) Levels of NO in the homogenized colon tissue were quantified as nitrite using Griess reagent and normalized with the total protein concentration. DSS-exposed colon tissues were enriched with high amounts of NO compared to the unexposed tissue (Figure 4-42). The amount of NO was substantially reduced in the L-NIL treated animal group. cGMP levels were also determined using ELISA-based method and were found to be elevated in DSS-treated mice and decreased in response to L-NIL treatment (Figure 4-42). The data signifies that NO-cGMP pathway is actively operating in the DSS-colitis and is driven by up-regulated levels of iNOS. Also, effects observed with L-NIL treatment on DSS-colitis correctly correlate the contribution of endogenous NO contributed by highly active iNOS to increased fluid secretion in the affected mice.Also, it can be inferred that a right coordination of iNOS inhibition and its effect on other disease features of IBD than diarrhea can be explored as a potential therapeutic approach to control UC. Although, iNOS will be expressed by inflammatory cells also but secretory phenotype will be largely a contribution of epithelial cells.

\section{Diarrhea Is Attenuated in Nherf $2^{-/-}$Mice in DSS- and TNBS-induced Colitis}

Individuals suffering from IBD show severe diarrhea and weight loss. Given that potentiation of CFTR channel function happens in a compartmentalized manner through NHERF2-mediated formation of the CFTR-iNOS complex, we investigated the consequences of disrupting the compartmentalized response by uncoupling the proteininteractome as would be the case in Nherf $^{-/-}$mice. It is now being established that several macromolecular signaling complexes can become pathophysiological (46). Intuitively formation of macromolecular complexes is an evolutionary advantage for cell survival with the rationale that it prevents the dramatic functional effects of a protein and at the same time would contain the pathophysiological aspects of protein function. The current study emphasizes the fact the CFTR becomes pathophysiological in certain microdomains at the plasma membrane in IBD. In case of IBD, plasma membrane gets enriched with such microdomains and therefore, would simulate mass activated CFTR. The question arises how this mode of CFTR function regulation by iNOS in localized domains would be different from freely diffusing iNOS? The definitive advantage of compartmentalized regulation of CFTR is the selective and specific activation of CFTR and this also allows a major proportion of iNOS function to be channeled to CFTR regulation. Formation of a macromolecular complex also determines the nature of action of iNOS in the gut mucosa which would be predominantly vectorial. Our in vitro data established that the CFTR-NHERF2-iNOS macromolecular signaling complex increases CFTR function and that may have pathophysiological relevance to diarrhea in IBD. Next, we questioned what happens if the macromolecular complex is disrupted and if it can be beneficial for IBD therapeutics. DSS-induced colitis is the most widely used model that involves chemical-triggered manipulation of epithelial integrity/permeability and induces very reproducible severe acute colitis characterized by bloody diarrhea, ulcerations and granulocytes infiltrations (142). It is a particularly useful model to study the contribution of innate immune mechanisms of colitis and it gives the flexibility of inducing acute, chronic, or relapsing conditions of colitis with manipulations according to the 

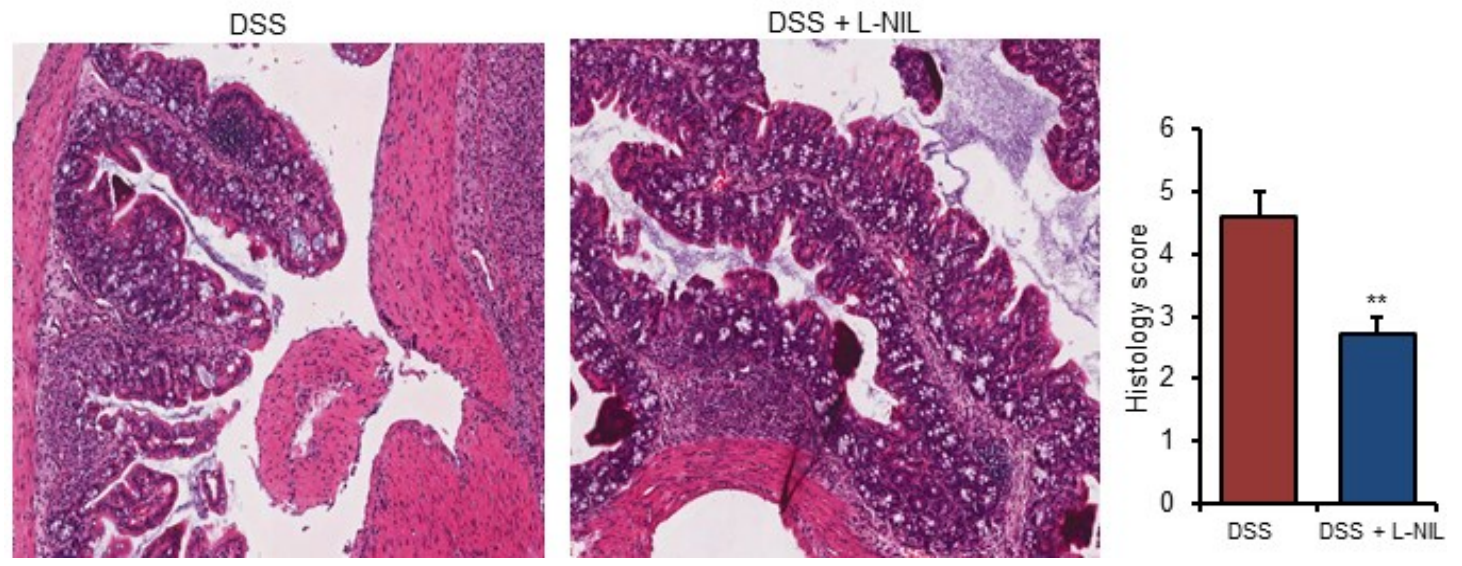

Figure 4-41. Inhibition of iNOS function improves histological scores in DSS-colitis

H\&E stained colon sections of the mice from DSS \pm L-NIL treatment experimental group Bar graph represents the histology score of the colon sections. Data represent the mean \pm $\operatorname{SEM}(\mathrm{n}=5-7) ; * * P<0.01$ as determined by student's t-test. 
A

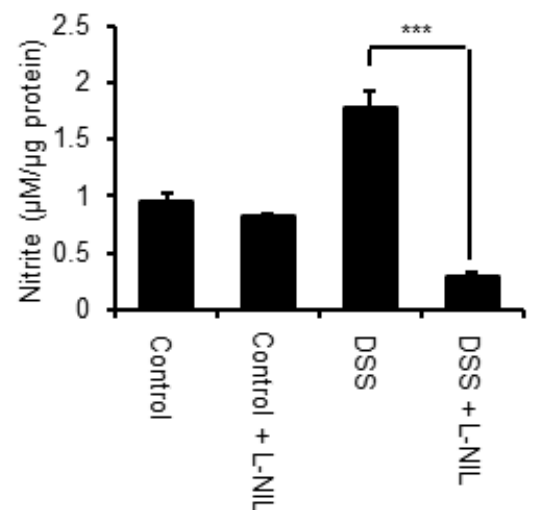

B

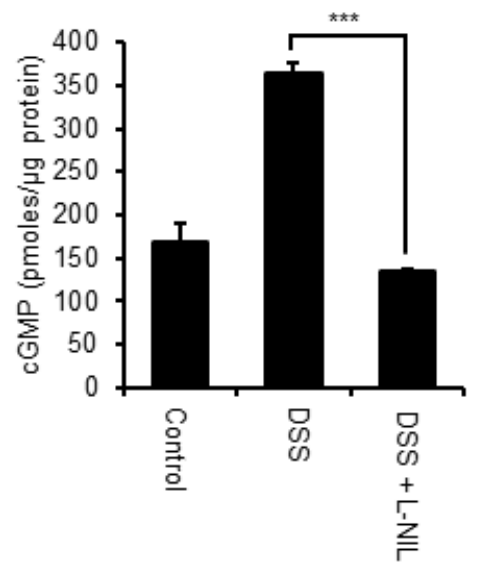

Figure 4-42. Inhibition of iNOS function affects NO-cGMP signaling in DSS-colitis

(A) Bar graph represents nitrite levels in colon tissues in response to DSS and DSS+LNIL treatment ( $\mathrm{n}=3$ colon tissue samples). (B) Bar graph represents cGMP levels in colon tissues in response to DSS and DSS+L-NIL treatment $(n=3-4$ colon tissue samples). All data are mean $\pm \mathrm{SEM} ;{ }^{* * *} P<0.001$ as determined using student's t-test. 
experimental needs. TNBS-colitis is the most widely used haptenating agent to initiate a CD-like intestinal inflammation in the colon and can recapitulate many of the events proposed to initiate and sustain human Crohn's disease (143).

We tested the differences in intestinal fluid secretion and weight loss, two indicators for secretory diarrhea, between $N h e r f 2^{-/}$and $N h e r f 2^{+/+}$mice in DSS- and TNBS-colitis models. Fluid secretion and the extent of diarrhea were assessed by calculating wet to dry weight ratio (wet/dry weight). Mice were weighed on a regular basis for 7 and 3 days in DSS- and TNBS-colitis models, respectively. Symptoms of colitis (rectal bleeding, weight loss) were observed from the $4^{\text {th }}$ day onwards after DSS treatment in all DSS-treated mice irrespective of the genetic background and similar bleeding score and reduction in colon length was observed on day 7 in Nherf $2^{-/-}$and Nherf $2^{+/+}$DSS-treated mice. No symptoms of colitis were observed in the untreated animals. TNBS was administered intra-rectally in ethanol based solution and mice demonstrated rapid weight loss on day 2 post-TNBS treatment characterizing the development of TNBS-induced colitis in both $N$ herf $2^{-/}$and Nherf $2^{+/+}$mice. Weight loss was assessed on the $7^{\text {th }}$ day of DSS treatment for all mice groups, and DSS-treated Nherf $2^{-/}$mice had significantly smaller maximal weight loss compared to DSS-treated Nherf $2^{+/+}$mice $\left(\sim 40 \%\right.$; Figure 4-43). On the $7^{\text {th }}$ day, colons were exteriorized, and wet/dry weights were calculated. Gross examination of the colons suggested relatively higher fluid secretion in Nherf $2^{+/+}$compared to Nherf $^{-/-}$mice in the DSS-treated groups, while no major difference was observed in the DSS-untreated group of animals (Figure 4-44). DSS-treated Nherf $2^{+/+}$mouse colon sections had $\sim 39 \%$ higher wet/dry weight compared to $\mathrm{Nherf}^{-/-}$mice, and the values in the untreated mouse groups were not significantly different (Figure 4-45). Similar to DSS-effects, Nherf2 ${ }^{-/}$were resistant to TNBS-induced colitis and demonstrated $65 \%$ lower maximal weight loss compared to Nherf $2^{+/+}$mice (Figure 4-46). Nherf $2^{-/-}$mice exhibited lower stool consistency score and wet to dry weight ratio was estimated to be lower by $42 \%$ in $N h e r f 2^{-/-}$than $N$ herf $2^{+/+}$mice in TNBS-colitis set-up (Figures 4-47 and 4-48). Similar histology scores were observed in Nherf $^{-/-}$and Nherf $2^{+/+}$mice in TNBS-colitis (Figure 4-49). These studies suggested that mice lacking NHERF2 were protected against DSS- and TNBS-induced diarrhea, and NHERF2 is required to mediate the functional coupling between CFTR and iNOS.

\section{Mass-Spectrometry of Purified iNOS}

The identity of the protein purified from Flag-iNOS expressing stable cell lines that indeed it is full-length Flag-iNOS protein was confirmed with mass spectrometry and also, there was a pool of data on the various proteins as potential interacting partners or modulators of iNOS function. Five major bands in iNOS immunoprecipitation lane were subjected to MS analysis as shown in Figure 4-50 and the results are shown in Tables 4-1 to 4-5. Information on the functions of immunoprecipitated proteins was obtained from http://www.uniprot.org. The Mowse score was calculated to opt out randomized interactions of low Mowse score and keep proteins with high Mowse score.

A Mowse score in peptide mass fingerprinting is a "similarity score" based on the 


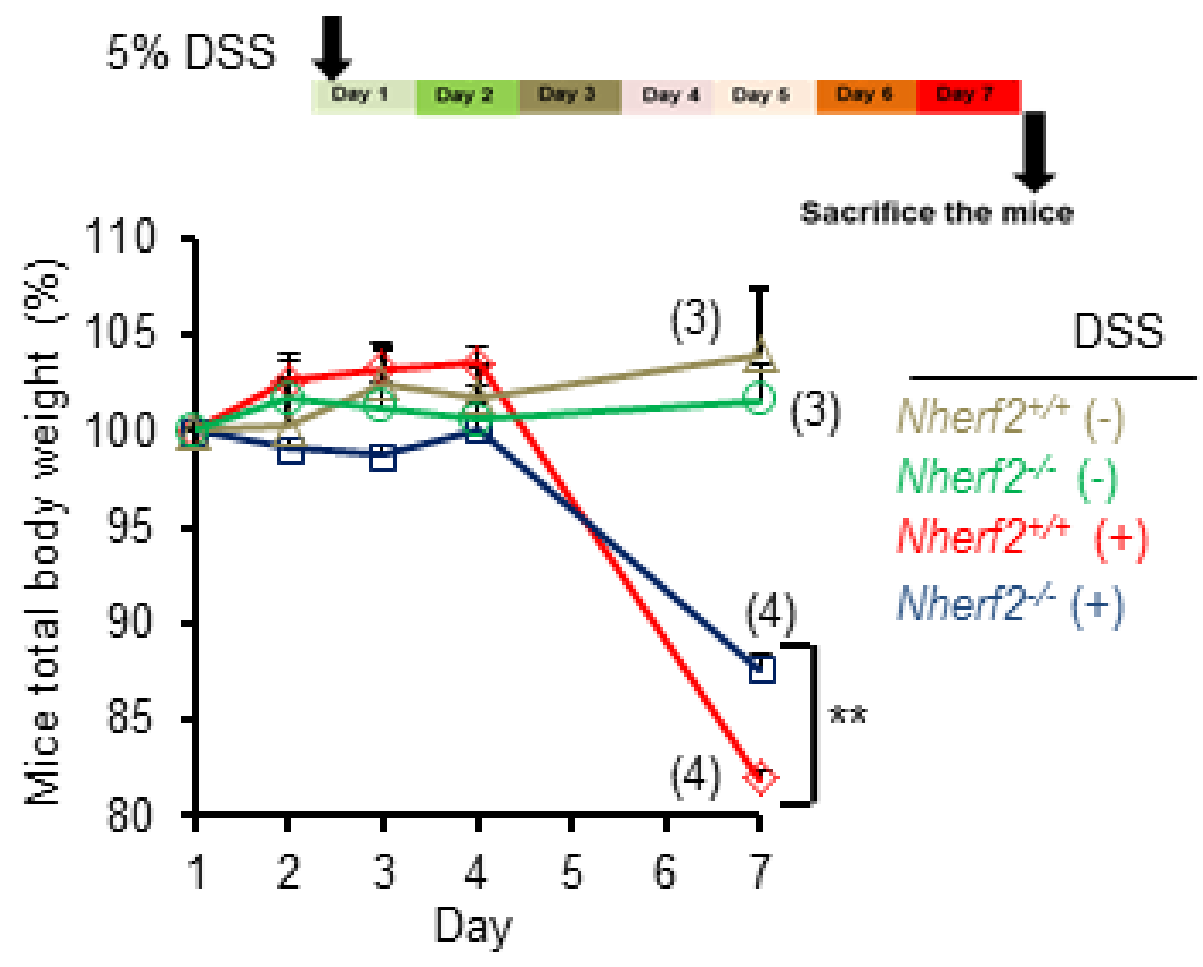

Figure 4-43. Nherf $^{-/-}$mice are partially protected against weight loss in DSS-colitis

An experimental layout of DSS treatment in $N$ herf $2^{+/+}$and $N h e r f 2^{-/-}$mice for a period of 7 days. Line graph shows the mouse total body weight measurements over a period of 7 days of the four-mice group; Nherf $2^{+/+} \pm$DSS treatment and Nherf $2^{-/} \pm$DSS treatment. Each group had 3-4 animals. Data are mean $\pm \mathrm{SEM}$; $* * P<0.01$ as determined using student's t-test. 

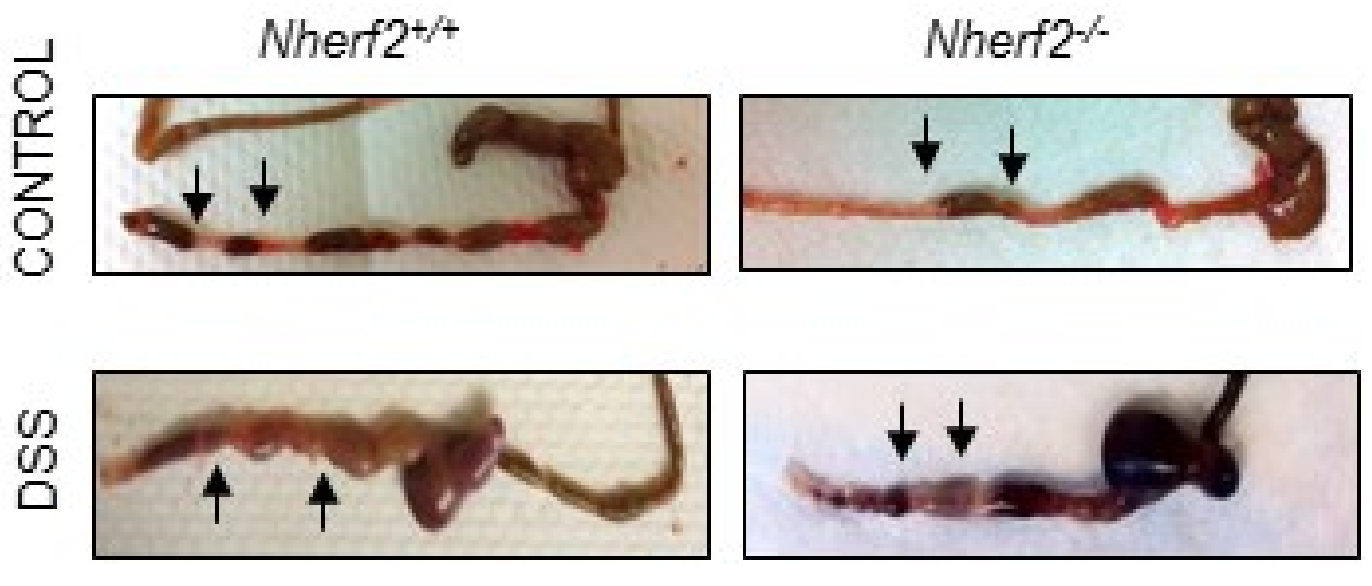

Figure 4-44. Nherf $2^{-/}$mice exhibit reduced fluid secretion than $N h e r f 2^{+/+}$mice in DSS-colitis

Representative exteriorized mouse colons of the various treatment groups. Upper panel and lower panel shows colon of Nherf $2^{+/+}$and Nherf $^{-/-}$mice with no DSS and with DSS, respectively. Arrows are marked to show the extent of fluid secretion in the colon. 

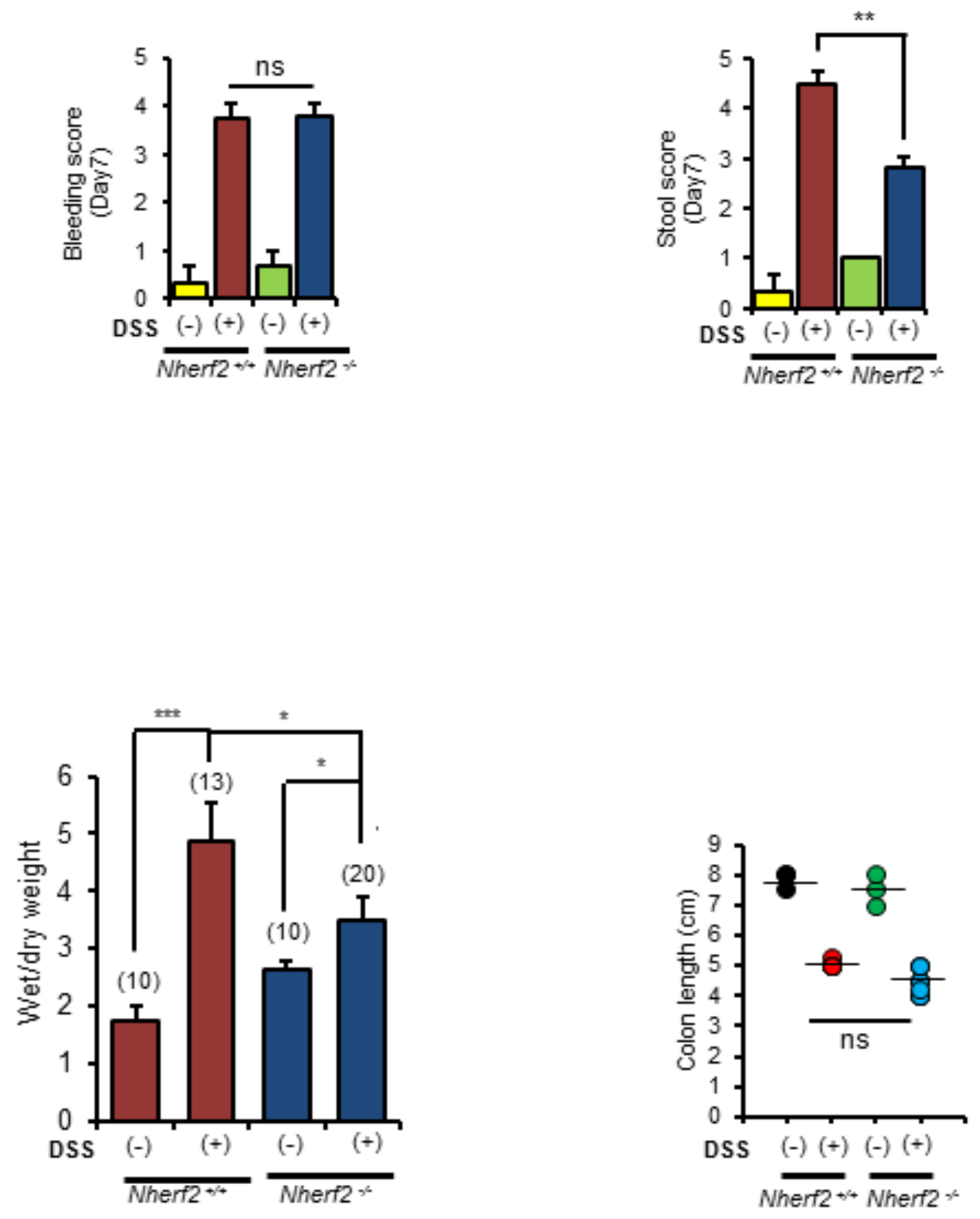

Figure 4-45. Nherf $^{-/-}$mice are partially protected against diarrhea in DSS-colitis

Bar-graphs show the assessments of various disease parameters of the DSS-induced colitis including bleeding and stool score and wet to dry weight ratio of the fecal pellets in the Nherf $2^{+/+}$and Nherf $2^{-/}$mice. X-Y scatter plot depicts the colon lengths of the mice in various groups. Control groups with no DSS had 3 mice each. DSS treatment group comprised of 8-10 mice per group. Data are mean $\pm \mathrm{SEM} * P<0.05, * * P<0.01, * * * P<$ $0.005, \mathrm{~ns}=$ non-significant as determined using student's t-test. 


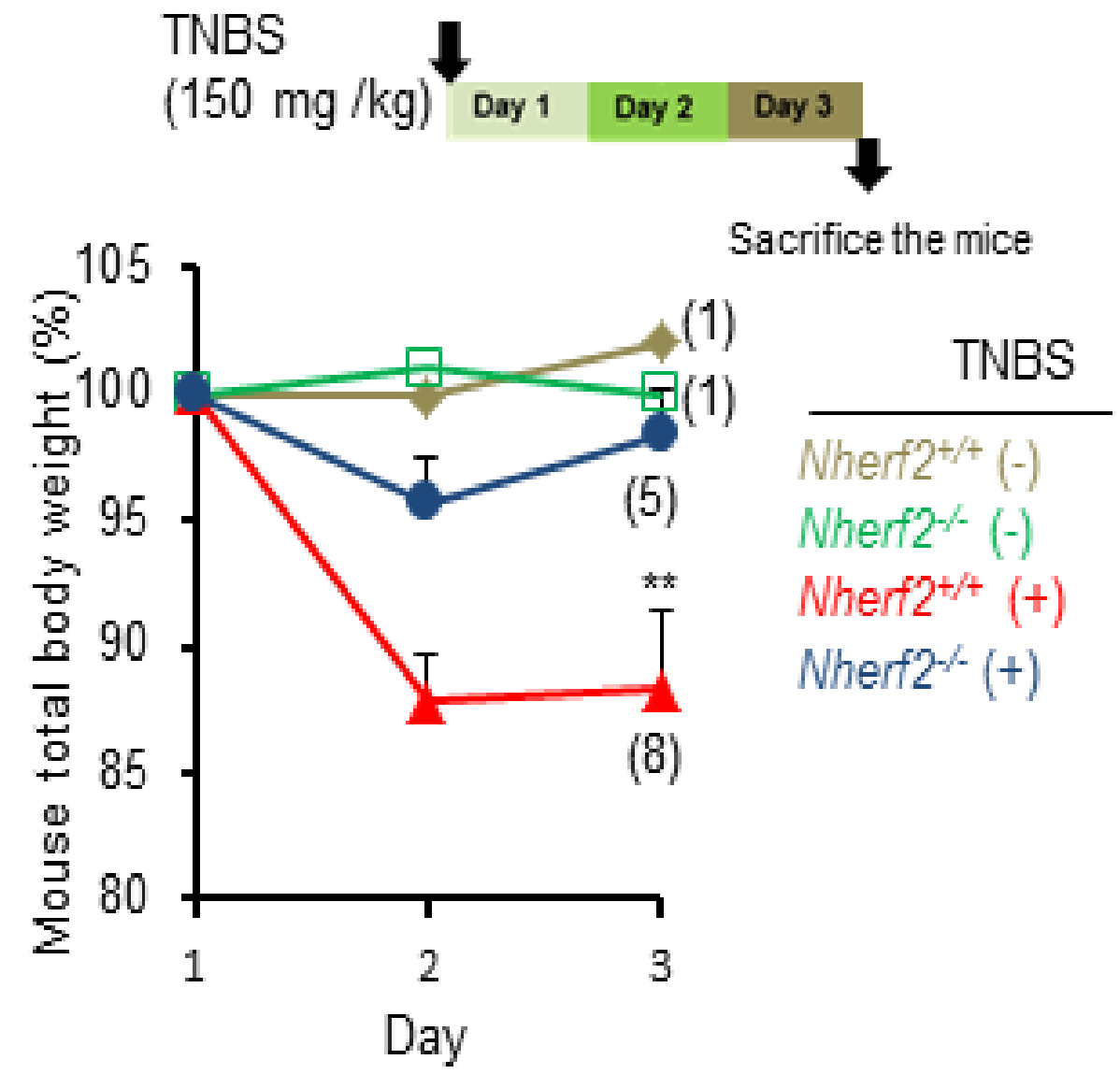

Figure 4-46. Nherf $^{-/-}$mice are partially protected against weight loss in TNBScolitis

Line graph shows the mouse total body weight measurements over a period of 3 days of the four-mice group; Nherf2 ${ }^{+/+} \pm$TNBS treatment and Nherf $2^{-/} \pm$TNBS treatment.

TNBS group had 5-8 animals. Data are mean \pm SEM; ${ }^{* *} P<0.01$ as determined using student's t-test. 

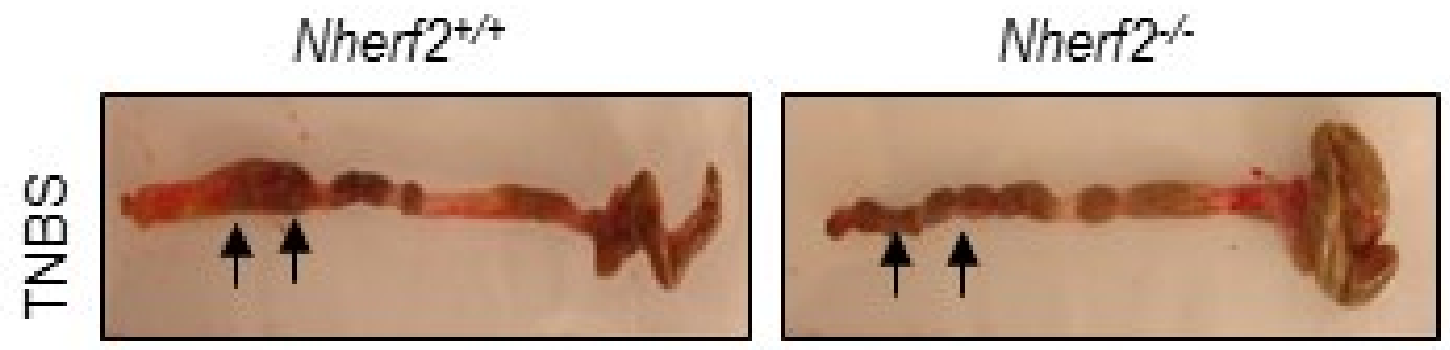

Figure 4-47. Nherf $2^{-/}$mice exhibit reduced fluid secretion than $N h e r f 2^{+/+}$mice in TNBS-colitis as depicted in the exteriorized colon sections 


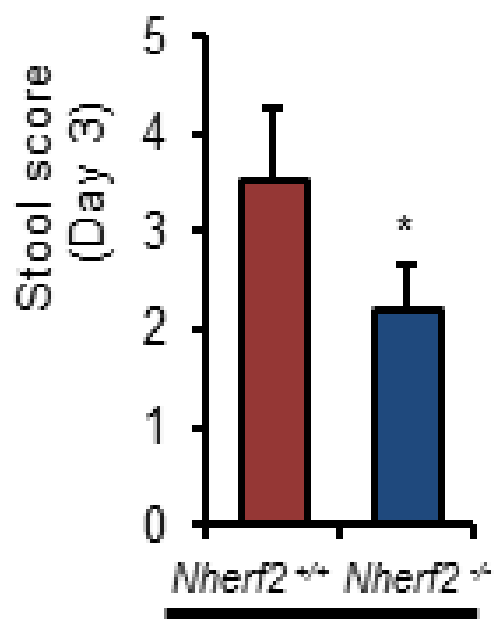

TNBS

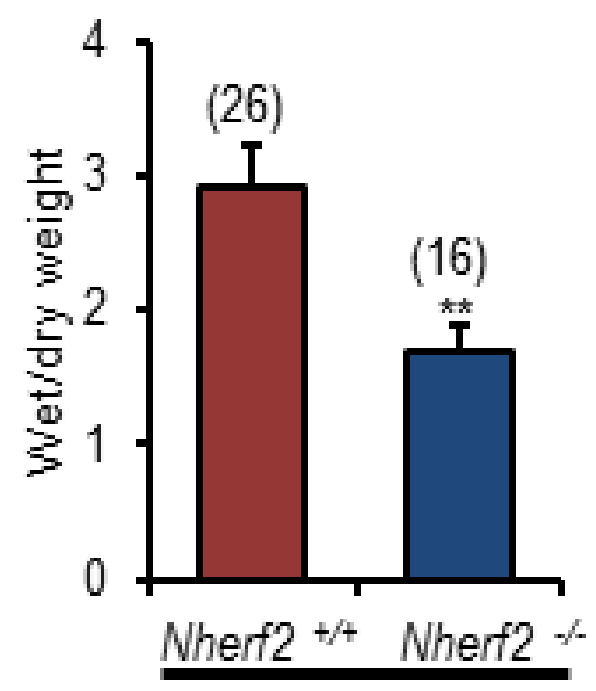

TNBS

Figure 4-48. Nherf $^{-/-}$mice are protected against diarrhea in TNBS-colitis

Bar-graphs show the measured stool score and wet to dry weight ratio of the fecal matter in the TNBS-treated $N h e r f 2^{+/+}$and $N h e r f 2^{-/}$mice. TNBS treatment group comprised of 8-10 mice per group. Data are mean $\pm \mathrm{SEM} ; * P<0.05$., $* * P<0.01$ as determined using student's t-test. 
TNBS-Nherf2 ${ }^{+/+}$

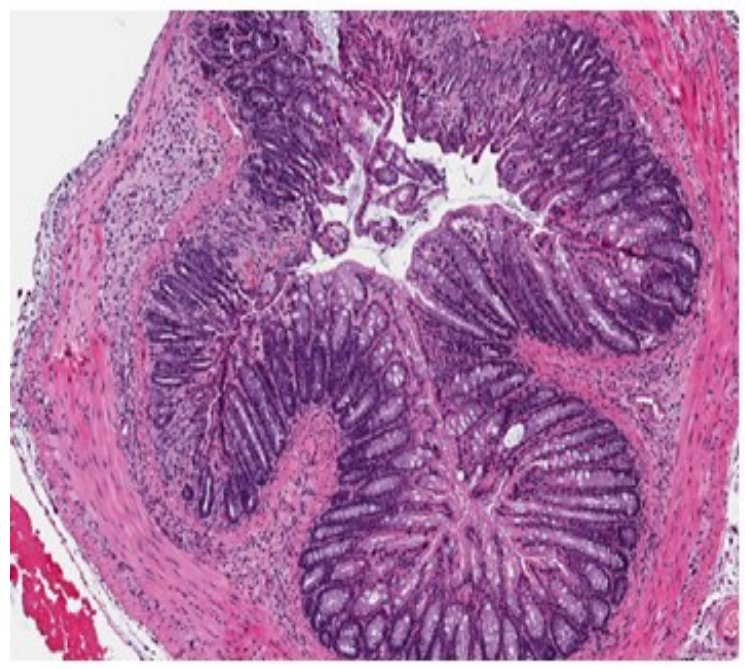

TNBS-Nherf2 $\%$

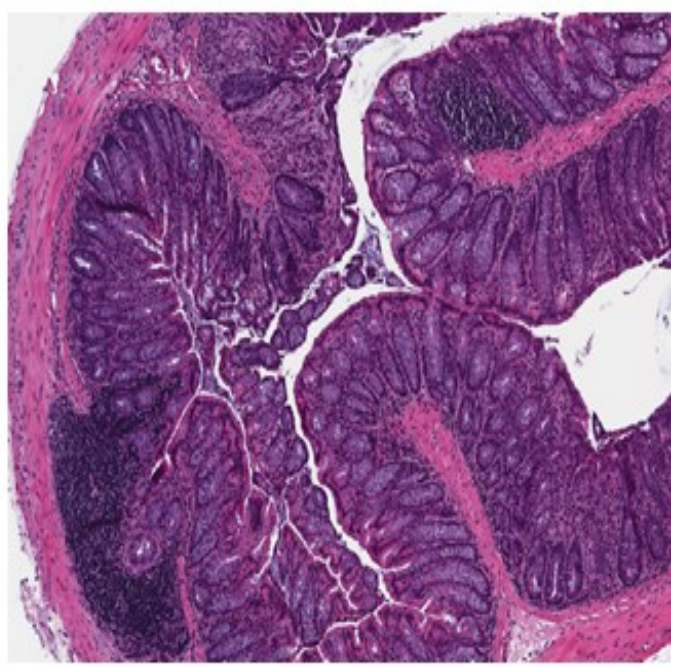

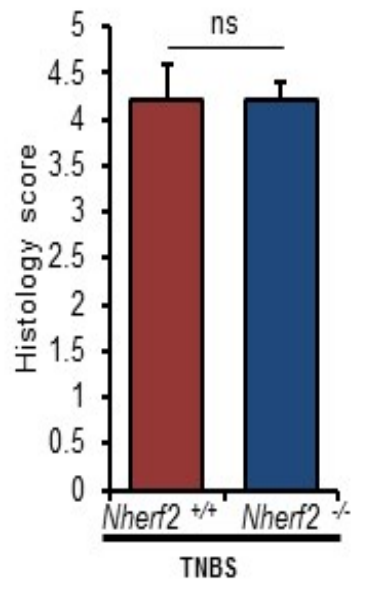

Figure 4-49. Histological scores are not different in $\mathrm{Nherf}^{-/-}$mice than $\mathrm{Nherf}^{+/+}$ mice in TNBS-colitis

H\&E stained colon sections of the TNBS treated Nherf $2^{+/+}$and Nherf2 $2^{-/}$mice. Bar graph represents the histology score of the colon sections. Data represent the mean $\pm \mathrm{SEM}$ $(n=5-10) ; n s=$ non-significant as determined using student's t-test. 


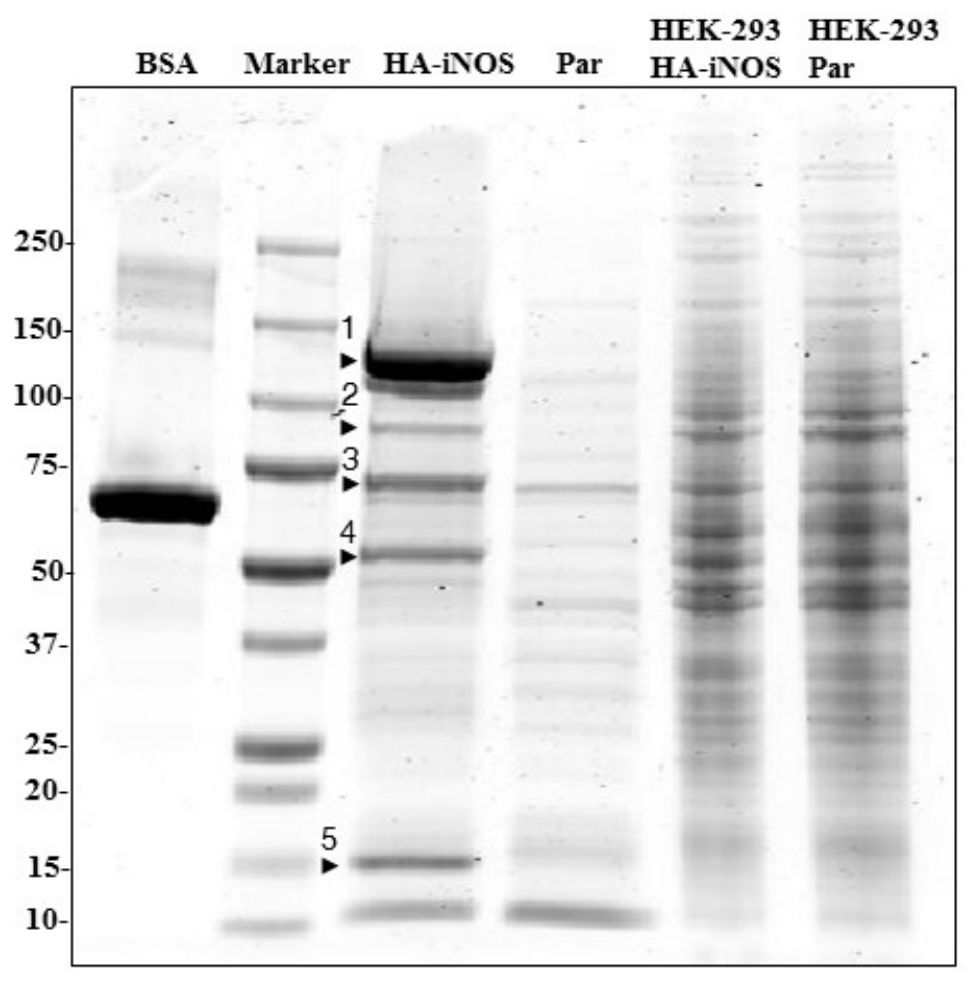

Figure 4-50. Immunoprecipitation of full-length HA-iNOS for mass-spectrometric analysis of bands 1-5 as marked 
Table 4-1. Mass-spectrometric analysis of immunopurified iNOS (Band 1)

\begin{tabular}{lllll}
\hline Protein & & Mowse score & & Function \\
\cline { 1 - 1 } $\begin{array}{l}\text { Nitric oxide synthase, } \\
\text { inducible NOS2 }\end{array}$ & & 12890 & & NO-synthesis \\
$\begin{array}{l}\text { Serine/threonine-protein } \\
\text { kinase Chk1 }\end{array}$ & 80 & & $\begin{array}{l}\text { Cell cycle, DNA damage, DNA } \\
\text { repair }\end{array}$ \\
\hline
\end{tabular}


Table 4-2. Mass-spectrometric analysis of immunopurified iNOS (Band 2)

\begin{tabular}{|c|c|c|}
\hline Protein & Mowse score & Function \\
\hline $\begin{array}{l}\text { Heat shock protein HSP } \\
\text { 90-beta }\end{array}$ & 2728 & Chaperone \\
\hline $\begin{array}{l}\text { Heat shock protein HSP } \\
\text { 90-alpha }\end{array}$ & 2122 & Chaperone \\
\hline $\begin{array}{l}\text { Arf-GAP with coiled- } \\
\text { coil, ANK repeat and PH } \\
\text { domain-containing } \\
\text { protein } 2\end{array}$ & 818 & GTPase-activating protein \\
\hline $\begin{array}{l}\text { Putative heat shock } \\
\text { protein HSP 90-beta } 2\end{array}$ & 725 & Chaperone \\
\hline $\begin{array}{l}\text { Heat shock cognate } 71 \\
\mathrm{kDa} \text { protein }\end{array}$ & 626 & Chaperone \\
\hline $\begin{array}{l}\text { Putative heat shock } \\
\text { protein HSP 90-beta } 4\end{array}$ & 415 & Chaperone \\
\hline $\begin{array}{l}\text { Interleukin enhancer- } \\
\text { binding factor } 3\end{array}$ & 367 & Transcription \\
\hline $\begin{array}{l}\text { Heat shock } 70 \mathrm{kDa} \\
\text { protein } 6\end{array}$ & 296 & Chaperone \\
\hline Pantothenate kinase 4 & 241 & Co-A synthesis \\
\hline $\begin{array}{l}\text { Heat shock protein } 75 \\
\mathrm{kDa} \text {, mitochondrial }\end{array}$ & 185 & Chaperone \\
\hline Endoplasmin & 158 & $\begin{array}{l}\text { Chaperone, proper folding of Toll- } \\
\text { like receptors, ER-associated } \\
\text { degradation }\end{array}$ \\
\hline $\begin{array}{l}\text { A-kinase anchor protein } \\
\text { 8-like }\end{array}$ & 135 & Helicase A binding protein \\
\hline $\begin{array}{l}\text { Protein arginine } \mathrm{N}- \\
\text { methyltransferase } 5\end{array}$ & 123 & Protein methylation \\
\hline $\begin{array}{l}\text { Golgi-specific brefeldin } \\
\text { A-resistance guanine }\end{array}$ & 121 & $\begin{array}{l}\text { Post-Golgi vesicle-mediated } \\
\text { transport and regulation of Arf- } \\
\text { GAP }\end{array}$ \\
\hline $\begin{array}{l}\text { E3 UFM1-protein ligase } \\
1\end{array}$ & 115 & $\begin{array}{l}\text { Negative regulation of NF-kappaB } \\
\text { transcription factor activity and } \\
\text { possibly acts as tumor suppressor }\end{array}$ \\
\hline $\begin{array}{l}78 \mathrm{kDa} \text { glucose-regulated } \\
\text { protein }\end{array}$ & 107 & $\begin{array}{l}\text { Chaperone and is involved in the } \\
\text { activation of unfolded protein } \\
\text { response in response to glucose } \\
\text { starvation }\end{array}$ \\
\hline $\begin{array}{l}\text { Probable Xaa-Pro } \\
\text { aminopeptidase } 3\end{array}$ & 99 & $\begin{array}{l}\text { Protein processing and proteolysis, } \\
\text { implicated in polycystic kidney } \\
\text { disorders }\end{array}$ \\
\hline
\end{tabular}


Table 4-2. Continued

\begin{tabular}{|c|c|c|}
\hline Protein & Mowse score & Function \\
\hline $\begin{array}{l}\text { Mannosyl- } \\
\text { oligosaccharide } \\
\text { glucosidase }\end{array}$ & 87 & $\begin{array}{l}\text { Protein N-linked glycosylation via } \\
\text { asparagine }\end{array}$ \\
\hline Catenin beta- 1 & 80 & $\begin{array}{l}\text { Component of Wnt-pathway and } \\
\text { also implicated in multiple-organ } \\
\text { cancer }\end{array}$ \\
\hline $\begin{array}{l}\text { Acyl-CoA } \\
\text { dehydrogenase family } \\
\text { member } 11\end{array}$ & 78 & Fatty-acid metabolism \\
\hline Junction plakoglobin & 75 & $\begin{array}{l}\text { Adherens junction organization } \\
\text { and cell migration }\end{array}$ \\
\hline $\begin{array}{l}\text { Serine/threonine-protein } \\
\text { kinase TBK } 1\end{array}$ & 73 & $\begin{array}{l}\text { Triggers I-kappaB kinase/NF- } \\
\text { kappaB cascade and regulator of } \\
\text { innate immune response }\end{array}$ \\
\hline Sulfhydryl oxidase 2 & 71 & $\begin{array}{l}\text { Disulfide bond formation and } \\
\text { synthesis of } \mathrm{H}_{2} \mathrm{O}_{2}\end{array}$ \\
\hline $\begin{array}{l}\text { Carnitine O- } \\
\text { palmitoyltransferase } 1 \text {, } \\
\text { liver isoform }\end{array}$ & 68 & Lipid metabolism \\
\hline $\begin{array}{l}\text { Melanoma-associated } \\
\text { antigen D1 }\end{array}$ & 67 & Ubiquitination and apoptosis \\
\hline Cullin-5 & 65 & $\begin{array}{l}\text { Ubiquitination and response to } \\
\text { osmotic stress }\end{array}$ \\
\hline $\begin{array}{l}\text { Pyridoxal-dependent } \\
\text { decarboxylase domain- } \\
\text { containing protein } 20\end{array}$ & 65 & Carboxylic acid metabolic process \\
\hline Dermcidin & 65 & Proteolytic and antimicrobial \\
\hline Ran-binding protein 9 & 61 & $\begin{array}{l}\text { Can act as an adapter protein to } \\
\text { couple membrane receptors to } \\
\text { intracellular signaling pathways, } \\
\text { also involved in microtubule } \\
\text { nucleation and axonal guidance. }\end{array}$ \\
\hline $\begin{array}{l}\text { Coiled-coil domain- } \\
\text { containing protein } 8\end{array}$ & 60 & $\begin{array}{l}\text { Negative regulation of } \\
\text { phosphatase activity, implicated in } \\
\text { dwarfism }\end{array}$ \\
\hline
\end{tabular}


Table 4-3. Mass-spectrometric analysis of immunopurified iNOS (Band 3)

\begin{tabular}{|c|c|c|}
\hline Protein & Mowse score & Function \\
\hline $\begin{array}{l}\text { Calcium-binding } \\
\text { mitochondrial carrier } \\
\text { protein }\end{array}$ & 330 & $\begin{array}{l}\text { Malate-Aspartate shuttle, } \\
\text { gluconeogenesis }\end{array}$ \\
\hline $\begin{array}{l}\text { Stress-induced- } \\
\text { phosphoprotein } 1\end{array}$ & 236 & $\begin{array}{l}\text { Assembly of molecular chaperones } \\
\text { HSC } 70 \text { and HSP90 complex }\end{array}$ \\
\hline $\begin{array}{l}\text { Insulin-like growth factor } \\
2 \text { mRNA-binding protein } \\
3\end{array}$ & 159 & $\begin{array}{l}\text { Regulation of cytokine } \\
\text { biosynthesis }\end{array}$ \\
\hline $\begin{array}{l}\text { Stress- } 70 \text { protein, } \\
\text { mitochondrial }\end{array}$ & 154 & $\begin{array}{l}\text { Chaperone; negatively regulates } \\
\text { apoptosis }\end{array}$ \\
\hline $\begin{array}{l}\text { Dolichyl- } \\
\text { diphosphooligosaccharide } \\
\text { glycosyltransferase } \\
\text { subunit } 1\end{array}$ & 76 & Glycosyltransferase activity \\
\hline
\end{tabular}


Table 4-4. Mass-spectrometric analysis of immunopurified iNOS (Band 4)

\begin{tabular}{|c|c|c|}
\hline Protein & Mowse score & Function \\
\hline $\begin{array}{l}\text { E3 ubiquitin-protein } \\
\text { ligase }\end{array}$ & 787 & $\begin{array}{l}\text { Ubiquitination and negatively } \\
\text { regulates mTOR pathway }\end{array}$ \\
\hline $\begin{array}{l}\text { Serine/threonine-protein } \\
\text { kinase } 38\end{array}$ & 321 & $\begin{array}{l}\text { Negative regulator of MAP } 3 \mathrm{~K} 1 / 2 \\
\text { signaling involved in regulation of } \\
\text { NF-kappaB signaling }\end{array}$ \\
\hline $\begin{array}{l}\text { Serine/threonine-protein } \\
\text { phosphatase } 2 \mathrm{~A} 55 \mathrm{kDa} \\
\text { regulatory subunit B } \\
\text { alpha isoform }\end{array}$ & 196 & $\begin{array}{l}\text { Binds oncogene TP53 which } \\
\text { contributes to transformation and } \\
\text { malignancy }\end{array}$ \\
\hline $\begin{array}{l}\text { Serine } \\
\text { palmitoyltransferase } 1\end{array}$ & 181 & $\begin{array}{l}\text { Sphingolipid metabolism and } \\
\text { homeostasis }\end{array}$ \\
\hline $\begin{array}{l}\text { Glutamate dehydrogenase } \\
1 \text { (GLUD1) }\end{array}$ & 109 & $\begin{array}{l}\text { Oxidation of Glutamate; Defects in } \\
\text { GLUD1 are the cause of familial } \\
\text { hyperinsulinemic hypoglycemia } \\
\text { type } 6\end{array}$ \\
\hline $\begin{array}{l}\text { MAP kinase-activated } \\
\text { protein kinase } 5\end{array}$ & 90 & $\begin{array}{l}\text { Tumor suppressor } \\
\text { serine/threonine-protein kinase } \\
\text { participating in mTOR signaling }\end{array}$ \\
\hline Nucleophosmin & 83 & $\begin{array}{l}\text { Proto-oncogene and wide-substrate } \\
\text { chaperone }\end{array}$ \\
\hline Striatin-4 & 66 & $\begin{array}{l}\text { Scaffolding protein for } \\
\text { calmodulin. Also binds } \\
\text { phosphatase } 2 \mathrm{~A}\end{array}$ \\
\hline $\begin{array}{l}\text { Nicotinamide } \\
\text { phosphoribosyltransferase }\end{array}$ & 64 & NAD biosynthesis pathway \\
\hline $\begin{array}{l}\text { Vacuolar protein sorting- } \\
\text { associated protein } 4 \mathrm{~A}\end{array}$ & 61 & $\begin{array}{l}\text { Membrane fission for endosomal } \\
\text { sorting }\end{array}$ \\
\hline
\end{tabular}


Table 4-5. Mass-spectrometric analysis of immunopurified iNOS (Band 5)

\begin{tabular}{|c|c|c|}
\hline Protein & Mowse score & Function \\
\hline Calmodulin & 797 & $\begin{array}{l}\text { Calmodulin- } \mathrm{Ca}^{2+} \text { control activity of } \\
\text { a large number of enzymes } \\
\text { especially kinases and } \\
\text { phosphatases, ion channels and } \\
\text { other proteins }\end{array}$ \\
\hline Calmodulin-like protein 3 & 143 & $\begin{array}{l}\text { Similar to calmodulin; gets } \\
\text { induced by TGF- } \beta 1\end{array}$ \\
\hline Selenoprotein $\mathrm{H}$ & 77 & Cell redox homeostasis \\
\hline
\end{tabular}


probability of matching $\mathrm{N}$ peaks by random chance. A protein sequence will be assigned a high Mowse score, with the high frequency of matched peaks or when a peak is exclusive.

The majority of the proteins immune-precipitated with iNOS were involved in protein sorting, processing and folding. Several proteins, including $78 \mathrm{kDa}$ glucoseregulated protein, are known to be the components of unfolded protein response (UPR) implying that the pathway gets activated in an iNOS- dwelling system or generally in an inflammatory profile. Also, several proteins were found to be involved in various pathological conditions and few could link iNOS and IBD manifestations. For example, catenin beta-1 has been linked to the development of colorectal cancer and another protein E3 UFM1-protein ligase acts as a tumor suppressor and negatively regulates NFkappaB transcription factor activity, which acts canonical to iNOS-upregulation, PP2A subunit B isoform B55-alpha can regulate dephosphorylation of the oncogene P53, MAPKAP kinase 5 is a tumor suppressor gene of mTOR pathway and nucleophosmin is proto-oncogenic. This is important, as it is already known that IBD patients are at a higher risk of developing colorectal cancer.

Several other proteins e.g., GLUD1, $78 \mathrm{kDa}$ glucose-regulated protein, were related to glucose metabolism or sensing. A recent report mentions the association of iNOS with low-order inflammatory states such as obesity-linked Type-2 diabetes (144). A major proportion of the protein was directly related but not limited to iNOS-pathway which include endoplasmin, serine/threonine-protein kinase TBK1, sulfhydryl oxidase 2, dermcidin, interleukin enhancer-binding factor 3, serine/threonine-protein kinase 38, insulin-like growth factor $2 \mathrm{mRNA}$-binding protein 3 to name a few.

Immunoprecipitation of calmodulin was interesting as iNOS is $\mathrm{Ca}^{2+}$ insensitive and calmodulin-regulation independent. Although, it is known that iNOS binds to calmodulin, with the abundance of iNOS within an inflammatory system it is possible that iNOS acts as calmodulin scavenger and modulate several important calmodulinregulated pathways. These results might be valuable in designing studies that center iNOS-dependent pathways in inflammatory disorders and explain many phenotypes associated with these diseases. 


\section{CHAPTER 5. DISCUSSION}

Pathophysiology of IBD-associated diarrhea is not clearly understood, and new molecular targets for its amelioration need to be identified. Spatial restriction of multiple proteins in the form of a macromolecular complex enables a tight and fine-tuned regulation of protein function. The goal of this study was to uncover a macromolecular interaction that is constituted and contributes to diarrhea in IBD and that may provide opportunities for possible therapeutic interventions.

\section{Pathological Roles of iNOS}

NO is an important cell signaling molecule which acts as a mediator of several physiological functions such as e.g. neurotransmission, smooth muscle relaxation, wound repair and immunomodulation (68). However, the physiological benefits are limited to NO being synthesized when required for a brief period lasting up to few minutes (145). When there is chronic NO production, it becomes pathophysiological. There is a prevailing notion that iNOS upregulation is a cytoprotective response to an inflammatory challenge. However, as NO production by iNOS is sustained it becomes cytotoxic. iNOS function is now being implicated in the pathogenesis of a number of neurodegenerative disorders such as amyotrophic lateral sclerosis, Alzheimers and Parkinson diseases, cardiovascular diseases such as atherosclerosis and lung disorders such as chronic bronchitis, asthma and emphysema, and metabolic disorders such as obesity-linked insulin resistance. Many aspects for a role of iNOS in maintenance of an inflammatory profile have been shown. Also, pharmacological manipulations to inhibit iNOS function and knockout of iNOS in experimental colitis models and emphysema models have been shown to lead to resolution of inflammation $(112,141)$. Noticeably, the temporal resolution of NO-mediated effects was critical to achieve consistency in these studies. However, there have been conflicting reports on the role of $\mathrm{NO}$ in water and electrolyte secretion in colitis (112).

\section{Compartmentalized NO-cGMP Signaling}

Very few studies have reported compartmentation of NO-cGMP signaling. We demonstrated that iNOS forms clusters near the plasma membrane in UC-affected gut epithelial cells. Dimerization of iNOS is a prerequisite for its function. It is quite possible that the clusters are comprised of iNOS multimers representative of a functional state of iNOS in UC-affected tissue. Previous data show that NOS activities can be regulated through protein-protein interactions and lipid modifications by governing their specific intracellular locations and also the downstream events will be dependent on the amounts of NO synthesized (33). For example, sGC gets stimulated with the nanomolar amounts of NO, while peroxynitrites start getting synthesized in the micromolar range (33). All these factors will critically control the spatiotemporal regulation of the NO-cGMP

pathway. We demonstrated a high-affinity interaction of iNOS with the PDZ-scaffolding 
protein NHERF2. A consistent observation with PDZ proteins is their predominant association with plasma membrane and their unique binding specificities contributing to formation of distinct complexes (43). This organizational capacity of PDZ proteins has also been instrumental in recruiting many cytosolic proteins to the membrane and mediating vectorial functions $(69,70)$. It is highly unlikely that large multiprotein signaling complexes assemble randomly. The protein expression and localization is spatiotemporally coordinated, further aided by specific post-translational modifications (146). Palmitoylation of NOS2 on Cys3 as well as interaction with the PDZ-proteins CAP-70 or EBP50 is essential to mediate its apical targeting $(69,70,147)$. Also, these interactions resulted in increased NOS2 dimerization essential for enzyme function (69). It is very likely that iNOS-NHERF2 interaction will determine the plasma membrane localization of iNOS in IBD where it can be involved in vectorial physiology. The pathophysiological relevance of iNOS-NHERF2 interaction at the apical plasma membrane was demonstrated using PLA. This is a first demonstration of protein-protein interaction in a human disease tissue where the sample comprises of the UC-affected and unaffected areas of the same patient. It is quite possible if the interaction between iNOS and NHERF2 is compromised the iNOS potency to exacerbate disease pathology would decrease.

It is conceivable from the fact of CFTR expression levels being unaltered in UC as demonstrated with heat analysis of mRNA expression in human biopsies and immunohistochemistry in UC-affected biopsies, that CFTR hyperactivation as hypothesized in UC is more a regulatory phenomenon than mere increase in the protein expression level. We demonstrate that CFTR regulation in IBD happens in the form of CFTR being functionally coupled to iNOS in restricted microdomains at the plasma membrane. It also needs to be emphasized that proximity of a cGMP-generating system (iNOS and GC) needs to be proximal to the cGMP-effector system (PKG and CFTR) for a compartmentalized regulation which is demonstrated in the current study to be achieved in the form of a macromolecular signaling complex.

\section{Diarrhea in Inflammatory Bowel Disorders}

Many surface proteins such as bacterial receptors and transporters, and scaffolding proteins have been identified to increase IBD susceptibility. A list of the selective IBD susceptibility genes is shown in Table 5-1. Identification of these susceptibility factors partially explain the molecular phenotype of IBD and has immensely contributed in dissecting out the mechanisms that lead to IBD. However, there is a huge lack of information about the interplay of various factors that cause IBD which makes it still challenging to be managed.

Diarrhea in IBD presents a big obstacle for its effective management. Diarrhea is a consequence of dysfunctional homeostasis when pro-absorptive and anti-secretory mechanisms fail to be compensated. Net $\mathrm{Na}^{+}$absoprtion and $\mathrm{Cl}^{-}$secretion into the lumen set the osmotic gradients for hydro flux. Thus, it is critical to understand how $\mathrm{Na}^{+}$ absorption and active $\mathrm{Cl}^{-}$secretion may get disturbed and imbalanced in IBD and cause 
Table 5-1. IBD susceptibility genes

\begin{tabular}{|c|c|c|}
\hline Gene & Implications & References \\
\hline NOD2/CARD 15 & $\begin{array}{l}\text { Intracellular bacterial } \\
\text { receptor }\end{array}$ & $(148,149)$ \\
\hline NODI/CARD 4 & $\begin{array}{l}\text { Intracellular bacterial } \\
\text { receptor }\end{array}$ & $(150)$ \\
\hline $\begin{array}{l}\text { IL23R, TNFSF 15, } \\
\text { STAT3, IL12B, CCR6 } \\
\text { and JAK2 }\end{array}$ & Interleukin and related genes & $(151)$ \\
\hline OCTN1/N2 & Organic Cation Transporters & $(152)$ \\
\hline MDRl & ABC-transporter & $(153)$ \\
\hline CFTR & ABC-transporter & $(105)$ \\
\hline$D L G 5$ & PDZ protein & $(154)$ \\
\hline$G P X 1, G P X 2$ & Peroxidases & $(151)$ \\
\hline ATG16L1, IRGM & Autophagy & $(155)$ \\
\hline
\end{tabular}


diarrhea. Net $\mathrm{Na}^{+}, \mathrm{Cl}^{-}$and water absorption has been found to be reduced in UC patients and in patients with active $\mathrm{CD}$. Decreased $\mathrm{Na}^{+}$absorption is deduced to be majorly a consequence of down-regulated expression of $\mathrm{Na}^{+}$transporters and their regulatory proteins which include NHE1 and 3 (excluding NHE2), aldosterone responsive $\beta$ and $\gamma$ subunits of ENaC, $\mathrm{Na}^{+} / \mathrm{K}^{+}$-ATPase- $\alpha$, and NHERF proteins $(156,157)$. Also, NHE1 \& $3, \beta-\mathrm{ENaC}$, and NHERF1 \& 2 were found to be similarly down-regulated in experimental colitis models (157). Additionally, NO could dose-dependently inhibit NHE3 activity in Caco-2 cells (158). NHE3 couples strongly with NHERF proteins that inhibit the channel function through cellular messengers-cAMP, cGMP and calcium and NHERF2 has been found to regulate plasma membrane levels of NHE3 $(159,160)$. However, the involvement of NHE3-mediated $\mathrm{Na}^{+}$absorption and its dysregulation in NO-pooled IBDaffected mucosa would still be debatable with the precarious consideration of the fact that NHE3 is poorly expressed in the colonic mucosa (161) and therefore, will have minimal contribution towards $\mathrm{UC}$-associated diarrhea. Speaking of $\mathrm{Cl}^{-}$secretory mechanisms in IBD, the $\mathrm{Cl}^{-}-\mathrm{HCO}_{3}{ }^{-}$exchanger down-regulated in-adenoma (DRA) present on the apical membrane has reduced mRNA and protein levels in UC (156). However, very little data exist on supporting the contribution of active anion secretion in IBD-associated diarrhea. Also, the proinflammatory cytokines which also have been shown to play important role in diarrhea failed to consistently affect anion secretion $(103,156)$. However, it needs to be understood that IBD is a complicated disorder with innumerable pathological events taking place concurrently. Also, the process of fluid secretion across the mucosal epithelia is highly synergistic. Therefore, it is hard to functionally dissect out individual contributions to electrolyte abnormalities in a whole ensemble of transporters in the affected tissue. CFTR is a dominant player of the fluid secretory pathway of the gut to the extent that CF mice did not secrete fluid in response to cholera toxin and they suffer from meconium ileus (90). Therefore, how CFTR behaves functionally in IBD is critical to be elucidated. Alterations in the mRNA or protein levels of CFTR have not been conclusively determined. We demonstrated a mechanism in which CFTR gets regulated in an iNOS-governed cGMP microdomain which ultimately contributes to diarrhea in IBD. We demonstrated the concept in vitro by inducing iNOS levels in response to cytokine challenge (cytomix) in colon epithelial cells and stimulating CFTR $\mathrm{Cl}^{-}$channel function in the presence of a cGMP-elevating agent. Further the colocalization studies in polarized colon epithelial cells confirmed that proximity of CFTR to iNOS at the apical plasma membrane is the molecular basis of iNOS-dependent potentiation of CFTR Clchannel function.

\section{PDZ-scaffolds in the Spatiotemporal Regulation of CFTR}

NHERF2 has been demonstrated to be acting as a GKAP for cGKII for the cGMP-mediated regulation of NHE3 (30). Also, cGKII is known to be the isoform to mediate cGMP-responsive activation of CFTR. It is quite possible that NHERF2 exhibits dual role of assembling CFTR and iNOS at the plasma membrane as well as functioning as GKAP in the complex to spatiotemporally regulate CFTR function. NHE3 inhibition was specific to NHERF2-cGKII complex as NHERF1 could not compensate for the effect. The likely reason for NHERF1 to not to be able to constitute CFTR-iNOS 
complex in IBD, although, NHERF1 is a common binding partner for both the proteins, is because of its inability to function as GKAP and also may be due to the subtle differences in their locations or squences (30). Therefore, it is conceivable that deficiency of NHERF2 would impair CFTR-iNOS complex formation and ameliorate diarrhea in colitis. Using DSS-induced colitis, we demonstrated in vivo that Nherf $^{-/-}$mice are partially protected against diarrhea as indicated by reduced weight loss, intestinal fluid secretion, and wet/dry weight in the Nherf $2^{-/}$mice. Also, the overall pathological features of DSS colitis improved in Nherf $2^{-/}$mice as suggested by histological examination. This observation may pertain to the partial failure of iNOS to be properly localized inside the cell in the absence of NHERF2 so that the debilitating effects of iNOS function on mucosal integrity are mitigated. The biological significance of NHERF proteins in mediating highly compartmentalized regulation of CFTR was recently recapitulated in various PDZ-protein knockout models (49). Despite the fact that NHERF proteins exhibit high sequence homology, overlapping tissue expression and subcellular localizations and are predicted to be redundant based on physiological normalcy of selective knockouts, their deficiencies produced discrete phenotypes of CFTR function. Fsk-mediated stimulation of CFTR-dependent bicarbonate secretion was highly reduced in Nherfl ${ }^{-/}$ and $\beta_{2}$-AR agonist failed to stimulate CFTR function altogether (49). Nherf $2^{-/}$mice exhibited higher Fsk-stimulated CFTR bicarbonate secretion and the accounting mechanism was found to be LPA- mediated inhibition of CFTR function which requires NHERF2 scaffolding. Also, it was concluded that NHERF1 and NHERF2 can function independently of one another. Ablation of PDZK1 had no effect on basal and Fskstimulated CFTR secretions. PDZK1 couples CFTR and MRP4 in a macromolecular complex and MRP4 inhibition augments CFTR Cl- channel function. Based on this observation, $P d z k 1^{-/}$mice should have increased CFTR function. However, it is important to consider that MRP4 regulates CFTR function in a highly compartmentalized manner. Therefore, CFTR potentiation in response to Fsk which causes massive CFTR activation can easily mask compartmentalized activation of CFTR in the CFTR-PDZK1MRP4 complex and therefore, Fsk is not an ideal reagent to conclude for the loss of compartmentalized-downregulation of CFTR function in $P d z k 1^{--}$mice. Additionally, the studies were performed in the duodenal tissue which expresses very small amounts of MRP4.

\section{CFTR within the NO-governed Compartments}

We observe that if iNOS function is inhibited within the complex with the iNOSinhibitor (L-NIL) in DSS-induced colitis, diarrhea is attenuated. However, the effect observed was small which might be due to the nature of the mechanism by which iNOS activates CFTR, which is a highly compartmentalized signaling. Whether CFTR within the complex is subject to NO-mediated S-nitrosylation, a common NO-mediated posttranslational modification of the protein targets, and be regulated by the process is a matter of contemplation and further studies. The process of S-nitrosylation would involve nitration of tyrosine residues, which is usually a molecular consequence of high concentration of NO, a process that will accelerate CFTR degradation (162). However, this does not reconcile with the augmentation of CFTR function and unaltered protein 
expression in IBD-affected vs. normal mucosal tissue. It is quite possible that the amount of NO within the CFTR-iNOS micro-compartments is not sufficient to induce Snitrosylation as NO is quickly neutralized by sGC in close proximity. The physiological concentrations of S-nitrosoglutathione (GSNO), the endogenous NO-donor and bronchodilator, has been shown to increase expression, maturation, and function of both the wild-type and the $\triangle F 508$ CFTR (162). Whether S-nitrosylation of CFTR is involved in GSNO-mediated effects is still not established. However, the mechanisms have been broadly elucidated to be cGMP-dependent and independent (163). This aspect of iNOS mediated regulation of CFTR in IBD would be a matter for future investigation.

\section{Dissolution of Protein-protein Complexes as a Potential Therapy for IBD}

Given the fact that the molecular composition of CFTR-containing complexes has been shown to significantly influence the secretory profile of the gut and that it can also culminate in a pathological condition, modulating such macromolecular associations can be a valuable therapeutic approach. Also, with the restriction of mechanism within the complex, this approach can be highly specific. Administration of a cell permeable $\mathrm{LPA}_{2}$ specific peptide could disrupt the CFTR-NHERF2-LPA 2 structural and consequently, functional integrity. Also, inclusion of a competing peptide for MRP4-CFTR interaction attenuated MK571 (an MRP4 inhibitor) - induced potentiation of CFTR function (5). Our lab recently identified a cell-permeable, small-molecule compound, CO-068, that preferentially inhibits LPA $2-$ NHERF2 interaction and augments CFTR activity in Calu-3 cells and CFTR-dependent fluid secretion from pig tracheal submucosal glands (164). Also, recent studies in our lab and by others have demonstrated that the functional rescue of $\triangle \mathrm{F} 508$-CFTR depends upon the macromolecular associations of the mutated protein and it is quite possible that several drugs known to correct mutant protein functional deficiencies would involve modulation of protein-protein interactions of CFTR (165). Recently, a NHERF1-mediated complex of chemokine receptor CXCR2 with PLC- $\beta_{2}$ has been shown to regulate neutrophil infiltration during inflammation (166). Also, addition of CXCR2-specific peptide (containing the PDZ motif) was found to suppress neutrophilic chemotaxis and transepithelial migration of potential therapeutic relevance to inflammatory disorders such as colitis, chronic lung inflammation and atherosclerosis (166). Therefore, in our opinion specific disruption of iNOS-NHERF2 interaction will likely attenuate UC-associated diarrhea and improve one of the most common symptoms of UC.

Although it is tempting to speculate that regulation of CFTR function through iNOS-elicited cGMP signaling will be a general mechanism in other inflammatory phenotypes such as lung inflammation and pancreatitis wherein all these proteins are present, we think extra caution should be exercised for such generalization since these phenotypes largely depend upon the organ type. However, given the many similarities shared by UC and related inflammatory disorders of the gut such as Crohn's disease and diverticulitis, our findings will help understand the associated diarrheal phenotype. In short, our findings will advance the understanding of mechanisms accounting for the diarrheal manifestations in IBD and help identify new targets and approaches to control 
the disease. Our study also has important implications for targeting other forms of gastrointestinal inflammation. 


\section{LIST OF REFERENCES}

1. Rehmann, H., Wittinghofer, A., and Bos, J.L. 2007. Capturing cyclic nucleotides in action: snapshots from crystallographic studies. Nat Rev Mol Cell Biol 8:63-73.

2. Cheepala, S., Hulot, J.S., Morgan, J.A., Sassi, Y., Zhang, W., Naren, A.P., and Schuetz, J.D. 2013. Cyclic Nucleotide Compartmentalization: Contributions of Phosphodiesterases and ATP-Binding Cassette Transporters. Annu Rev Pharmacol Toxicol 53:231-253.

3. Baillie, G.S., Scott, J.D., and Houslay, M.D. 2005. Compartmentalisation of phosphodiesterases and protein kinase A: opposites attract. FEBS Lett 579:32643270 .

4. Penmatsa, H., Zhang, W., Yarlagadda, S., Li, C., Conoley, V.G., Yue, J., Bahouth, S.W., Buddington, R.K., Zhang, G., Nelson, D.J., et al. 2010. Compartmentalized cyclic adenosine 3',5'-monophosphate at the plasma membrane clusters PDE3A and cystic fibrosis transmembrane conductance regulator into microdomains. Mol Biol Cell 21:1097-1110.

5. Li, C., Krishnamurthy, P.C., Penmatsa, H., Marrs, K.L., Wang, X.Q., Zaccolo, M., Jalink, K., Li, M., Nelson, D.J., Schuetz, J.D., et al. 2007. Spatiotemporal coupling of cAMP transporter to CFTR chloride channel function in the gut epithelia. Cell 131:940-951.

6. Cobb, B.R., Fan, L., Kovacs, T.E., Sorscher, E.J., and Clancy, J.P. 2003. Adenosine receptors and phosphodiesterase inhibitors stimulate $\mathrm{Cl}$ - secretion in Calu-3 cells. Am J Respir Cell Mol Biol 29:410-418.

7. Cooper, D.M., and Crossthwaite, A.J. 2006. Higher-order organization and regulation of adenylyl cyclases. Trends Pharmacol Sci 27:426-431.

8. Zippin, J.H., Farrell, J., Huron, D., Kamenetsky, M., Hess, K.C., Fischman, D.A., Levin, L.R., and Buck, J. 2004. Bicarbonate-responsive "soluble" adenylyl cyclase defines a nuclear cAMP microdomain. J Cell Biol 164:527-534.

9. Ostrom, R.S., and Insel, P.A. 2004. The evolving role of lipid rafts and caveolae in $\mathrm{G}$ protein-coupled receptor signaling: implications for molecular pharmacology. Br J Pharmacol 143:235-245.

10. Wong, W., and Scott, J.D. 2004. AKAP signalling complexes: focal points in space and time. Nat Rev Mol Cell Biol 5:959-970. 
11. Nikolaev, V.O., Gambaryan, S., Engelhardt, S., Walter, U., and Lohse, M.J. 2005. Real-time monitoring of the PDE2 activity of live cells: hormone-stimulated cAMP hydrolysis is faster than hormone-stimulated cAMP synthesis. $J$ Biol Chem 280:1716-1719.

12. Zaccolo, M. 2006. Phosphodiesterases and compartmentalized cAMP signalling in the heart. Eur J Cell Biol 85:693-697.

13. Lissandron, V., Terrin, A., Collini, M., D'Alfonso, L., Chirico, G., Pantano, S., and Zaccolo, M. 2005. Improvement of a FRET-based indicator for cAMP by linker design and stabilization of donor-acceptor interaction. J Mol Biol 354:546555.

14. Zaccolo, M., and Pozzan, T. 2002. Discrete microdomains with high concentration of cAMP in stimulated rat neonatal cardiac myocytes. Science 295:1711-1715.

15. Dodge, K.L., Khouangsathiene, S., Kapiloff, M.S., Mouton, R., Hill, E.V., Houslay, M.D., Langeberg, L.K., and Scott, J.D. 2001. mAKAP assembles a protein kinase $\mathrm{A} / \mathrm{PDE} 4$ phosphodiesterase cAMP signaling module. $E M B O J$ 20:1921-1930.

16. Coghlan, V.M., Hausken, Z.E., and Scott, J.D. 1995. Subcellular targeting of kinases and phosphatases by association with bifunctional anchoring proteins. Biochem Soc Trans 23:592-596.

17. Klauck, T.M., Faux, M.C., Labudda, K., Langeberg, L.K., Jaken, S., and Scott, J.D. 1996. Coordination of three signaling enzymes by AKAP79, a mammalian scaffold protein. Science 271:1589-1592.

18. Dessauer, C.W. 2009. Adenylyl cyclase--A-kinase anchoring protein complexes: the next dimension in cAMP signaling. Mol Pharmacol 76:935-941.

19. Dransfield, D.T., Bradford, A.J., Smith, J., Martin, M., Roy, C., Mangeat, P.H., and Goldenring, J.R. 1997. Ezrin is a cyclic AMP-dependent protein kinase anchoring protein. EMBOJ 16:35-43.

20. Bretscher, A., Edwards, K., and Fehon, R.G. 2002. ERM proteins and merlin: integrators at the cell cortex. Nat Rev Mol Cell Biol 3:586-599.

21. Weinman, E.J., Wang, Y., Wang, F., Greer, C., Steplock, D., and Shenolikar, S. 2003. A C-terminal PDZ motif in NHE3 binds NHERF-1 and enhances cAMP inhibition of sodium-hydrogen exchange. Biochemistry 42:12662-12668. 
22. Naren, A.P., Cobb, B., Li, C., Roy, K., Nelson, D., Heda, G.D., Liao, J., Kirk, K.L., Sorscher, E.J., Hanrahan, J., et al. 2003. A macromolecular complex of beta 2 adrenergic receptor, CFTR, and ezrin/radixin/moesin-binding phosphoprotein 50 is regulated by PKA. Proc Natl Acad Sci U S A 100:342-346.

23. Haggie, P.M., Stanton, B.A., and Verkman, A.S. 2004. Increased diffusional mobility of CFTR at the plasma membrane after deletion of its C-terminal PDZ binding motif. J Biol Chem 279:5494-5500.

24. Schuetz, J.D., Connelly, M.C., Sun, D., Paibir, S.G., Flynn, P.M., Srinivas, R.V., Kumar, A., and Fridland, A. 1999. MRP4: A previously unidentified factor in resistance to nucleoside-based antiviral drugs. Nat Med 5:1048-1051.

25. Chen, Z.S., Lee, K., and Kruh, G.D. 2001. Transport of cyclic nucleotides and estradiol 17-beta-D-glucuronide by multidrug resistance protein 4 . Resistance to 6-mercaptopurine and 6-thioguanine. J Biol Chem 276:33747-33754.

26. Sassi, Y., Lipskaia, L., Vandecasteele, G., Nikolaev, V.O., Hatem, S.N., Cohen Aubart, F., Russel, F.G., Mougenot, N., Vrignaud, C., Lechat, P., et al. 2008. Multidrug resistance-associated protein 4 regulates cAMP-dependent signaling pathways and controls human and rat SMC proliferation. J Clin Invest 118:27472757.

27. Sinha, C., Ren, A., Arora, K., Moon, C.S., Yarlagadda, S., Zhang, W., Cheepala, S.B., Schuetz, J.D., and Naren, A.P. 2013. Multi-drug resistance protein 4 (MRP4)-mediated regulation of fibroblast cell migration reflects a dichotomous role of intracellular cyclic nucleotides. J Biol Chem 288:3786-3794.

28. Lucas, K.A., Pitari, G.M., Kazerounian, S., Ruiz-Stewart, I., Park, J., Schulz, S., Chepenik, K.P., and Waldman, S.A. 2000. Guanylyl cyclases and signaling by cyclic GMP. Pharmacol Rev 52:375-414.

29. Guo, D., Tan, Y.C., Wang, D., Madhusoodanan, K.S., Zheng, Y., Maack, T., Zhang, J.J., and Huang, X.Y. 2007. A Rac-cGMP signaling pathway. Cell 128:341-355.

30. Cha, B., Kim, J.H., Hut, H., Hogema, B.M., Nadarja, J., Zizak, M., Cavet, M., Lee-Kwon, W., Lohmann, S.M., Smolenski, A., et al. 2005. cGMP inhibition of $\mathrm{Na}+/ \mathrm{H}+$ antiporter 3 (NHE3) requires PDZ domain adapter NHERF2, a broad specificity protein kinase G-anchoring protein. $J$ Biol Chem 280:16642-16650.

31. Reierson, G.W., Guo, S., Mastronardi, C., Licinio, J., and Wong, M.L. 2011. cGMP Signaling, Phosphodiesterases and Major Depressive Disorder. Curr Neuropharmacol 9:715-727. 
32. Zaccolo, M., and Movsesian, M.A. 2007. cAMP and cGMP signaling cross-talk: role of phosphodiesterases and implications for cardiac pathophysiology. Circ Res 100:1569-1578.

33. Madhusoodanan, K.S., and Murad, F. 2007. NO-cGMP signaling and regenerative medicine involving stem cells. Neurochem Res 32:681-694.

34. Li, C., and Naren, A.P. 2010. CFTR chloride channel in the apical compartments: spatiotemporal coupling to its interacting partners. Integr Biol (Camb) 2:161-177.

35. Rowe, S.M., Miller, S., and Sorscher, E.J. 2005. Cystic fibrosis. N Engl J Med 352:1992-2001.

36. Naren, A.P., Cormet-Boyaka, E., Fu, J., Villain, M., Blalock, J.E., Quick, M.W., and Kirk, K.L. 1999. CFTR chloride channel regulation by an interdomain interaction. Science 286:544-548.

37. Li, C., and Naren, A.P. 2005. Macromolecular complexes of cystic fibrosis transmembrane conductance regulator and its interacting partners. Pharmacol Ther 108:208-223.

38. Poulsen, J.H., Fischer, H., Illek, B., and Machen, T.E. 1994. Bicarbonate conductance and $\mathrm{pH}$ regulatory capability of cystic fibrosis transmembrane conductance regulator. Proc Natl Acad Sci U S A 91:5340-5344.

39. Welsh, M.J., and Smith, A.E. 1993. Molecular mechanisms of CFTR chloride channel dysfunction in cystic fibrosis. Cell 73:1251-1254.

40. Naren, A.P., Nelson, D.J., Xie, W., Jovov, B., Pevsner, J., Bennett, M.K., Benos, D.J., Quick, M.W., and Kirk, K.L. 1997. Regulation of CFTR chloride channels by syntaxin and Munc18 isoforms. Nature 390:302-305.

41. Huang, P., Lazarowski, E.R., Tarran, R., Milgram, S.L., Boucher, R.C., and Stutts, M.J. 2001. Compartmentalized autocrine signaling to cystic fibrosis transmembrane conductance regulator at the apical membrane of airway epithelial cells. Proc Natl Acad Sci U S A 98:14120-14125.

42. $\quad$ Li, C., Dandridge, K.S., Di, A., Marrs, K.L., Harris, E.L., Roy, K., Jackson, J.S., Makarova, N.V., Fujiwara, Y., Farrar, P.L., et al. 2005. Lysophosphatidic acid inhibits cholera toxin-induced secretory diarrhea through CFTR-dependent protein interactions. $J$ Exp Med 202:975-986.

43. Hung, A.Y., and Sheng, M. 2002. PDZ domains: structural modules for protein complex assembly. J Biol Chem 277:5699-5702. 
44. Lee, H.J., and Zheng, J.J. 2010. PDZ domains and their binding partners: structure, specificity, and modification. Cell Commun Signal 8:8.

45. Zhang, M., and Wang, W. 2003. Organization of signaling complexes by PDZdomain scaffold proteins. Acc Chem Res 36:530-538.

46. Dev, K.K. 2004. Making protein interactions druggable: targeting PDZ domains. Nat Rev Drug Discov 3:1047-1056.

47. Guggino, W.B., and Stanton, B.A. 2006. New insights into cystic fibrosis: molecular switches that regulate CFTR. Nat Rev Mol Cell Biol 7:426-436.

48. Raghuram, V., Mak, D.O., and Foskett, J.K. 2001. Regulation of cystic fibrosis transmembrane conductance regulator single-channel gating by bivalent PDZdomain-mediated interaction. Proc Natl Acad Sci U S A 98:1300-1305.

49. Singh, A.K., Riederer, B., Krabbenhoft, A., Rausch, B., Bonhagen, J., Lehmann, U., de Jonge, H.R., Donowitz, M., Yun, C., Weinman, E.J., et al. 2009. Differential roles of NHERF1, NHERF2, and PDZK1 in regulating CFTRmediated intestinal anion secretion in mice. J Clin Invest 119:540-550.

50. Li, C., Roy, K., Dandridge, K., and Naren, A.P. 2004. Molecular assembly of cystic fibrosis transmembrane conductance regulator in plasma membrane. J Biol Chem 279:24673-24684.

51. Luo, X., Choi, J.Y., Ko, S.B., Pushkin, A., Kurtz, I., Ahn, W., Lee, M.G., and Muallem, S. 2001. HCO3- salvage mechanisms in the submandibular gland acinar and duct cells. J Biol Chem 276:9808-9816.

52. Yun, C.H., Oh, S., Zizak, M., Steplock, D., Tsao, S., Tse, C.M., Weinman, E.J., and Donowitz, M. 1997. cAMP-mediated inhibition of the epithelial brush border $\mathrm{Na}+/ \mathrm{H}+$ exchanger, NHE3, requires an associated regulatory protein. Proc Natl Acad Sci U S A 94:3010-3015.

53. Choi, J.Y., Muallem, D., Kiselyov, K., Lee, M.G., Thomas, P.J., and Muallem, S. 2001. Aberrant CFTR-dependent HCO3- transport in mutations associated with cystic fibrosis. Nature 410:94-97.

54. Haggie, P.M., Kim, J.K., Lukacs, G.L., and Verkman, A.S. 2006. Tracking of quantum dot-labeled CFTR shows near immobilization by C-terminal PDZ interactions. Mol Biol Cell 17:4937-4945. 
55. Lamprecht, G., Heil, A., Baisch, S., Lin-Wu, E., Yun, C.C., Kalbacher, H., Gregor, M., and Seidler, U. 2002. The down regulated in adenoma (dra) gene product binds to the second PDZ domain of the NHE3 kinase A regulatory protein (E3KARP), potentially linking intestinal $\mathrm{Cl}-/ \mathrm{HCO} 3-$ exchange to $\mathrm{Na}+/ \mathrm{H}+$ exchange. Biochemistry 41:12336-12342.

56. Mount, D.B., and Romero, M.F. 2004. The SLC26 gene family of multifunctional anion exchangers. Pflugers Arch 447:710-721.

57. Ko, S.B., Zeng, W., Dorwart, M.R., Luo, X., Kim, K.H., Millen, L., Goto, H., Naruse, S., Soyombo, A., Thomas, P.J., et al. 2004. Gating of CFTR by the STAS domain of SLC26 transporters. Nat Cell Biol 6:343-350.

58. Gray, M.A. 2004. Bicarbonate secretion: it takes two to tango. Nat Cell Biol 6:292-294.

59. Lu, M., Leng, Q., Egan, M.E., Caplan, M.J., Boulpaep, E.L., Giebisch, G.H., and Hebert, S.C. 2006. CFTR is required for PKA-regulated ATP sensitivity of Kir1.1 potassium channels in mouse kidney. J Clin Invest 116:797-807.

60. Chan, H.C., Shi, Q.X., Zhou, C.X., Wang, X.F., Xu, W.M., Chen, W.Y., Chen, A.J., Ni, Y., and Yuan, Y.Y. 2006. Critical role of CFTR in uterine bicarbonate secretion and the fertilizing capacity of sperm. Mol Cell Endocrinol 250:106-113.

61. Pietrement, C., Da Silva, N., Silberstein, C., James, M., Marsolais, M., Van Hoek, A., Brown, D., Pastor-Soler, N., Ameen, N., Laprade, R., et al. 2008. Role of NHERF1, cystic fibrosis transmembrane conductance regulator, and cAMP in the regulation of aquaporin 9. J Biol Chem 283:2986-2996.

62. Rojek, A.M., Skowronski, M.T., Fuchtbauer, E.M., Fuchtbauer, A.C., Fenton, R.A., Agre, P., Frokiaer, J., and Nielsen, S. 2007. Defective glycerol metabolism in aquaporin 9 (AQP9) knockout mice. Proc Natl Acad Sci U S A 104:3609-3614.

63. King, L.S., Kozono, D., and Agre, P. 2004. From structure to disease: the evolving tale of aquaporin biology. Nat Rev Mol Cell Biol 5:687-698.

64. Cheung, K.H., Leung, C.T., Leung, G.P., and Wong, P.Y. 2003. Synergistic effects of cystic fibrosis transmembrane conductance regulator and aquaporin-9 in the rat epididymis. Biol Reprod 68:1505-1510.

65. Yoo, D., Flagg, T.P., Olsen, O., Raghuram, V., Foskett, J.K., and Welling, P.A. 2004. Assembly and trafficking of a multiprotein ROMK (Kir 1.1) channel complex by PDZ interactions. J Biol Chem 279:6863-6873. 
66. Grisham, M.B., Jourd'Heuil, D., and Wink, D.A. 1999. Nitric oxide. I. Physiological chemistry of nitric oxide and its metabolites:implications in inflammation. Am J Physiol 276:G315-321.

67. Zhang, W., Kuncewicz, T., Yu, Z.Y., Zou, L., Xu, X., and Kone, B.C. 2003. Protein-protein interactions involving inducible nitric oxide synthase. Acta Physiol Scand 179:137-142.

68. Aktan, F. 2004. iNOS-mediated nitric oxide production and its regulation. Life Sci 75:639-653.

69. Navarro-Lerida, I., Martinez-Moreno, M., Ventoso, I., Alvarez-Barrientos, A., and Rodriguez-Crespo, I. 2007. Binding of CAP70 to inducible nitric oxide synthase and implications for the vectorial release of nitric oxide in polarized cells. Mol Biol Cell 18:2768-2777.

70. Glynne, P.A., Darling, K.E., Picot, J., and Evans, T.J. 2002. Epithelial inducible nitric-oxide synthase is an apical EBP50-binding protein that directs vectorial nitric oxide output. J Biol Chem 277:33132-33138.

71. Villanueva, C., and Giulivi, C. 2010. Subcellular and cellular locations of nitric oxide synthase isoforms as determinants of health and disease. Free Radic Biol Med 49:307-316.

72. Iwakiri, Y., Satoh, A., Chatterjee, S., Toomre, D.K., Chalouni, C.M., Fulton, D., Groszmann, R.J., Shah, V.H., and Sessa, W.C. 2006. Nitric oxide synthase generates nitric oxide locally to regulate compartmentalized protein S-

nitrosylation and protein trafficking. Proc Natl Acad Sci U S A 103:19777-19782.

73. Leeb-Lundberg, L.M., Marceau, F., Muller-Esterl, W., Pettibone, D.J., and Zuraw, B.L. 2005. International union of pharmacology. XLV. Classification of the kinin receptor family: from molecular mechanisms to pathophysiological consequences. Pharmacol Rev 57:27-77.

74. Wyckoff, M.H., Chambliss, K.L., Mineo, C., Yuhanna, I.S., Mendelsohn, M.E., Mumby, S.M., and Shaul, P.W. 2001. Plasma membrane estrogen receptors are coupled to endothelial nitric-oxide synthase through Galpha(i). J Biol Chem 276:27071-27076.

75. Kolios, G., Valatas, V., and Ward, S.G. 2004. Nitric oxide in inflammatory bowel disease: a universal messenger in an unsolved puzzle. Immunology 113:427-437.

76. Garthwaite, J. 2007. Neuronal nitric oxide synthase and the serotonin transporter get harmonious. Proc Natl Acad Sci US A 104:7739-7740. 
77. Barrett, K.E., and Keely, S.J. 2000. Chloride secretion by the intestinal epithelium: molecular basis and regulatory aspects. Annu Rev Physiol 62:535-572.

78. Vaandrager, A.B., Tilly, B.C., Smolenski, A., Schneider-Rasp, S., Bot, A.G., Edixhoven, M., Scholte, B.J., Jarchau, T., Walter, U., Lohmann, S.M., et al. 1997. cGMP stimulation of cystic fibrosis transmembrane conductance regulator $\mathrm{Cl}$ channels co-expressed with cGMP-dependent protein kinase type II but not type Ibeta. J Biol Chem 272:4195-4200.

79. Knowles, M., Gatzy, J., and Boucher, R. 1981. Increased bioelectric potential difference across respiratory epithelia in cystic fibrosis. N Engl J Med 305:14891495.

80. Knowles, M.R., Paradiso, A.M., and Boucher, R.C. 1995. In vivo nasal potential difference: techniques and protocols for assessing efficacy of gene transfer in cystic fibrosis. Hum Gene Ther 6:445-455.

81. Yarlagadda, S., Zhang, W., Penmatsa, H., Ren, A., Arora, K., Naren, A.P., Khan, F.A., Donnellan, C.A., Srinivasan, S., Stokes, D.C., et al. 2012. A young Hispanic with c.1646G $>$ A mutation exhibits severe cystic fibrosis lung disease: is ivacaftor an option for therapy? Am J Respir Crit Care Med 186:694-696.

82. Rowntree, R.K., and Harris, A. 2003. The phenotypic consequences of CFTR mutations. Ann Hum Genet 67:471-485.

83. Amaral, M.D. 2004. CFTR and chaperones: processing and degradation. $J$ Mol Neurosci 23:41-48.

84. Serohijos, A.W., Hegedus, T., Aleksandrov, A.A., He, L., Cui, L., Dokholyan, N.V., and Riordan, J.R. 2008. Phenylalanine-508 mediates a cytoplasmicmembrane domain contact in the CFTR 3D structure crucial to assembly and channel function. Proc Natl Acad Sci U S A 105:3256-3261.

85. Du, K., Sharma, M., and Lukacs, G.L. 2005. The DeltaF508 cystic fibrosis mutation impairs domain-domain interactions and arrests post-translational folding of CFTR. Nat Struct Mol Biol 12:17-25.

86. Rabeh, W.M., Bossard, F., Xu, H., Okiyoneda, T., Bagdany, M., Mulvihill, C.M., Du, K., di Bernardo, S., Liu, Y., Konermann, L., et al. 2012. Correction of both NBD1 energetics and domain interface is required to restore DeltaF508 CFTR folding and function. Cell 148:150-163.

87. Van Goor, F., Hadida, S., Grootenhuis, P.D., Burton, B., Cao, D., Neuberger, T., Turnbull, A., Singh, A., Joubran, J., Hazlewood, A., et al. 2009. Rescue of CF airway epithelial cell function in vitro by a CFTR potentiator, VX-770. Proc Natl Acad Sci U S A 106:18825-18830. 
88. Clancy, J.P., Rowe, S.M., Accurso, F.J., Aitken, M.L., Amin, R.S., Ashlock, M.A., Ballmann, M., Boyle, M.P., Bronsveld, I., Campbell, P.W., et al. 2012. Results of a phase IIa study of VX-809, an investigational CFTR corrector compound, in subjects with cystic fibrosis homozygous for the F508del-CFTR mutation. Thorax 67:12-18.

89. Stanton, B.A. 1997. Cystic fibrosis transmembrane conductance regulator (CFTR) and renal function. Wien Klin Wochenschr 109:457-464.

90. Gabriel, S.E., Brigman, K.N., Koller, B.H., Boucher, R.C., and Stutts, M.J. 1994. Cystic fibrosis heterozygote resistance to cholera toxin in the cystic fibrosis mouse model. Science 266:107-109.

91. Kunzelmann, K., and Mall, M. 2002. Electrolyte transport in the mammalian colon: mechanisms and implications for disease. Physiol Rev 82:245-289.

92. Romi, H., Cohen, I., Landau, D., Alkrinawi, S., Yerushalmi, B., Hershkovitz, R., Newman-Heiman, N., Cutting, G.R., Ofir, R., Sivan, S., et al. 2012. Meconium ileus caused by mutations in GUCY2C, encoding the CFTR-activating guanylate cyclase 2C. Am J Hum Genet 90:893-899.

93. Schulz, S., Green, C.K., Yuen, P.S., and Garbers, D.L. 1990. Guanylyl cyclase is a heat-stable enterotoxin receptor. Cell 63:941-948.

94. Vaandrager, A.B., Bot, A.G., Ruth, P., Pfeifer, A., Hofmann, F., and De Jonge, H.R. 2000. Differential role of cyclic GMP-dependent protein kinase II in ion transport in murine small intestine and colon. Gastroenterology 118:108-114.

95. Vaandrager, A.B., Smolenski, A., Tilly, B.C., Houtsmuller, A.B., Ehlert, E.M., Bot, A.G., Edixhoven, M., Boomaars, W.E., Lohmann, S.M., and de Jonge, H.R. 1998. Membrane targeting of cGMP-dependent protein kinase is required for cystic fibrosis transmembrane conductance regulator $\mathrm{Cl}$ - channel activation. Proc Natl Acad Sci U S A 95:1466-1471.

96. Resta-Lenert, S., and Barrett, K.E. 2002. Enteroinvasive bacteria alter barrier and transport properties of human intestinal epithelium: role of iNOS and COX-2. Gastroenterology 122:1070-1087.

97. Kosek, M., Bern, C., and Guerrant, R.L. 2003. The global burden of diarrhoeal disease, as estimated from studies published between 1992 and 2000. Bull World Health Organ 81:197-204.

98. Ren, A., Zhang, W., Thomas, H.G., Barish, A., Berry, S., Kiel, J.S., and Naren, A.P. 2012. A tannic acid-based medical food, Cesinex((R)), exhibits broadspectrum antidiarrheal properties: a mechanistic and clinical study. Dig Dis Sci 57:99-108. 
99. Xavier, R.J., and Podolsky, D.K. 2007. Unravelling the pathogenesis of inflammatory bowel disease. Nature 448:427-434.

100. Eckmann, L. 2006. Animal models of inflammatory bowel disease: lessons from enteric infections. Ann N Y Acad Sci 1072:28-38.

101. Goyette, P., Labbe, C., Trinh, T.T., Xavier, R.J., and Rioux, J.D. 2007. Molecular pathogenesis of inflammatory bowel disease: genotypes, phenotypes and personalized medicine. Ann Med 39:177-199.

102. Tang, Y., Forsyth, C.B., and Keshavarzian, A. 2011. New molecular insights into inflammatory bowel disease-induced diarrhea. Expert Rev Gastroenterol Hepatol 5:615-625.

103. Seidler, U., Lenzen, H., Cinar, A., Tessema, T., Bleich, A., and Riederer, B. 2006. Molecular mechanisms of disturbed electrolyte transport in intestinal inflammation. Ann N Y Acad Sci 1072:262-275.

104. Sandle, G.I. 2005. Pathogenesis of diarrhea in ulcerative colitis: new views on an old problem. J Clin Gastroenterol 39:S49-52.

105. Bresso, F., Askling, J., Astegiano, M., Demarchi, B., Sapone, N., Rizzetto, M., Gionchetti, P., Lammers, K.M., de Leone, A., Riegler, G., et al. 2007. Potential role for the common cystic fibrosis DeltaF508 mutation in Crohn's disease. Inflamm Bowel Dis 13:531-536.

106. Kleizen, B., Braakman, I., and de Jonge, H.R. 2000. Regulated trafficking of the CFTR chloride channel. Eur J Cell Biol 79:544-556.

107. Rachmilewitz, D., Eliakim, R., Ackerman, Z., and Karmeli, F. 1998. Direct determination of colonic nitric oxide level--a sensitive marker of disease activity in ulcerative colitis. Am J Gastroenterol 93:409-412.

108. Kimura, H., Hokari, R., Miura, S., Shigematsu, T., Hirokawa, M., Akiba, Y., Kurose, I., Higuchi, H., Fujimori, H., Tsuzuki, Y., et al. 1998. Increased expression of an inducible isoform of nitric oxide synthase and the formation of peroxynitrite in colonic mucosa of patients with active ulcerative colitis. Gut 42:180-187.

109. Guihot, G., Guimbaud, R., Bertrand, V., Narcy-Lambare, B., Couturier, D., Duee, P.H., Chaussade, S., and Blachier, F. 2000. Inducible nitric oxide synthase activity in colon biopsies from inflammatory areas: correlation with inflammation intensity in patients with ulcerative colitis but not with Crohn's disease. Amino Acids 18:229-237. 
110. Martin, M.C., Martinez, A., Mendoza, J.L., Taxonera, C., Diaz-Rubio, M., Fernandez-Arquero, M., de la Concha, E.G., and Urcelay, E. 2007. Influence of the inducible nitric oxide synthase gene (NOS2A) on inflammatory bowel disease susceptibility. Immunogenetics 59:833-837.

111. Zingarelli, B., Szabo, C., and Salzman, A.L. 1999. Reduced oxidative and nitrosative damage in murine experimental colitis in the absence of inducible nitric oxide synthase. Gut 45:199-209.

112. Beck, P.L., Xavier, R., Wong, J., Ezedi, I., Mashimo, H., Mizoguchi, A., Mizoguchi, E., Bhan, A.K., and Podolsky, D.K. 2004. Paradoxical roles of different nitric oxide synthase isoforms in colonic injury. Am J Physiol Gastrointest Liver Physiol 286:G137-147.

113. Banan, A., Zhang, L., Fields, J.Z., Farhadi, A., Talmage, D.A., and Keshavarzian, A. 2002. PKC-zeta prevents oxidant-induced iNOS upregulation and protects the microtubules and gut barrier integrity. Am J Physiol Gastrointest Liver Physiol 283:G909-922.

114. Schworer, H., Bohn, M., Waezsada, S.Y., Raddatz, D., and Ramadori, G. 2001. Successful treatment of megacolon associated with colitis with a nitric oxide synthase inhibitor. Am J Gastroenterol 96:2273-2274.

115. Jurjus, A.R., Khoury, N.N., and Reimund, J.M. 2004. Animal models of inflammatory bowel disease. J Pharmacol Toxicol Methods 50:81-92.

116. Perse, M., and Cerar, A. 2012. Dextran sodium sulphate colitis mouse model: traps and tricks. J Biomed Biotechnol 2012:718617.

117. Wirtz, S., Neufert, C., Weigmann, B., and Neurath, M.F. 2007. Chemically induced mouse models of intestinal inflammation. Nat Protoc 2:541-546.

118. Zaki, M.H., Boyd, K.L., Vogel, P., Kastan, M.B., Lamkanfi, M., and Kanneganti, T.D. 2010. The NLRP3 inflammasome protects against loss of epithelial integrity and mortality during experimental colitis. Immunity 32:379-391.

119. Lindsey, I., George, B.D., Kettlewell, M.G., and Mortensen, N.J. 2001. Impotence after mesorectal and close rectal dissection for inflammatory bowel disease. Dis Colon Rectum 44:831-835.

120. Fanning, A.S., and Anderson, J.M. 1999. PDZ domains: fundamental building blocks in the organization of protein complexes at the plasma membrane. J Clin Invest 103:767-772. 
121. Cha, B., and Donowitz, M. 2008. The epithelial brush border $\mathrm{Na}+/ \mathrm{H}+$ exchanger NHE3 associates with the actin cytoskeleton by binding to ezrin directly and via PDZ domain-containing $\mathrm{Na}^{+} / \mathrm{H}^{+}$exchanger regulatory factor (NHERF) proteins. Clin Exp Pharmacol Physiol 35:863-871.

122. Seidler, U., Singh, A.K., Cinar, A., Chen, M., Hillesheim, J., Hogema, B., and Riederer, B. 2009. The role of the NHERF family of PDZ scaffolding proteins in the regulation of salt and water transport. Ann N Y Acad Sci 1165:249-260.

123. Costello, C.M., Mah, N., Hasler, R., Rosenstiel, P., Waetzig, G.H., Hahn, A., Lu, T., Gurbuz, Y., Nikolaus, S., Albrecht, M., et al. 2005. Dissection of the inflammatory bowel disease transcriptome using genome-wide cDNA microarrays. PLoS Med 2:e199.

124. Mashanov, G.I., Tacon, D., Knight, A.E., Peckham, M., and Molloy, J.E. 2003. Visualizing single molecules inside living cells using total internal reflection fluorescence microscopy. Methods 29:142-152.

125. Soderberg, O., Gullberg, M., Jarvius, M., Ridderstrale, K., Leuchowius, K.J., Jarvius, J., Wester, K., Hydbring, P., Bahram, F., Larsson, L.G., et al. 2006. Direct observation of individual endogenous protein complexes in situ by proximity ligation. Nat Methods 3:995-1000.

126. Li, H., Vahlquist, A., and Torma, H. 2012. Interactions between FATP4 and ichthyin in epidermal lipid processing may provide clues to the pathogenesis of autosomal recessive congenital ichthyosis. J Dermatol Sci 69:195-201.

127. Whitaker, M. 2010. Genetically encoded probes for measurement of intracellular calcium. Methods Cell Biol 99:153-182.

128. Zaccolo, M., De Giorgi, F., Cho, C.Y., Feng, L., Knapp, T., Negulescu, P.A., Taylor, S.S., Tsien, R.Y., and Pozzan, T. 2000. A genetically encoded, fluorescent indicator for cyclic AMP in living cells. Nat Cell Biol 2:25-29.

129. Ponsioen, B., Zhao, J., Riedl, J., Zwartkruis, F., van der Krogt, G., Zaccolo, M., Moolenaar, W.H., Bos, J.L., and Jalink, K. 2004. Detecting cAMP-induced Epac activation by fluorescence resonance energy transfer: Epac as a novel cAMP indicator. EMBO Rep 5:1176-1180.

130. Honda, A., Adams, S.R., Sawyer, C.L., Lev-Ram, V., Tsien, R.Y., and Dostmann, W.R. 2001. Spatiotemporal dynamics of guanosine 3',5'-cyclic monophosphate revealed by a genetically encoded, fluorescent indicator. Proc Natl Acad Sci U S A 98:2437-2442. 
131. Kass, D.A., Takimoto, E., Nagayama, T., and Champion, H.C. 2007. Phosphodiesterase regulation of nitric oxide signaling. Cardiovasc Res 75:303314.

132. Tsai, E.J., and Kass, D.A. 2009. Cyclic GMP signaling in cardiovascular pathophysiology and therapeutics. Pharmacol Ther 122:216-238.

133. Galietta, L.V., Jayaraman, S., and Verkman, A.S. 2001. Cell-based assay for highthroughput quantitative screening of CFTR chloride transport agonists. Am J Physiol Cell Physiol 281:C1734-1742.

134. Goldenberg, M.M. 1998. Safety and efficacy of sildenafil citrate in the treatment of male erectile dysfunction. Clin Ther 20:1033-1048.

135. Kolios, G., Brown, Z., Robson, R.L., Robertson, D.A., and Westwick, J. 1995. Inducible nitric oxide synthase activity and expression in a human colonic epithelial cell line, HT-29. Br J Pharmacol 116:2866-2872.

136. Ostedgaard, L.S., Zabner, J., Vermeer, D.W., Rokhlina, T., Karp, P.H., Stecenko, A.A., Randak, C., and Welsh, M.J. 2002. CFTR with a partially deleted R domain corrects the cystic fibrosis chloride transport defect in human airway epithelia in vitro and in mouse nasal mucosa in vivo. Proc Natl Acad Sci U S A 99:30933098.

137. Ma, T., Thiagarajah, J.R., Yang, H., Sonawane, N.D., Folli, C., Galietta, L.J., and Verkman, A.S. 2002. Thiazolidinone CFTR inhibitor identified by highthroughput screening blocks cholera toxin-induced intestinal fluid secretion. $J$ Clin Invest 110:1651-1658.

138. Krieglstein, C.F., Cerwinka, W.H., Laroux, F.S., Salter, J.W., Russell, J.M., Schuermann, G., Grisham, M.B., Ross, C.R., and Granger, D.N. 2001. Regulation of murine intestinal inflammation by reactive metabolites of oxygen and nitrogen: divergent roles of superoxide and nitric oxide. J Exp Med 194:1207-1218.

139. McCafferty, D.M., Miampamba, M., Sihota, E., Sharkey, K.A., and Kubes, P. 1999. Role of inducible nitric oxide synthase in trinitrobenzene sulphonic acid induced colitis in mice. Gut 45:864-873.

140. MacNaughton, W.K. 1993. Nitric oxide-donating compounds stimulate electrolyte transport in the guinea pig intestine in vitro. Life Sci 53:585-593.

141. Seimetz, M., Parajuli, N., Pichl, A., Veit, F., Kwapiszewska, G., Weisel, F.C., Milger, K., Egemnazarov, B., Turowska, A., Fuchs, B., et al. 2011. Inducible NOS inhibition reverses tobacco-smoke-induced emphysema and pulmonary hypertension in mice. Cell 147:293-305. 
142. Wirtz, S., and Neurath, M.F. 2007. Mouse models of inflammatory bowel disease. Adv Drug Deliv Rev 59:1073-1083.

143. Boismenu, R., and Chen, Y. 2000. Insights from mouse models of colitis. $J$ Leukoc Biol 67:267-278.

144. Perreault, M., and Marette, A. 2001. Targeted disruption of inducible nitric oxide synthase protects against obesity-linked insulin resistance in muscle. Nat Med $7: 1138-1143$

145. Kroncke, K.D., Fehsel, K., and Kolb-Bachofen, V. 1998. Inducible nitric oxide synthase in human diseases. Clin Exp Immunol 113:147-156.

146. Seeliger, M.A., Breward, S.E., Friedler, A., Schon, O., and Itzhaki, L.S. 2003. Cooperative organization in a macromolecular complex. Nat Struct Biol 10:718724.

147. Navarro-Lerida, I., Corvi, M.M., Barrientos, A.A., Gavilanes, F., Berthiaume, L.G., and Rodriguez-Crespo, I. 2004. Palmitoylation of inducible nitric-oxide synthase at Cys-3 is required for proper intracellular traffic and nitric oxide synthesis. J Biol Chem 279:55682-55689.

148. Cuthbert, A.P., Fisher, S.A., Mirza, M.M., King, K., Hampe, J., Croucher, P.J., Mascheretti, S., Sanderson, J., Forbes, A., Mansfield, J., et al. 2002. The contribution of NOD2 gene mutations to the risk and site of disease in inflammatory bowel disease. Gastroenterology 122:867-874.

149. Hampe, J., Grebe, J., Nikolaus, S., Solberg, C., Croucher, P.J., Mascheretti, S., Jahnsen, J., Moum, B., Klump, B., Krawczak, M., et al. 2002. Association of NOD2 (CARD 15) genotype with clinical course of Crohn's disease: a cohort study. Lancet 359:1661-1665.

150. McGovern, D.P., Hysi, P., Ahmad, T., van Heel, D.A., Moffatt, M.F., Carey, A., Cookson, W.O., and Jewell, D.P. 2005. Association between a complex insertion/deletion polymorphism in NOD1 (CARD4) and susceptibility to inflammatory bowel disease. Hum Mol Genet 14:1245-1250.

151. Khor, B., Gardet, A., and Xavier, R.J. 2011. Genetics and pathogenesis of inflammatory bowel disease. Nature 474:307-317.

152. Peltekova, V.D., Wintle, R.F., Rubin, L.A., Amos, C.I., Huang, Q., Gu, X., Newman, B., Van Oene, M., Cescon, D., Greenberg, G., et al. 2004. Functional variants of OCTN cation transporter genes are associated with Crohn disease. Nat Genet 36:471-475. 
153. Schwab, M., Schaeffeler, E., Marx, C., Fromm, M.F., Kaskas, B., Metzler, J., Stange, E., Herfarth, H., Schoelmerich, J., Gregor, M., et al. 2003. Association between the C3435T MDR1 gene polymorphism and susceptibility for ulcerative colitis. Gastroenterology 124:26-33.

154. Stoll, M., Corneliussen, B., Costello, C.M., Waetzig, G.H., Mellgard, B., Koch, W.A., Rosenstiel, P., Albrecht, M., Croucher, P.J., Seegert, D., et al. 2004. Genetic variation in DLG5 is associated with inflammatory bowel disease. Nat Genet 36:476-480.

155. Rioux, J.D., Xavier, R.J., Taylor, K.D., Silverberg, M.S., Goyette, P., Huett, A., Green, T., Kuballa, P., Barmada, M.M., Datta, L.W., et al. 2007. Genome-wide association study identifies new susceptibility loci for Crohn disease and implicates autophagy in disease pathogenesis. Nat Genet 39:596-604.

156. Binder, H.J. 2009. Mechanisms of diarrhea in inflammatory bowel diseases. Ann N Y Acad Sci 1165:285-293.

157. Sullivan, S., Alex, P., Dassopoulos, T., Zachos, N.C., Iacobuzio-Donahue, C., Donowitz, M., Brant, S.R., Cuffari, C., Harris, M.L., Datta, L.W., et al. 2009. Downregulation of sodium transporters and NHERF proteins in IBD patients and mouse colitis models: potential contributors to IBD-associated diarrhea. Inflamm Bowel Dis 15:261-274.

158. Dykhuizen, R.S., Masson, J., McKnight, G., Mowat, A.N., Smith, C.C., Smith, L.M., and Benjamin, N. 1996. Plasma nitrate concentration in infective gastroenteritis and inflammatory bowel disease. Gut 39:393-395.

159. Donowitz, M., Mohan, S., Zhu, C.X., Chen, T.E., Lin, R., Cha, B., Zachos, N.C., Murtazina, R., Sarker, R., and Li, X. 2009. NHE3 regulatory complexes. J Exp Biol 212:1638-1646.

160. Donowitz, M., and Li, X. 2007. Regulatory binding partners and complexes of NHE3. Physiol Rev 87:825-872.

161. Hoogerwerf, W.A., Tsao, S.C., Devuyst, O., Levine, S.A., Yun, C.H., Yip, J.W., Cohen, M.E., Wilson, P.D., Lazenby, A.J., Tse, C.M., et al. 1996. NHE2 and NHE3 are human and rabbit intestinal brush-border proteins. Am J Physiol 270:G29-41.

162. Zaman, K., Carraro, S., Doherty, J., Henderson, E.M., Lendermon, E., Liu, L., Verghese, G., Zigler, M., Ross, M., Park, E., et al. 2006. S-nitrosylating agents: a novel class of compounds that increase cystic fibrosis transmembrane conductance regulator expression and maturation in epithelial cells. Mol Pharmacol 70:1435-1442. 
163. Chen, L., Patel, R.P., Teng, X., Bosworth, C.A., Lancaster, J.R., Jr., and Matalon, S. 2006. Mechanisms of cystic fibrosis transmembrane conductance regulator activation by S-nitrosoglutathione. J Biol Chem 281:9190-9199.

164. Zhang, W., Penmatsa, H., Ren, A., Punchihewa, C., Lemoff, A., Yan, B., Fujii, N., and Naren, A.P. 2011. Functional regulation of cystic fibrosis transmembrane conductance regulator-containing macromolecular complexes: a small-molecule inhibitor approach. Biochem J 435:451-462.

165. Valentine, C.D., Lukacs, G.L., Verkman, A.S., and Haggie, P.M. 2012. Reduced PDZ Interactions of Rescued DeltaF508CFTR Increases Its Cell Surface Mobility. $J$ Biol Chem 287:43630-43638.

166. Wu, Y., Wang, S., Farooq, S.M., Castelvetere, M.P., Hou, Y., Gao, J.L., Navarro, J.V., Oupicky, D., Sun, F., and Li, C. 2012. A chemokine receptor CXCR2 macromolecular complex regulates neutrophil functions in inflammatory diseases. J Biol Chem 287:5744-5755. 


\section{VITA}

Kavisha Arora was born in Delhi, India in the year 1985. She obtained Bachelor of Technology in Biotechnology from GGS Indraprastha University in New Delhi, India in May 2009. She was enrolled in Integrated Program in Biomedical Science (IPBS) for doctoral studies at University of Tennessee Health Science Center, Memphis, TN, USA in August 2009. She received her doctorate degree in August 2013. 